\title{
Structural characterization of autophagy related protein complexes
}

\author{
Dissertation \\ for the award of the degree \\ "Doctor rerum naturalium" \\ of the Georg-August-University Göttingen
}

within the doctoral program "Biology"

of the Georg-August University School of Science (GAUSS)

submitted by

Janina Metje

from Gehrden

Göttingen, 2017 


\section{Supervisor}

Dr. Karin Kühnel

Research Group Autophagy, Department of Neurobiology

Max Planck Institute for Biophysical Chemistry, Göttingen

Present address: Nature Communications, London

Member of the Thesis Committee (First Reviewer):

Prof. Dr. Michael Thumm

Institute of Cellular Biochemistry, Center for Biochemistry and Molecular Cell Biology Georg-August-University, Göttingen

\section{Member of the Thesis Committee (Second Reviewer):}

Prof. Dr. Heike Krebber

Institute for Microbiology and Genetics, Department of Molecular Genetics

Georg-August-University, Göttingen

\section{Members of the extended Thesis Committee}

Prof. Dr. Ivo Feußner

Department for Plant Biochemistry, Albrecht-von-Haller-Institute for Plant Sciences

Georg-August-University, Göttingen

Prof. Dr. Blanche Schwappach

Department of Molecular Biochemistry, Center for Biochemistry and Molecular Cell Biology Georg-August-University, Göttingen

Dr. Vladimir Pena

Research Group Macromolecular Crystallography, Department of Cellular Biochemistry Max Planck Institute for Biophysical Chemistry, Göttingen

Prof. Dr. Henning Urlaub

Research Group Bioanalytical Mass Spectrometry, Department of Cellular Biochemistry Max Planck Institute for Biophysical Chemistry, Göttingen

Date of the oral examination: 22.05 .2017 


\section{Declaration of Authorship}

Hereby, I declare that I prepared the thesis "Structural characterization of autophagy related protein complexes" on my own and with no other sources and materials than cited.

Göttingen, 04.04.2017

Janina Metje 



\section{Abstract}

Autophagy is a conserved and highly regulated process in eukaryotic cells that plays an important role in maintaining cellular homeostasis. During macroautophagy a double membrane grows de novo that encloses cytoplasmic cargo and after its fusion an autophagosome vesicle is formed. The autophagosome then fuses with the vacuole or lysosome, where its content is degraded. The Atg12 Atg5/Atg16 complex is an essential part of the core autophagy machinery and localizes to the outside of the growing isolation membrane. The complex also acts as an E3-like ligase for the lipidation of ubiquitin-like Atg8. The PROPPIN ( $\beta$-propeller that binds polyphosphoinositides) Atg21 determines the site of Atg8 lipidation in yeast by interacting with both the coiled coil domain of Atg16 and Atg8.

In my first project, I obtained first low resolution insights into the interactions of Atg21 with the coiled coil domain of Atg16. The $4.0 \AA$ crystal structure shows that the Ashbya gossypii Atg16 coiled coil domain is at the center of the Atg21-Atg16 complex and interacts with two Kluyveromyces lactis Atg21 molecules on either site of the C-terminal ends of the coiled coil dimer. The two Atg21 molecules adopt a reversed V shape and their PI(3)P binding sites are located opposite to the Atg16 binding site. The structure thus shows how membrane bound Atg21 can bind an Atg16 dimer. However, at $4.0 \AA$ resolution molecular details of complex interaction are not visible. I also determined crystal structure of the coiled coil domain of AgAtg16 comprising residues 43-108 at 3.4 A resolution. Analysis of Atg21-Atg16 complex formation by analytic gel filtration revealed the importance of residues K1Atg21 R103E and AgAtg16 (70-124) D78R for binding. The structure of Atg21-Atg16 complex gives more insights into the coordination of Atg8 lipidation.

The coiled coil domain of mammalian Atg16 is an effector of Golgi-resident Rab33B. In my second project, I determined the crystal structure of murine Rab33B with the Atg16L1 coiled coil domain at $3.47 \AA$ resolution. The structure revealed that two Rab33B molecules form a complex with the diverging C-termini of one Atg16L1 dimer. Protein-protein interactions observed in the structure were confirmed by cross linking of the Rab33B(30-202)Q92LAtg16L1(153-210) complex and analysis by mass spectrometry. Based on the structure Rab33B and Atg16L1 mutants were designed to verify the Rab33B-Atg16L1 interactions. Both in vivo and in vitro pull-down experiments showed that selected single point mutations 
disrupted complex formation. Furthermore, immunofluorescence studies showed that these mutations abolished co-localization of Rab33B and Atg16L1 in cells. The Rab33B binding site of Atg16 identified in this study comprises residues 191-208 and is in close proximity of the PROPPIN WIPI2B binding site (207-230) and could explain how Golgi-derived vesicles can be recruited into close proximity of the isolation membrane by binding of Atg16 to both Rab33B and WIPI2B, providing a source of lipids to the growing isolation membrane.

In my third project, I characterized the SCOC-FEZ1 complex that has a regulatory role in autophagy. Complex formation is mediated through the dimeric coiled coil domains of both proteins. Crystals diffracting up to $2.2 \AA$ resolution were obtained but due a twinning problem the structure could not be determined. However, I gained new insights into SCOC-FEZ1 complex formation through biophysical experiments. I showed that the two dimers interact with a 1:1 stoichiometry with SEC-MALLS experiments. Cross-linking and analysis by mass spectroscopy revealed that FEZ1 most likely a forms parallel coiled coil dimer and that the SCOC and FEZ1 dimers interact in a parallel orientation with each other. 


\section{Table of contents}

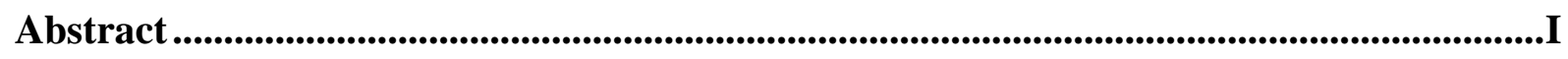

Table of contents..........................................................................................................................III

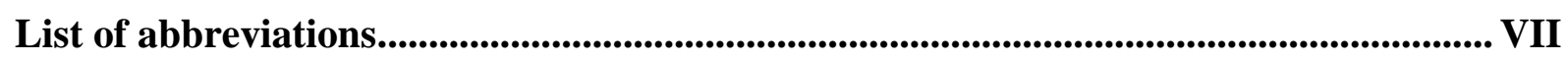

List of figures ......................................................................................................................................IX

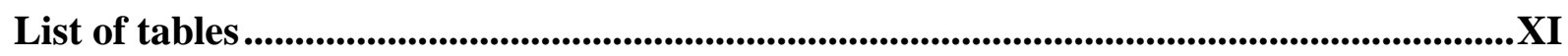

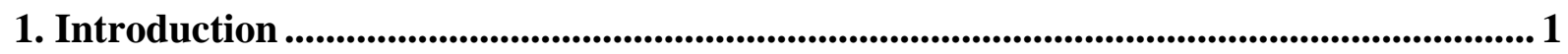

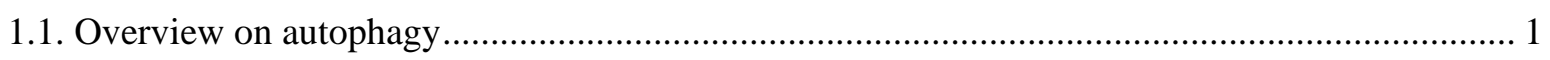

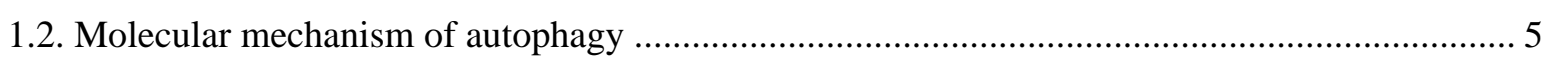

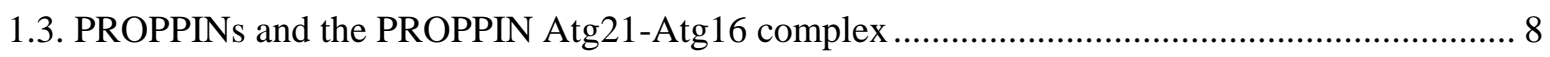

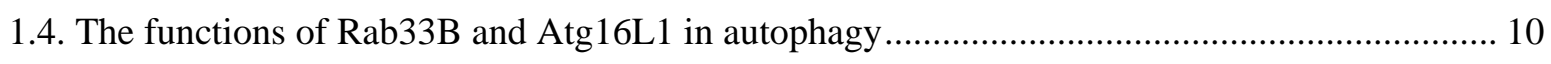

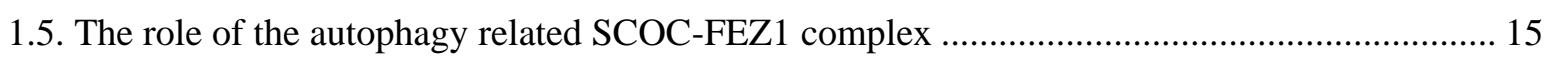

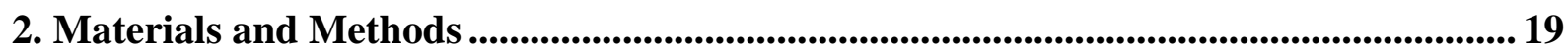

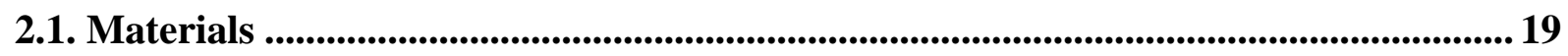

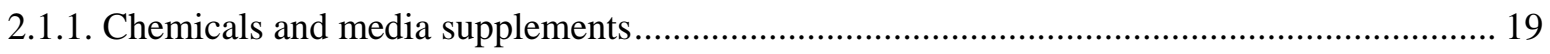

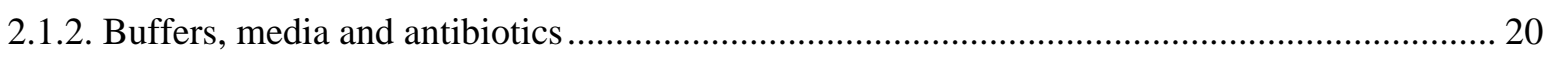

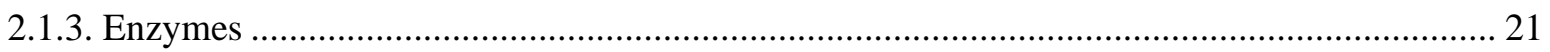

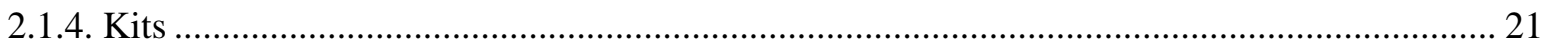

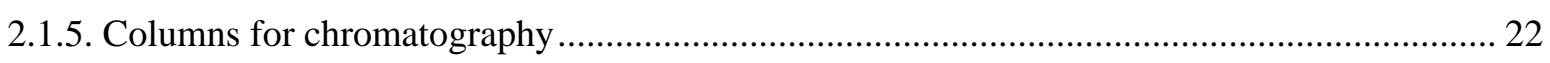

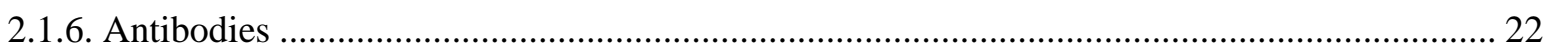

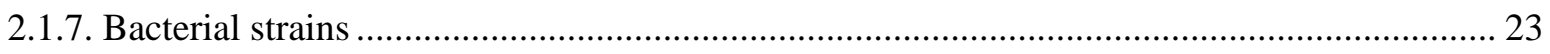

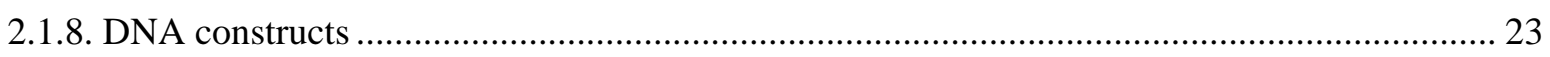

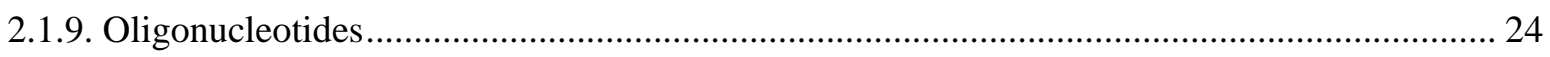

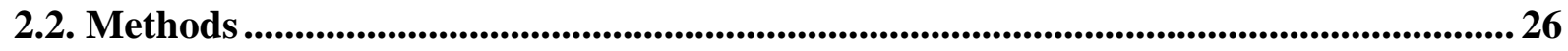

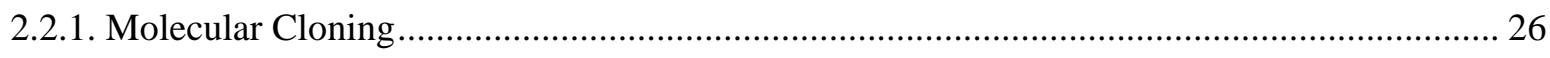

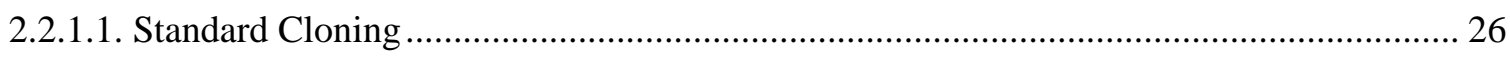

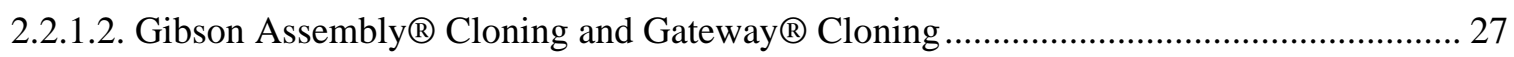

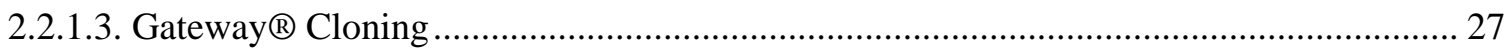

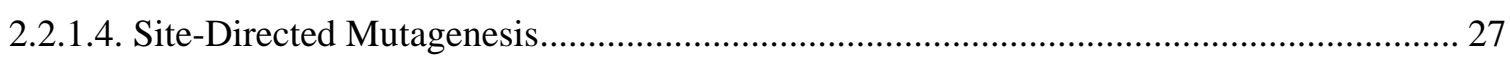

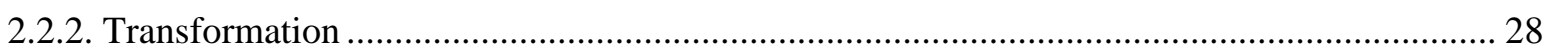

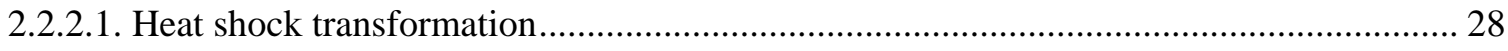

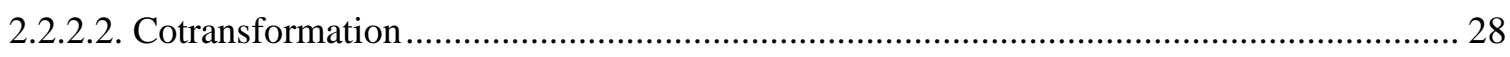

2.2.2.3. Transformation for GIBSON Assembly and mutagenesis ............................................ 28

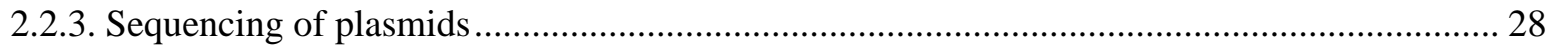

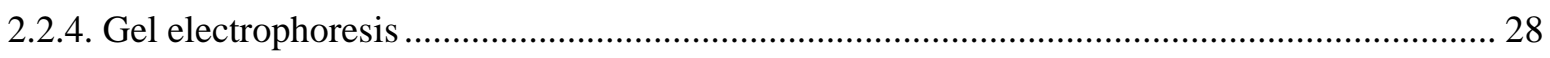

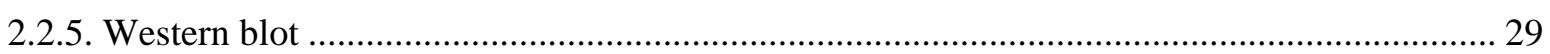

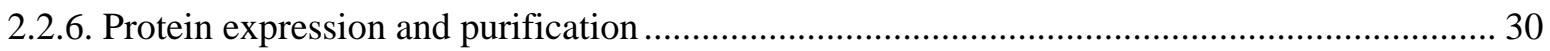

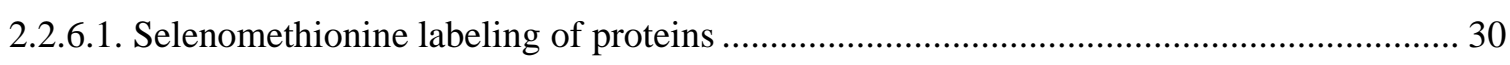

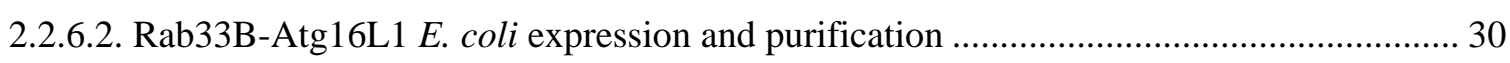


2.2.6.3. Rab33B-Atg16L1 HEK 293T cell expression and transfection ...................................... 31

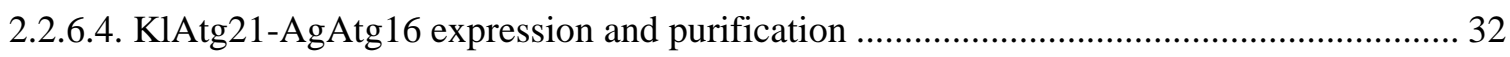

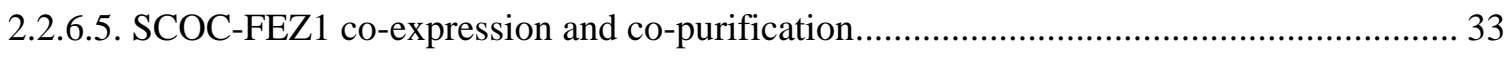

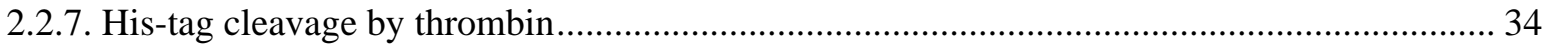

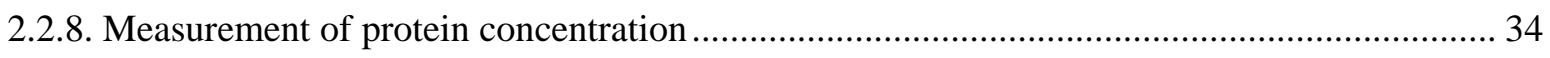

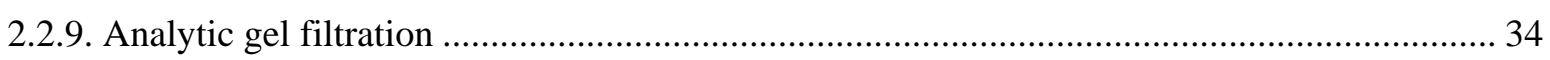

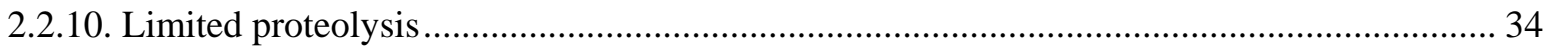

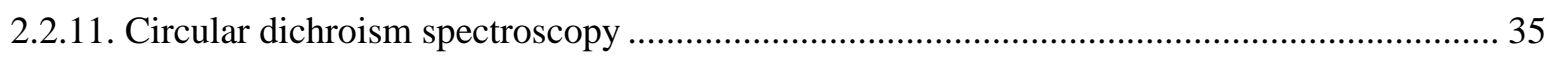

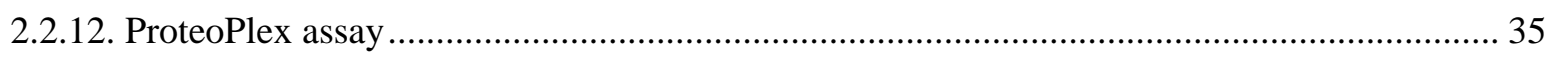

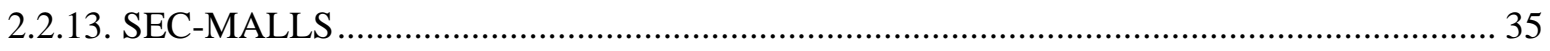

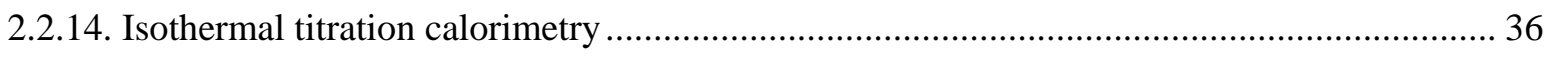

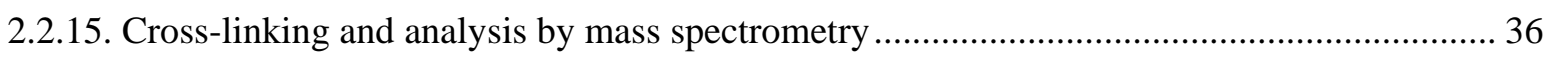

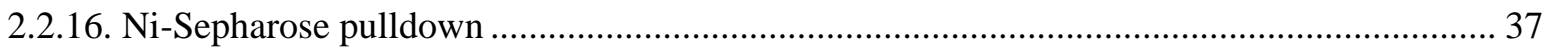

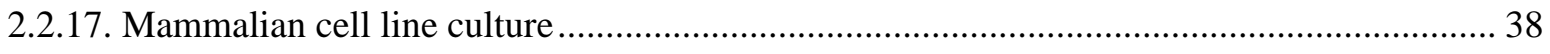

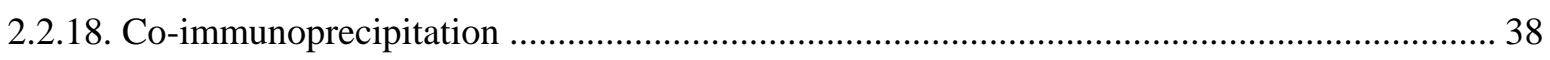

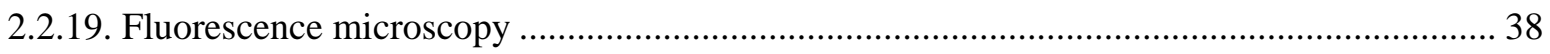

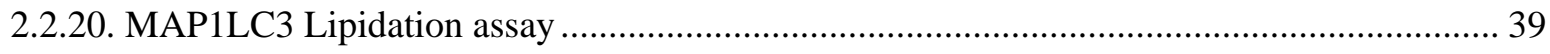

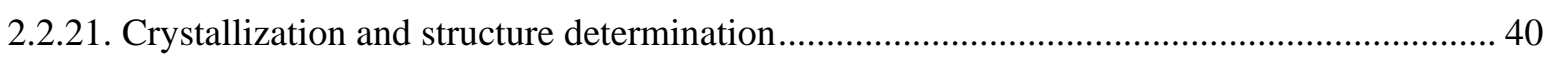

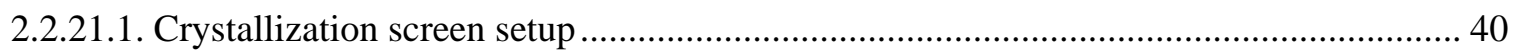

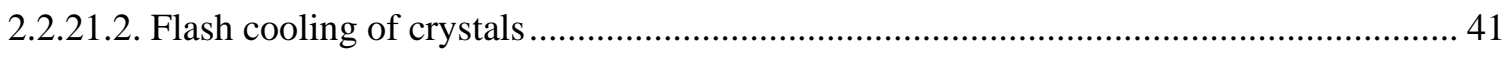

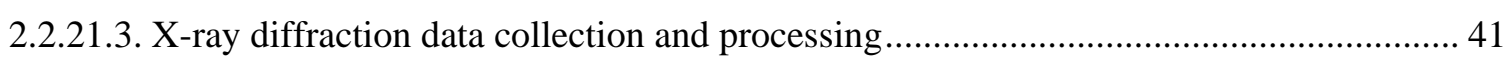

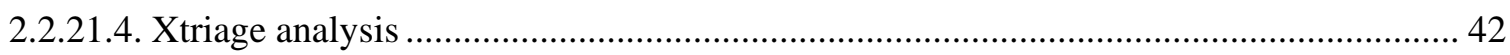

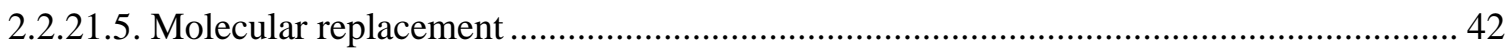

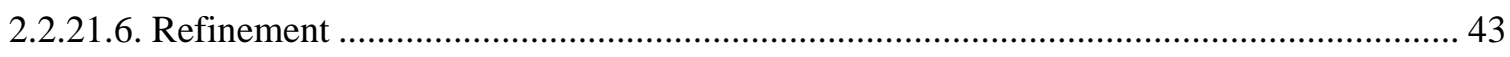

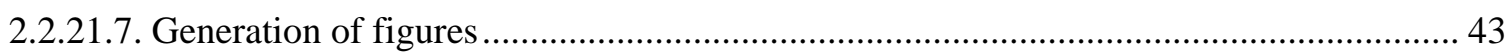

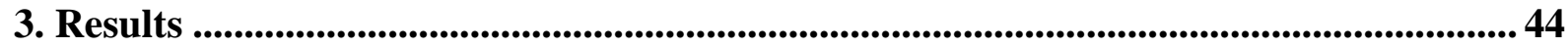

3.1. Structural characterization of the murine Rab33B-Atg16L1 complex....................... 44

3.1.1 Recombinant expression and purification of Rab33B, Atg16L1 and Rab33B-Atg16L1

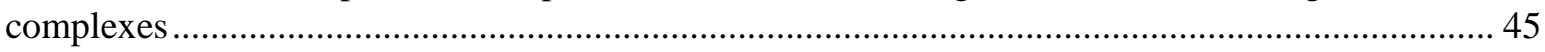

3.1.2. Biochemical and biophysical characterization of Rab33B-Atg16L1 complex formation........ 47

3.1.2.1. ProteoPlex assay for the Rab33B-Atg16L1 complex ....................................................... 47

3.1.2.2. Limited proteolysis of the Rab33B-Atg16L1 complex ................................................... 48

3.1.2.3. Isothermal titration calorimetry measurements of the Rab33B-Atg16L1 complex ......... 50

3.1.3. Structure determination of the Rab33B(30-202)Q92L-Atg16L1(153-210) complex ............. 51

3.1.3.1. Crystallization of the Rab33B-Atg16L1 complex ....................................................... 51

3.1.3.2. X-ray data collection and processing for the Rab33B(30-202)Q92L-Atg16L1(153-210)

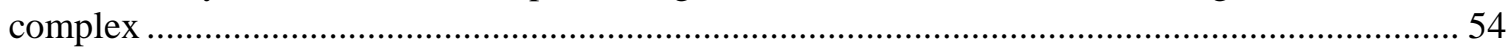

3.1.3.3. Matthew's coefficient for the Rab33B-Atg16L1 complex ............................................... 55

3.1.3.4. Molecular replacement for the Rab33B-mAtg16L1 complex ....................................... 55

3.1.3.5. Refinement and structure validation for the Rab33B-Atg16L1 complex......................... 57

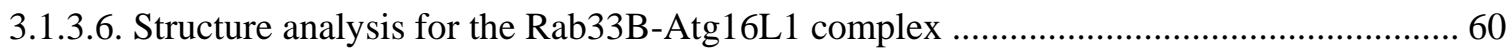


3.1.4. Cross-linking of the Rab33B-Atg $16 \mathrm{~L} 1$ complex and analysis by mass spectrometry ............ 68

3.1.5. Analyzing complex formation of Rab33B and Atg16L1 mutants in vitro .............................. 74

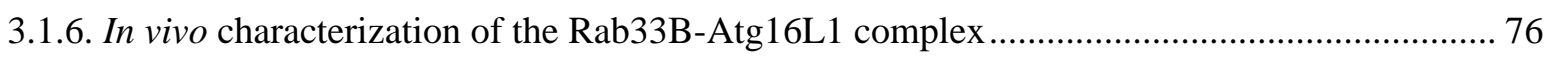

3.1.6.1. Expression of full length Rab33B and Atg16L1 in HEK293 cells.................................... 76

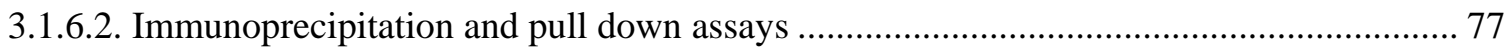

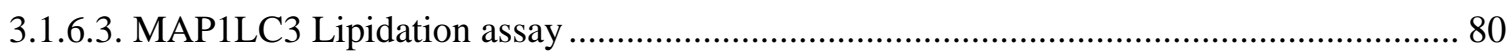

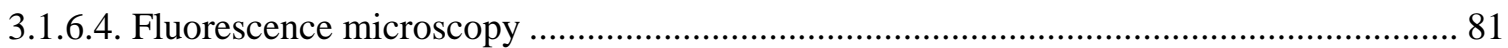

3.2. Characterization of the KIAtg21-AgAtg16 complex ......................................................... 85

3.2.1. Expression and purification of KlAtg21, AgAtg16 and KlAtg21-AgAtg16 complexes......... 85

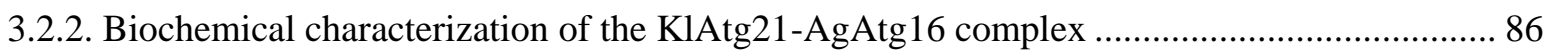

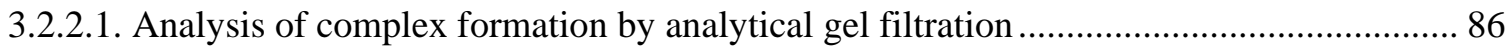

3.2.2.2. Analysis of the KlAtg21-AgAtg16 complex stoichiometry by SEC-MALLS

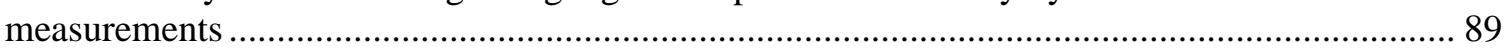

3.2.2.3. Isothermal calorimetry measurements of the KlAtg21-AgAtg 16 complex...................... 91

3.2.3. Structure determination of the KlAtg21-AgAtg 16 complex ................................................. 92

3.2.3.1. X-ray data collection and processing for the KlAtg21-AgAtg16 complex ....................... 92

3.2.3.2. Molecular replacement for the KlAtg21-AgAtg16 complex........................................... 93

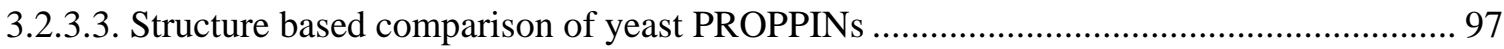

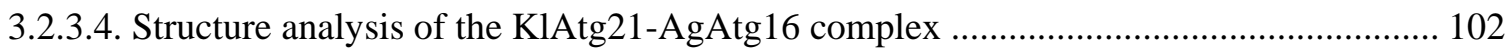

3.2.4. Identification of the KlAtg21 - AgAtg16 binding site by charge modification and analytic gel

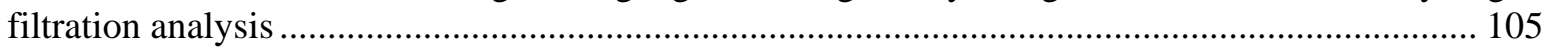

3.2.5. Hypothetical model for the coordination of Atg21 and the Atg12 Atg5/Atg16 complex .... 108

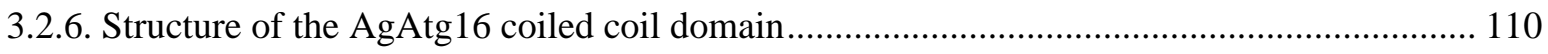

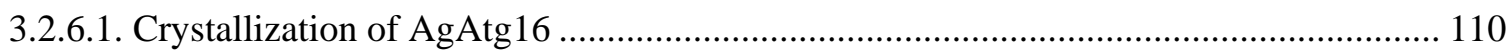

3.2.6.2. Mutagenesis of AgAtg16 for selenomethionine labeling ........................................... 112

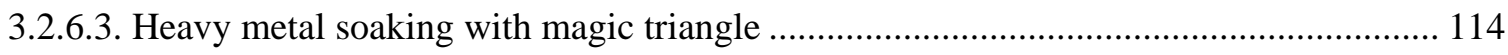

3.2.6.4. X-ray data collection and processing for AgAtg16 ..................................................... 114

3.2.6.5. Matthews coefficient for the AgAtg16(40-124) crystal ................................................ 115

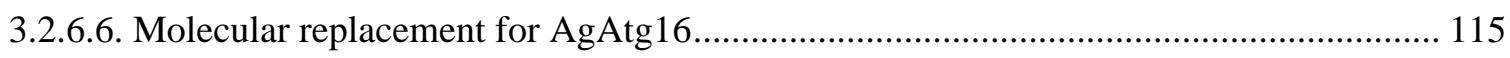

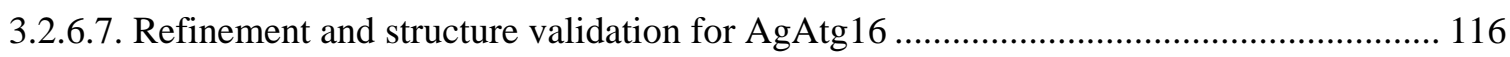

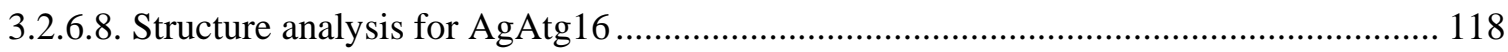

3.3. Characterization of the SCOC-FEZ1 complex....................................................... 122

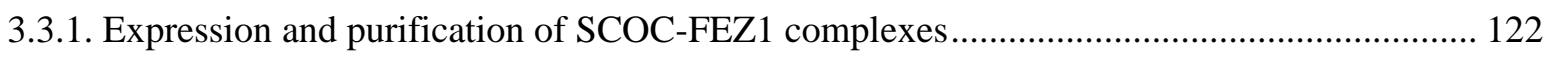

3.3.2. Biochemical characterization of the SCOC (78-159)-FEZ1 (225-295) complex................... 125

3.3.2.1. Analysis of secondary structure and stability of the SCOC-FEZ1 complex by CD

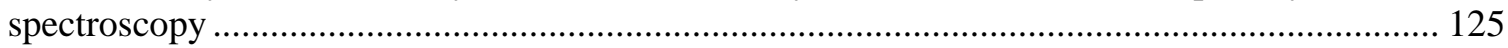

3.3.2.2. Stoichiometric analysis of the SCOC(78-159)-FEZ1 (225-295) complex by SEC-MALLS

.

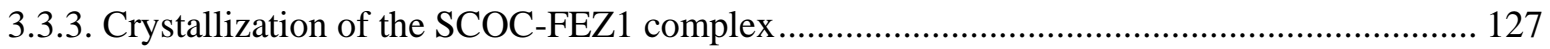

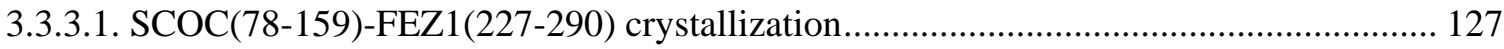

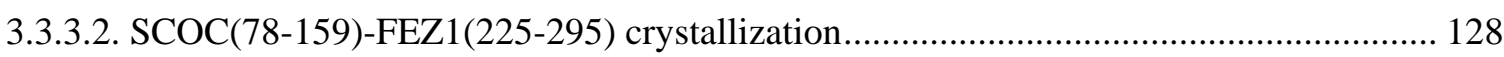


3.3.3.3. Optimization of SCOC(78-159)-FEZ1(225-295) crystals in PEG 20000 conditions..... 130 3.3.3.4. X-ray data collection and analysis of SCOC(78-159)-FEZ1(225-295) crystals from PEG 20.000 conditions

3.3.3.5. Optimization of SCOC(78-159)-FEZ1(225-295) crystals in isopropanol conditions .... 139

3.3.3.6. X-ray data collection and analysis of SCOC(78-159)-FEZ1(225-295) crystals from

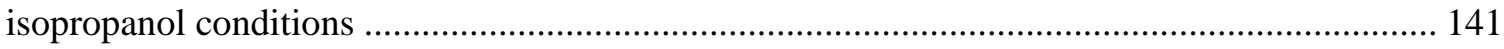

3.3.4. Cross-linking of the SCOC-FEZ1 complex and analysis by mass spectrometry .................. 144

4. Discussion ............................................................................................................................... 151

4.1. Two Rab33B molecules form a complex with the diverging C-termini of an Atg16L1

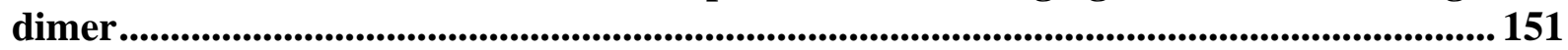

4.2. The Atg16 dimer forms the center of the Atg21-Atg16 complex ................................ 157

4.3. Insights into SCOC-FEZ1 complex formation .......................................................... 161

Bibliography .................................................................................................................................. 163

Acknowledgments.................................................................................................................... 171

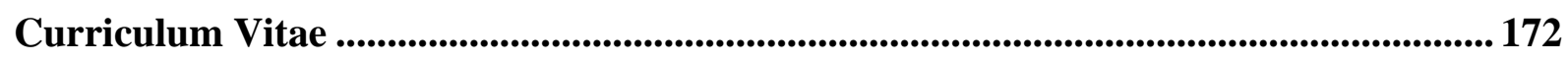




\section{List of abbreviations}

aa

Ag

Ams1

APS

Atg

Atg16L

AU

bp

BS3

C

ccd

CD

CL

CMA

CV

cvt

$\mathrm{Da}$

DNA

DMEM

DMEM/F-12

ECL

E. coli

EDTA

EG

ER

FBS

FEZ1

$\mathrm{ft}$

fwd

GAP

GDP

GEF

GFP

GppNHp

GTP

GTP $\gamma \mathrm{S}$

His

HRP

hsc70

Hsv2

IPTG

ITC

$\mathrm{kb}$

$\mathrm{Kl}$

LAMP-2A

LB

LC-MS/MS

LLG

$\mathrm{m}$

MAD

MALLS

MAP1LC3

ML

MR

Amino acid

Ashbya gossypii

$\alpha$-mannosidase

Ammonium persulfate

Autophagy-related

Autophagy-like

Asymmetric unit

Base pair

Bis(sulfosuccinimidyl) suberate

Celsius

Coiled coil domain

Circular dichroism

Clostripain

Chaperone-mediated autophagy

Column volume

Cytoplasm-to vacuole targeting

Dalton

Deoxyribonucleic acid

Dulbecco's Modified Eagle Medium

Dulbecco's Modified Eagle Medium/Nutrient Mixture F-12

Enhanced chemiluminescence

Escherichia coli

Ethylenediaminetetraacetic acid

Ethylene glycol

Endoplasmic reticulum

Fetal bovine serum

Fasciculation and elongation protein zeta 1

Flow-through

Forward

GTPase-activating protein

Guanosine diphosphate

Guanine nucleotide exchange factor

Green fluorescent protein

Guanosine-5'-[( $\beta, \gamma)$-imido]triphosphate

Guanosine triphosphate

Guanosine 5'-O-[ $\gamma$-thio]triphosphate

Hexahistidine

Horseradish peroxidase

Heat shock cognate protein of $70 \mathrm{kDa}$

Homologous with swollen vacuole phenotype 2

Isopropyl $\beta$-D-1-thiogalactopyranoside

Isothermal titration calorimetry

Kilo base

Kluyveromyces lactis

Lysosome-associated membrane protein type $2 \mathrm{~A}$

Luria Bertani

Liquid chromatography-tandem mass spectrometry

Log-likelihood gain

Murine

Multi wavelength anomalous diffraction or dispersion

Multi Angle Laser Light Scatterer

Microtubule-associated proteins 1A/1B light chain 3B

Mother liquor

Molecular replacement 


\begin{tabular}{|c|c|}
\hline MSC & Multiple cloning site \\
\hline MWCO & Molecular weight cut off \\
\hline $\mathrm{Ni}$ & Nickel $\left(\mathrm{Ni}^{2+}\right)$ \\
\hline NTA & Nitrilotriacetic acid \\
\hline NV & Nucleus-vacuole \\
\hline OD600 & Optical density at $600 \mathrm{~nm}$ \\
\hline $\mathrm{o} / \mathrm{n}$ & Over night \\
\hline $\mathrm{Pa}$ & Pichia angusta \\
\hline PAS & Preautophagosomal assembly site \\
\hline PBS & Phosphate buffered saline \\
\hline PCR & Polymerase chain reaction \\
\hline PDB & Protein Data Base \\
\hline PE & Phosphatidylethanolamine \\
\hline PEG & Poly ethylene glycol \\
\hline PI & Phosphoinositide \\
\hline $\mathrm{PI}(3) \mathrm{P}$ & Phosphatidylinositol 3-phosphate \\
\hline $\mathrm{PI}(3,5) \mathrm{P}_{2}$ & Phosphatidylinositol 3,5-bisphosphate \\
\hline PMN & Piecemeal microautophagy of the nucleus \\
\hline preApe1 & Precursor form of aminopeptidase I \\
\hline PROPPIN & $\beta$-propeller proteins that bind polyphosphoinositides \\
\hline Rab & Ras-related in brain \\
\hline rev & Reverse \\
\hline RMSD & Root-mean-square deviation of atomic positions \\
\hline $\mathrm{rpm}$ & Rounds per minute \\
\hline RT & Room temperature, $22-25^{\circ} \mathrm{C}$ \\
\hline SAD & Single wavelength anomalous diffraction or dispersion \\
\hline Sc & Saccharomyces cerevisiae \\
\hline $\mathrm{SCOC}$ & Short coiled coil protein \\
\hline SDS-PAGE & Sodium dodecyl sulfate polyacrylamide gel electrophoresis \\
\hline SEC & Size Exclusion Chromatography \\
\hline SOC & Super optimal broth with catabolite repression \\
\hline SNAREs & Soluble N-ethylmaleimide-sensitive fusion attachment protein receptors \\
\hline TAE & Tris-acetate-EDTA \\
\hline TB & Terrific broth \\
\hline TCEP & Tris(2-carboxyethyl)phosphine hydrochloride \\
\hline TFZ & Rotation function Z-score \\
\hline TOR & Target of rapamycin \\
\hline $\mathrm{U}$ & Unit \\
\hline UVRAG & UV radiation resistance associated gene \\
\hline WIPI & WD-40 repeat containing protein that interacts with PIs \\
\hline WT & Wild-type \\
\hline$(\mathrm{w} / \mathrm{v})$ & Weight per volume \\
\hline$(\mathrm{v} / \mathrm{v})$ & Volume per volume \\
\hline
\end{tabular}




\section{List of figures}

Figure 1.1.1.: Schematic overview on macroautophagy ...................................................................... 1

Figure 1.1.2.: Schematic overview of autophagy subtypes and their morphology ................................ 3

Figure 1.2.1.: Schematic overview of the molecular mechanisms of autophagy ................................... 6

Figure 1.2.2.: Crystal structures of the ATG12 ATG5/ATG16N complex and the Atg 16 coiled coil

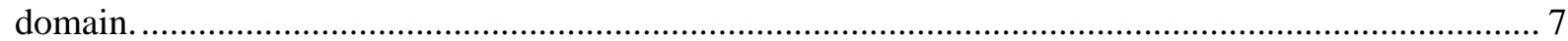

Figure 1.3.1.: Structure of the PROPPIN Kluyveromyces lactis Hsv2 ............................................. 9

Figure 1.3.2.: Model for the coordination of Atg8 lipidation....................................................... 10

Figure 1.4.1.: Schematic overview of Rab GTPases involvement in autophagy .................................. 10

Figure 1.4.2.: Rab33B structures in the active and inactive state.................................................. 12

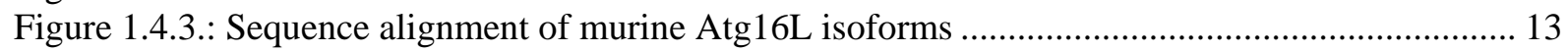

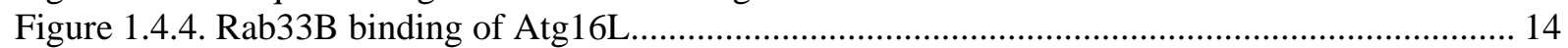

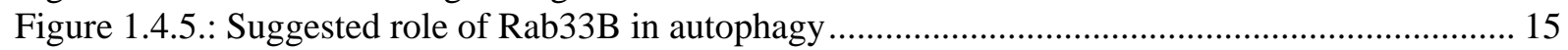

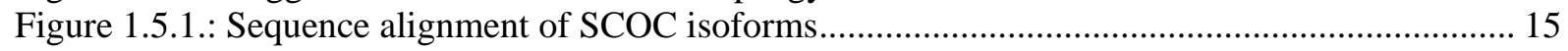

Figure 1.5.2.: Model for the possible SCOC-FEZ1 complex involvement in autophagy ..................... 17

Figure 1.5.3.: Crystal structure of the SCOC coiled coil domain..................................................... 18

Figure 1.5.4.: Domaine structure of human SCOC and FEZ1 ...................................................... 18

Figure 3.1.1.: Various truncated mAtg16L1 ccd constructs tested for Rab33B (30-202)Q92L binding.

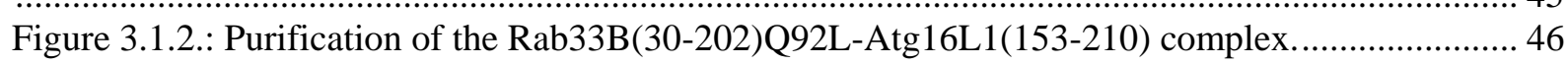

Figure 3.1.3.: Purification of the Rab33B(30-202)Q92L-Atg16L1(163-210) complex.......................... 47

Figure 3.1.4.: ProteoPlex unfolding curves of the Rab33B(30-202)Q92L-Atg16L1(153-210) complex

Figure 3.1.5.: Comparison of Rab33B(30-202)Q92L-Atg16L1(153-210) complex purification with

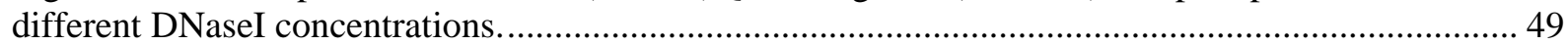

Figure 3.1.6.: Limited proteolysis of the Rab33B-Atg16L1 complex.............................................. 50

Figure 3.1.7.: Isothermal titration calorimetry titration curves of mRab33B(30-202)Q92L and mAtg16L1(153-210) ….................................................................................................... 51

Figure 3.1.8.: Initial needle like crystals from Rab33B(30-202)Q92L-Atg16L1(153-210)................. 52

Figure 3.1.9.: Rab33B(30-202)Q92L-Atg16L1(153-210) crystals using the Hampton Additive Screen

Figure 3.1.10.: Rab33B(30-202)Q92L-Atg16L1(153-210) crystal mounted at the Swiss Light Source

Figure 3.1.11.: Sequence alignment of yeast and mouse Atg16 ........................................... 56

Figure 3.1.12.: Refinement statistic plots for the Rab33B(30-202)Q92L-Atg16L1(153-210) structure

Figure 3.1.13.: Three Rab33B(30-202)Q92L-Atg16L1(153-210) complexes are present in the

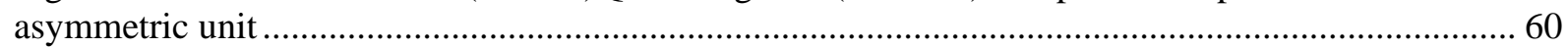

Figure 3.1.14.: Structure of the Rab33B(30-202)Q92L-Atg16L1(153-210) complex .......................... 61

Figure 3.1.15.: Crystal packing of the Rab33B(30-202)Q92L-Atg16L1(153-210) crystal.................. 62

Figure 3.1.16.: Alignment of the three Rab33B(30-202)Q92L-Atg16L1(153-210) complexes found in

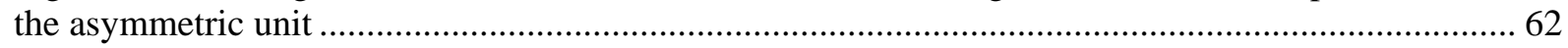

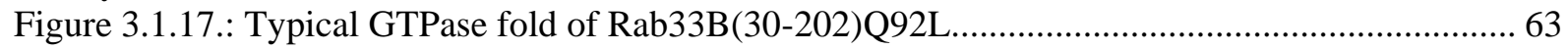

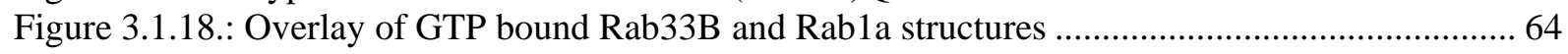

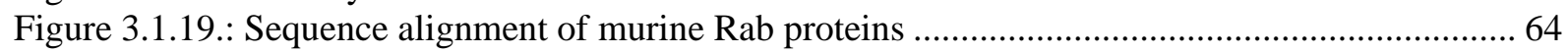

Figure 3.1.20.: Overlay of Rab33B structures in different nucleotide bound states ............................. 65

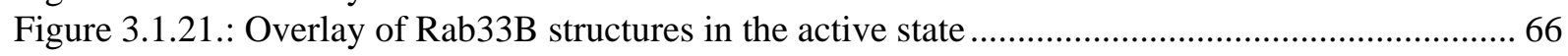

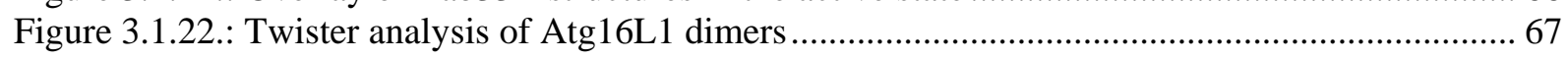

Figure 3.1.23.: Overlay of the Atg16L1 structure and an ideal Atg16L1 model ................................. 67

Figure 3.1.24.: Helical wheel projection of the residues in the Atg16L1 dimer IJ ..............................6 68

Figure 3.1.25.: Cross-linking of the Rab33B-Atg16L1 complex .................................................... 69

Figure 3.1.26.: Visualization of the mass spectrometry analysis of the cross-linking of the Rab33B-

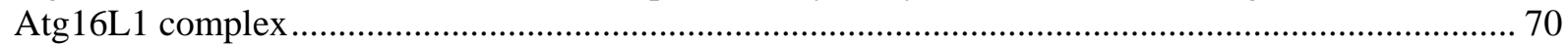

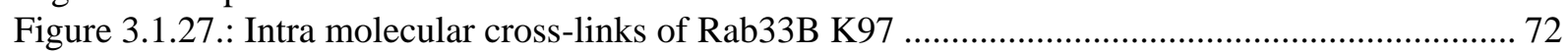

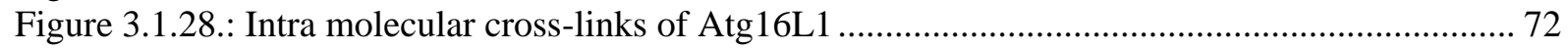


Figure 3.1.29.: Cross-links between Rab33B and Atg16L1 ............................................................. 73

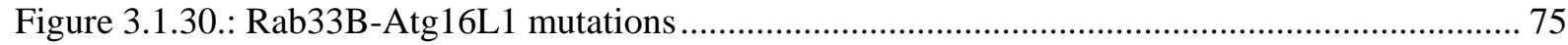

Figure 3.1.31.: Ni-sepharose pulldown of Rab33B(30-202)Q92L and Atg16L1(153-210) K198A.... 75

Figure 3.1.32.: Ni-Sepharose pulldown of Rab33B(30-202) and Atg16L1(153-210) ........................ 76

Figure 3.1.33.: Western blot analysis to characterize the overexpression of Atg16L1-EmGFP and V5-

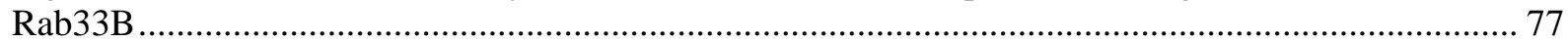

Figure 3.1.34.: GFP co-immunoprecipitation of Atg16L1-EmGFP and V5-Rab33B variants ............... 79

Figure 3.1.35.: V5 co-immunoprecipitation of Atg16L1-EmGFP and V5-Rab33B variants............... 80

Figure 3.1.37.: Intracellular distribution of EmGFP-Atg16L1 and V5-Rab33B WT/ Q92L or T47N . 82 Figure 3.1.38.: Intracellular distribution of EmGFP-Atg16L1 and V5-Rab33B Q92L F70A/ F70E or

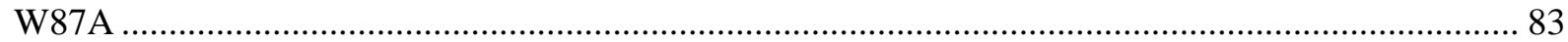

Figure 3.1.39.: Intracellular distribution of EmGFP-Atg16L1 K198A/ A202W or N206K and V5-

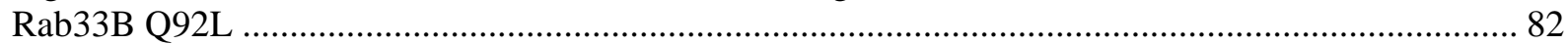

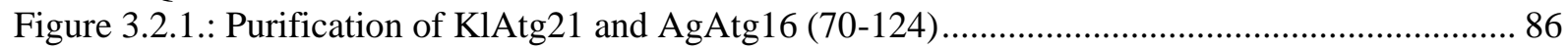

Figure 3.2.2.: Analytic gel filtration of KlAtg21 and AgAtg16 (40-124) .......................................... 87

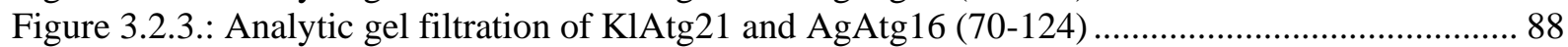

Figure 3.2.4.: Analytic gel filtration of KlAtg21 and AgAtg16 (47-103) ........................................ 89

Figure 3.2.5.: SEC of K1Atg21, AgAtg16 (70-124) alone and KlAtg21-AgAtg16 (70-124) complex. 90

Figure 3.2.6.: MALLS measurements of KlAtg21, AgAtg16 (70-124) and KlAtg21-AgAtg16 (70-124)

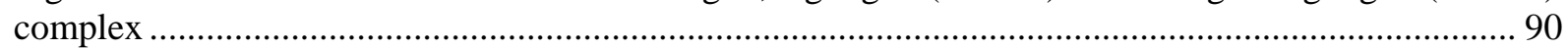

Figure 3.2.7.: Isothermal titration calorimetry titration curves of KlAtg21 with AgAtg16(40-124) .... 91

Figure 3.2.8.: Grid screen of a K1Atg21-AgAtg16 (40-124) crystal ................................................. 92

Figure 3.2.9.: First round in the molecular replacement structure determination of K1Atg21-AgAtg16

Figure 3.2.10.: First round of molecular replacement for Atg21 reveals additional electron density ... 95

Figure 3.2.11.: Refinement statistic plots for the KlAtg21-AgAtg16(40-124) complex....................... 97

Figure 3.2.12.: Superimposition of PaAtg18, KlHsv2 and KmHsv2 ................................................ 98

Figure 3.2.13.: Structure based sequence alignment of yeast PROPPINs ......................................... 100

Figure 3.2.14.: ConSurf analysis of PaAtg 18 and KmHsv2 for conserved Atg21 residues................ 101

Figure 3.2.15.: Crystal packing in the KlAtg21-AgAtg16 crystal.................................................... 102

Figure 3.1.16.: Electron density map of the K1Atg21-AgAtg16 complex crystals .............................. 103

Figure 3.1.17.: Overview of the KlAtg21-AgAtg16 crystal structure ............................................. 104

Figure 3.1.18.: Proposed model for KlAtg21-AgAtg16 interaction................................................. 104

Figure 3.2.19.: Hypothesis for KlAtg21 and AgAtg16 complex formation........................................ 105

Figure 3.2.20.: Analytic gel filtration of KlAtg21 and AgAtg16 (70-124) ..................................... 106

Figure 3.2.21.: Analytic gel filtration of KlAtg21 R103E and AgAtg16 (70-124) ............................ 107

Figure 3.2.22.: Analytic gel filtration of KlAtg21 and AgAtg16 (70-124) D78R............................. 107

Figure 3.2.23.: Analytic gel filtration of KlAtg21 R103E and AgAtg16 (70-124) D78R ................. 108

Figure 3.2.24.: Hypothetical model of Atg21 bound Atg12 Atg5/Atg16 complex interacting with Atg8

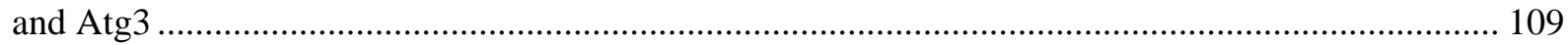

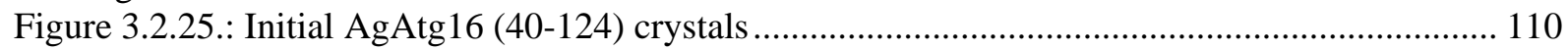

Figure 3.2.26.: Schägger gel electrophoresis of different KlAtg21-AgAtg16 (40-124) purification

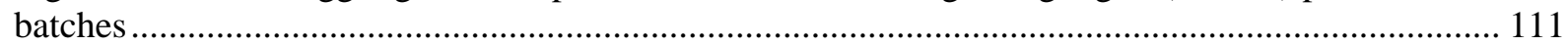

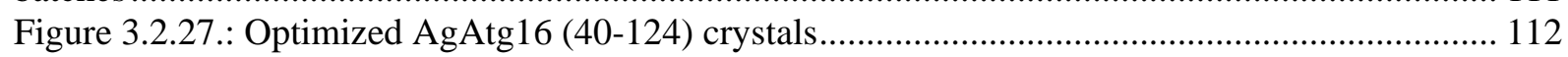

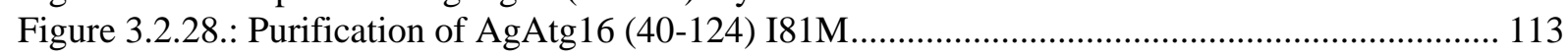

Figure 3.2.29.: Analytic gel filtration of KlAtg21 and AgAtg16 (40-124) I81M ............................. 113

Figure 3.2.30.: AgAtg16 (40-124) crystal mounted at the Swiss Light Source ................................. 114

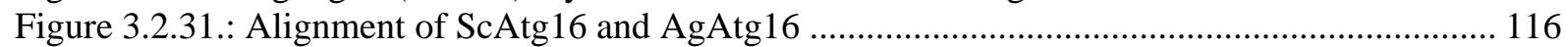

Figure 3.2.32.: Refinement statistic plots for AgAtg16 (40-124) …............................................ 118

Figure 3.2.33.: Four AgAtg16 molecules in the asymmetric unit ................................................ 119

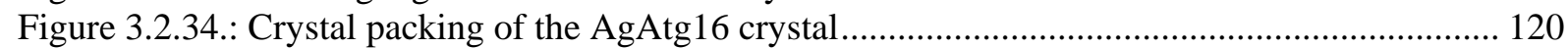

Figure 3.2.35.: Electrostatic potential surface for AgAtg16 dimer AB .......................................... 120

Figure 3.2.36.: Helical wheel projection of the residues in the AgAtg16 dimer AB ......................... 121

Figure 3.3.1.: Purification of SCOC (78-159) FEZ1 (227-290) complexes ...................................... 123

Figure 3.3.2.: Optimized purification of the SCOC (78-159)-FEZ1 (225-295) complex ................... 124

Figure 3.3.3.: CD spectroscopy of the SCOC(78-159)-FEZ1 (225-295) complex .............................. 125 
Figure 3.3.4.: SEC-MALLS of SCOC(78-159)-FEZ1(225-295) complex......................................... 126

Figure 3.3.5.: Initial crystals from SCOC(78-159)-FEZ1(227-290) crystallization............................ 128

Figure 3.3.6.: Initial crystals from SCOC(78-159)-FEZ1(225-295) crystallization........................... 129

Figure 3.3.7.: Elongated hexagonal SCOC(78-159)-FEZ1(225-295) crystals from grid screen

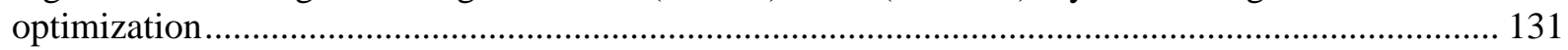

Figure 3.3.8.: Elongated hexagonal SCOC(78-159)-FEZ1(225-295) crystals from drop size

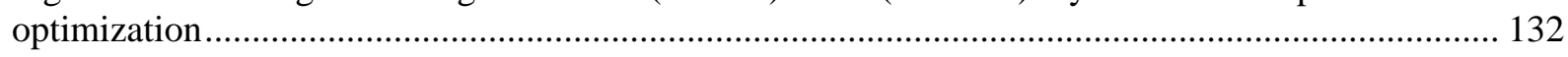

Figure 3.3.9.: SCOC(78-159)-FEZ1(225-295) crystals from additive screen .................................... 133

Figure 3.3.10.: SCOC(78-159)-FEZ1(225-295) crystals after manual dehydration ........................... 134

Figure 3.3.11.: Limited proteolysis of SCOC(78-159)-FEZ1(225-295) complex.............................. 135

Figure 3.3.12.: In situ crystallization of SCOC(78-159)-FEZ1(225-295) ....................................... 136

Figure 3.3.13.: Diffraction pattern of SCOC(78-159)-FEZ1(225-295) crystals at beamline BL14.3 . 137

Figure 3.3.14.: Fluorescence spectrum of selenomethionine labeled SCOC(78-159)-FEZ1(225-295)

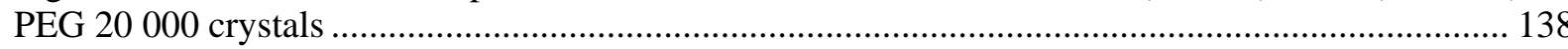

Figure 3.3.15.: SCOC(78-159)-FEZ1(225-295) crystals grown in PEG 20000 conditions at beamline

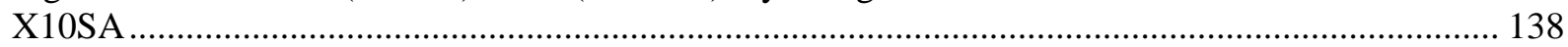

Figure 3.3.16.: SCOC(78-159)-FEZ1(225-295) crystals grown in isopropanol conditions at beamline

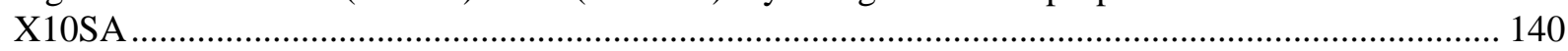

Figure 3.3.17.: Schägger gel electrophoresis of Ni-Sepharose purified SCOC(78-124)-FEZ1(225-295)

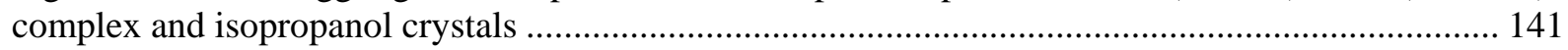

Figure 3.3.18.: Fluorescence spectrum of selenomethionine labeled SCOC(78-159)-FEZ1(225-295)

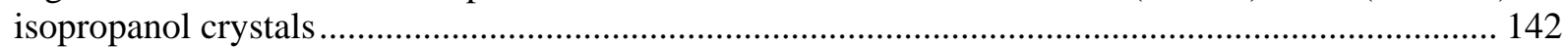

Figure 3.3.19.: Britton analyses from Phenix Xtriage for SCOC-FEZ1 isopropanol crystals ............ 143

Figure 3.3.20: L test for acentric data from Phenix Xtriage for SCOC-FEZ1 isopropanol crystals ... 143

Figure 3.3.21.: Cross-linking of the SCOC(78-159)-FEZ1(225-295) complex................................. 144

Figure 3.3.22.: Visualization of the mass spectrometry analysis of the cross-linking of the SCOC(78-

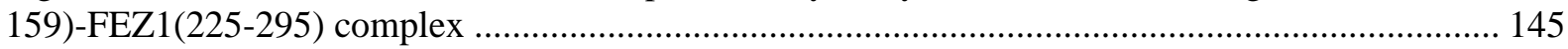

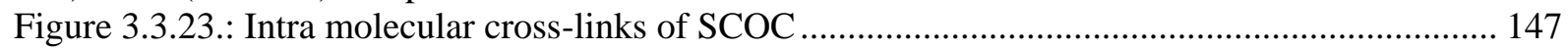

Figure 3.3.24.: Intra molecular cross-links of parallel FEZ1 model ............................................... 148

Figure 3.3.25.: Intra molecular cross-links of antiparallel FEZ1 model .......................................... 148

Figure 3.3.26.: Inter molecular cross-links of SCOC and a parallel coiled coil FEZ1 model.............. 150

Figure 4.1.: Ni-Sepharose pull down of His-Rab33B(30-202)Q92L co-expressed with different

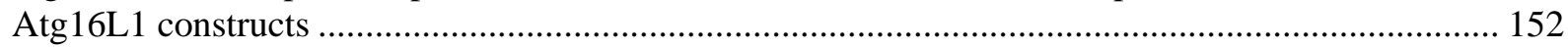

Figure 4.2.: GFP co-immunoprecipitation of Atg16L1-EmGFP and V5-Rab33B variants ................ 156

Figure 4.3.: Hypothetical model of the assembly of the autophagy ubiquitin-like conjugation system at

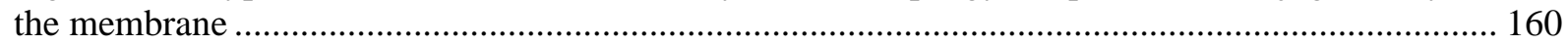

\section{List of tables}

Table 1.1.1.: Overview of name und function of autophagy core machinery proteins in yeast and

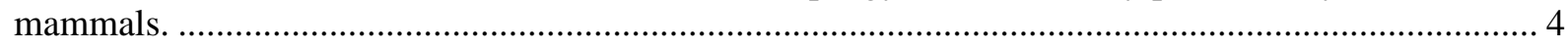

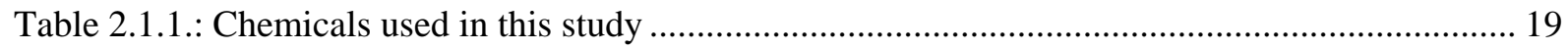

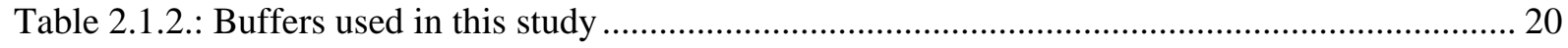

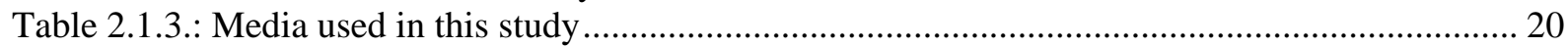

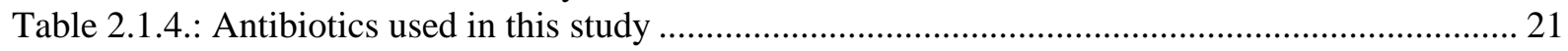

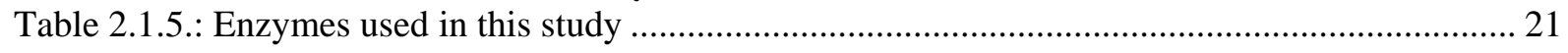

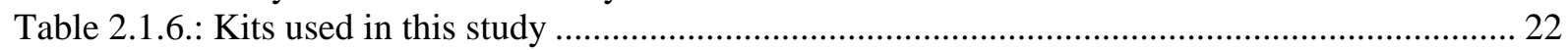

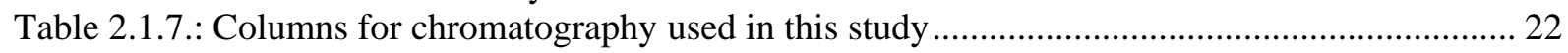

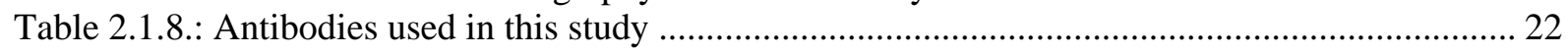

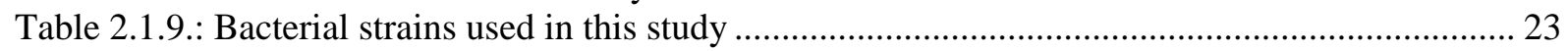

Table 2.1.10.: DNA constructs in single cloning site vectors for bacterial expression......................... 23

Table 2.1.11.: DNA constructs in pET-Duet1 vector for bacterial expression....................................... 24

Table 2.1.12.: DNA constructs in Gateway vectors for mammalian expression................................... 24

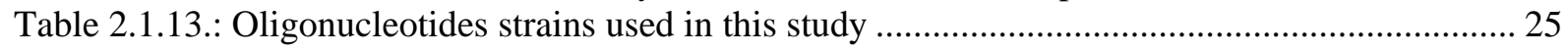




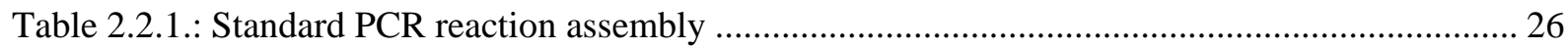

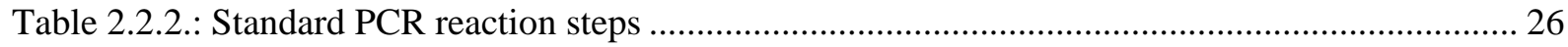

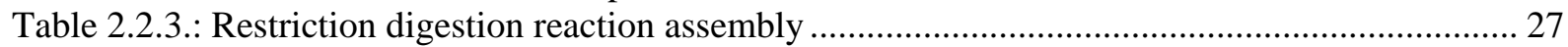

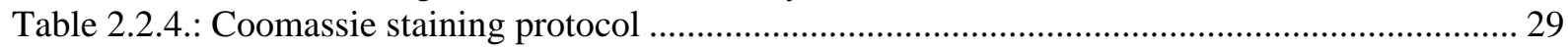

Table 3.1.1.: Initial crystallization conditions for Rab33B(30-202)Q92L-Atg16L1(153-210) from 96-

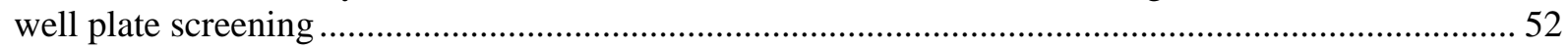

Table 3.1.2.: Data collection for Rab33B(30-202)Q92L-Atg16L1(153-210) crystal ........................... 55

Table 3.1.3.: Matthews coefficient for Rab33B(30-202)Q92L-Atg16L1(153-210) crystals ............... 55

Table 3.1.4.: Results of first round of molecular replacement with Phaser_MR for the Rab33B-

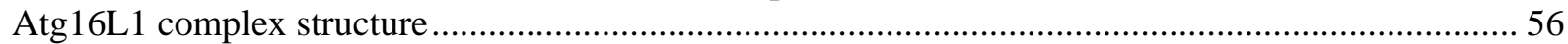

Table 3.1.5.: Result of second round of molecular replacement with Phaser_MR for the Rab33B-

Atg16L1 complex structure ...................................................................................................... 57

Table 3.1.6.: Rab33B(30-202)Q92L-Atg16L1(153-210) residues modeled as alanine in the crystal

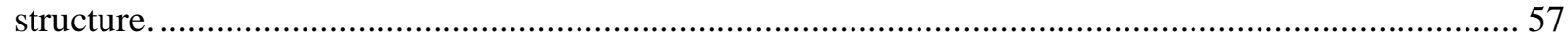

Table 3.1.7.: Data collection and refinement statistics of Rab33B(30-202)Q92L-Atg16L1(153-210)

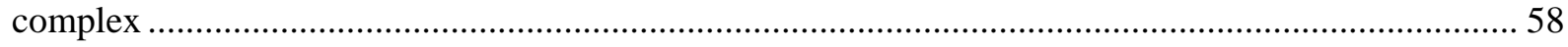

Table 3.1.8.: Data of the mass spectrometry analysis of the cross-linking of the Rab33B-Atg16L1

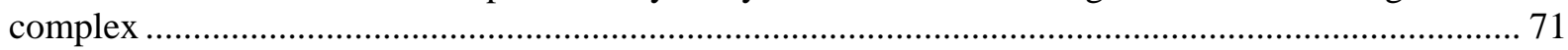

Table 3.2.1.: Molecular weights of KlAtg21, AgAtg16 (70-124) and KlAtg21-AgAtg16 (70-124)

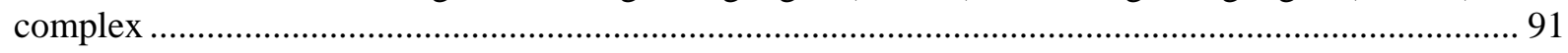

Table 3.2.2.: Data collection for the KlAtg21-AgAtg16(40-124) complex ....................................... 93

Table 3.2.3.: Result of first round of molecular replacement with Phaser_MR for the KlAtg21-

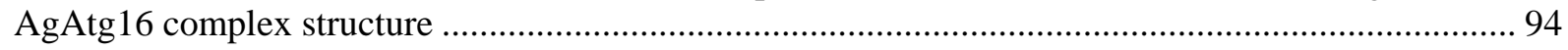

Table 3.2.4.: Result of second round of molecular replacement with Phaser_MR for the KlAtg21-

AgAtg16 complex structure ..................................................................................................... 96

Table 3.2.5.: Data collection and refinement statistics for the KlAtg21-AgAtg16(40-124) complex .. 96

Table 3.2.6.: Comparison of space group and cell dimensions ........................................................ 111

Table 3.2.7.: Data collection for AgAtg16(40-124) crystal .............................................................. 115

Table 3.2.8.: Matthews coefficient for AgAtg16(40-124) crystals ................................................... 115

Table 3.2.9.: Data collection and refinement statistics of AgAtg16 (40-124)................................... 117

Table 3.3.1.: SEC-MALLS measurements of SCOC(78-159)-FEZ1(225-295) complex .................. 127

Table 3.3.2.: Initial crystallization conditions for SCOC(78-159)-FEZ1(227-290) crystallization from

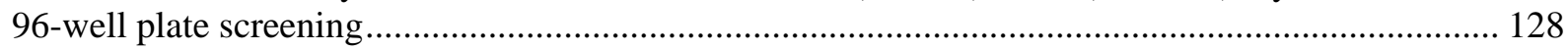

Table 3.3.3.: Initial crystallization conditions for SCOC(78-159)-FEZ1(225-295) crystallization from

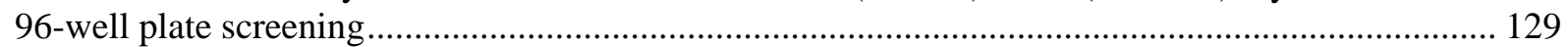

Table 3.3.4.: Optimization approaches for SCOC(78-159)-FEZ1(225-295) crystallization ............... 130

Table 3.3.5.: Crystallization conditions for SCOC(78-159)-FEZ1 (225-295) crystals from grid screen optimization .................................................................................................................. 131

Table 3.3.6.: Crystallization conditions for SCOC(78-159)-FEZ1(225-295) crystals from drop size

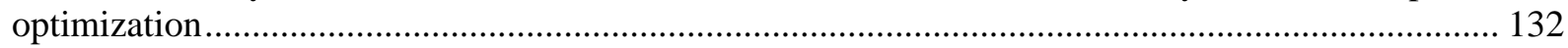

Table 3.3.7.: Determination of lattice character and bravais lattice from XDS IDXREF for SCOC-

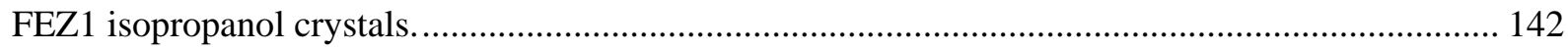

Table 3.3.8.: Data of the mass spectrometry analysis of the SCOC(78-159)-FEZ1(225-295) complex

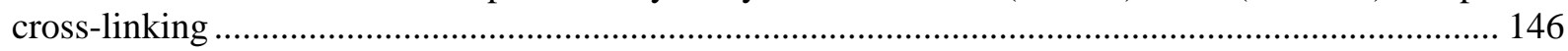




\section{Introduction}

\subsection{Overview on autophagy}

Autophagy (Greek for "self eating") unites a number of highly conserved cellular processes that involve the degradation of cytoplasmic components in the yeast vacuole or lysosomes in animal cells. Autophagy occurs from yeast to humans. It plays an important role in cell survival and maintenance, during starvation parts of the cytoplasm and organelles are unspecific degraded to recover energy and to gain building blocks that are recycled for protein synthesis (Fig. 1.1.1.) [113]. To maintain cell homeostasis autophagy is functional in the specific degradation of protein aggregates and damaged organelles like mitochondria, peroxisomes or the endoplasmic reticulum (ER) that are too large to be handled by the ubiquitin-proteasome pathway [64, 65]. In addition autophagy can remove intracellular pathogens $[38,89]$.

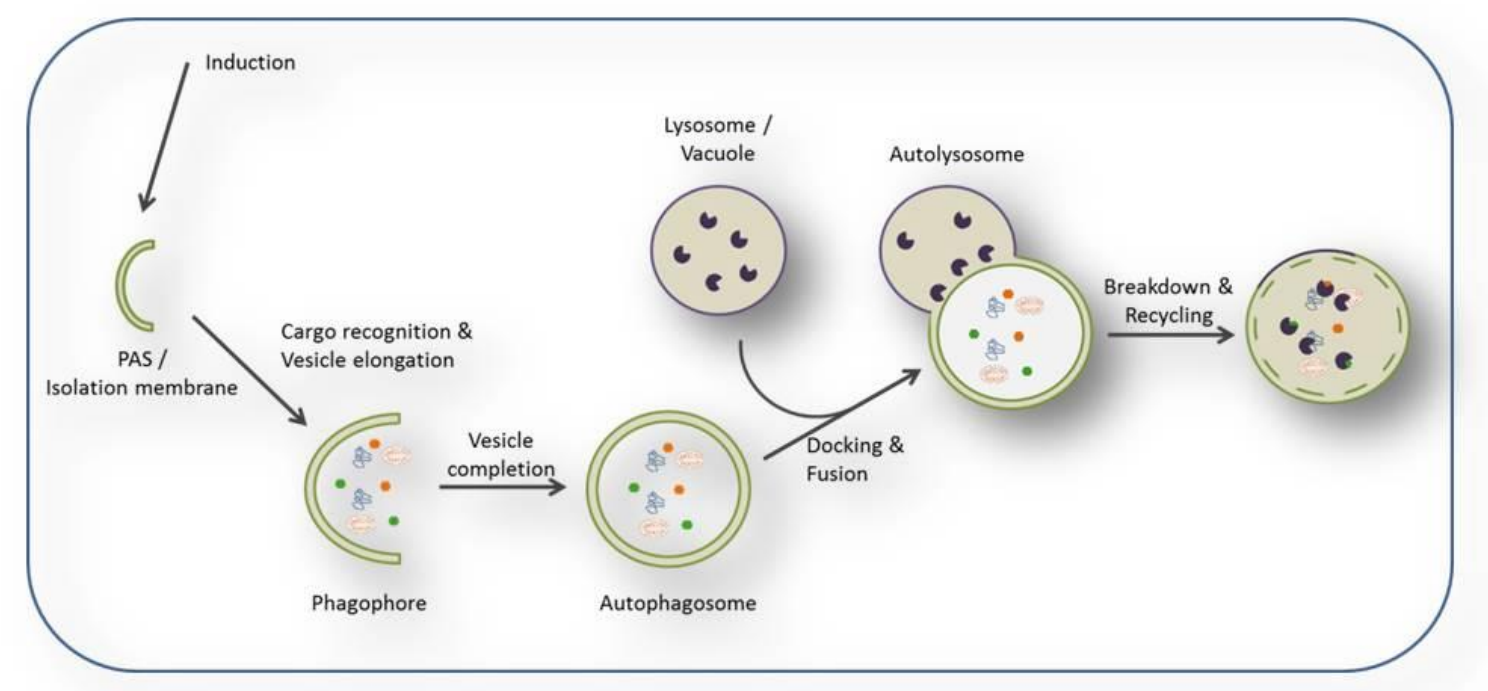

Figure 1.1.1.: Schematic overview on macroautophagy. Upon induction de novo formation of an isolation membrane occurs at the preautophagosomal assembly site (PAS). The expanding double membraned phagophore engulfs its cargo and then closes to form the autophagosome. Upon fusion with the lysosome or vacuole the inner autophagosomal membrane and cargo are degraded and recycled.

There are two main types of non-specific autophagy: microautophagy and macroautophagy (Fig. 1.1.2.). During microautophagy cytoplasm including organelles is taken up directly through invaginations of the lysosome or vacuole (Fig. 1.1.2) [77]. Macroautophagy on the 
other hand sequesters cytoplasmic cargo with a de novo growing isolation membrane or phagophore (Fig. 1.1.1. and 1.1.2.). Closing of this double-membrane forms the autophagosomes that are delivered to the lysosome where they fuse. The inner membrane of the autophagosome and its content are then degraded [125]. Macroautophagy is the best studied autophagy pathway and will be described in more detail below. The cytoplasm-tovacuole targeting $(\mathrm{Cvt})$ pathway and piecemeal microautophagy of the nucleus (PMN) in yeast and chaperone-mediated autophagy in mammalian cells are three examples for specific autophagic subtypes. The cytoplasm to vacuole targeting $(\mathrm{Cvt})$ pathway is a biosynthetic process that uses the autophagy machinery. It delivers the hydrolases $\alpha$-mannosidase (Ams1) and the precursor form of aminopeptidase I (preApe1) to the vacuole [80]. PMN is a starvation induced process that degrades small portions of the nucleus at nucleus-vacuole (NV) junctions [71, 117]. Chaperone-mediated autophagy (CMA) is a highly specific nonvesicular form of autophagy. Heat shock cognate protein of $70 \mathrm{kDa}$ (hsc70) recognizes a KFERQ related pentapeptide motif in substrates and delivers them to the lysosome, where they bind to the lysosome-associated membrane protein type 2A (LAMP-2A) receptor. Substrates are then translocated directly across the lysosomal membrane [62]. Other autophagic subtypes are known which specifically target organelles, for example peroxisomes (pexophagy), mitochondria (mitophagy), and the endoplasmic reticulum (ER-phagy), reviewed in [35]. Xenophagy targets invading pathogens and is part of the cellular defense mechanism [66]. 


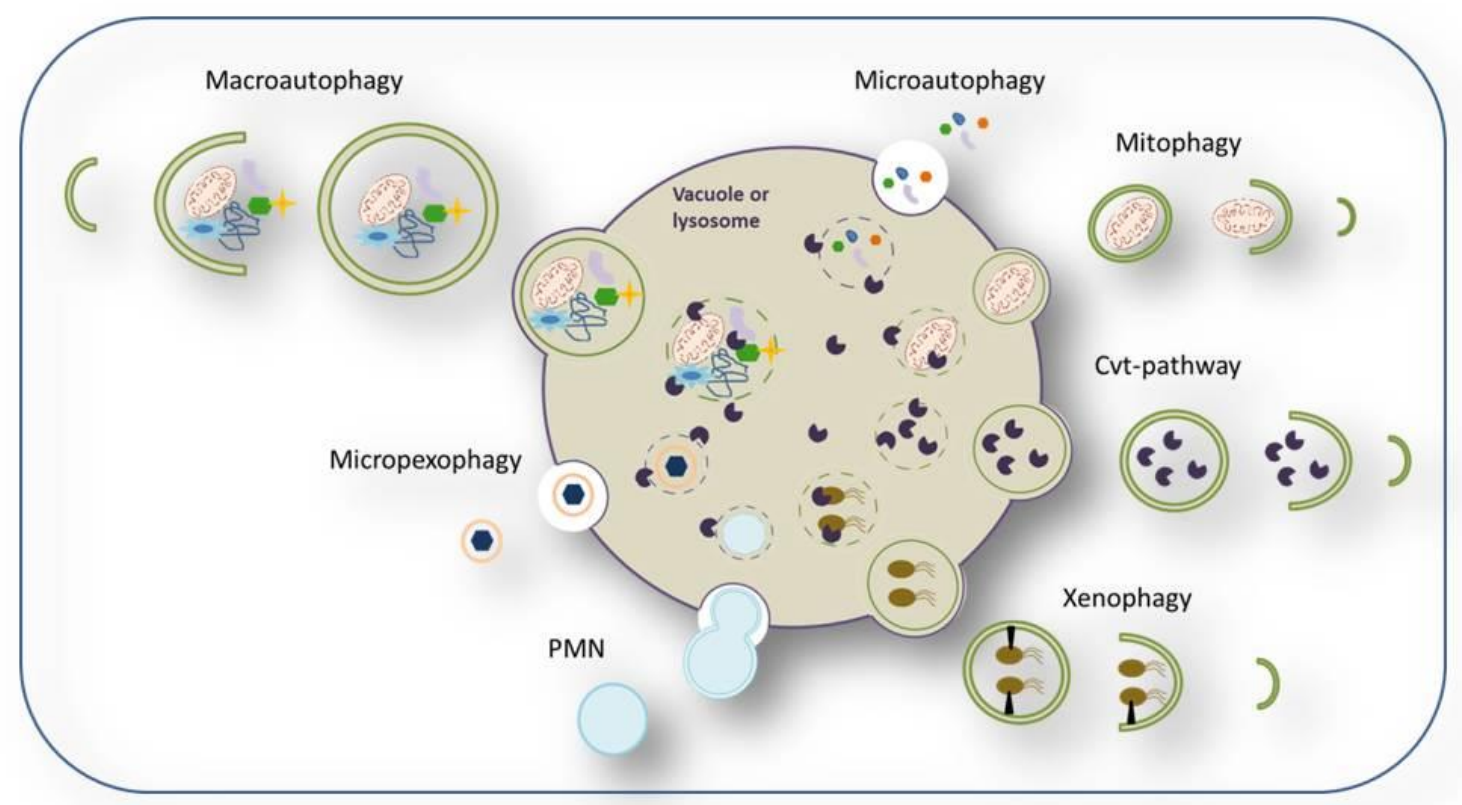

Figure 1.1.2.: Schematic overview of autophagy subtypes and their morphology. Macro- and microautophagy are two types of non-selective autophagy. During macroautophagy a portion of the cytoplasm is sequestered into a double-membraned vesicle, the autophagosome, which then fuses with the vacuole or lysosome. Selective autophagy subtypes include mitophagy, which takes up mitochondria, xenophagy for pathogens and the biosynthetic cytoplasm to vacuole targeting (Cvt) pathway that delivers hydrolases to the vacuole. During microautophagy cargo is taken up directly through invaginations of the vacuolar or lysosomal membrane and a similar selective subtype is micropexophagy that specifically targets peroxisomes. Piecemeal microautophagy of the nucleus (PMN) degrades portions of the nucleus. Both yeast and mammalian autophagic subtypes are shown in this figure.

Macroautophagy, hereafter named autophagy, occurs at a low basal level constitutively under normal growing conditions [89] and is strongly upregulated under stress conditions like nutrient starvation. Importantly, autophagy degrades organelles and aggregated proteins that are too large for the ubiquitin-proteasome system. It encloses them in autophagosomes that have a size of 400-900 nm [135]. The double-membraned autophagosomes do not form by budding from other organelles but by de novo formation at the preautophagosomal assembly site (PAS). The cup-shaped initial sequestering compartment is called the isolation membrane or phagophore [133]. The phagophore grows by the addition of lipids, most likely via vesicle fusion mediated by SNAREs (soluble N-ethylmaleimide-sensitive fusion (NSF) attachment protein receptors) [142]. The membrane supply involves various sources at different stages and is still controversially discussed [112]. Different studies gave hints that lipid bilayers are either delivered from the ER [80], Golgi apparatus [43, 82] or other compartments including the plasma membrane [111] and mitochondria [47]. At the end the strongly bent phagophore 
closes and forms the autophagosome [68]. Autophagosomes are trafficked to the lysosome or vacuole where their outer membranes fuse with the lysosome or vacuole [79]. The membrane of the remaining vesicle, the autophagic body in yeast, is lysed and the content is degraded by hydrolases and recycled [7].

Autophagy genes were discovered by pioneering yeast genetic screens performed independently by the groups of Yoshinori Ohsumi, Michael Thumm and Daniel Klionsky [51, 137, 138] and are denoted as ATG (autophagy-related) genes. Currently, more than 40 Atg proteins are known [78]. The majority of autophagy related proteins assemble into complexes. Eighteen of them form the core autophagy machinery which is essential for the autophagosome formation [97]. Many aspects of autophagy are conserved from yeast to human and most yeast Atg proteins have orthologues in mammals (see Table 1.1.1. for comparison of the yeast and mammalian core autophagy machinery).

Table 1.1.1.: Overview of name und function of autophagy core machinery proteins in yeast and mammals. Abbreviation: nd, no data. Modified from [13].

\begin{tabular}{|c|c|c|c|}
\hline & Yeast & Mammals & Function \\
\hline \multirow{6}{*}{$\begin{array}{l}\text { Atg1 kinase } \\
\text { complex }\end{array}$} & Atg1 & ULK1, 2 & Serine/threonine protein kinase \\
\hline & $\operatorname{Atg} 13$ & ATG13 & Target of the TOR signaling pathway \\
\hline & $\operatorname{Atg} 17$ & FIP200 & $\begin{array}{l}\text { Scaffold protein, regulatory subunit of the } \\
\text { complex }\end{array}$ \\
\hline & nd & ATG101 & $\begin{array}{l}\text { Forms a complex with ULK, ATG13 and } \\
\text { FIP200 in mammals, function unknown }\end{array}$ \\
\hline & $\operatorname{Atg} 29$ & $\mathrm{Nd}$ & $\begin{array}{l}\text { Forms a complex with Atg17 and Atg } 31 \text { in } \\
\text { yeast, that serves as scaffold for Atg1 } \\
\text { complex assembly }\end{array}$ \\
\hline & $\operatorname{Atg} 31$ & $\mathrm{Nd}$ & $\begin{array}{l}\text { Forms a complex with Atg17 and Atg29 in } \\
\text { yeast, that serves as scaffold for Atg1 } \\
\text { complex assembly }\end{array}$ \\
\hline \multirow[t]{3}{*}{$\begin{array}{l}\text { Phosphatidylinositol } \\
\text { 3-kinase complex }\end{array}$} & Vps34 & PIK3C3/Vps34 & $\begin{array}{l}\text { Class III Phosphatidylinositol 3-kinase, } \\
\text { produces PI(3)P }\end{array}$ \\
\hline & $\operatorname{Atg} 6 / \operatorname{Vps} 30$ & Beclin1 & Forms a complex with Vps34 and Atg14 \\
\hline & Atg14 & ATG14L1 & Forms a complex with Vps34 and Atg6 \\
\hline \multirow[t]{3}{*}{ Atg9 complex } & Atg9 & mATG9 & $\begin{array}{l}\text { Transmembrane protein, carrier for } \\
\text { membranes during phagophore expansion }\end{array}$ \\
\hline & Atg2 & ATG2A, B & $\begin{array}{l}\text { Forms a complex with Atg18, that recruits } \\
\text { Atg9 }\end{array}$ \\
\hline & $\operatorname{Atg} 18$ & WIPI-1, 2 & $\begin{array}{l}\text { Forms a complex with Atg2, that recruits } \\
\text { Atg9 }\end{array}$ \\
\hline
\end{tabular}




\begin{tabular}{|l|l|l|l|}
\hline $\begin{array}{l}\text { Ubiquitin-like } \\
\text { conjugation systems }\end{array}$ & Atg3 & ATG3 & E2-like enzyme, involved in LC3 lipidation \\
\cline { 2 - 4 } & Atg4 & $\begin{array}{l}\text { ATG4A, B, C, } \\
\text { D }\end{array}$ & $\begin{array}{l}\text { Cysteine protease, involved in LC3 activation } \\
\text { and delipidation }\end{array}$ \\
\cline { 2 - 4 } & Atg5 & ATG5 & $\begin{array}{l}\text { Part of the Atg12 Atg5/Atg16 complex, E3- } \\
\text { like enzyme, involved in LC3 lipidation }\end{array}$ \\
\cline { 2 - 4 } & Atg7 & ATG7 & $\begin{array}{l}\text { E1-like enzyme, involved in LC3 and ATG12 } \\
\text { conjugation }\end{array}$ \\
\cline { 2 - 4 } & Atg8 & $\begin{array}{l}\text { LC3A, B, C; } \\
\text { GABARAPs; } \\
\text { GATE-16 }\end{array}$ & $\begin{array}{l}\text { Ubiquitin-like proteins, that are conjugated to } \\
\text { PE }\end{array}$ \\
\cline { 2 - 4 } & Atg10 & ATG10 & E2-like enzyme in Atg12 conjugation \\
\cline { 2 - 4 } & Atg12 & ATG12 & $\begin{array}{l}\text { Ubiquitin-like protein, that conjugates to } \\
\text { Atg5 }\end{array}$ \\
\cline { 2 - 4 } & Atg16 & ATG16L1,2 & $\begin{array}{l}\text { Part of the Atg12 Atg5/Atg16 complex, } \\
\text { specifies LC3 lipidation site }\end{array}$ \\
\hline
\end{tabular}

\subsection{Molecular mechanism of autophagy}

Autophagy initiation is an answer to nutrient starvation by inhibition of the TOR (target of rapamycin) kinase. Inhibition of TOR leads to Atg13 dephosphorylation, which then binds Atg1 kinase [60]. Activated Atg1 and Atg13 interact with the Atg17-Atg29-Atg31 complex at the preautophagosomal assembly site (PAS) [81]. The Atg1 complex recruits downstream Atg proteins to the PAS [106]. One of the first is the autophagy-specific phosphatidylinositol 3kinase complex (Vps30/Atg6, Atg14, Vps15, and Vps34) that is essential for autophagosome formation and produces phosphatidylinositol 3-phosphate (PI(3)P) (Fig. 1.2.1.) [123, 147]. $\mathrm{PI}(3) \mathrm{P}$ is required for the autophagic pathway. It recruits effectors like PROPPINs ( $\beta$ propeller proteins that bind polyphosphoinositides) and anchors them to the membrane [18]. Atg18 is a PROPPIN that forms a complex with Atg2 and is involved in the cycling of Atg9 [101]. Atg9 is the only membrane spanning Atg protein and is integrated into Golgi-derived vesicles that are proposed to deliver lipids to the growing isolation membrane [114]. Two ubiquitin-like conjugation systems are essential for autophagosome formation. The ubiquitinlike protein Atg12 is conjugated to Atg5 by Atg7, an E1-like enzyme and Atg10, an E2-like enzyme that forms a bond between Atg12 and Atg5. The Atg12 Atg5 conjugate forms a stable complex with self-dimerizing Atg16, which binds to the outer side of the growing isolation membrane [139]. Binding of dimeric Atg12 Atg5/Atg16 to the PAS defines site of autophagosome formation and acts as a E3-like ligase second ubiquitin-like conjugation system [99]. Here, the ubiquitin-like Atg8, that is evenly distributed at the isolation membrane (Fig. 1.2.1.), is conjugated by Atg7 and Atg3 the respective E1-like and E2-like enzymes to phosphatidylethanolamine (PE). Atg8-PE is one of the major phospholipids that supports 
membrane extension [96] and assembles with the Atg12 Atg5/Atg16 complex to form a scaffold at the outside of the isolation membrane (Fig. 1.2.1.) [61]. Lipidation of Atg8 is reversed by cleavage of the Atg4 protease during nutrient-rich conditions. Atg8-PE is able to facilitate membrane tethering and hemifusion and has an essential role in autophagosome formation $[95,96]$. The closing of the isolation membrane, the autophagosome formation, is poorly understood. Shortly before or after autophagosome formation the Atg12 Atg5/Atg16 complex dissociates from the surface and Atg8 is released through cleavage of Atg8-PE by Atg4 [63]. The autophagosome then fuses with the lysosome.

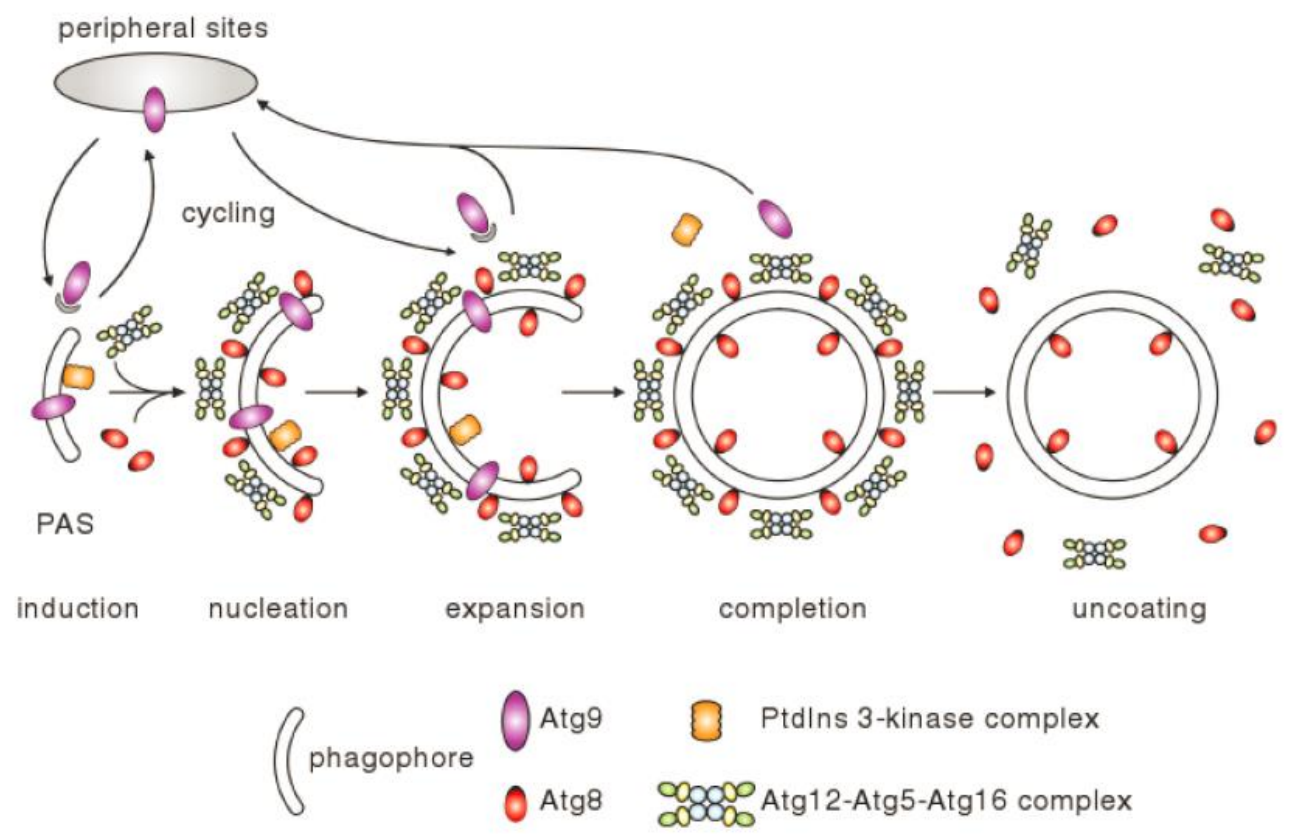

Figure 1.2.1.: Schematic overview of the molecular mechanisms of autophagy. While the phagophore nucleates at the PAS, Atg9 and the PtdIns3K complex are recruited. Growing of the phagophore is organized by several autophagy complexes. The PtdIns $3 \mathrm{~K}$ complex produces PI(3)P and Atg9 cycles between the PAS and peripheral sites to deliver lipids. The Atg12 Atg5/Atg16 complex binds to the outer side of the phagophore and conjugates the evenly distributed Atg8 to Atg8PE. Upon completion of the autophagosome, autophagy proteins are cleaved from the outer membrane and the mature vesicle can fuse with the vacuole. Figure from [146].

The structures of the components of the two ubiquitin-like conjugation systems are known (Fig.1.2.2). The Atg12 Atg5/Atg16 complex is homodimeric and consists of two $\operatorname{Atg} 12 \sim \operatorname{Atg} 5$ conjugates that are bound to one chain of an Atg16 dimer respectively. The Atg12 structure reveals a C-terminal ubiquitin-fold including the typical conserved motif of five-stranded $\beta$-sheet and two $\alpha$-helices, although Atg12 has no sequence homology to ubiquitin (Fig. 1.2.2. A) [42, 134]. Human Atg12 residue G140 is covalent linked to Atg5 
K130 [99, 102]. Atg5 comprises two ubiquitin-like domains that flank a helix-rich domain [83]. It binds $\operatorname{Atg} 12$ on one side of the molecule whereas Atg16 is bound on the other site. Atg12 and Atg16 do not interact directly. Besides its N-terminal Atg5-binding domain, Atg16 comprises a C-terminal coiled coil domain that facilitates homodimerization (Fig. 1.2.2. B) [37], mammalian Atg16L additional contains seven WD repeats at the C-terminus [90]. The Atg12 5 conjugate promotes the conjugation of Atg8 to phosphatidylethanolamine by direct interaction between Atg12 and Atg3 [36]. The Atg8 structure obtains an ubiquitin fold with a central five-stranded $\beta$-sheet, surrounded by two pair of $\alpha$-helices [48, 67]. Before lipidation Atg8 is processed by Atg4 to expose a C-terminal Gly and activated by Atg7 [63].

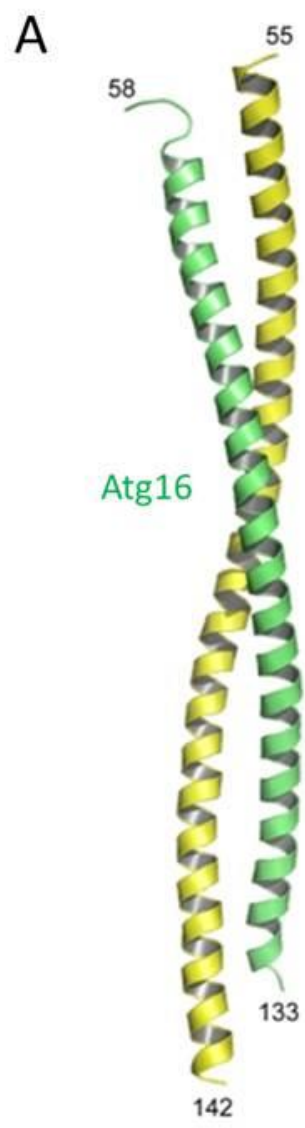

B
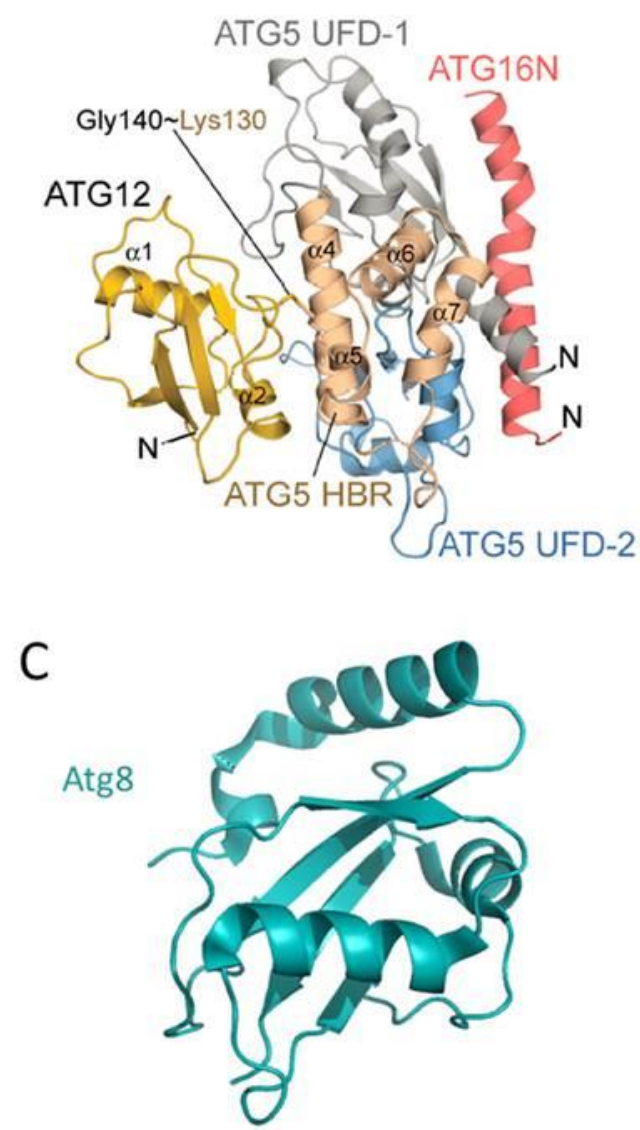

Figure 1.2.2.: Crystal structures of the ATG12 ATG5/ATG16N complex and the Atg16 coiled coil domain. A: Cartoon presentation of yeast Atg 16 forming a parallel coiled-coil dimer (PDB code: 3A7P). B: Cartoon representation of the human ATG12 ATG5 in complex with an N-terminal ATG construct (PDB code: 4GDL). ATG12 colored yellow, ATG16 colored red. ATG5 consists of two ubiquitin folds (UFD-1 in grey and UFD-2 in blue) and a $\alpha$-helical bundle region (HBR in wheat). Atg12 G140 is covalent linked to Atg5 K130. C: Cartoon representation of S. cerevisiae Atg8 in dark turquoise (PDB code: 3VWX, [67]). Figure A from [37], B from [102]. 


\subsection{PROPPINs and the PROPPIN Atg21-Atg16 complex}

Seven natural PIs are known that are specifically recognized by PI effector proteins [8]. The PROPPINs ( $\beta$-propellers that bind polyphosphoinositides) are PI effector proteins that specifically bind $\mathrm{PI}(3) \mathrm{P}$ and $\mathrm{PI}(3,5) \mathrm{P}_{2}$. The conserved FRRG motif is essential for PI binding of PROPPINs [29, 73, 95, 101]. In yeast there are three PROPPIN homologs Atg18, Atg21 and Hsv2 (Homologous with swollen vacuole phenotype 2) [70]. In mammals four PROPPINs were discovered and named WIPI1 to WIPI4 (WD-40 repeat containing protein that interacts with PIs) [109]. WIPI1 and WIPI2 are postulated as Atg18 orthologues with different functions [108]. WIPI1 has similar functions like the yeast Atg18 and localizes to the autophagosomal membrane in a phosphoinositide dependent manner [110]. WIPI2 was often referred to be an orthologue of Atg21 but bioinformatic analyses show that Atg21 is yeast specific and has only distant ancestry with the WIPI family [108]. Still similar to yeast Atg21 that binds Atg16, WIPI2B interacts with Atg16L1 and thereby recruits the Atg12 Atg5/Atg16L complex to facilitate LC3-lipidation in mammals [28, 108]. WIPI3 and WIPI4 feasible are orthologues of Hsv2.

Yeast PROPPINs are highly homologous and structurally conserved. Crystal structures are available for KlHsv2 and KmHsv2 [9, 69, 143] and PaAtg18 [119]. Structural characterization revealed that the conserved FRRG motif is part of two phosphoinositide binding pockets. PROPPINs are WD-40 repeat proteins that fold into a seven-bladed $\beta$-propeller (Fig. 1.3.1. 1-7) [9, 69, 143]. Each blade is comprised of four antiparallel $\beta$ strands (Fig. 1.3.1. A-D from inside to outside). Each $\beta$-strand is connected to the next $\beta$ strand by a loop region. While the $\beta$-strands are conserved within the PROPPINs the loop regions are not conserved and differ in length [73]. 

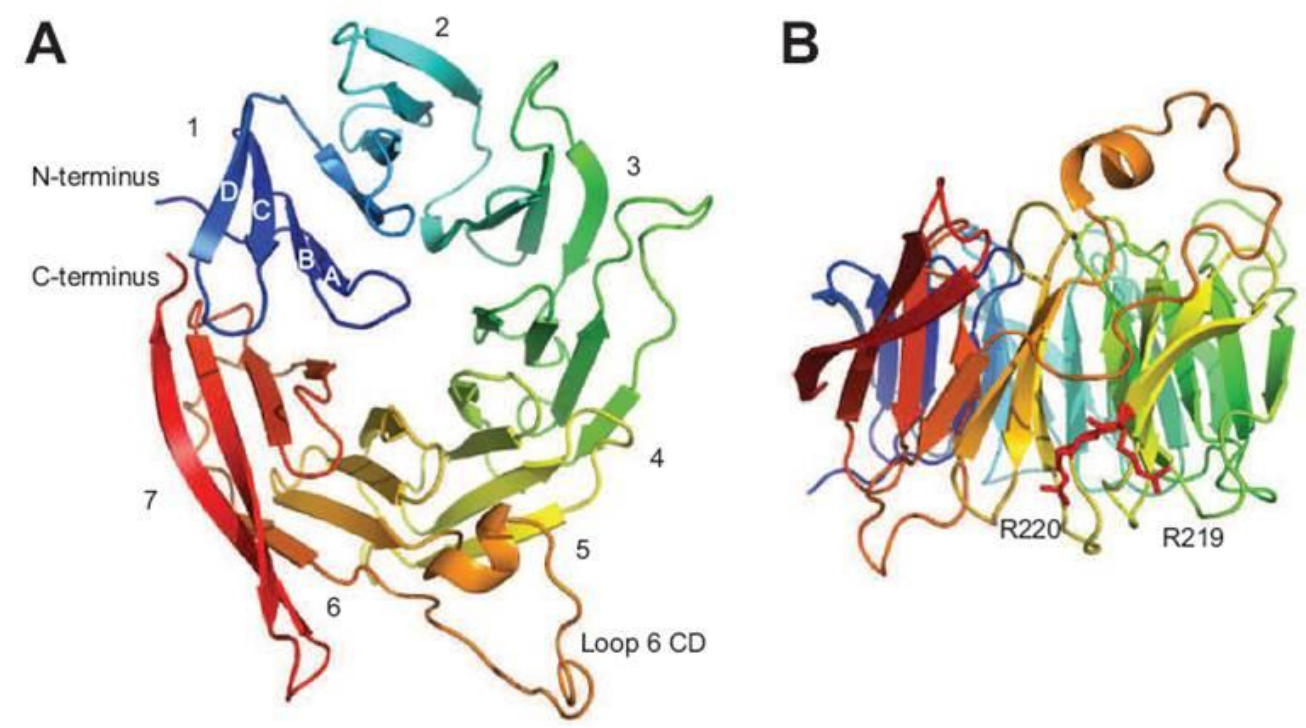

Figure 1.3.1.: Structure of the PROPPIN Kluyveromyces lactis Hsv2. A: Top view of the KlHsv2 structure. PROPPINS form a seven-bladed $\beta$-propeller scaffold (numbers 1-7) each blade consists of four antiparallel $\beta$-strands (letters A-D). B: Side view of the same structure showing the FRRG lipid binding motif (R219 and R220). Cartoon representation, colored in rainbow colors from $\mathrm{N}$-terminal (blue) to C-terminal (red). (PDB: code 4AV9). Figure from [69].

Yeast PROPPINs play a role in different autophagy subtypes. Atg18 is a core autophagy protein and is involved in all autophagy types. In the early stage of autophagy it binds to $\mathrm{PI}(3) \mathrm{P}$ at the PAS and interacts with Atg2 and is involved in mediating Atg9 vesicle cycling [115]. Furthermore Atg18 is essential for retrograde trafficking from the vacuole by binding of $\mathrm{PI}(3,5) \mathrm{P}_{2}$ and maintaining vacuole homeostasis as part of the lipid kinase Fab1 complex $[29,32]$. Hsv2 was so far only described to be involved in piecemeal autophagy of the nucleus (PMN) [70]. Atg21 functions in the selective Cvt pathway [95] and mitophagy [131] and regulates pexophagy in Pichia pastoris [136]. Atg21 binds via PI(3)P to the phagophore and recruits the Atg12 Atg5/Atg16 complex [58]. It has been suggested that $A \operatorname{tg} 21$ binds $A \operatorname{tg} 8$ at the top of the propeller and by recruiting of Atg8 determines the site of Atg8 lipidation [58]. The Atg21 binding sites for Atg8 and Atg16 are distinct. Residues E102 and especially D101 of the Atg16 coiled coil domain are required for Atg21 binding [58]. The group of Michael Thumm proposed a model (Fig. 1.3.2.) where Atg21 binds via PI(3)P to the phagophore and recruits the $\operatorname{Atg} 12 \sim \operatorname{Atg} 5 / \operatorname{Atg} 16$ complex and $A \operatorname{tg} 8$ to the membrane in a distinct position. In this way Atg12-Atg5-Atg16 and Atg3 act as an E3-like enzyme lipidating Atg8 [58]. 


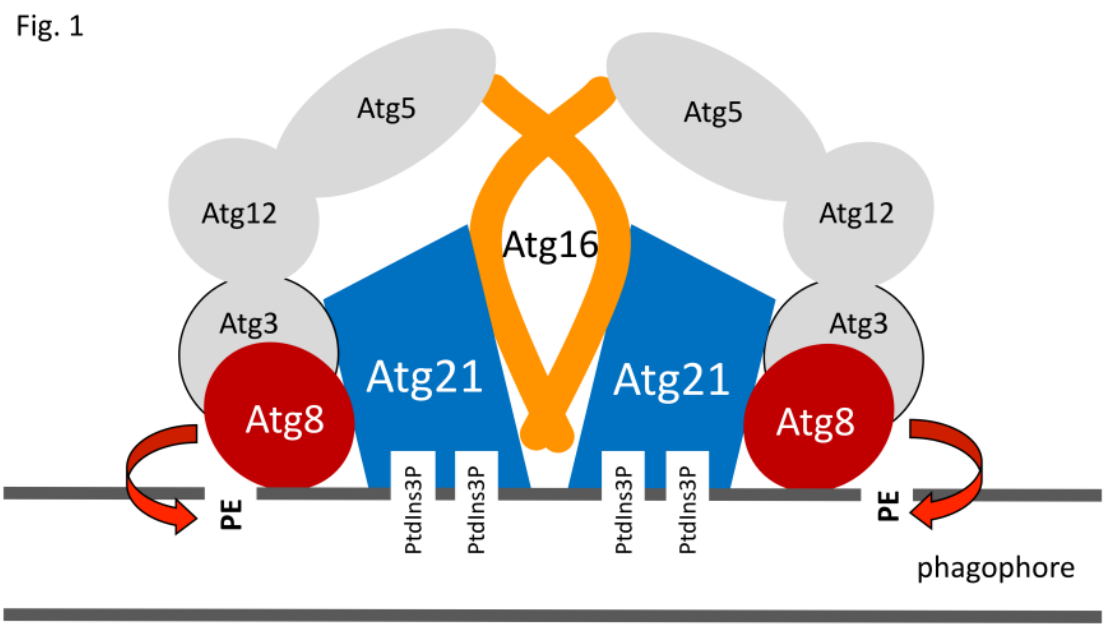

Figure 1.3.2.: Model for the coordination of Atg8 lipidation. Atg2 1 localizes to the phagophore by $\mathrm{PI}(3) \mathrm{P}$ binding, where it interacts with the dimeric $A \operatorname{tg} 12 \sim \operatorname{Atg} 5 / \mathrm{Atg} 16 \mathrm{~L}$ complex and thereby coordinates Atg8 lipidation by Atg3. Figure from [72].

\subsection{The functions of Rab33B and Atg16L1 in autophagy}

Rab GTPases play a crucial role in membrane trafficking processes and several Rab proteins are involved in autophagy (Fig. 1.4.1.) $[24,124]$. Rab proteins (Ras-related in brain) are small GTPases and form the largest family within the Ras superfamily of monomeric G proteins [45]. Rab proteins are present in yeast and mammals.

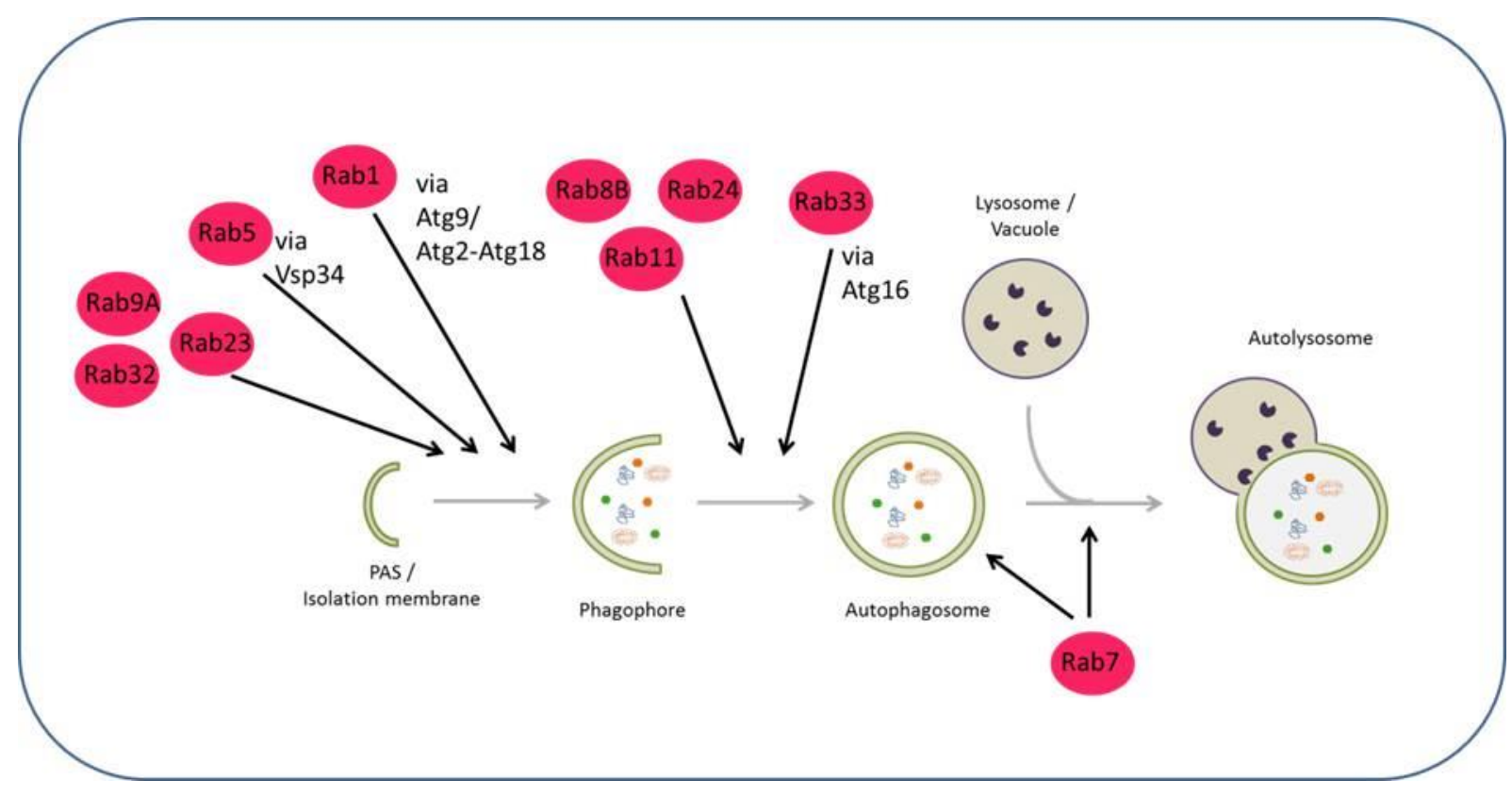

Figure 1.4.1.: Schematic overview of Rab GTPases involvement in autophagy. Rab proteins Rab1, Rab5, Rab9A, Rab23 and Rab32 regulate autophagosome formation. Rab8B, Rab11, Rab24 and Rab33 promote autophagosome maturation. Rab7 regulates both autophagosome maturation and fusion with the lysosome. 
More than 25 Rab proteins have been structurally characterized so far [31]. They share a fold that consists of a six stranded $\beta$-sheet with five parallel $\beta$-strands and one antiparallel $\beta$ strand, surrounded by five $\alpha$-helices (Fig. 1.4.2.). The amino acids of the active site are highly conserved within the entire Ras superfamily [144]. Five loops that connect the $\alpha$-helices with the $\beta$-strands contain the elements for binding of guanine nucleotide exchange factors (GEFs) and $\mathrm{Mg}^{2+}$ and GTP hydrolysis [129]. Rab proteins shuttle between an active state (GTPbound) and an inactive state (GDP-bound). Two regions: switch I (effector domain G-2) and switch II (loop4/ $\alpha$-helix 2 after the G-3 region) undergo major conformational changes between the two states (Fig. 1.4.2.) [88, 122]. A third region encompass the phosphatebinding loop (P-loop or Walker A motif) that is highly conserved through all ATP- or GTPbinding proteins with consensus sequence of GXXXXGKT/S (where $\mathrm{X}$ is any amino acid) [118]. Mutating the catalytic important glutamine in the switch II region, that positions water for nucleophilic attack on the GTP $\gamma$-phosphate to a leucine results in a GTPase deficient mutant that traps the GTPase in a GTP-bound constitutive active form, for example Q92L in Rab33B. The T47N mutation in the P-loop region of Rab33B provides a dominant negative mutant that binds GDP with higher affinity than GTP (Fig. 1.4.2.). 
A

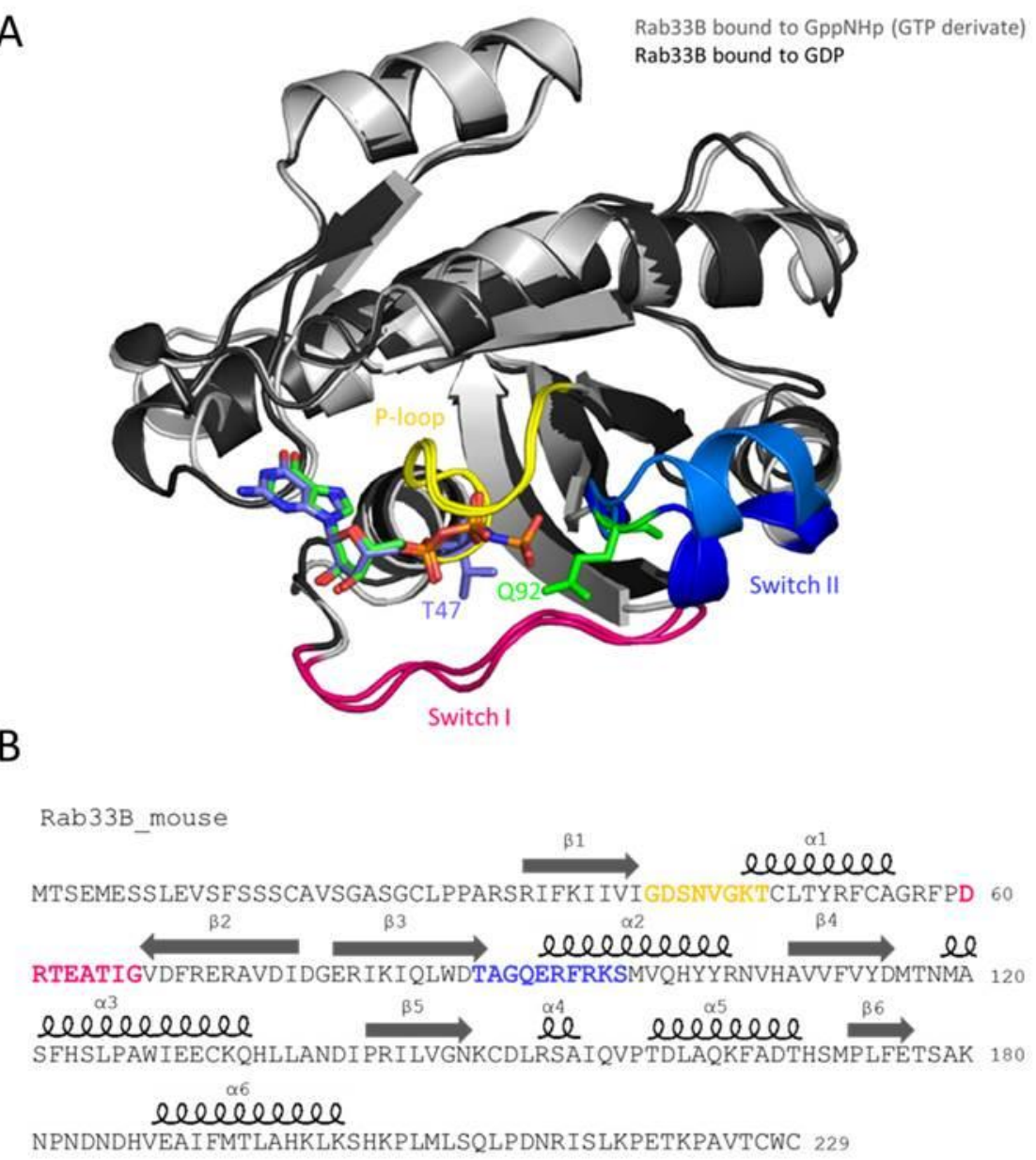

B

Figure 1.4.2.: Rab33B structures in the active and inactive state. A: Cartoon representation of GppNHp-bound Rab33 colored in grey (PDB code: 1Z06) and GDP-bound Rab33 in dark grey (PDB code 2G77). Switch region I colored in pink, P-loop in yellow and Switch II region in blue for GppNHp-bound Rab33 and light blue for GDP-bound Rab33. Stick representation of GppNHp and residue Q92 in green and GDP and residues T47 in smokey blue. B: Amino acid sequence of murine Rab33B with switch I, switch II and P-loop region colored according to A. Secondary structure elements are shown based on the structure of GppNHp-bound Rab33 (PDB code: 1Z06).

Rab proteins function in all steps of autophagy (Fig. 1.4.1., for a review see [4]). Rab proteins Rab1, Rab5, Rab9A, Rab23 and Rab32 contribute to autophagosome formation. Rab7, Rab8B, Rab11 and Rab24 are required for autophagosome maturation. Rab7 and its effector the Vsp34 complex, play a key role in the regulation of autophagosome maturation and fusion with the lysosome [128]. Rab9 is needed in non-canonical autophagy, an Atg5/Atg7independent mode of autophagosome formation [98]. The exact role of Rab8A and Rab25 in autophagy is still unclear. Rab26 has recently been discovered to mediate the turnover of recycled synaptic vesicles by interaction with the Atg16L1-Atg5 complex via the autophagy pathway [14]. In addition, an even stronger direct interaction has been found between Rab33 
and Atg16L1 that is linked to autophagosome formation [54]. Rab33 is a subclass within the Rab family consisting of the two homologous members Rab33A and Rab33B [150]. Rab33A is brain-specific [149] and Rab33B is ubiquitously expressed and localized at the medial Golgi cisternae [150]. Rab33 plays a role in intra-Golgi and Golgi-ER trafficking [127, 140]. Both Rab33B and to a lesser extent Rab33A interact with Atg16L1 in a GTP-dependent manner [54]. Atg16L is the mammalian orthologue of yeast Atg16.

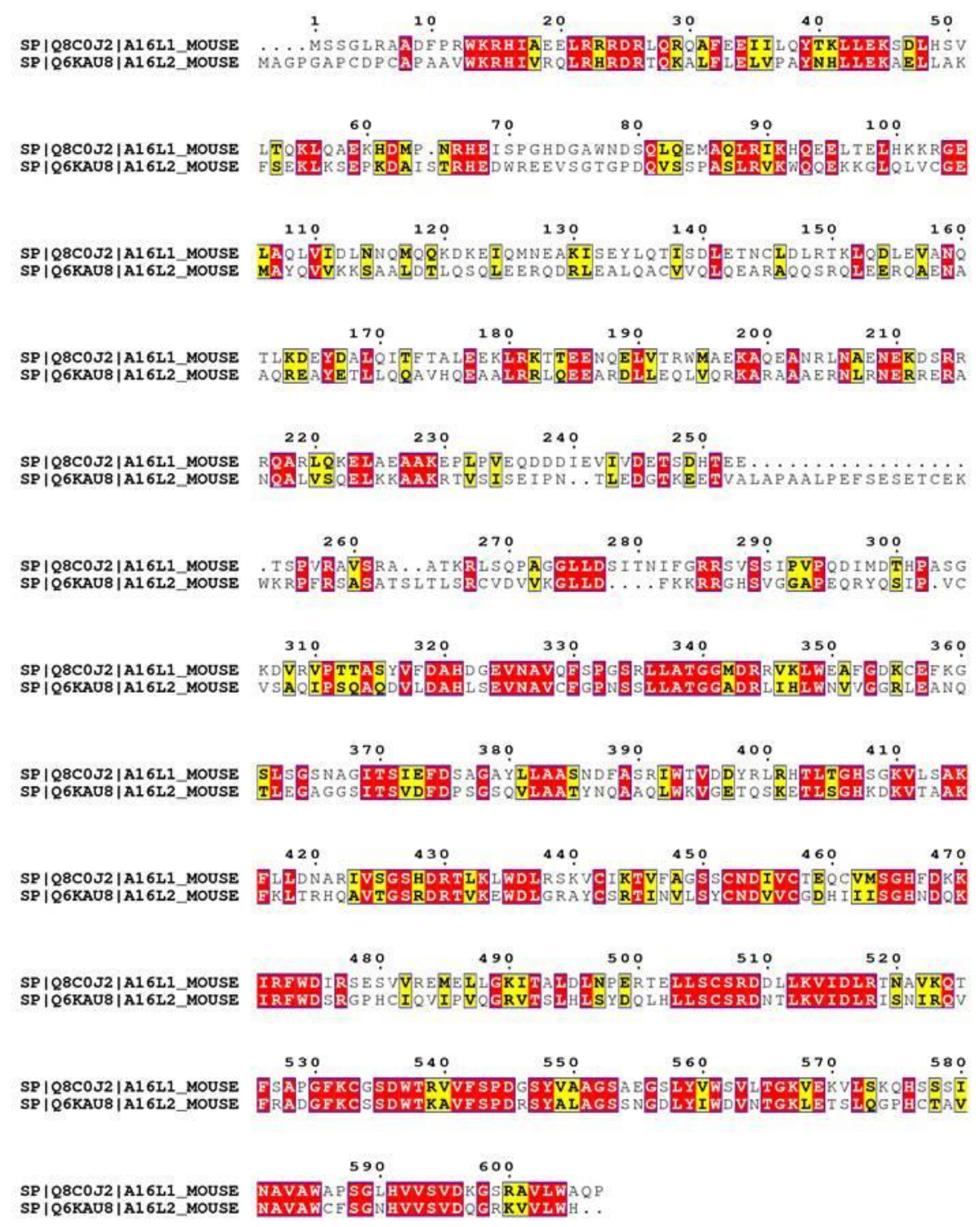

Figure 1.4.3.: Sequence alignment of murine Atg16L isoforms. Similar residues are colored black and yellow boxed, identical residues are colored white and red boxed. Alignment was done with Clustal W, Figure was made using ESPript 3.0 [116].

The yeast Atg5 Atg12/Atg16 complex has a size of $\sim 350 \mathrm{kDa}$ [75] and the mammalian complex has an estimated molecular weight of $\sim 800 \mathrm{kDa}$ [90]. Rab33B-Atg16L1 binding may 
be enhanced by Atg16-Atg5 complex formation [21]. Atg16 contains an N-terminal Atg5binding region, a coiled-coil domain and unlike yeast, Atg16L has an additional C-terminal WD40 domain that forms a $\beta$-propeller that has been suggested to mediate protein-protein interactions. Yeast Atg16 forms a parallel dimeric coiled-coil [37]. Similarly, Atg16L homodimerizes through its coiled coil domain [107]. The two mammalian isoforms Atg16L1 and Atg16L2 share a conserved Atg5-binding region and the WD40 domain but differ in the coiled-coil domain (Fig. 1.4.3.) [53]. Atg16L1 binding to Rab33B was located to the coiled coil domain residues 80-265 and not to the Atg5-binding site or the WD 40 domain (Fig. 1.4.4. A) [54]. Both isoforms share the biochemical properties of homo-dimerization and Atg5 Atg12/Atg16L complex formation. Similar to Atg16L1, the coiled coil domain of Atg16L2 only binds Rab33A/B among 58 tested Rab proteins [53]. However, compared to Atg16L1 the Rab33B binding affinity of Atg16L2 is approx.100 times lower (Fig. 1.4.4. B) [53]. Atg16L2 is not located to the phagophore and its physiological role remains unclear [53]. The Atg5 Atg12/Atg16L1 complex localizes mainly at the outward edges of the growing isolation and acts as a novel E3 ligase on Atg3 and determines the LC3 lipidation site [49].

A

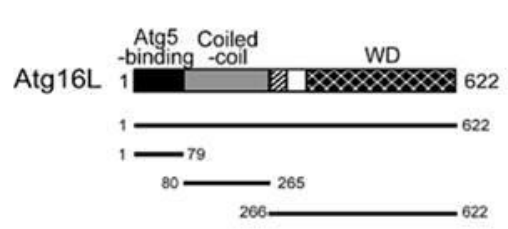

B

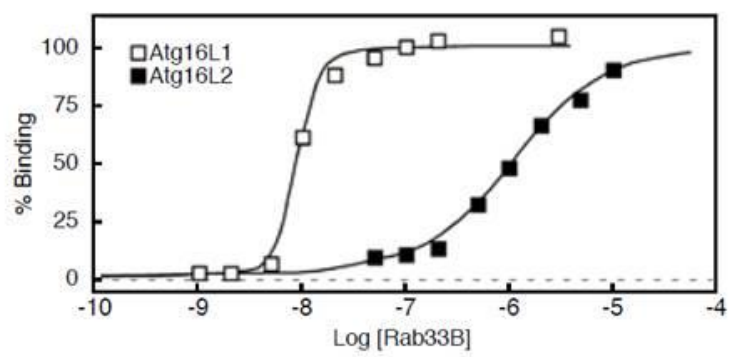

Figure 1.4.4. Rab33B binding of Atg16L. A: Schematic representation of Atg16L1 constructs tested for FLAG-Rab33B and FLAG-Atg5 co-immunoprecipitation. Figure A modified from [54]. B: Binding curve of Atg16L1 and Atg16L2 binding to increasing concentrations of Rab33B. Figure B from [53].

Rab33B-Atg16L1 interaction may facilitate the recruitment of vesicles originating from Golgi apparatus to the growing isolation membrane (Fig. 1.4.5.) [54]. As the same study did find an effect on autophagosome formation when Rab33B was silenced by siRNA, the role of Rab33B in autophagy needs to be further investigated. A recent study shows that the Hepatitis B virus exploits this interaction and Rab33B together with the Atg5 Atg12/Atg16L1 complex assist in naked capsid formation and secretion of the virus [30]. 


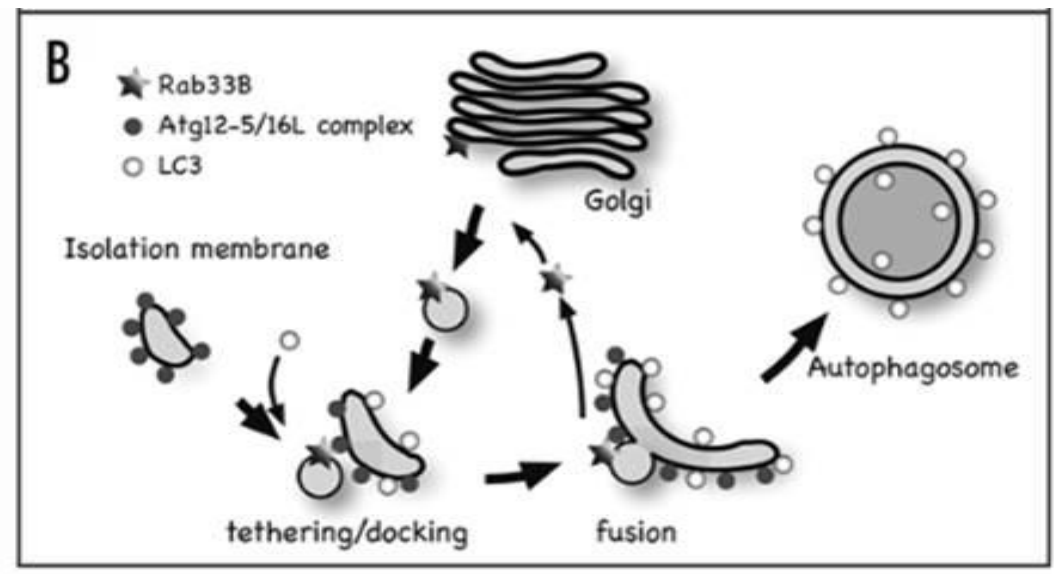

Figure 1.4.5.: Suggested role of Rab33B in autophagy. Rab33B-Atg16L1 binding delivers Golgi derived vesicles to the growing isolation membrane. Figure modified from [40].

\subsection{The role of the autophagy related SCOC-FEZ1 complex}

The short coiled coil protein (SCOC or SCOCO) was identified as a positive regulator of autophagy in a genome-wide siRNA screen [86]. SCOC, a Golgi-protein, is widely expressed in human tissue, most abundant in the brain, heart and skeletal muscles [141]. SCOC is an effector of Arl1 [141] and Arl3 [105] and colocalizes with Golgi-associated proteins TGN46 and mAtg9 [86], suggesting a function in Golgi transport. Four different isoforms produced by alternative splicing with a shared conserved coiled-coil domain were identified (Fig. 1.5.1.).
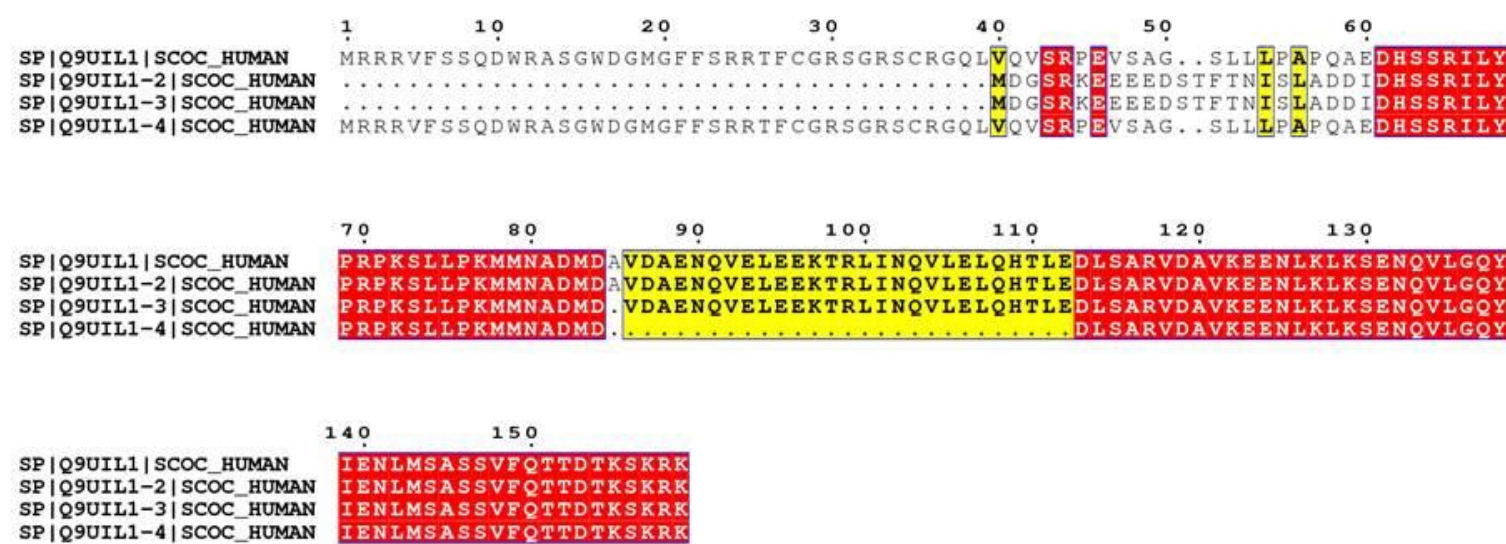

Figure 1.5.1.: Sequence alignment of SCOC isoforms. Similar residues are colored black and yellow boxed, identical residues are colored white and red boxed. Alignment was done with Clustal W, Figure was made using ESPript 3.0 [116]. 
SCOC forms a complex with fasciculation and elongation protein zeta 1 (FEZ1) [11]. FEZ1 is an adaptor for kinesin mediated axonal transport of the SNARE Syntaxin-1 and its function is regulated by phosphorylation [20, 25]. Human SCOC-FEZ1 complex formation is conserved because their orthologues in Caenorhabditis elegans interact as well [132]. UNC-69, the orthologue of SCOC interacts with UNC-76, a kinesin heavy chain adaptor and the orthologue of mammalian FEZ1 [15]. The UNC-69/UNC-76 complex is linked to axonal outgrowth and is required for normal presynaptic organization [132]. Besides its role in neuronal transport processes, the SCOC-FEZ1 complex is involved in the induction and regulation of autophagy upon starvation (Fig. 1.5.2.). FEZ1 interacts with the mammalian ULK1 kinase complex [57]. SCOC binding to FEZ1 disassembles the FEZ1-ULK1 complex and released ULK1 induces autophagy. SCOC-FEZ1 also forms a trimeric complex with UVRAG (UV radiation resistance associated gene) [86]. Starvation induces dissociation of the complex and UVRAG activates the autophagy promoting Vps34 kinase (Beclin 1-PI3K) complex. In vivo studies showed that siRNA knockdown of SCOC decreases LC3 lipidation, while overexpression increases LC3 lipidation upon starvation. Whereas FEZ1 knockdown increases and overexpression decreases LC3 lipidation [86]. Besides FEZ1 its paralog FEZ2 was identified [15]. Both proteins share the conserved coiled coil region [39]. Using a yeast two-hybrid system FEZ2 was shown to interact with the same interaction partners as FEZ1, like the interaction with SCOC, and 19 additional proteins [3]. However, until now no direct interaction between FEZ2 and SCOC was demonstrated. 


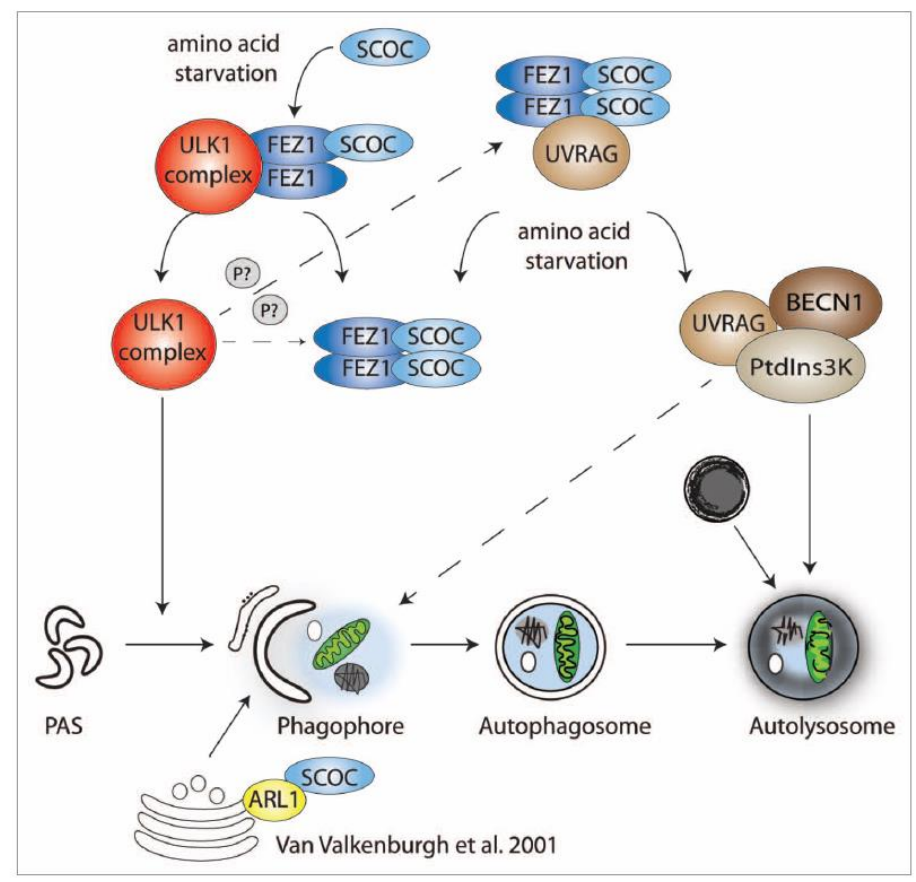

Figure 1.5.2.: Model for the possible SCOC-FEZ1 complex involvement in autophagy. Upon starvation SCOC binds to FEZ1 and releases the positive autophagy regulator ULK1. Under starvation conditions ULK1 may also regulate the release of UVRAG from a complex with SCOC-FEZ1. UVRAG binds to the Vsp34 kinase complex (BECN1 and PtdIns3K) that is part of the autophagy core machinery. Figure from [57].

The crystal structure of the SCOC coiled coil domain was published by our group [11]. SCOC forms a left handed parallel coiled coil dimer (Fig. 1.5.3.). Human FEZ1 is a mainly natively unfolded protein with a conserved coiled coil domain in the $\mathrm{C}$-terminal half of the protein (Fig. 1.5.4.) [76]. SCOC-FEZ1 complex formation is mediated through the coiled coil domains of SCOC and FEZ1. FEZ1 coiled coil residues L254 and L260 are important for SCOC-FEZ1 complex formation [86] as well as the corresponding residues in UNC-76 for UNC-69/UNC-76 complex formation (Fig. 1.5.4.) [132]. Additionally, the SCOC surface residue R117 is important for FEZ1 binding (Fig. 1.5.4.) [11]. The minimal regions required for complex formation are M78-K159 for SCOC and FEZ1 R227-K290 [11]. 

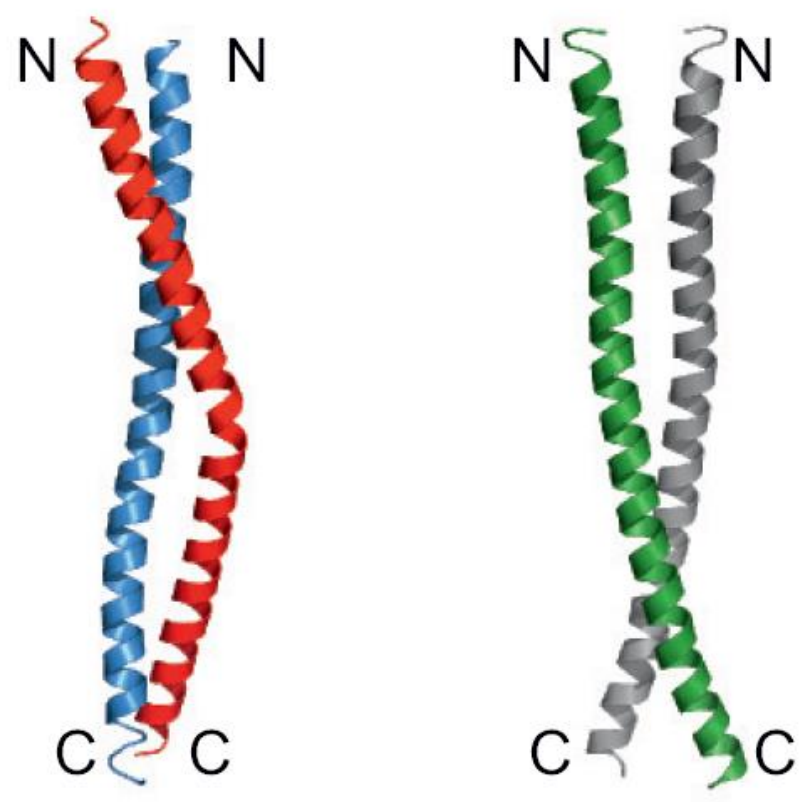

Figure 1.5.3.: Crystal structure of the SCOC coiled coil domain. Cartoon representation of the two dimers $\mathrm{AB}$ and $\mathrm{CC}^{\prime}$. Molecule $\mathrm{A}$ is colored red, $\mathrm{B}$ blue, $\mathrm{C}$ green and $\mathrm{C}^{\prime}$ grey. Adapted from [11]

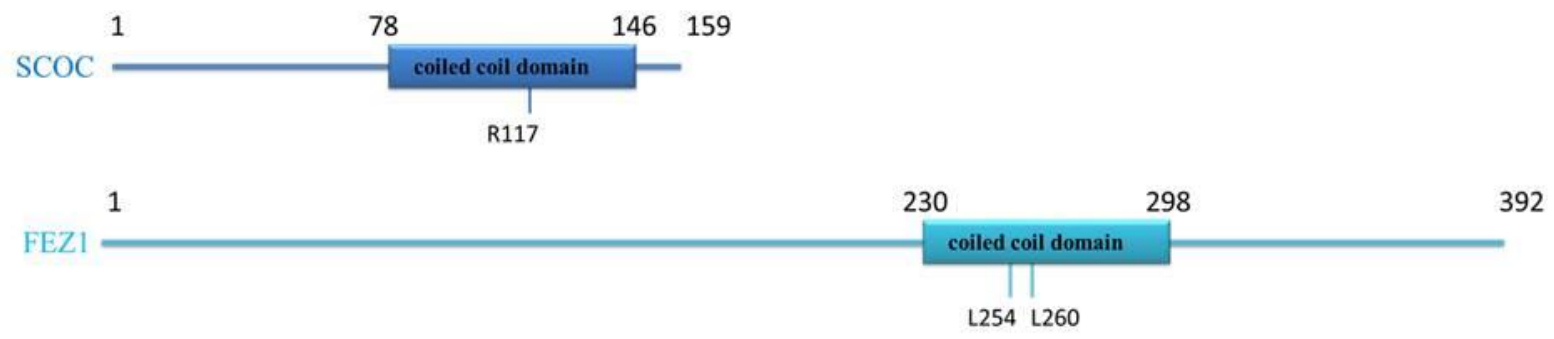

Figure 1.5.4.: Domaine structure of human SCOC and FEZ1. Residues below coiled coil domains were shown to be important for SCOC-FEZ1 complex formation. 


\section{Materials and Methods}

\subsection{Materials}

\subsubsection{Chemicals and media supplements}

Chemicals and media supplements used in this study are listed in table 2.1.1.

Table 2.1.1.: Chemicals used in this study

\begin{tabular}{|c|c|}
\hline Chemicals & Manufacturer \\
\hline 6x DNA loading dye & Thermo Scientific \\
\hline Agar & $\mathrm{BD}$ \\
\hline Amino acids & Sigma-Aldrich \\
\hline Ammonium persulfate (APS) & Sigma-Aldrich \\
\hline complete EDTA-free, Protease inhibitor tablet & Roche \\
\hline Coomassie R Brilliant blue R250 & Fluka \\
\hline D-Glucose monohydrate & Merck \\
\hline D-Lactose & Fluka \\
\hline DMEM & Lonza \\
\hline DMEM/F-12 & Sigma Aldrich \\
\hline FCS & PAA laboratories \\
\hline GelGreen & Biotium \\
\hline GeneRuler $^{\mathrm{TM}} 1 \mathrm{~kb}$ DNA Ladder & Thermo Scientific \\
\hline GeneRuler $^{\mathrm{TM}} 100$ bp DNA Ladder & Thermo Scientific \\
\hline GTP $\gamma \mathrm{S}$ & Jena Bioscience \\
\hline HEPES ultrapure & GERBU Biotech \\
\hline Imidazole & Sigma-Aldrich \\
\hline IPTG, Dioxane free & ForMedium \\
\hline Lipofectamine $^{\mathrm{TM}} 2000$ & Thermo Scientific \\
\hline $\mathrm{NaF}$ & Sigma-Aldrich \\
\hline Ni-NTA Sepharose & GE Healthcare \\
\hline N-Z-AmineAS & Sigma-Aldrich \\
\hline Odyssey® One-Color Protein Molecular Weight Marker & LI-COR \\
\hline PageRuler ${ }^{\mathrm{TM}}$ Prestained Protein Ladder & Thermo Scientific \\
\hline Rotiphorese ${ }^{\circledR G e l} 30$ (37.5:1) & Roth \\
\hline Selenomethionine & Tokyo Chemical Industry \\
\hline Spectra $^{\mathrm{TM}}$ Multicolor Low Ranged Protein Ladder & Thermo Scientific \\
\hline Tris(2-carboxyethyl)phosphine & Sigma-Aldrich \\
\hline Tris-(hydroxymethyl)aminomethane & VWR chemicals \\
\hline Triton-X-100 & Sigma-Aldrich \\
\hline Tryptone/Peptone from Casein & Roth \\
\hline Ultra-low Range Molecular Weight Marker & Sigma-Aldrich \\
\hline Western Lightning® Plus-ECL & PerkinElmer \\
\hline Yeast extract & Roth \\
\hline Yeast extract-B & QBiogene \\
\hline$\beta$-mercaptoethanol & Sigma-Aldrich \\
\hline
\end{tabular}




\subsubsection{Buffers, media and antibiotics}

Buffers, media and antibiotics used in this study are listed in tables 2.1.2. to 2.1.4.

Table 2.1.2.: Buffers used in this study

\begin{tabular}{|c|c|}
\hline Buffer & Content \\
\hline $\begin{array}{l}\text { Anode buffer for Schägger } \\
\text { gel electrophoresis }\end{array}$ & $0.2 \mathrm{M}$ Tris to $\mathrm{pH} 8.9$ with $\mathrm{HCl}$ \\
\hline $\begin{array}{l}\text { Cathode Buffer Schägger gel } \\
\text { electrophoresis }\end{array}$ & $0.1 \mathrm{M}$ Tris, $0.1 \mathrm{M}$ Tricine \\
\hline CD buffer & $20 \mathrm{mM}$ sodium phosphate $\mathrm{pH} 7.5,150 \mathrm{mM} \mathrm{NaF}$ \\
\hline $\begin{array}{l}\text { Coomassie Blue staining } \\
\text { solutions }\end{array}$ & $\begin{array}{l}\text { A: } 500 \mathrm{mg} \text { Coomassie }, 650 \mathrm{ml} \mathrm{H}_{2} \mathrm{O}, 250 \mathrm{ml} \text { isopropanol, } 100 \mathrm{ml} \\
\text { acetic acid }\end{array}$ \\
\hline & 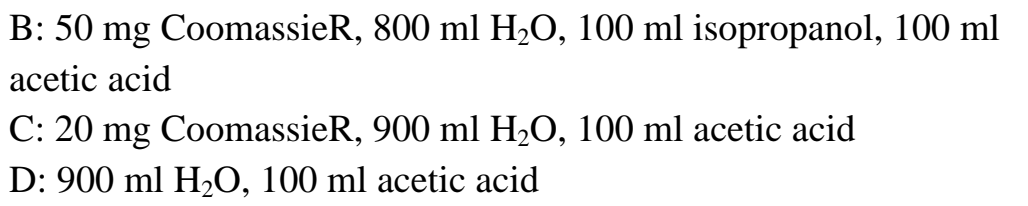 \\
\hline $\begin{array}{l}\text { Gel buffer for Schägger gel } \\
\text { electrophoresis }\end{array}$ & $3 \mathrm{M}$ Tris $\mathrm{pH} 8.45,0.3 \% \mathrm{SDS}$ \\
\hline PBS & $150 \mathrm{mM} \mathrm{NaCl}, 20 \mathrm{mM} \mathrm{NaH} 2 \mathrm{PO} 4 \mathrm{pH} 7.4$ \\
\hline PBST & $150 \mathrm{mM} \mathrm{NaCl}, 20 \mathrm{mM} \mathrm{NaH} 2 \mathrm{PO} 4 \mathrm{pH}$ 7.4, 0,05\% (w/v) Tween 20 \\
\hline $\begin{array}{l}\text { Sample buffer for Schägger } \\
\text { gel electrophoresis }\end{array}$ & $\begin{array}{l}15 \mathrm{ml} 0.5 \mathrm{M} \text { Tris } \mathrm{pH} 6.8,6 \mathrm{~g} \text { SDS, } 15 \mathrm{mg} \text { Serva Blue, } 4.33 \mathrm{~g} \\
\text { Glycerol, } \mathrm{H}_{2} \mathrm{O} \text { to } 50 \mathrm{ml} \\
\text { make } 10 \mathrm{ml} \text { aliquots, add } 200 \mu \mathrm{l} \beta \text {-Mercaptoethanol }\end{array}$ \\
\hline TAE & $40 \mathrm{mM}$ Tris, $20 \mathrm{mM}$ Acetate, $1 \mathrm{mM}$ EDTA \\
\hline Western blot transfer buffer & $\begin{array}{l}25 \mathrm{mM} \text { Tris, } 192 \mathrm{mM} \text { glycine, } 0.04 \%(\mathrm{w} / \mathrm{v}) \mathrm{SDS}, 20 \%(\mathrm{v} / \mathrm{v}) \\
\text { methanol }\end{array}$ \\
\hline
\end{tabular}

Table 2.1.3.: Media used in this study

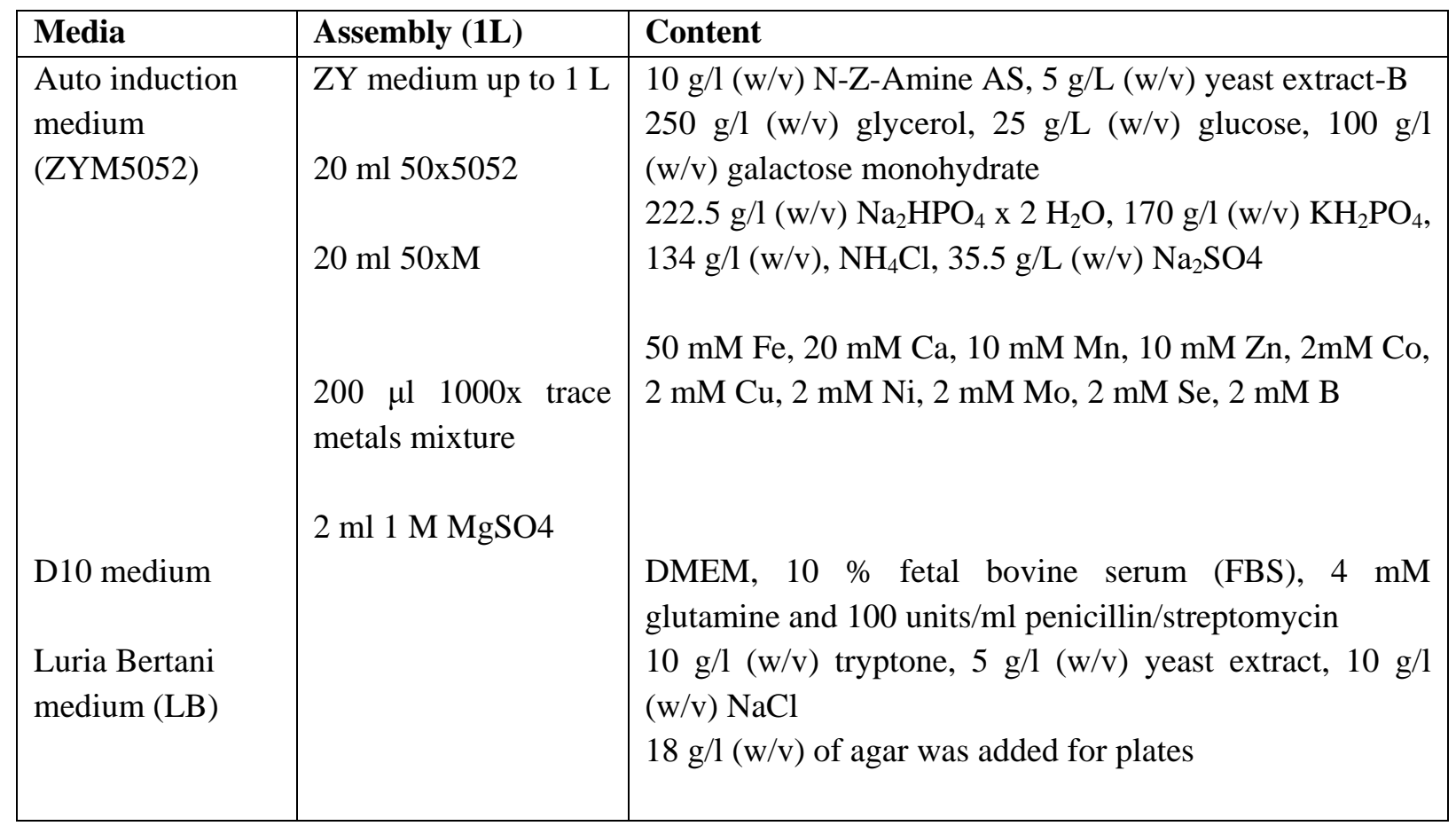




\begin{tabular}{|c|c|c|}
\hline Minimal medium & $\begin{array}{l}\text { M9 medium up to } \\
1 \mathrm{~L}\end{array}$ & $\begin{array}{l}6 \mathrm{~g} / \mathrm{l}(\mathrm{w} / \mathrm{v}) \mathrm{Na}_{2} \mathrm{HPO}_{4}, 3 \mathrm{~g} / \mathrm{l}(\mathrm{w} / \mathrm{v}) \mathrm{KH}_{2} \mathrm{PO}_{4}, 1 \mathrm{~g} / \mathrm{l}(\mathrm{w} / \mathrm{v}) \\
\mathrm{NH}_{4} \mathrm{Cl}, 0.5 \mathrm{~g} / \mathrm{l}(\mathrm{w} / \mathrm{v}) \mathrm{NaCl}, 4 \mathrm{~g} / \mathrm{l}(\mathrm{w} / \mathrm{v}) \text { glucose, } 1 \mathrm{mM} \\
\mathrm{MgSO}_{4}, 4.2 \mathrm{mg} / \mathrm{l} \mathrm{FeIISO}, 4,5 \mathrm{mg} / \mathrm{ml} \text { thiamine vitamin } \\
100 \quad \mathrm{mg} / \mathrm{l} \quad(\mathrm{w} / \mathrm{v}) \quad \mathrm{L}-\mathrm{Lysine}, 100 \quad \mathrm{mg} / \mathrm{l} \quad(\mathrm{w} / \mathrm{v}) \quad \mathrm{L}- \\
\text { Phenylalanine, } 100 \mathrm{mg} / \mathrm{l} \quad(\mathrm{w} / \mathrm{v}) \text { L-Threonine, } 50 \mathrm{mg} / \mathrm{l} \\
(\mathrm{w} / \mathrm{v}) \text { L-Isoleucine, } 50 \mathrm{mg} / \mathrm{l}(\mathrm{w} / \mathrm{v}) \text { L-Leucine, } 50 \mathrm{mg} / \mathrm{l} \\
(\mathrm{w} / \mathrm{v}) \text { L-Valine }\end{array}$ \\
\hline SOC medium & Selenomethionine & $\begin{array}{l}50 \mathrm{mg} / \mathrm{l}(\mathrm{w} / \mathrm{v}) \mathrm{L}-\mathrm{Selenomethionine} \\
2 \%(\mathrm{w} / \mathrm{v}) \text { tryptone, } 0.5 \%(\mathrm{w} / \mathrm{v}) \text { yeast extract, } 10 \mathrm{mM} \\
\mathrm{NaCl}, 2.5 \mathrm{mM} \mathrm{KCl} \\
\text { after autoclaving add } 10 \mathrm{mM} \mathrm{MgCl}_{2}, 10 \mathrm{mM} \mathrm{Mg}_{2} \mathrm{SO} 4,20 \\
\mathrm{mM} \text { glucose }\end{array}$ \\
\hline $\begin{array}{l}\text { Terrific broth } \\
\text { medium (TB) }\end{array}$ & & 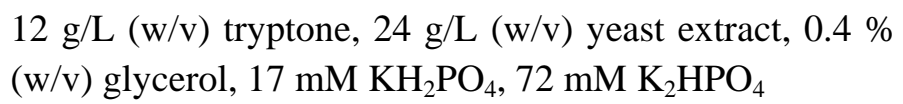 \\
\hline
\end{tabular}

Table 2.1.4.: Antibiotics used in this study

\begin{tabular}{|l|l|l|}
\hline Antibiotic & Concentration & Manufacturer \\
\hline Ampicillin & $100 \mu \mathrm{g} / \mathrm{ml}(\mathrm{w} / \mathrm{v})$ & Sigma-Aldrich \\
Kanamycin & $30 \mu \mathrm{g} / \mathrm{ml}(\mathrm{w} / \mathrm{v})$ & Sigma-Aldrich \\
\hline
\end{tabular}

Antibiotics were prepared as 1000x stock solutions in deionized water. All solutions were filter sterilized and stored at $-20{ }^{\circ} \mathrm{C}$.

\subsubsection{Enzymes}

Enzymes used in this study are listed in table 2.1.5.

Table 2.1.5.: Enzymes used in this study

\begin{tabular}{|l|l|}
\hline Enzyme & Manufacturer \\
\hline Alkaline Phosphatase & NEB \\
DNaseI & AppliChem \\
Lysozyme & Roth \\
Restriction enzymes & NEB \\
Substilisin & Hampton Research \\
T4 DNA Ligase & NEB \\
Thrombin & MP Biomedical \\
\hline
\end{tabular}

Thrombin was prepared in $10 \mathrm{mM}$ Tris $\mathrm{pH} 7.4,1 \mathrm{mM}$ EDTA and $50 \%$ glycerol.

\subsubsection{Kits}

Kits used in this study and suppliers are listed in table 2.1.6. 
Table 2.1.6.: Kits used in this study

\begin{tabular}{|c|c|}
\hline Name & Manufacturer \\
\hline NucleoSpin Plasmid kit & Macherey \& Nagel \\
\hline NucleoSpin Extract II kit & Macherey \& Nagel \\
\hline NucleoBond Xtra & Macherey \& Nagel \\
\hline Phusion High-Fidelity PCR Kit & NEB \\
\hline Gibson Assembly® & NEB \\
\hline QuickChange Lightning Site-Directed Mutagenesis Kit & Agilent Technologies \\
\hline $\mathrm{pENTR}^{\mathrm{TM}} / \mathrm{D}-\mathrm{TOPO}{ }^{\circledR}$ Cloning Kit & Thermo Scientific \\
\hline Western Lightening Plus-ECL & Perkin Elmer \\
\hline Additive Screen $^{\mathrm{TM}}$ & Hampton Research \\
\hline Additive Screen ${ }^{\mathrm{TM}}-\mathrm{HT}$ & Hampton Research \\
\hline Silver Bullets ${ }^{\mathrm{TM}}$ & Hampton Research \\
\hline CryoProt $X^{\mathrm{TM}}$ & Molecular Dimensions \\
\hline JBS Magic Triangle & Jena Bioscience \\
\hline
\end{tabular}

\subsubsection{Columns for chromatography}

Columns for chromatography used in this study are listed in table 2.1.7. All columns were used in combination with an Äkta Purifier FPLC system (RT) or with the Äkta Prime FPLC $\left(4{ }^{\circ} \mathrm{C}\right)$. The manufacturer's recommendations were followed for handling of the columns, including storage, cleaning and equilibration.

Table 2.1.7.: Columns for chromatography used in this study

\begin{tabular}{|l|l|}
\hline Column & Manufacturer \\
\hline $5 \mathrm{ml}$ HisTrap FF column & GE Healthcare \\
$5 \mathrm{ml}$ Strep-Trap column & GE Healthcare \\
HiLoad 16/60 Superdex 200 prep grade & GE Healthcare \\
HiLoad 16/60 Superdex 75 prep grade & GE Healthcare \\
Superdex 200 10/300 GL & GE Healthcare \\
\hline
\end{tabular}

\subsubsection{Antibodies}

Antibodies used in this study are listed in table 2.1.8.

Table 2.1.8.: Antibodies used in this study

\begin{tabular}{|l|l|l|l|}
\hline Primary Antibody & Clonality & Host & Manufacturer \\
\hline Anti-Atg16L pAb (PM040) & Polyclonal & Rabbit & MBL Life Science \\
GFP antibody (132002) & Polyclonal & Rabbit & Synaptic Systems \\
Hexa-Histidine tag (DIA900) & Monoclonal & Mouse & Dianova \\
LC3B Antibody (NB100-2220) & Polyclonal & Rabbit & Novus Biologicals \\
Strep-tag II antibody (ABIN3197820) & Monoclonal & Mouse & IBA GmbH \\
Penta His HRP conjugate (34460) & Monoclonal & Mouse & Qiagen GmbH \\
V5 tag antibody - ChIP Grade (ab9116) & Polyclonal & Rabbit & Abcam \\
\hline
\end{tabular}




\begin{tabular}{|l|l|l|l|}
\hline V5-Probe (sv5-pk) Antibody (sc-58052) & Monoclonal & Mouse & Santa Cruz Biotechnology \\
WIPI2 antibody (ab131271) & Monoclonal & Rabbit & Abcam \\
\hline Secondary Antibody & Clonality & Host & Manufacturer \\
\hline mouse IgG IR dye 800 & Polyclonal & Goat & LI-COR \\
mouse IgG (HRP labeled) & Polyclonal & Goat & BioRad Laboratories \\
mouse IgG light chain specific (HRP & Polyclonal & Goat & BioRad Laboratories \\
labeled) & & & \\
mouse IgG Cy3 & Polyclonal & Goat & Jackson ImmunoResearch \\
rabbit IgG IR dye 800 & Polyclonal & Goat & LI-COR \\
rabbit IgG (HRP labeled) & Polyclonal & Goat & BioRad Laboratories \\
\hline
\end{tabular}

\subsubsection{Bacterial strains}

Bacterial strains used in this study are listed in table 2.1.9.

Table 2.1.9.: Bacterial strains used in this study

\begin{tabular}{|l|l|l|}
\hline Strain & Manufacturer & Usage \\
\hline E. coli XL1-blue & Agilent Technologies & Standard cloning \\
E. coli XL10-Gold ultracompetent & Agilent Technologies & Mutagenesis cloning \\
E. coli NEB ${ }^{\circledR}$ 5-alpha competent & NEB $\AA$ & GIBSON Assembly cloning \\
(High Efficiency) & & \\
E. coli BL21-Gold(DE3) competent & Agilent Technologies & Expression \\
E. coli Rosetta $^{\mathrm{TM}}$ 2(DE3)pLysS & Novagen & Expression \\
\hline
\end{tabular}

\subsubsection{DNA constructs}

DNA constructs produced and/or used in this study are listed in tables 2.1.10. to 2.1.12.

Table 2.1.10.: DNA constructs in single cloning site vectors for bacterial expression. Abbreviation Restr. Sites: Restriction sites

\begin{tabular}{|l|l|l|l|l|l|}
\hline Gene & Vector & Tag & Restr. sites & Resistance & Source \\
\hline mAtg16L1 (153-210) & pET-28a & $6 x$ His & NdeI / XhoI & Kanamycin & J.Groffmann \\
KlAtg21 & pET-28a & $6 x$ His & NdeI / XhoI & Kanamycin & K. Kühnel \\
KlAtg21 R103E & pET-28a & $6 x$ His & NdeI / XhoI & Kanamycin & J. Metje \\
AgAtg16 (40-124) & pET-28a & $6 x$ His & NdeI / XhoI & Kanamycin & K. Kühnel \\
AgAtg16 (70-124) & pET-28a & $6 x$ His & NdeI / XhoI & Kanamycin & K. Kühnel \\
AgAtg16 (70-124) D78R & pET-28a & 6x His & NdeI / XhoI & Kanamycin & J. Metje \\
SCOC (78-159) & pET-28a & Strep II & NcoI / XhoI & Kanamycin & C. Behrens \\
SCOC (78-159) L105M & pET-28a & Strep II & NcoI / XhoI & Kanamycin & C. Behrens \\
FEZ1 (227-290) & pET-22b & 6x His & NdeI / XhoI & Ampicillin & C. Behrens \\
FEZ1 (225-295) & pET-22b & 6x His & NdeI / XhoI & Ampicillin & C. Behrens \\
FEZ1 (225-295) L251M & pET-22b & 6x His & NdeI / XhoI & Ampicillin & J. Metje \\
FEZ1 (225-295) L273M & pET-22b & 6x His & NdeI / XhoI & Ampicillin & J. Metje \\
\hline
\end{tabular}


Table 2.1.11.: DNA constructs in pET-Duet1 vector for bacterial expression. All constructs were cloned with restriction sites BamHI / NotI for MCSI and NdeI / XhoI for MCS II.

\begin{tabular}{|c|c|c|c|c|c|}
\hline MCS I & Tag & MCS II & Tag & Resistance & Source \\
\hline mRab33B cDNA (1-229) & His & - & - & Ampicillin & A. Scacioc \\
\hline ВC065076 & & & & & \\
\hline mRab33B (30-202) Q92L & 6x His & - & - & Ampicillin & K. Kühnel \\
\hline mRab33B (30-202) Q92L & 6x His & mAtg16L1 (125-234) & - & Ampicillin & M.Hellwig \\
\hline mRab33B (30-202) Q92L & 6x His & mAtg16L1 (127-210) & - & Ampicillin & K. Kühnel \\
\hline mRab33B (30-202) Q92L & 6x His & mAtg16L1 (153-200) & - & Ampicillin & K. Kühnel \\
\hline mRab33B (30-202) Q92L & 6x His & mAtg16L1 (153-210) & - & Ampicillin & K. Kühnel \\
\hline mRab33B (30-202) Q92L & 6x His & mAtg16L1 (163-210) & - & Ampicillin & K. Kühnel \\
\hline mRab33B (30-202) & 6x His & mAtg16L1 (153-210) & - & Ampicillin & J. Metje \\
\hline mRab33B (30-202) T47N & 6x His & mAtg16L1 (153-210) & - & Ampicillin & J. Metje \\
\hline $\begin{array}{l}\text { mRab33B (30-202) Q92L, } \\
\text { F70E }\end{array}$ & 6x His & mAtg16L1 (153-210) & - & Ampicillin & J. Metje \\
\hline $\begin{array}{l}\text { mRab33B (30-202) Q92L, } \\
\text { F70A }\end{array}$ & 6x His & mAtg16L1 (153-210) & - & Ampicillin & J. Metje \\
\hline $\begin{array}{l}\text { mRab33B (30-202) Q92L, } \\
\text { W87A }\end{array}$ & 6x His & mAtg16L1 (153-210) & - & Ampicillin & J. Metje \\
\hline mRab33B (30-202) Q92L & 6x His & $\begin{array}{l}\text { mAtg16L1 (153-210) } \\
\text { K198A }\end{array}$ & - & Ampicillin & J. Metje \\
\hline mRab33B (30-202) Q92L & 6x His & $\begin{array}{l}\text { mAtg16L1 (153-210) } \\
\text { A202W }\end{array}$ & - & Ampicillin & J. Metje \\
\hline mRab33B (30-202) Q92L & 6x His & $\begin{array}{l}\text { mAtg16L1 (153-210) } \\
\text { N206K }\end{array}$ & - & Ampicillin & J. Metje \\
\hline
\end{tabular}

Table 2.1.12.: DNA constructs in Gateway vectors for mammalian expression

\begin{tabular}{|c|c|c|c|c|}
\hline Gene & Vector & Tag & Resistance & Source \\
\hline mRab33B c & ET-Duet1 & His & Ampicillin & A. Scacioc \\
\hline mRab33B (1-229) WT & pENTR ${ }^{\mathrm{TM}} / \mathrm{D}-\mathrm{TOPO}{ }^{\circledR}$ & 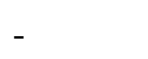 & Ampicillin & J. Metje \\
\hline mRab33B (1-229) WT & pcDNA3.1/nV5 & V5 & Ampicillin & J. Metje \\
\hline mRab33B (1-229) Q92L & pcDNA3.1/nV5 & V5 & Ampicillin & J. Metje \\
\hline mRab33B (1-229) T47N & pcDNA3.1/nV5 & V5 & cillin & J. Metje \\
\hline mRab33B (1-229) Q92L F70A & pcDNA3.1/nV5 & V5 & Ampicillin & J. Metje \\
\hline mRab33B (1-229) Q92L F70E & pcDNA3.1/nV5 & V5 & Ampicillin & J. Metje \\
\hline mRab33B (1-229) Q92L W87A & pcDNA3.1/nV5 & V5 & Ampicillin & J. Metje \\
\hline mAtg16L1 (1-632) WT & pcDNA6.1/C-EmGFP & EmGFP & Ampicillin & A. Scacioc \\
\hline mAtg16L1 (1-632) K198A & pcDNA6.1/C-EmGFP & EmGFP & Ampicillin & J. Metje \\
\hline A202W & pcDNA6.1/C-EmGFP & EmGFP & illin & J. Metje \\
\hline mAtg16L1 (1-632) N206K & pcDNA6.1/C-EmGFP & EmGFP & Ampicillin & J. Metje \\
\hline
\end{tabular}

\subsubsection{Oligonucleotides}

Oligonucleotides used in this study are listed in table 2.1.13. Oligonucleotides were ordered from Eurofins Genomics GmbH 
Table 2.1.13.: Oligonucleotides strains used in this study

\begin{tabular}{|c|c|c|}
\hline Name & Usage & Sequence 5 to 3 \\
\hline mRab33Bfl_TOPO_fw & Gateway Cloning & caccacttcggagatggagtcgtc \\
\hline mRab33Bfl_TOPO_rev & Gateway Cloning & tcagcaccagcaagtcacc \\
\hline mRab33B_L92Q_fw & Mutagenesis & ctgaaccgctcctgccetgccgtgt \\
\hline mRab33B_L92Q_rev & Mutagenesis & acacggcagggcaggagcggttcag \\
\hline mRab33B_Q92L_fw & Mutagenesis & ctgaaccgctccagecctgccgtgt \\
\hline mRab33B_Q92L_rev & Mutagenesis & acacggcagggctggagcggttcag \\
\hline mRab33B_T47N_fw & Mutagenesis & tcgaacgtgggcaagaactgcctgacttaccgc \\
\hline mRab33B_T47N_rev & Mutagenesis & gcggtaagtcaggcagttcttgcccacgttcga \\
\hline mRab33B_F70E_fw & Mutagenesis & cacggetcgetctcgttcgtccaccecgatcgt \\
\hline mRab33B_F70E_rev & Mutagenesis & acgatcggggtggacgaacgagagcgagccgtg \\
\hline mRab33B_F70A_fw & Mutagenesis & cggctcgctctcgggegtccacccegatc \\
\hline mRab33B_F70A_rev & Mutagenesis & gatcggggtggacgcccgagagcgagccg \\
\hline mRab33B_W87A_fw & Mutagenesis & cagccetgccgtgtccgccaactggatcttgat \\
\hline mRab33B_W87A_rev & Mutagenesis & atcaagatccagttggcggacacggcagggctg \\
\hline mAtg16L1_153_GA_fw & $\begin{array}{l}\text { Gibson Assembly } \\
\text { Cloning }\end{array}$ & gccgcgcggcagccatatgcaggacctcgaggtagcc \\
\hline mAtg16L1_210_GA_rev & $\begin{array}{l}\text { Gibson Assembly } \\
\text { Cloning }\end{array}$ & cagtggtggtggtggtggtgctcgagctcattctctgcattgagg \\
\hline mAtg16 L1_K198E_fw & Mutagenesis & ttggcttcttgggectcctcagecatccatc \\
\hline mAtg16 L1_K198E_rev & Mutagenesis & gatggatggetgaggaggcccaagaagccaa \\
\hline mAtg16 L1_A202W_fw & Mutagenesis & ctctgcattgaggcgattccattcttgggecttctcagc \\
\hline mAtg16 L1_A202W_re & Mutagenesis & gctgagaaggcccaagaatggaatcgcctcaatgcagag \\
\hline mAtg16 L1_N206K_fw & Mutagenesis & accetcattctctgctttgaggcgattggcttc \\
\hline mAtg16 L1_N206K_fw & Mutagenesis & gaagccaatcgcctcaaagcagagaatgagggt \\
\hline AgAtg16_I81M_fw & Mutagenesis & cgatgttcagggacatgatctcgtcattcagtttctct \\
\hline AgAtg16_I81M_rev & Mutagenesis & agagaaactgaatgacgagatcatgtccctgaacatcg \\
\hline AgAtg16_D78R_fw & Mutagenesis & gggaaatgatctcgcgattcagtttctctgcgtctttactcat \\
\hline AgAtg16_D78R_rev & Mutagenesis & atgagtaaagacgcagagaaactgaatcgcgagatcatttccc \\
\hline K1Atg21_R103E_fw & Mutagenesis & tttcagcaccacacagatgatttttcgttcataatcacgtccatgatctc \\
\hline K1Atg21_R103E_re & Mutagenesis & gagatcatggacgtgattatgaacgaaaaaatcatctgtgtggtgctgaaa \\
\hline Fez1_L251M_fwd & Mutagenesis & tggtgcagcagatggccegccgg \\
\hline Fez1_L251M_rev & Mutagenesis & ccggcgggccatctgctgcacca \\
\hline Fez1_L273M_fwd & Mutagenesis & aagaactcctttatcacggtgatgattgaggttcagaacaagcag \\
\hline Fez1_L273M_rev & Mutagenesis & ctgcttgttctgaacctcaatcatcaccgtgataaaggagttctt \\
\hline M13_fwd & Sequencing & cgccagggttttcccagtcacgac \\
\hline M13_rev & Sequencing & tcacacaggaaacagctatgac \\
\hline T7_promoter & Sequencing & taatacgactcactataggg \\
\hline T7_terminator & Sequencing & ctagttattgctcagcggtg \\
\hline
\end{tabular}




\subsection{Methods}

\subsubsection{Molecular Cloning}

\subsubsection{Standard Cloning}

For standard cloning inserts were duplicated by PCR, cut from an agarose gel and purified. Inserts and vectors were restriction digested and ligated with $\mathrm{T} 7$ polymerase prior to transformation.

\section{PCR}

For PCR Phusion High-Fidelity PCR kit and primers flanking the insert were used. All reaction components (see Table 2.2.1.) were assembled on ice, mixed and collected to the bottom of the tube by a quick spin prior to PCR.

Table 2.2.1.: Standard PCR reaction assembly

\begin{tabular}{|l|l|}
\hline Component & Volume \\
\hline $\mathrm{H}_{2} \mathrm{O}$ & To $50 \mu \mathrm{l}$ \\
$5 \times$ Phusion HF Buffer & $10 \mu \mathrm{l}$ \\
$10 \mathrm{mM}$ dNTPS & $1 \mu \mathrm{l}$ \\
$10 \mu \mathrm{M}$ forward primer & $2,5 \mu \mathrm{l}$ \\
$10 \mu \mathrm{M}$ reverse primer & $2,5 \mu \mathrm{l}$ \\
Template DNA & Variable (50-100 ng) \\
Phusion DNA Polymerase & $0.5 \mu \mathrm{l}$ \\
\hline
\end{tabular}

PCRs were done in a Biometra T-Personal Combi Thermocycler and comprised the following steps (Table 2.2.2.). For standard procedure, steps 2-4 were repeated for 35 cycles.

Table 2.2.2.: Standard PCR reaction steps

\begin{tabular}{|l|l|l|}
\hline Step & Temperature & Time \\
\hline 1. Initial denaturation & $95{ }^{\circ} \mathrm{C}$ & $30 \mathrm{sec}$ \\
2. Denaturation & $95{ }^{\circ} \mathrm{C}$ & $10 \mathrm{sec}$ \\
3. Annealing & According to NEB Tm calculator & $20 \mathrm{sec}$ \\
4. Elongation & $72{ }^{\circ} \mathrm{C}$ & $15 \mathrm{sec}$ per kb \\
5. Final extension & $72{ }^{\circ} \mathrm{C}$ & $5 \mathrm{~min}$ \\
6. Hold & $12{ }^{\circ} \mathrm{C}$ & \\
\hline
\end{tabular}

\section{DNA agarose gel electrophoresis and gel extraction purification}

PCR products and DNA plasmids were visualized by 0.7-1 \% agarose gel electrophoresis. Samples for electrophoresis were mixed with 6 x loading dye. TAE buffer was used as 
running buffer. Agarose gels were stained with GelGreen ${ }^{\mathrm{TM}}$ Nucleic Acid Gel Stain and sizes of DNA products were estimated with DNA ladders. Gel extraction was done with the NucleoSpin Extract II kit according to the manufacturer's instructions.

\section{Restriction digestion}

Restriction digest reactions were carried out for one hour at $37{ }^{\circ} \mathrm{C}$ with restriction enzymes in $40 \mu 1$ reactions and heat inactivated when needed according to the manufacturer's instructions (see Table 2.2.3.).

Table 2.2.3.: Restriction digestion reaction assembly

\begin{tabular}{|l|l|}
\hline Component & Volume \\
\hline $\mathrm{H}_{2} \mathrm{O}$ & Up to $40 \mu \mathrm{l}$ \\
PCR product or vector & Variable (up to $1 \mu \mathrm{g}$ ) \\
10x CutSmart $®$ Buffer & $4 \mu \mathrm{l}$ \\
Enzyme 1 & $1 \mu \mathrm{l}$ \\
Enzyme 2 (if double digestion) & $1 \mu \mathrm{l}$ \\
BSA (if required) & $0,4 \mu \mathrm{l}$ \\
\hline
\end{tabular}

\section{Ligation}

Ligations were performed with 50-100 ng of restriction digested vector combined with restriction digested insert in a ratio of 1:3. A final reaction volume of $10 \mu 1$ was set up with $1 \mu \mathrm{l}$ of T4 DNA Ligase and $1 \mu \mathrm{l}$ of $10 \mathrm{x}$ buffer. Ligation was conducted at room temperature for $60 \mathrm{~min}$.

\subsubsection{Gibson Assembly ${ }^{\circledR}$ Cloning and Gateway ${ }^{\circledR}$ Cloning}

Gibson Assembly ${ }^{\circledR}$ Cloning was performed according to the manufacturer's instructions using the Gibson Assembly Kit from NEB.

\subsubsection{Gateway® Cloning}

Gibson Assembly ${ }^{\circledR}$ Cloning was performed according to the manufacturer's instructions using the pENTR ${ }^{\mathrm{TM}} / \mathrm{D}-\mathrm{TOPO}{ }^{\circledR}$ Cloning Kit and the Gateway ${ }^{\mathrm{TM}}$ LR Clonase ${ }^{\mathrm{TM}}$ II Enzyme Mix from Thermo Scientific.

\subsubsection{Site-Directed Mutagenesis}

Mutations were introduced by the QuikChange Lightning Multi Site-Directed Mutagenesis Kit according to the manufacturer's instructions. 


\subsubsection{Transformation}

\subsubsection{Heat shock transformation}

$50 \mu \mathrm{l}$ chemically competent E. coli (XL1-blue or BL21 (DE3)) cells were thawed on ice. Incubated on ice with $1.42 \mathrm{mM} \beta$-Mercaptoethanol for $10 \mathrm{~min}$ and 5-10 ng DNA for $30 \mathrm{~min}$. Cells were heat shocked at $42{ }^{\circ} \mathrm{C}$ for $45 \mathrm{sec}$, cooled for $1 \mathrm{~min}$ on ice, then $800 \mu 1$ prewarmed SOC medium was added. Bacteria recovered for $60 \mathrm{~min}$ at $37^{\circ} \mathrm{C}$ while shacking, before they were plated on LB agar plates containing appropriate antibiotics.

\subsubsection{Cotransformation}

$20 \mu 1$ chemically competent E. coli Rosetta 2 (DE3) cells were thawed on ice. Incubated on ice with $5 \mathrm{ng}$ of each plasmid for $5 \mathrm{~min}$. Cells were heat shocked at $42{ }^{\circ} \mathrm{C}$ for $30 \mathrm{sec}$, cooled for 2 min on ice, then $100 \mu 1$ prewarmed SOC medium was added. Bacteria recovered for $60 \mathrm{~min}$ at $37{ }^{\circ} \mathrm{C}$ while shacking, before they were plated on LB agar plates containing appropriate antibiotics.

\subsubsection{Transformation for GIBSON Assembly and mutagenesis}

Transformation for GIBSON Assembly and mutagenesis was performed with competent cells provided with the kits according to the manufacturer's instructions. $45 \mu$ l ultracompetent E. coli (XL10-gold) cells provided with the QuikChange Lightning Multi Site-Directed Mutagenesis Kit or $45 \mu \mathrm{l}$ NEB ${ }^{\circledR}$ 5-alpha Competent E. coli provided with the Gibson Assembly® Cloning Kit were thawed on ice. Incubated on ice with $2 \mu 1$ of provided $\beta$ Mercaptoethanol for $2 \mathrm{~min}$ and $2 \mu \mathrm{l}$ of reaction for $30 \mathrm{~min}$. Cells were heat shocked at $42{ }^{\circ} \mathrm{C}$ for $30 \mathrm{sec}$, cooled for $2 \mathrm{~min}$ on ice, then $500 \mu \mathrm{l}$ prewarmed SOC medium was added. Bacteria recovered for $60 \mathrm{~min}$ at $37{ }^{\circ} \mathrm{C}$ while shaking, before they were plated on LB agar plates containing appropriate antibiotics.

\subsubsection{Sequencing of plasmids}

To verify the correct sequence plasmids were sent for single tube sequencing to Eurofins Genomics or SEQLAB Sequence Laboratories Göttingen GmbH using appropriate primers.

\subsubsection{Gel electrophoresis}

\section{Schägger gel electrophoresis}

Almost all proteins used in this study are smaller than $40 \mathrm{kDa}$. Therefore, Schägger gel electrophoresis which has an optimal resolution for proteins smaller than $30 \mathrm{kDa}$ was used instead of normal SDS gel electrophoresis [120]. Schägger gels are two-phase polyacrylamide gels made of a stacking (25\% stacking gel buffer, $61.4 \%$ water, $12.5 \%$ acrylamide, 
$1 \%$ APS, TEMED) and a resolving gel (25\% resolving gel buffer, $34 \%$ water, $40 \%$ acrylamide, $1 \%$ APS, TEMED). Before electrophoresis, protein samples were mixed with Sample buffer for Schägger gel electrophoresis and incubated for $5 \mathrm{~min}$ at $95{ }^{\circ} \mathrm{C}$. Anode and cathode buffer for Schägger gel electrophoresis were used. Electrophoretic separation was done at $70 \mathrm{~V}$ until the sample buffer dye reached the stacking gel. Voltage was switched to $140 \mathrm{~V}$ until the sample buffer dye reached to very bottom of the gel.

Gels were stained with Coomassie staining and destaining solutions heated in microwave for 1 min according to table 2.2.4. and stored in $\mathrm{H}_{2} \mathrm{O}$.

Table 2.2.4.: Coomassie staining protocol

\begin{tabular}{|c|l|c|}
\hline Step & \multicolumn{1}{|c|}{ Solution } & Incubation time \\
\hline 1 & Coomassie staining solution A & $20 \mathrm{~min}$ \\
2 & Coomassie staining solution B & $1 \mathrm{~min}$ \\
3 & Coomassie staining solution C & $1 \mathrm{~min}$ \\
4 & Coomassie destaining solution D & $30 \mathrm{~min}$ \\
\hline
\end{tabular}

\section{SDS PAGE}

To separate lager proteins precasted NuPAGETM 4-12\% Bis-Tris Protein Gels with NuPAGE MOPS SDS Running Buffer (Thermo Scientific) were used according to the manufacturer's instructions.

\section{Native gel electrophoresis}

To analyze cross-linking results native gel electrophoresis was done using precasted RunBlue Native Mini Protein Gels $10 \%$ (Expedeon) according to the manufacturer's instructions.

\subsubsection{Western blot}

For Western Blotting analysis resolved gels were blotted to nitrocellulose membranes by semidry transfer. Gels, membranes and Whatman filter papers were moistened in western blot transfer buffer before assembly. Blotting was performed for $60 \mathrm{~min}$ at a constant voltage of $25 \mathrm{~V}$. After blotting membranes were washed with PBS for 10 min. Next membranes were blocked with $3 \%$ BSA or $1 \%$ skimmed milk in PBS for 1 hour at RT. Membranes were washed with two times PBST and once PBS for 10 min each and incubated with primary antibody in $3 \%$ BSA or $1 \%$ skimmed milk in PBS for 1 hour at RT. Washing was repeated and membranes were incubated with secondary antibody in $3 \%$ BSA or $1 \%$ skimmed milk in PBS for 1 hour at RT. After another round of washing Western Lightening Plus-ECL solution 
was added and the chemiluminiscence signal was detected with Imageready LAS-1000 CCD camera (Fujifilm).

\subsubsection{Protein expression and purification}

Specific expression purification protocols were developed for each complex individually.

\subsubsection{Selenomethionine labeling of proteins}

Proteins were labeled with selenomethionine by expression in M9 minimal medium. M9 medium was mixed by recipe in table 2.1.3., supplemented with appropriate antibiotics and prewarmed at $37{ }^{\circ} \mathrm{C}$. For inoculation $15 \mathrm{ml}$ of an LB overnight culture was spun down at $3000 \mathrm{rpm}$ at $4{ }^{\circ} \mathrm{C}$ to remove LB medium and resuspended in minimal medium. The culture was grown at $37{ }^{\circ} \mathrm{C}$ until $\mathrm{OD}_{600}$ of 0.3 . At this time the temperature was switched to $25^{\circ} \mathrm{C}$ and the amino acid mixture and solid selenomethionine was added. $15 \mathrm{~min}$ later expression was induced with $1 \mathrm{mM}$ IPTG and cultures were grown over night at $25{ }^{\circ} \mathrm{C}$. Cells were harvested by centrifugation with a JS-4.2 rotor in a Beckman J6-MI centrifuge at $4000 \mathrm{rpm}$, $4{ }^{\circ} \mathrm{C}$ for $20 \mathrm{~min}$. Pellets were resuspended in appropriate buffer and stored frozen at $-20{ }^{\circ} \mathrm{C}$. Selenomethionine labeled proteins were purified as described for native proteins but all buffers were supplemented with $1 \mathrm{mM} \beta$-mercaptoethanol

\subsubsection{Rab33B-Atg16L1 E. coli expression and purification}

pETDuet-1 mRab33B or mRab33B-mAtg16L1 constructs or pET28a mAtg16L1 constructs were transformed in E. coli BL21 competent cells. Single cell colonies were picked and transferred to LB medium with appropriate antibiotics and grown over night at $37{ }^{\circ} \mathrm{C}$. For pETDuet-1 Rab33B or Rab33B-mAtg16L1 constructs auto induction medium (see table 2.1.3.) with ampicillin was induced with $1: 100$ overnight culture and grown at $37{ }^{\circ} \mathrm{C}$ until $\mathrm{OD}_{600}$ 0.3. Then temperature was switched to $25{ }^{\circ} \mathrm{C}$ and cells let grown over night. For pET28a mAtg16L1 constructs TB medium was induced with 1:100 $\mathrm{ml}$ overnight culture and grown at $37{ }^{\circ} \mathrm{C}$. At $\mathrm{OD}_{600} 0.3$ cultures were induced with $1 \mathrm{mM}$ IPTG for $4 \mathrm{~h}$ of expression. All cells were harvested by centrifugation with a JS-4.2 rotor in a Beckman J6-MI centrifuge at 4,000 rpm, $4{ }^{\circ} \mathrm{C}$ for $20 \mathrm{~min}$. Pellets were resuspended in $50 \mathrm{mM}$ HEPES pH 7.5, $250 \mathrm{mM}$ $\mathrm{NaCl}, 30 \mathrm{mM}$ imidazole, $5 \mathrm{mM} \mathrm{MgCl} 2,1 \mathrm{mM}$ TCEP and stored frozen at $-20{ }^{\circ} \mathrm{C}$.

For large scale purification cell pellets were thawed and supplemented with a PI tablet, DNaseI, lysozyme and $1 \mathrm{mM} \mathrm{MgCl}_{2}$. Cell suspension was stirred for $15 \mathrm{~min}$ at room temperature. Cell suspension was homogenized with a homogenizer and lysed with three repetitions in a microfluidizer $\mathrm{M}-110 \mathrm{~L}$ (Microfluidics Corporation) according to manufacturer's instructions. Cell debris was pelleted at $16000 \mathrm{rpm}$ and $4{ }^{\circ} \mathrm{C}$ by $45 \mathrm{~min}$ 
centrifugation with a SS-34 rotor in a Du Pont Sorvall centrifuge. The supernatant was filtered with $0.45 \mathrm{~mm}$ filter and filled in a $150 \mathrm{ml}$ Superloop (GE Healthcare) and applied to a $5 \mathrm{ml}$ HisTrap column (GE Healthcare) connected to the Äkta Prime FPLC system at $4{ }^{\circ} \mathrm{C}$ using $50 \mathrm{mM}$ HEPES pH 7.5, $250 \mathrm{mM} \mathrm{NaCl}, 30 \mathrm{mM}$ imidazole, $5 \mathrm{mM} \mathrm{MgCl}_{2}, 1 \mathrm{mM}$ TCEP. Supernatant was loaded onto the column at a flow rate of $1 \mathrm{~mL} / \mathrm{min}$, then the column was washed with $13 \mathrm{CV}$ buffer at $1.5 \mathrm{ml} / \mathrm{min}$. His tagged protein complexes were eluted with a gradient at $1 \mathrm{ml} / \mathrm{min}$ with over $12 \mathrm{CV}$ to $100 \% 50 \mathrm{mM}$ HEPES $\mathrm{pH} 7.5,250 \mathrm{mM} \mathrm{NaCl}$, $400 \mathrm{mM}$ imidazole, $5 \mathrm{mM} \mathrm{MgCl} 2,1 \mathrm{mM}$ TCEP. Elution fractions containing the protein were collected and concentrated to $5 \mathrm{ml}$ in a $10000 \mathrm{MWCO}$ concentrator. Concentrated elution fractions were filled in a $5 \mathrm{ml}$ loop (GE Healthcare) and applied to a Superdex 200 16/60 HiLoad column connected to the Äkta Prime FPLC system at $4{ }^{\circ} \mathrm{C}$. Size exclusion chromatography was run at a flow rate of $1 \mathrm{ml} / \mathrm{min}$ with $30 \mathrm{mM}$ HEPES pH 7.5, $150 \mathrm{mM}$ $\mathrm{NaCl}, 2 \mathrm{mM} \mathrm{MgCl}_{2}$, and $1 \mathrm{mM}$ TCEP. Fractions containing the desired proteins were collected and concentrated in a $3000 \mathrm{MWCO}$ concentrator to $30-45 \mathrm{mg} / \mathrm{ml}$. Purified proteins were aliquoted, flash cooled with liquid nitrogen and stored at $-80{ }^{\circ} \mathrm{C}$ until usage.

\subsubsection{Rab33B-Atg16L1 HEK 293T cell expression and transfection}

The mammalian cell lines HEK 293T cells and COS-7 cells were cultured in DMEM (Dulbecco's Modified Eagle Medium, Lonza) supplemented with $10 \%$ Fetal bovine serum (FBS), $2 \mathrm{mM} \mathrm{L-glutamine} \mathrm{and} 100$ units/ml penicillin/streptomycin. ARPE-19 cells were grown in DMEM/F-12 (Dulbecco's Modified Eagle Medium/Nutrient Mixture F-12, Sigma Aldrich) with the same supplements. Cells were maintained in 10-cm petri dishes for 2-3 days at $37{ }^{\circ} \mathrm{C}$ and $90 \%$ humidity in $5 \% \mathrm{CO}_{2}$. When cells reached the confluence of $80 \%-90 \%$ they were passaged and diluted. Mammalian cells were transiently transfected using LipofectaminTM 2000 (Invitrogen) according to the manufacturer's instructions. $50 \mu \mathrm{l}$ of Lipofectamine was diluted in $1.5 \mathrm{ml}$ DMEM without supplement for $5 \mathrm{~min}$ at RT and then combined with $12 \mu \mathrm{g}$ of DNA diluted in $1.5 \mathrm{ml}$ of DMEM without supplement. This transfection mix was then incubated for an additional $20 \mathrm{~min}$ at room temperature. Subsequently the mixture was added onto the cells and then incubated for $24 \mathrm{~h}$ in the cell incubator. After $24 \mathrm{~h}$, transfected cells were lysed using $600 \mu \mathrm{l}$ of lysis buffer containing $50 \mathrm{mM}$ HEPES pH 7.4, $150 \mathrm{mM} \mathrm{NaCl}, 1 \mathrm{mM} \mathrm{MgCl} 2,1 \%$ Triton-X-100) for 15 min at $4{ }^{\circ} \mathrm{C}$. The lysate was collected using scraper and transferred into $2 \mathrm{ml}$ Eppendorf tube and centrifuged at $10.000 \mathrm{~g}$ for $10 \mathrm{~min}$. The supernatant was used for co-immunoprecipitation. 
To examine the expression levels transfected cells were lysed and the supernatant was submitted to SDS PAGE electrophoresis and western blotting. Membranes were probed with rabbit anti-GFP (1:10 000) or mouse anti-V5 (1:1 000) primary antibody and goat anti rabbit or goat anti mouse IR dye 800 (1:15 000) secondary antibody for Odyssey fluorescence detection (LI-COR Odyssey CLx Infrared Imaging System). Rab33B-Atg16L1 HEK 293T cell expression and transfection were done by Dr. Beyenech Binnotti (Department for Neurobiology).

\subsubsection{KlAtg21-AgAtg16 expression and purification}

pET28a KlAtg21 or pET28a AgAtg16 constructs were transformed in E. coli BL21 competent cells. Single cell colonies were picked and transferred to LB medium with appropriate antibiotics and grown over night at $37{ }^{\circ} \mathrm{C}$. Auto induction medium (see table 2.1.3.) with appropriate medium was induced with 1:100 overnight culture and grown at $37{ }^{\circ} \mathrm{C}$ until $\mathrm{OD}_{600} 0.3$. Then temperature was switched to $25{ }^{\circ} \mathrm{C}$ and cells let grown over night. Cells were harvested by centrifugation with a JS-4.2 rotor in a Beckman J6-MI centrifuge at $4000 \mathrm{rpm}$, $4{ }^{\circ} \mathrm{C}$ for $20 \mathrm{~min}$. Pellets were resuspended in $50 \mathrm{mM}$ HEPES pH 7.5, $250 \mathrm{mM} \mathrm{NaCl}, 30 \mathrm{mM}$ imidazole, $1 \mathrm{mM}$ TCEP and stored frozen at $-20{ }^{\circ} \mathrm{C}$.

For purification cell pellets were thawed and supplemented with a PI tablet, DNaseI, lysozyme and $1 \mathrm{mM} \mathrm{MgCl}$. For complex purification cell pellets of KlAtg21 and AgAtg16 were mixed in a 2:1 ratio. Cell suspension was stirred for $15 \mathrm{~min}$ at room temperature. Cell suspension was homogenized with a homogenizer and lysed with three repetitions in a microfluidizer M-110L (Microfluidics Corporation) according to manufacturer's instructions. Cell debris was pelleted at $16000 \mathrm{rpm}$ and $4{ }^{\circ} \mathrm{C}$ by 45 min centrifugation with a SS-34 rotor in a Du Pont Sorvall centrifuge. The supernatant was filtered with $0.45 \mathrm{~mm}$ filter and filled in a $150 \mathrm{ml}$ Superloop (GE Healthcare) and applied to a $5 \mathrm{ml}$ HisTrap column (GE Healthcare) connected to the Äkta Prime FPLC system at $4{ }^{\circ} \mathrm{C}$ using $50 \mathrm{mM}$ HEPES pH 7.5, $250 \mathrm{mM}$ $\mathrm{NaCl}, 30 \mathrm{mM}$ imidazole, $1 \mathrm{mM}$ TCEP. Supernatant was loaded onto the column at a flow rate of $1 \mathrm{ml} / \mathrm{min}$, then the column was washed with $12 \mathrm{CV}$ buffer at $1.5 \mathrm{ml} / \mathrm{min}$. His tagged protein complexes were eluted with a gradient at $1 \mathrm{ml} / \mathrm{min}$ with $10 \mathrm{CV}$ of using $50 \mathrm{mM}$ HEPES pH 7.5, $250 \mathrm{mM} \mathrm{NaCl}, 400 \mathrm{mM}$ imidazole, $1 \mathrm{mM}$ TCEP. Elution fractions containing the desired protein were collected and concentrated to $5 \mathrm{ml}$ in a $10000 \mathrm{MWCO}$ concentrator. Concentrated elution fractions were filled in a $5 \mathrm{ml}$ loop (GE Healthcare) and applied to a Superdex 200 16/60 HiLoad column connected to the Äkta Prime FPLC system at $4{ }^{\circ} \mathrm{C}$. Size exclusion chromatography was run at a flow rate of $1 \mathrm{ml} / \mathrm{min}$ with $30 \mathrm{mM}$ HEPES $\mathrm{pH} 7.5$, $150 \mathrm{mM} \mathrm{NaCl}, 1 \mathrm{mM}$ TCEP. Fractions containing the desired proteins were collected and 
concentrated in a $3000 \mathrm{MWCO}$ concentrator to $10-30 \mathrm{mg} / \mathrm{ml}$. Purified proteins were aliquoted, flash cooled with liquid nitrogen and stored at $-80^{\circ} \mathrm{C}$ until usage.

\subsubsection{SCOC-FEZ1 co-expression and co-purification}

pET28a SCOC and pET22b FEZ1 were co-transformed in E. coli Rosetta ${ }^{\mathrm{TM}}$ 2(DE3)pLysS competent cells. Single cell colonies were picked and transferred to LB medium with appropriate antibiotics and grown over night at $37^{\circ} \mathrm{C}$. LB expression cultures were induced with 1:100 ml overnight culture and grown at $37^{\circ} \mathrm{C}$. At $\mathrm{OD}_{600} 0.3$ cultures were induced with $1 \mathrm{mM}$ IPTG. After 3 to $4 \mathrm{~h}$ of expression cells were harvested by centrifugation with a JS-4.2 rotor in a Beckman J6-MI centrifuge at $4000 \mathrm{rpm}, 4{ }^{\circ} \mathrm{C}$ for $20 \mathrm{~min}$. Pellets were resuspended in $50 \mathrm{mM}$ HEPES $\mathrm{pH} 7.5,250 \mathrm{mM} \mathrm{NaCl}$ and stored frozen at $-20{ }^{\circ} \mathrm{C}$.

For co-purification of Strep-tagged SCOC with His-tagged FEZ1, cell pellets were thawed and supplemented with a PI tablet, DNaseI, lysozyme and $1 \mathrm{mM} \mathrm{MgCl}$. Cell suspension was stirred for $15 \mathrm{~min}$ at room temperature. Cell suspension was homogenized with a homogenizer and lysed with three repetitions in a microfluidizer M-110L (Microfluidics Corporation) according to the manufacturer's instructions. Cell debris was pelleted at $16000 \mathrm{rpm}$ and $4{ }^{\circ} \mathrm{C}$ by $45 \mathrm{~min}$ centrifugation with a SS-34 rotor in a Du Pont Sorvall centrifuge. The supernatant was filtered with $0.45 \mathrm{~mm}$ filter and imidazole concentration adjusted to $30 \mathrm{mM}$. The supernatant was incubated with $0.5 \mathrm{ml} \mathrm{Ni-NTA}$ Sepharose per $10 \mathrm{ml}$ supernatant for $1 \mathrm{~h}$ at $4{ }^{\circ} \mathrm{C}$ under rotation. Ni-NTA Sepharose was washed three times with 5 times sepharose volume of $50 \mathrm{mM}$ HEPES pH 7.5, $250 \mathrm{mM} \mathrm{NaCl}, 30 \mathrm{mM}$ imidazole. His tagged protein was eluted with 3 times sepharose volume of $50 \mathrm{mM}$ HEPES pH 7.5, $250 \mathrm{mM}$ $\mathrm{NaCl}, 400 \mathrm{mM}$ imidazole. Elution was immediately filled in a $50 \mathrm{ml}$ Superloop (GE Healthcare) and applied to a $5 \mathrm{ml}$ StrepTrap column (GE Healthcare) connected to the Äkta Prime FPLC system at $4{ }^{\circ} \mathrm{C}$ using $50 \mathrm{mM}$ HEPES pH 7.5, $250 \mathrm{mM} \mathrm{NaCl}$. Supernatant was loaded onto the column at a flow rate of $1 \mathrm{ml} / \mathrm{min}$, then the column was washed with $7 \mathrm{CV}$ buffer at $1.5 \mathrm{ml} / \mathrm{min}$. Strep tagged protein complexes were eluted at $1 \mathrm{ml} / \mathrm{min}$ with $6 \mathrm{CV}$ of buffer supplemented with $2.5 \mathrm{mM}$ desthiobiotin. Elution fractions containing the protein were collected and concentrated to $5 \mathrm{ml}$ in a $3000 \mathrm{MWCO}$ concentrator. Concentrated elution fractions were filled in a $5 \mathrm{ml}$ loop (GE Healthcare) and applied to a Superdex 75 16/60 HiLoad column connected to the Äkta Prime FPLC system at $4{ }^{\circ} \mathrm{C}$. Size exclusion chromatography was run at a flow rate of $1 \mathrm{ml} / \mathrm{min}$ with $30 \mathrm{mM}$ HEPES $\mathrm{pH} 7.5,150 \mathrm{mM}$ $\mathrm{NaCl}$. Fractions containing the protein complex were collected and concentrated in a 3000 MWCO concentrator to $10-15 \mathrm{mg} / \mathrm{ml}$. Purified proteins were aliquoted, flash cooled with liquid nitrogen and stored at $-80{ }^{\circ} \mathrm{C}$ until usage. 


\subsubsection{His-tag cleavage by thrombin}

Cloning of SCOC in the pET28a and FEZ1 in the pET22b vector included a thrombin cleavage site between protein sequence and the tag [56]. After gel filtration both tags were cleaved for one purification batch. Pooled fractions were dialyzed at $4{ }^{\circ} \mathrm{C}$ overnight in a Spectra/Pore dialysis membrane (Spectrumlabs.com) with gel filtration buffer containing $10 \mathrm{U}$ of thrombin (MP Biomedicals) per mg of protein. After dialysis, thrombin was inhibited by adding $4 \mathrm{mM}$ Pefabloc SC (Roche), a serine protease inhibitor.

\subsubsection{Measurement of protein concentration}

For SCOC-FEZ1 complex and Rab33B-mAtg16L1 complex protein concentrations were usually determined using the Bradford assay [17]. Protein solution was diluted to appropriate concentrations with $\mathrm{H}_{2} \mathrm{O}$ to a final volume of $200 \mu \mathrm{l} .800 \mu \mathrm{l}$ Bradford working solution (500 ml contained: $425 \mathrm{ml} \mathrm{H} 2 \mathrm{O}, 15 \mathrm{ml} 95 \%$ ethanol, $30 \mathrm{ml} 88 \%$ phosphoric acid) was added and the absorbance was measured at $595 \mathrm{~nm}$ on a Genesys 6 spectrophotometer (Thermo Spectronic). The concentration was determined from a BSA standard curve using 1 to $10 \mu \mathrm{g}$ BSA.

In the case of KlAtg21 and AgAtg16 the protein concentration was spectroscopically measured using an ND-1000 spectrophotometer (NanoDrop Technologies Inc.) which uses a path length of $0.2 \mathrm{~mm}$ and measured the absorbance of aromatic amino acid residues at $280 \mathrm{~nm}$ wavelength using the molecular weight and extinction coefficient of the protein

\subsubsection{Analytic gel filtration}

To analyze complex formation size exclusion chromatography with a Superdex ${ }^{\text {TM }} 200$ 10/300 GL column (analytic gel filtration) connected to a Äkta Purifier FPLC system at RT was performed. The column was equilibrated with gel filtration buffer used for protein purification. Proteins were incubated alone or together with the potential complex partner prior analysis for $30 \mathrm{~min}$ at $4{ }^{\circ} \mathrm{C}$ in a total volume of $400 \mu \mathrm{l}$. Proteins were injected in a $500 \mu 1$ loop and loaded on the column at a flowrate of $0.5 \mathrm{ml} / \mathrm{min}$. The column was run with 1.25 CV gel filtration buffer at a flowrate of $0.5 \mathrm{ml} / \mathrm{min}$ and $0.5 \mathrm{ml}$ fractions were collected. Chromatograms of single proteins and proteins mixtures were compared and fractions were analyzed by Schägger gel electrophoresis.

\subsubsection{Limited proteolysis}

Limited proteolysis was performed using the Proti-Ace I + II (Hampton Research) Kits following the manufacturer's instructions. Proteins were mixed with 1:1000 proteases (Proteinase-K, Clostripain, Pepsin, Thermolysin, Bromelain, Actinase E, $\alpha$-Chymotrypsin, 
Trypsin, Elastase, Papain, Substilisin or Endoproteinase Glu-C). The degradation process was followed by taking samples at different time points. Reactions were stopped by adding Schägger gel sample buffer and heating to $95{ }^{\circ} \mathrm{C}$ for $5 \mathrm{~min}$. Limited proteolysis was analyzed by Schägger gel electrophoresis.

\subsubsection{Circular dichroism spectroscopy}

Circular dichroism spectra were recorded with a Chirascan Circular Dichroism spectrometer (AppliedPhotophysics) using Hellma quartz cuvettes with a path length of $0.1 \mathrm{~cm}$. Samples were buffer exchanged to CD buffer before measurements. Far UV CD spectra of $1 \mathrm{mg} / \mathrm{ml}$ protein were recorded from 180 to $260 \mathrm{~nm}$ with a step size of $0.5 \mathrm{~nm}$, a bandwidth of $0.5 \mathrm{~nm}$ and $3 \mathrm{sec}$ time per point at $20^{\circ} \mathrm{C}$. Thermal melts were determined from 20 to $90{ }^{\circ} \mathrm{C}$ in $0.2{ }^{\circ} \mathrm{C}$ steps with a heating rate of $0.5{ }^{\circ} \mathrm{C} / \mathrm{min}$ at $222 \mathrm{~nm}$ for $\alpha$-helix and $215 \mathrm{~nm}$ for $\beta$-sheet containing proteins. Data were analyzed with the manufacturer's ProView Software.

\subsubsection{ProteoPlex assay}

ProteoPlex [23] is a stabilizing assay method for macromolecular complexes based on the thermal shift assay (ThermoFluor) [34]. To find optimal stabilizing conditions proteins are heated in 96 different buffer conditions by a high-throughput system. Unfolding events are monitored with SYPRO Orange dye. ProteoPlex measurements were conducted by Dr. David Haselbach in the lab of Prof. Holger Stark (Department for Structural Dynamics, MPI for Biophysical Chemistry, Göttingen).

\subsubsection{SEC-MALLS}

The stoichiometry of complexes can be analyzed by size exclusion chromatography coupled to a Multi Angle Laser Light Scatterer (SEC-MALLS). For SEC-MALLS measurements purified proteins were loaded on a Superdex 10/300 GL column connected to an Äkta Purifier FPLC system. The instrument was equilibrated with gel filtration buffer used during purification. The size exclusion column is connected to a miniDAWN Treos multi-angle light scattering detector and an Optilab T-rEX refractive index concentration detector (Wyatt Technologies). Results were evaluated with the program ASTRA® 6 by Wyatt Technologies. SEC-MALLS measurements were conducted by Johannes Arens and Dr. Achim Dickmanns in the group of Prof. Ralf Ficner (Department for Molecular Structural Biology, University of Göttingen). 


\subsubsection{Isothermal titration calorimetry}

Isothermal titration calorimetry (ITC) measurements were accompanied using the MicroCal iTC200 (GE Healthcare Life Sciences) set up. Prior to ITC measurements proteins were dialyzed overnight in the same buffer, spun down at $10000 \mathrm{rpm}$ for $10 \mathrm{~min}$ at $4{ }^{\circ} \mathrm{C}$ and concentrations were adjusted. 25 total injections of $2 \mu \mathrm{l}$ were performed with $120 \mathrm{~s}$ delay. Temperature was set to $20^{\circ} \mathrm{C}$, stirring speed to $300 \mathrm{rpm}$ and reference power to $7 \mu \mathrm{cal} / \mathrm{s}$. ITC measurements were conducted by Dr. Angel Perez Lara (Department for Neurobiology, MPI for Biophysical Chemistry, Göttingen) with an instrument in the group of Prof. Marina Rodnina (Department for Physical Biochemistry, MPI for Biophysical Chemistry, Göttingen).

\subsubsection{Cross-linking and analysis by mass spectrometry}

Protein complexes were cross-linked with bis(sulfosuccinimidyl) suberate (BS3). $200 \mu 1$ purified protein complexes $(5 \mathrm{mg} / \mathrm{ml})$ were incubated with $0,0.25,0.5,1,2$ and $4 \mathrm{mM} \mathrm{BS3}$ for $30 \mathrm{~min}$ at RT under constant rotation. The reaction was stopped by the addition of $1 \mu \mathrm{L}$ of $1 \mathrm{M}$ Tris-HCl, $\mathrm{pH}$ 8. Samples were run on a NativePAGETM Bis-Tris Gel (Thermo Scientific). Gels were sent to the Bioanalytical Mass Spectrometry Facility. Bands of interest were cut from the gels and redissolved in $50 \mathrm{mM} \mathrm{NH}_{4} \mathrm{HCO}_{3}, 4 \mathrm{M}$ urea, $\mathrm{pH}$ 8.0. Proteins were digested with trypsin of 1/20 complex amount (w/w) overnight. Subsequently, the peptide fragments were desalted and fractionated by a Superdex Peptide 10/300 GL size exclusion column (GE Healthcare) using a solvent system containing 30\% acetonitrile $(\mathrm{ACN}), 0.1 \%$ trifluoroacetic acid (TFA) (v/v). The fragments were collected in $50 \mu \mathrm{L}$ fractions, lyophilized, reconstituted in $20-30 \mu \mathrm{L}$ of $5 \% \mathrm{ACN}, 0.1 \%$ formic acid (FA), and subjected to Liquid chromatographytandem mass spectrometry (LC-MS/MS) analysis.

Six microliters of the cross-linking sample was injected onto a nano-liquid chromatography system (UltiMateTM 3000 RSLCnano system) including a $3 \mathrm{~cm} \times 150 \mu \mathrm{m}$ inner diameter C18 trapping column in-line with a $30 \mathrm{~cm} \times 75 \mu \mathrm{m}$ inner diameter $\mathrm{C} 18$ analytical column (both in-house packed with 1.9- $\mu \mathrm{m} \mathrm{C18} \mathrm{material,} \mathrm{Dr.} \mathrm{Maisch} \mathrm{GmbH}$ ). Peptides were loaded on the trapping column, desalted for $3 \mathrm{~min}$ at a flow rate of $10 \mu \mathrm{L} / \mathrm{min}$ in $95 \%$ of mobile phase $\mathrm{A}\left(0.1 \%\right.$ FA in $\left.\mathrm{H}_{2} \mathrm{O},(\mathrm{v} / \mathrm{v})\right)$ and $5 \%$ of mobile phase $\mathrm{B}(80 \% \mathrm{ACN}$ and $0.05 \%$ FA in $\mathrm{H}_{2} \mathrm{O}$, (v/v)). After desalting, peptides were eluted and separated on the analytical column using a 43-min linear gradient of 15-46 \% mobile phase B at a flow rate of $300 \mathrm{nl} / \mathrm{min}$. Separated peptides were analyzed on-line on an Orbitrap Fusion mass spectrometer (Thermo Scientific). A top-20 method was employed were the 20 most intense precursor ions with charge states 3-8 in the survey scan $(380-1580 \mathrm{~m} / \mathrm{z}$ scan range) were isolated in the quadrupole mass filter (isolation window $1.6 \mathrm{~m} / \mathrm{z}$ ) and fragmented in the higher energy 
collisional dissociation (HCD) cell with normalized energy 30. A dynamic exclusion of $20 \mathrm{~s}$ was used. Both the survey scan (MS1) and the product ion scan (MS2) were performed in the Orbitrap at 120,000 and 30,000 resolution, respectively. Spray voltage was set at $2.3 \mathrm{kV}$ and $60 \%$ of S-lens RF level was used. Automatic gain control (AGC) targets were set at $5 \times 105$ (MS1) and 5×104 (MS2). The raw data were converted to mgf files by Proteome Discoverer 2.0.0.802 software (Thermo Scientific). The mgf files were searched against a FASTA database containing the sequences protein complex components by pLink 1.22 software using a target-decoy strategy. Database search parameters included mass accuracies of MS1<10 ppm and MS2<20 ppm, carbamidomethylation on cysteine as a fixed modification, oxidation on methionine as variable modification. Number of residues of each peptide on a cross-link pair was set between 4 and 40. A maximal of two trypsin missed-cleavage sites was allowed. The results were obtained with $1 \%$ false discovery rate. Visualization of cross-links was made with xiNET [26]. To overcome false positive cross-links the threshold for the maximal score was set to 5.1 .

This work was done in collaboration with Dr. Momchil Ninov (Department of Neurobiology, MPI for Biophysical Chemistry, Göttingen) and Dr. Chung-Tien Lee with technical support of Monika Raabe in the group of Prof. Henning Urlaub (Bioanalytical Mass Spectrometry, MPI for Biophysical Chemistry, Göttingen).

\subsubsection{Ni-Sepharose pulldown}

To analyze complex formation of Rab33B-Atg16L1 by Ni-Sepharose pulldown the complex was expressed in E. coli cells and solubilized as described before (2.2.5.2.). In the meantime Ni-NTA Sepharose beads (GE Healthcare) were washed with $\mathrm{H}_{2} \mathrm{O}$ and buffer A $(50 \mathrm{mM}$ HEPES pH 7.5, $250 \mathrm{mM} \mathrm{NaCl}, 30 \mathrm{mM}$ imidazole, $5 \mathrm{mM} \mathrm{MgCl}_{2}, 1 \mathrm{mM}$ TCEP). $10 \mathrm{ml}$ supernatant from $E$. coli expression was incubated with $1 \mathrm{ml} \mathrm{Ni-Sepharose} \mathrm{beads} \mathrm{for} \mathrm{one} \mathrm{hour}$ under constant rotation at $4{ }^{\circ} \mathrm{C}$. Beads were centrifuged at $3000 \mathrm{rpm}$ for $5 \mathrm{~min}$ at $4{ }^{\circ} \mathrm{C}$ and the flow trough was removed. Beads were washed three times with $5 \mathrm{ml}$ buffer A. Finally proteins were eluted two times with $1.5 \mathrm{ml}$ buffer B (50 mM HEPES pH 7.5, $250 \mathrm{mM} \mathrm{NaCl}$, $400 \mathrm{mM}$ imidazole, $5 \mathrm{mM} \mathrm{MgCl}_{2}, 1 \mathrm{mM}$ TCEP). Samples were taken from cell pellet (P), supernatant (S), flow through (ft), wash steps 1-3 (W1-3) and elution (E1-2) mixed with 3x Schägger gel buffer and boiled for $5 \mathrm{~min}$ at $95{ }^{\circ} \mathrm{C}$. Purification steps were analyzed by Schägger gel electrophoresis and stained with Coomassie. Elution fractions were pooled and analyzed by Schägger gel electrophoresis followed by western blot. Membranes were blocked in $3 \%$ BSA and probed with Penta His HRP conjugate antibody (1:1500) to detect His-tagged Rab33B or blocked in $5 \%$ skimmed milk and probed with rabbit anti-Atg16L primary 
antibody (1:2000) and goat anti-rabbit IgG (HRP labeled) (1:1000) secondary antibody to detect Atg16L1.

\subsubsection{Mammalian cell line culture}

The mammalian cell lines HEK 293T cells and COS-7 cells were cultured in DMEM (Dulbecco's Modified Eagle Medium, Lonza) supplemented with $10 \%$ Fetal bovine serum (FBS), $2 \mathrm{mM} \mathrm{L-glutamine} \mathrm{and} 100$ units/ml penicillin/streptomycin. ARPE-19 cells were grown in DMEM/F-12 (Dulbecco's Modified Eagle Medium/Nutrient Mixture F-12, Sigma Aldrich) with the same supplements. Cells were maintained in 10-cm petri dishes for 2-3 days at $37{ }^{\circ} \mathrm{C}$ and $90 \%$ humidity in $5 \% \mathrm{CO}_{2}$. When cells reached the confluence of $80 \%-90 \%$ they were passaged and diluted.

\subsubsection{Co-immunoprecipitation}

The precleared supernatant from transiently transfected HEK 293 T cells (2.2.6.3.) was used for co-immunoprecipitation. $30 \mu \mathrm{l}$ of supernatant was used as input fraction. The rest of the sample was incubated with specific antibody (anti V5 or anti GFP) for $2 \mathrm{~h}$ under constant rotation at $4{ }^{\circ} \mathrm{C}$. In the meantime protein A dynabeads (Thermo Scientific) were washed once with lysis buffer and then kept in the lysis buffer under constant rotation. The supernatant was transferred in the prewashed beads and incubated for an additional hour under constant rotation at $4{ }^{\circ} \mathrm{C}$. The beads were washed three times with $1 \mathrm{ml}$ lysis buffer, transferred to a new tube and washed again two times. Finally proteins were eluted from the beads using $4 \mathrm{x}$ NuPAGE LDS Sample Buffer (Thermo Scientific) in presence of $10 \%$ of beta-mercaptoethanol. Samples were boiled for $10 \mathrm{~min}$ at $70{ }^{\circ} \mathrm{C} .5 \mu \mathrm{l}$ of the input and $10 \mu \mathrm{l}$ of the elution (IP) sample were separated by SDS-Page gel electrophoresis. Proteins were transferred on a PDVF membrane by western blotting. Membranes were cut, blocked with $10 \%$ skimmed milk in PBST and probed with specific primary antibodies rabbit GFP (1:10 000), mouse V5 $(1: 1000)$ or rabbit LC3B (1:1000). For GFP-pulldowns a mouse anti rabbit light chain specific HRP labeled secondary antibody (1:2000) was used to detect the GFP antibody. For all other probes and V5 pull downs either goat anti mouse HRP labeled (1:2000) or goat anti rabbit HRP labeled (1:2000) secondary antibody was used. Co-immunoprecipitation was done with Dr. Beyenech Binnotti (Department for Neurobiology).

\subsubsection{Fluorescence microscopy}

Transiently transfected COS-7 or ARPE-19 cells were washed once with PBS to remove serum. The cells were fixed using $4 \%$ paraformaldehyde for $15 \mathrm{~min}$ at RT. The fixative was removed and the cells were washed 3 times 5 min each with PBS. Afterward the cells were 
blocked with $10 \%$ normal goat serum and $0.2 \%$ Triton-X-100 in PBS for $1 \mathrm{~h}$. The coverslips were inverted on top of a drop of $45-50 \mu$ of mouse anti-V5 primary antibody (1:2000) diluted in blocking buffer. The incubation was performed in a dark and humidified chamber for 1 to 2 hours at RT or o/n at $4{ }^{\circ} \mathrm{C}$. The coverslips were washed 3 times for 5 min each with PBS and incubated again following the same procedure with goat anti mouse Cy3 (1:600) secondary antibody for 1 hour at RT. Additional, cells were incubated with DAPI (Thermo Scientific). Finally the cells were washed as previously described and mounted on a microscope slides using a mounting media (Fluoro-Gel, Electron Microscopy Sciences, or VECTASHIELD HardSet Mounting Medium with DAPI, Vector Laboratory). The mounting media was let dry o/n at $4{ }^{\circ} \mathrm{C}$.

Images were acquired using an epifluorescence microscope (Axioverter 200M, ZEISS). ImageJ was used to convert and merge pictures. Fluorescence microscopy was done with Dr. Beyenech Binnotti (Department for Neurobiology).

\subsubsection{MAP1LC3 Lipidation assay}

Transiently transfected HEK 293 T cells (2.2.6.3.) were used for the MAP1LC3 Lipidation assay. 24 hours after transfection cells were treated for 4 hours with $20 \mathrm{mM} \mathrm{NH}_{4} \mathrm{Cl}$ to block the autophagic flux. For control cells were maintained in normal nutrient rich medium. After 4 hours cells were washed once with ice cold PBS, and then lysed for $10 \mathrm{~min}$ with lysis buffer (50 mM HEPES pH 7.4, $150 \mathrm{mM} \mathrm{NaCl}, 1 \mathrm{mM} \mathrm{MgCl} 2,2 \% \mathrm{TX}-100$ and complete protease inhibitor (Roche)). Lysates were collected and precleared by centrifugation at $10000 \mathrm{rpm}$ for $10 \mathrm{~min}$ at $4^{\circ} \mathrm{C}$. Protein concentrations were measured with Pierce BCA reagent (Thermo Scientific). $10 \mu \mathrm{g}$ total protein was separated by $12 \%$ Schägger gel electrophoresis. Proteins were transferred on a PDVF membrane by western blotting. Membranes were cut, blocked with $10 \%$ skimmed milk in PBST and probed with specific primary antibodies rabbit GFP (1:5000), mouse V5 (1:1000), mouse Tubulin (1:2000) or rabbit LC3B (1:1000). Either goat anti mouse IgG IR dye 800 (1:15 000) or goat anti rabbit IgG IR dye 800 (1:15 000) secondary antibody was used for Odyssey fluorescence detection (LI-COR Odyssey CLx Infrared Imaging System). The MAP1LC3 Lipidation assay was done by Dr. Beyenech Binnotti (Department for Neurobiology). 


\subsubsection{Crystallization and structure determination}

\subsubsection{Crystallization screen setup}

\section{6-well sitting drop plates}

Initial crystallization screening experiments were set up in 96-well sitting drop plates (MRC 2 Well Crystallization Plate (Swissci) UVP, Hampton research or MRC 96 well Crystallization Plate $^{\mathrm{TM}}$ UV, Molecular Dimensions) with commercial available crystallization screens. Commercial crystallization screens were purchased from Hampton Research (SaltRx), Qiagen (AmSO4, Anions, Cations, ClassicLite, Classics I+II, ComPAS, JCSG ${ }^{+}$, PACT, PEG I+II, pHclear I+II, Protein Complex) or Emerald Biosystems (Wiz1+2, Wiz3+4 screens).Two drops of different protein concentrations were pipetted for each well. The Cartesian robot was used for setting $60 \mathrm{nl}$ drops of crystallization solution and adding $60 \mathrm{nl}$ protein solution. The Gryphon robot was used for setting $100 \mathrm{nl}$ drops of crystallization solution and adding $1000 \mathrm{nl}$ protein solution. Plates were covered with a transparent sealing tape and stored at 20 or $4{ }^{\circ} \mathrm{C}$ in an automated Formulatrix crystallization imager which imaged the plate on a pre-set schedule at the Crystallization Facility of the MPI for Biophysical Chemistry, Göttingen. Images were checked with the Rockmaker main Application software (Formulatrix).

\section{4-well Linbro hanging drop plates}

When crystallization hits from initial screening were found optimization was done using 24well Linbro hanging drop plates. The original crystallization solution was self-made, $1 \mathrm{ml}$ for every reservoir. For optimization $\mathrm{pH}$, precipitant or salt concentration was differed along row and axis in a grid screen. 1-3 $\mu 1$ protein and 1-3 $\mu 1$ reservoir solution (mother liquor) were pipetted without mixing a siliconized cover slip with up to 4 drops on one slide. The cover slip was flipped upside down onto the greased well and sealed air tight. All chemicals used in the optimization screens were from Sigma, Fluka, or Sigma-Aldrich and were analytical grade with $\geq 99.0 \%$ purity.

\section{Additive and Silver Bullets screen}

Additive screen (HR2-428, Hampton Research) or Silver Bullets (HR2-096) was used for optimization. For 96-well screens $70 \mu 1$ of crystallization solution was filled in every well and mixed with $7 \mu \mathrm{l}$ of additive or Silver Bullets. For 24-well plate screens $1.5 \mu \mathrm{l}$ protein and $1.5 \mu 1$ reservoir solution were pipetted on a cover slip followed by $0.3 \mu$ l additive before placing on the pregreased Linbro plate. 


\section{Streak seeding}

Streak seeding was performed in 24 -well plates to provide nucleation sites. Therefore an existing crystal was crushed with an acupuncture needle. A fresh needle was then streaked first through the crashed crystal and then through the pre-pipetted crystallization drop.

\section{Heavy metal soaking}

Heavy metal soaking was done using the JBS Magic Triangle Phasing Kit (Jena Bioscience). According to the manufacturer's instructions 5-Amino-2,4,6-triiodoiso-phthalic acid (I3C) was incorporated by soaking and co-crystallization. For co-crystallization different concentrations of IC3 $(5,10$ and $15 \mathrm{mM})$ were added to the crystallization solution prior to crystallization. For soaking native crystals were transferred for $10-60$ second to a fresh drop of crystallization solution supplemented with 100, 250 or $500 \mathrm{mM} \mathrm{IC3.}$

\subsubsection{Flash cooling of crystals}

Prior to flash cooling crystals were cryoprotected to prevent ice formation around the crystal. For this $20-30 \%$ of the water content of the respective mother liquor was substituted by cryoprotectants like ethylene glycol, xylitol, sucrose or PEG 400 or mixtures (Cryoprotectant screen). Crystals were fished with mounted round LithopLoops (Molecular Dimensions) and put in fresh mother liquor, then transferred to a mixture of 1:1 mother liquor and cryosolution and finally in pure cryosolution. Crystals were fished out of the cryosolution and quickly flash cooled in liquid nitrogen.

\subsubsection{X-ray diffraction data collection and processing}

$\mathrm{X}$-ray diffraction data were collected at $100 \mathrm{~K}$ on a PILATUS M6 detector at beamline X10SA or on an EIGER 16M detector at beamline X06SA at the Swiss Light Source (Paul Scherrer Institute, Villigen, Switzerland). Diffraction data were processed and scaled with the XDS software package consisting of three programs XDS, XDSSCALE and XDSCONV [59]. $\mathrm{XDS}$ is the main program that performs eight subroutines in order to produce a list of corrected, integrated intensities. XYCORR, INIT and COLSPOT determine and correct the collected spots versus the background. Determined spots are used for indexing by IDXREF to determine orientation, dimensions, and symmetry of the crystal lattice and integrated by INTEGRATE. The final CORRECT step applies correction factors to the measured intensities, refines the unit cell parameters and reports statistics of data completeness and quality. XSCALE is used for scaling and merging of data. XDSCONV converts to specific output formats needed for structure determination programs. Processed data were converted to .mtz file format. 


\subsubsection{Xtriage analysis}

Processed datasets were subjected for analysis by the program Xtriage from the Phenix program suite [1]. Xtriage determines the quality of datasets by analyzing the Matthews coefficient [84] to estimate the number of molecules in the asymmetric unit of a crystal and the intensity of anomalous signal if present. Furthermore it automatically identifies ice rings and twinning.

\subsubsection{Molecular replacement}

For structure determination an electron density map must be calculated by Fourier synthesis. Both structure factors and phases are needed for the calculation. Structure factor amplitudes are directly derived by the measured intensities of the reflection spots. Phases cannot be obtained directly from experimental data. Phases can be directly derived by de novo phasing suitable for high resolution structures or by indirect methods like experimental phasing or molecular replacement. Experimental phasing can be done by single or multiple anomalous diffraction (SAD/ MAD), if an anomalous scatterer is present. Therefore proteins can be labeled with selenomethionine or crystals can be soaked with heavy metals. SAD or MAD data are obtained at wavelengths where anomalous diffraction occurs. Differences between Friedel pairs are measured and used to calculate the position of the anomalous atoms by Patterson methods. To obtain an electron density map by SAD or MAD the anomalous signal must obtain certain intensity.

Structure determination by molecular replacement (MR) requires a search model. Structures with a sequence identity of at least $30 \%$ are potentially suitable for molecular replacement. To minimize the variance of a search model flexible domains or side chains can be truncated. For MR Patterson functions are calculated from both the measured structure factors and the search model. Because the anomalous signal strength was low for AgAtg16 and homologues structures were available for KlAtg21, AgAtg16, mRab33B and mAtg16L1 molecular replacement was used to obtain phases in this study. For AgAtg16 Phaser-MR from the CCP4 program suite and for KlAtg21-AgAtg16 and Rab33B-Atg16L1 Phaser-MR from the Phenix program suite was used [85]. Molecular replacement requires knowledge about the expected number of molecules per asymmetric unit. Multiple search models can be used at the same time or Phaser-MR can search for an additional model in a fixed partial solution. Phaser-MR performs an anisotropy correction to overcome anisotropy, a translational noncrystallographic symmetry (tNCS) correction, a rotation function to determine the orientation and a translational function to calculate the absolute position of the model in the unit cell. 
Fitting of the model is evaluated by a packing analysis that calculates the rotation function Zscore (TFZ) based on the number of clashes between atoms. Phaser-MR performs a simple rigid-body refinement and calculates phases for the electron density map from the positions of the molecules in the asymmetric unit. Finally the log-likelihood gain (LLG) is calculated to evaluate the success of MR. The final TFZ should be above 8, and LLG should be positive and as high as possible for successful molecular replacement [85]. Electron density maps and models were examined with Coot [33].

\subsubsection{Refinement}

Phenix.refine from the phenix program suite was used for KlAtg21-AgAtg16 and Rab33BAtg16L1 structure refinement [2]. For the AgAtg16 structure a first round of refinement by Refmac5 [94] from the CCP4 program suite was followed by phenix.refine. Initial cycles of rigid-body refinement were done for all structures followed by restrained refinement with alternating manual model building with Coot for the Rab33B-Atg16L1 and AgAtg16 structures. Rotamer and Ramachandran outliers of side chains and other geometric constraints were detected by phenix.refine output and manually corrected in Coot. Disagreements or increments with the model were identified by observation of the omit electron density map and correct by manual rebuilding in Coot. Coot ligand finder was used to place ligands in the Rab33B-Atg16L1 structure [33]. Refinement and manual corrections were repeated until the R-Factor and geometrical values were satisfying for the resolution limit of the structures.

\subsubsection{Generation of figures}

Figures were generated using the software Pymol [27]. 


\section{Results}

\subsection{Structural characterization of the murine Rab33B-Atg16L1 complex}

Rab33B was the first GTPase described to directly interact with an autophagy protein. The precise role of the Rab33B-Atg16L1 complex in autophagy needs further investigation. My goal was to determine the crystal structure of the Rab33B-Atg16L1 complex in order to understand how these proteins interact on a molecular level. This might help to understand how Golgi-derived vesicles are recruited to the phagophore. Itoh et al. [54] showed that that the Atg16L1 construct comprising residues 141-265 binds Rab33B and suggest that Atg16L1 is an effector of Rab33B. First steps for the characterization of the mRab33B Q92L mAtg16L1 complex were done before in our lab by Dr. Amanda Schalk [121], Michaela Hellwig and Dr. Karin Kühnel (personal communication). Dr. Amanda Schalk showed that full-length Rab33BQ92L is not soluble but the truncated Rab33B(30-202)Q92L is highly soluble and stable so this construct was used for expression [121]. Because Atg16L1 Rab33B interaction is GTP-dependent [54], Rab33B Q92L, a GTPase deficient mutant was used for in vitro experiments. A. Schalk purified the Rab33B(30-202)Q92L/ Atg5 Atg16L1(1-265) complex. However, attempts to crystallize this complex did not give crystals. With the goal to determine the structure of the Rab33B-Atg16L1 complex an optimal minimal construct of Atg16L1, which still binds Rab33B was tried to be identified. Fragments of the Atg16L1 coiled coil domain with variable length were tested for complex formation. Previous work by Dr. Amanda Schalk [121], Michaela Hellwig and Dr. Karin Kühnel showed that the minimal Atg16L1 binding region compromises residues 153-210, but the Atg16L1 construct 163-210 was not tested yet (Fig. 3.1.1.). 


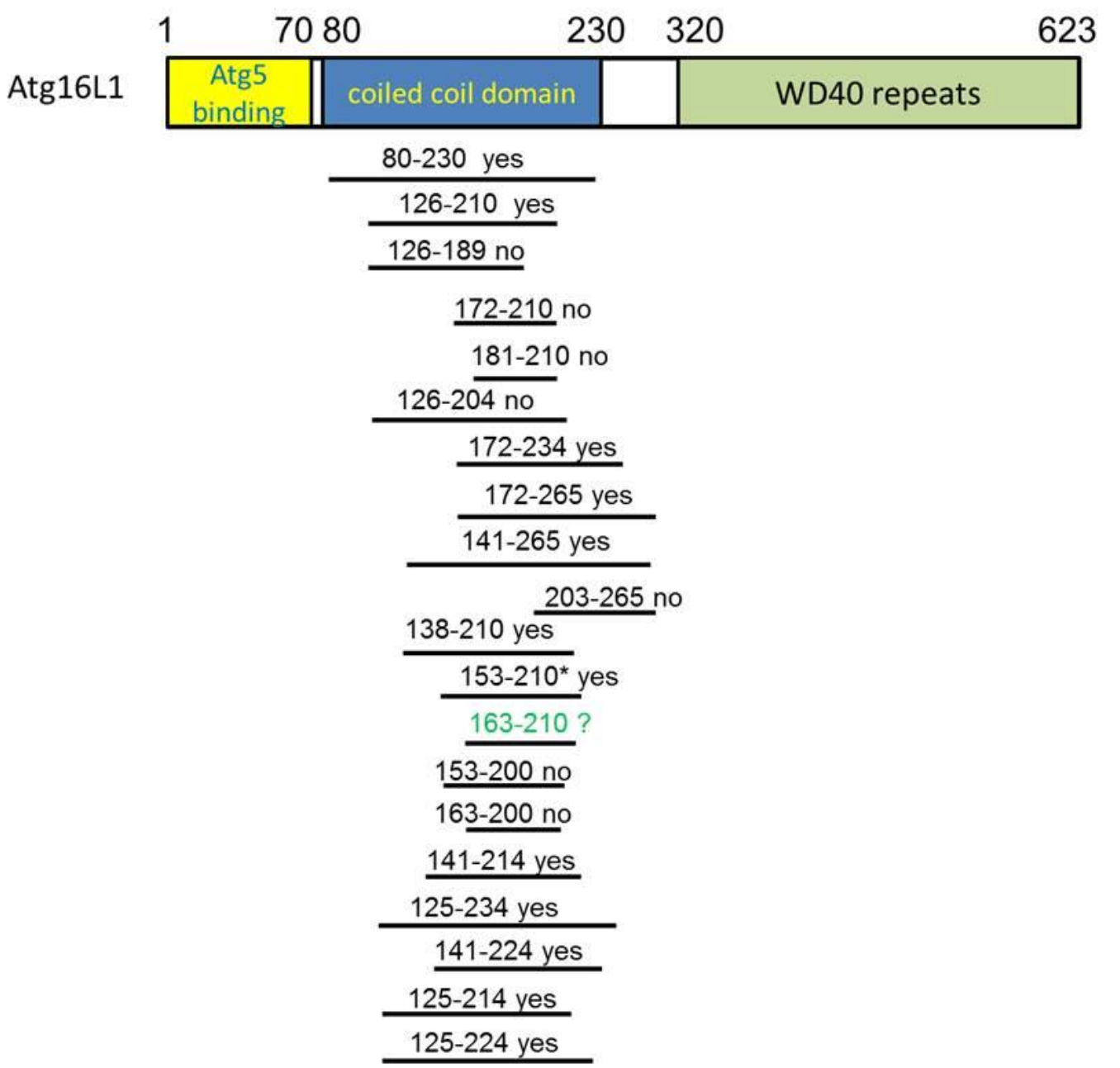

Figure 3.1.1.: Various truncated mAtg16L1 ccd constructs tested for Rab33B (30-202)Q92L binding. Yes or No indicates if complex formation was observed. Construct mAtg16L1(163-210) was cloned but not yet analyzed. (Modified figure from Dr. Karin Kühnel)

\subsubsection{Recombinant expression and purification of Rab33B, Atg16L1 and Rab33B- $\operatorname{Atg16L1}$ complexes}

For expression and purification of the complex the already available construct of mRab33Q92L(30-202) as an N-terminal His-tagged protein in the MCSI and untagged mAtg16L1 variants in the MCSII of the pETDuet-1 vector were used. For large scale purification the complex was purified by HisTrap affinity chromatography followed by a polishing step with size exclusion chromatography using a HiLoad 16/60Superdex 200 column (Fig. 3.1.2.). Schägger gel analysis shows that the mRab33B-mAtg16L1 complex eluted first from the gel filtration column followed by mRab33B alone (Fig. 3.1.2. D). Due to the small size of Atg16L1 and the large amounts of protein only one peak is visible in the 
chromatogram for Rab33B and Rab33B-Atg16L1 complex. Only fractions containing the complex were used for further experiments. The mRab33B(30-202)Q92L-mAtg16L1(153210) (Fig. 3.1.2.) and mRab33B(30-202)Q92L-mAtg16L1 (163-210) complex (Fig. 3.1.3.) were both purified, identifying mAtg16L1(163-210) as the minimal Atg16L1 coiled coil domain required for Rab33B binding. Both complexes were used for crystallization studies. The mRab33B-mAtg16L1(153-210) complex was more stable than the mRab33BmAtg16L1(163-210) and was used for all further studies.

A

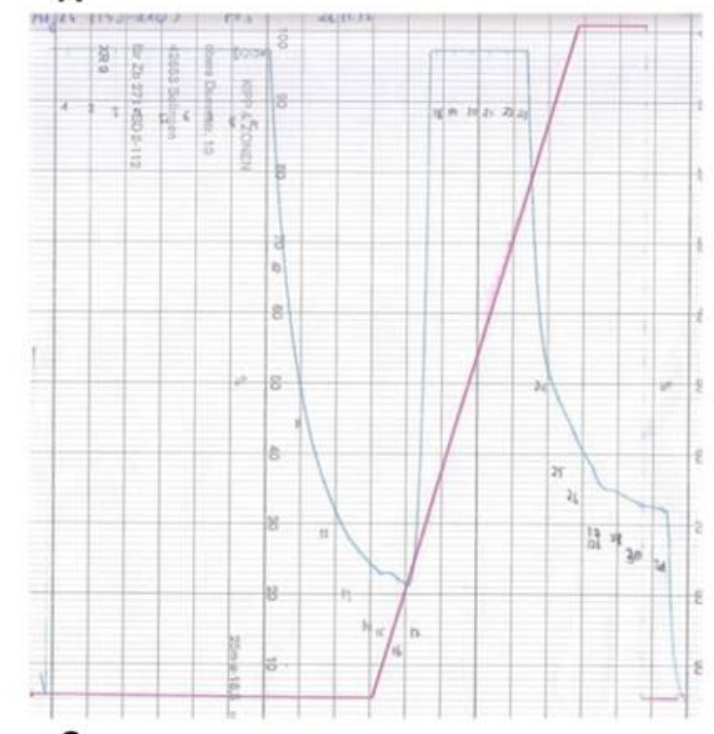

C

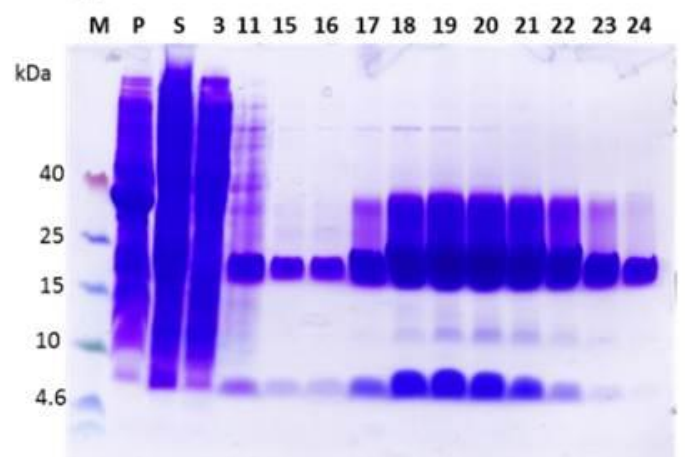

B

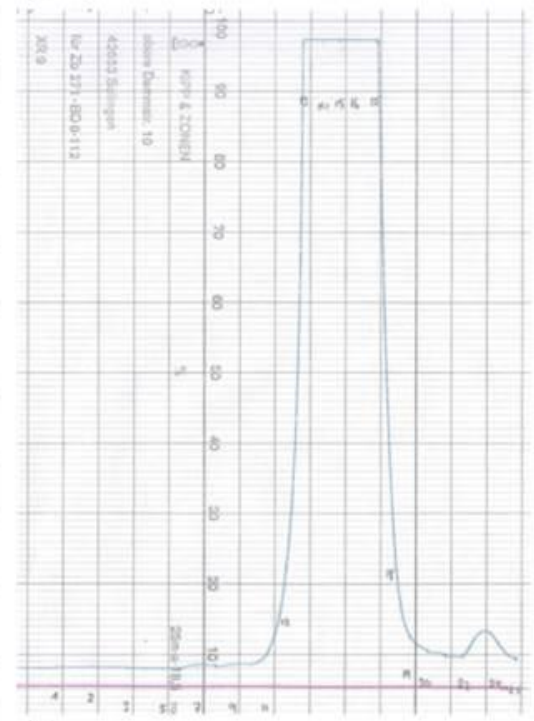

D

M Start $10 \quad 11 \quad 12 \quad 13 \quad 14 \quad 15 \quad 16 \quad 17 \quad 18 \quad 1920$

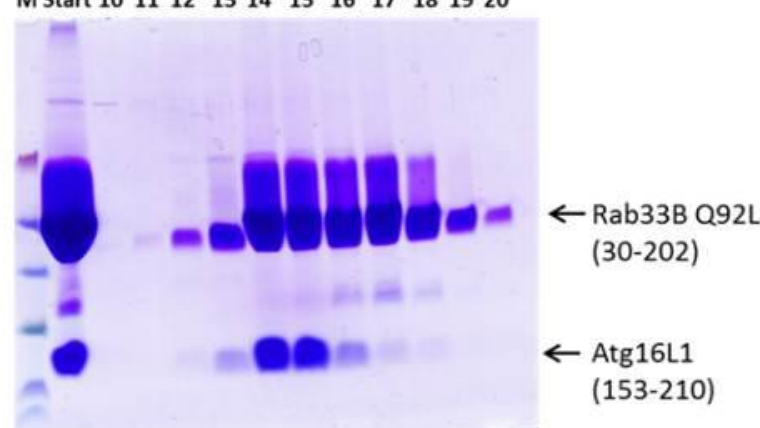

Figure 3.1.2.: Purification of the $\operatorname{Rab33B(30-202)Q92L-Atg16L1(153-210)}$ complex. Chromatograms of HisTrap affinity A: and size exclusion chromatography B: using a HiLoad 16/60 Superdex 200 column. C, D: Schägger gels of purification steps A and B, respectively. M: Marker, Start: Input for SEC. 
A
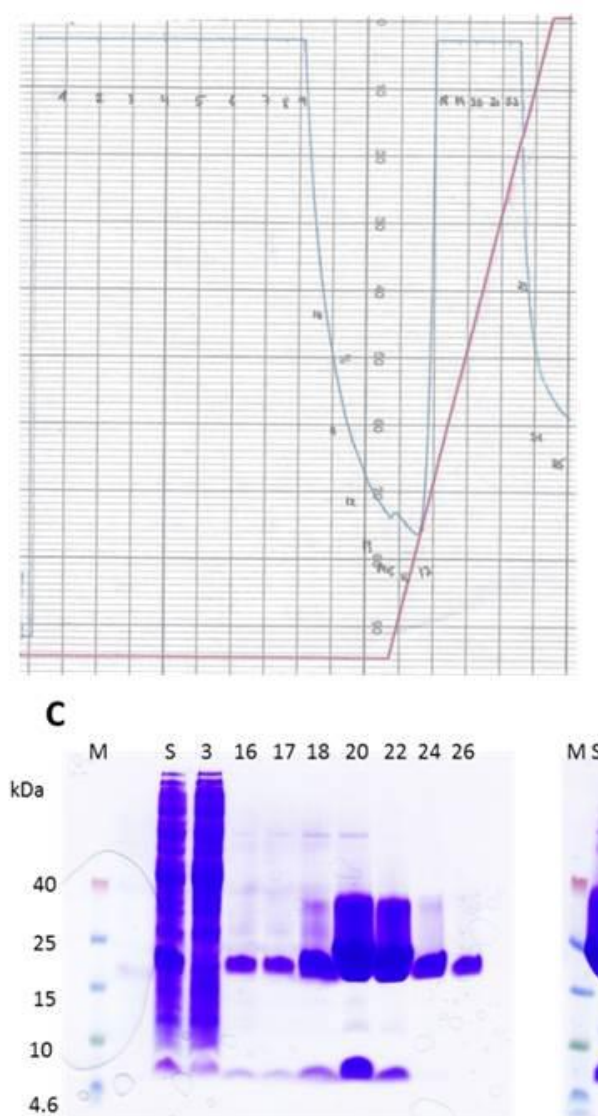

B

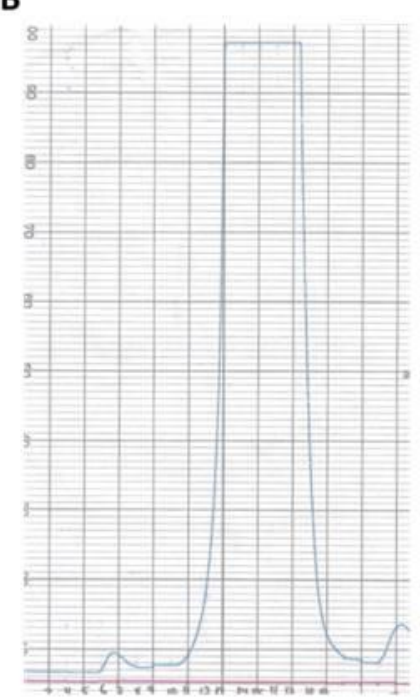

D

MStart $7 \quad 11 \quad 121314151617 \quad 1819 \quad 20$

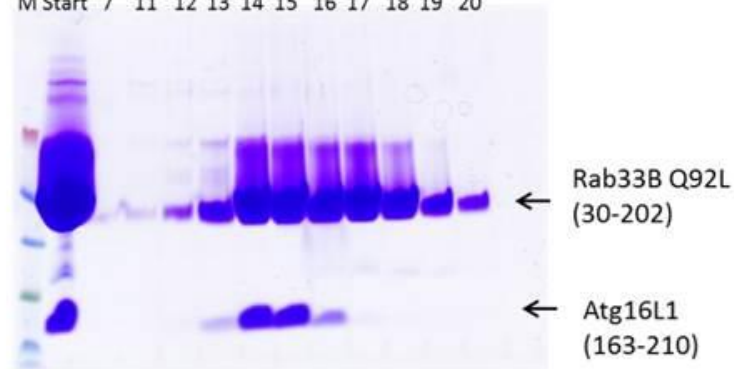

Figure 3.1.3.: Purification of the Rab33B(30-202)Q92L-Atg16L1(163-210) complex. Chromatograms of HisTrap affinity A: and size exclusion chromatography B: using a HiLoad 16/60 Superdex 200 column. C, D: Schägger gels of purification steps A and B, respectively. M: Marker, Start: Input for SEC.

For expression and purification of the individual proteins mRab33Q92L(30-202) was used as an N-terminal His-tagged protein in the MCSI of the pETDuet-1 vector. mAtg16L1(153-210) was cloned as an N-terminal His-tagged protein in the pET28b vector using full length mAtg16L1 as a template. Distinct proteins were purified as described before for the complex.

\subsubsection{Biochemical and biophysical characterization of Rab33B-Atg16L1 complex formation}

\subsubsection{ProteoPlex assay for the Rab33B-Atg16L1 complex}

With the aim to optimize buffer conditions and thereby enhance the chance for crystallizing the purified Rab33B(30-202)Q92L-mAtg16L1(153-210) complex was sent for an fluorescence-based thermal-shift assay (ProteoPlex) using the automated set-up in the laboratory of Prof. Dr. Holger Stark [22]. With this method one can analyze the influence of diverse factors like $\mathrm{pH}$, ionic strength and additives on the stability of a protein. 96 buffers 
were tested in an automated assay by Dr. David Haselbach (Department for Structural Dynamics). Results show that HEPES pH 7.4 buffer is among the most stabilizing buffers (Fig. 3.1.4.). An additive screen revealed that magnesium improved protein stability as well (data not shown), but this was not surprising because GTPases contain magnesium as a cofactor. Buffer conditions for subsequent purification were not changed, because a buffer consisting of $30 \mathrm{mM}$ HEPES $\mathrm{pH} 7.5,150 \mathrm{mM} \mathrm{NaCl}$ and $2 \mathrm{mM} \mathrm{MgCl}_{2}$ buffer was already being used.

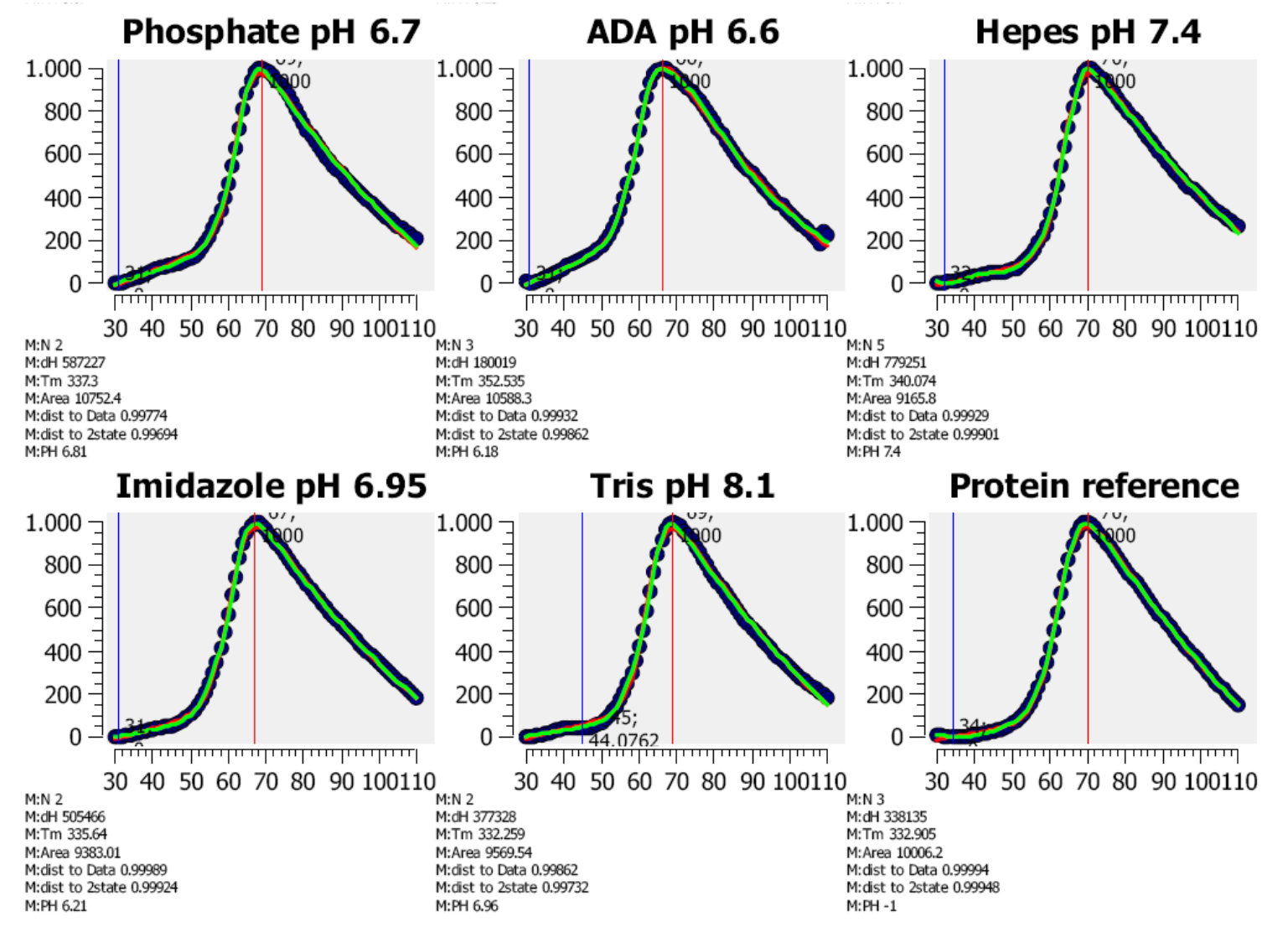

Figure 3.1.4.: ProteoPlex unfolding curves of the Rab33B(30-202)Q92L-Atg16L1(153-210) complex

\subsubsection{Limited proteolysis of the Rab33B-Atg16L1 complex}

Flexible domains and residues can hamper crystallization and limited proteolysis can be used to crop flexible elements and enhance crystallization. The mRab33(30-202)Q92LmAtg16L1(153-210) complex was digested with twelve different proteases from the ProtiAce Kit 1\&2 (Hampton Research) and DNaseI. DNaseI was chosen because purifications with too much DNaseI resulted in a partially degraded complex, possible due to a protease contamination in the DNaseI batch (Fig. 3.1.5.). 
A

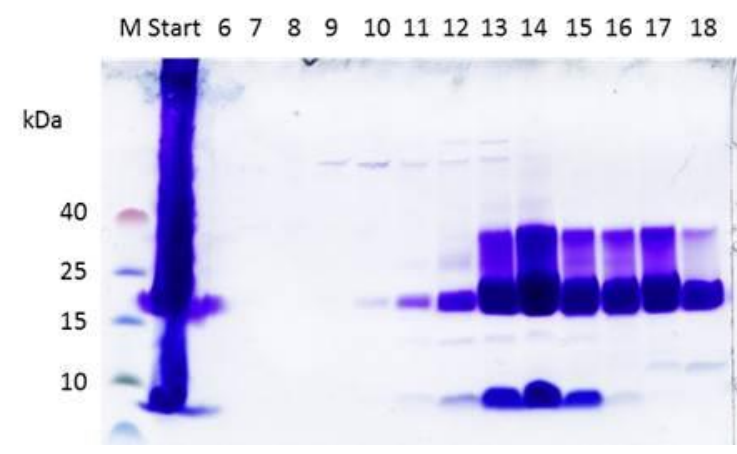

B

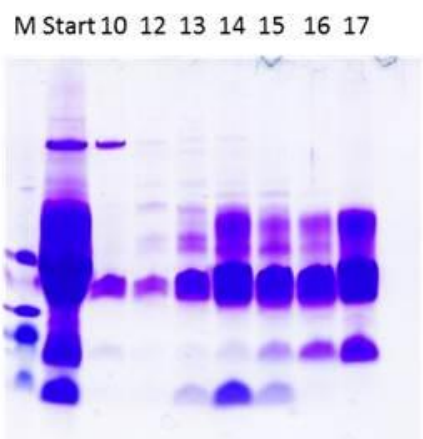

Figure 3.1.5.: Comparison of $\operatorname{Rab33B}(30-202) Q 92 L-A t g 16 L 1(153-210)$ complex purification with different DNaseI concentrations. Schägger gel electrophoresis of size exclusion chromatography using a HiLoad 16/60 Superdex 200 column. A: Cells were lysed with a small amount of DNaseI ( 1 small spatula tip/ pellet from 1.5L expression culture). B: Cells were lysed with a small amount of DNaseI ( 2 spatula tip/ pellet from 1.5L expression culture). M: Marker, Start: Input for SEC.

The purified complex $(4 \mathrm{mg} / \mathrm{ml})$ was mixed with 1:500 protease or DNaseI and incubated at $37{ }^{\circ} \mathrm{C}$. As a control the complex was incubated without protease. Samples were taken at different time points during protease incubation. Analysis by Schägger gels (Fig. 3.1.6.) showed that most proteases ( $\alpha-\mathrm{C}, \mathrm{TR}, \mathrm{P}-\mathrm{K}, \mathrm{CL}, \mathrm{EL}, \mathrm{PA}$ and SU) did not degrade the complex. Using Thrombin, PE, BR however resulted in the complete degradation of Atg16L1, whereas Rab33B remained mostly intact. Only for A-E and DNaseI after $60 \mathrm{~min}$ and EG-C after 30 min a promising partial digestion pattern was observed. For in-situ crystallization the Rab33(30-202)Q92L-Atg16L1(153-210) (45 mg/ml) complex was mixed with either 1:500 A-E, EG-C or DNaseI and incubated for $15 \mathrm{~min}$ at $37{ }^{\circ} \mathrm{C}$ prior to setting drops for crystallization. Digested proteins were screened in commercial crystallization screens but no crystallization hit was found. 


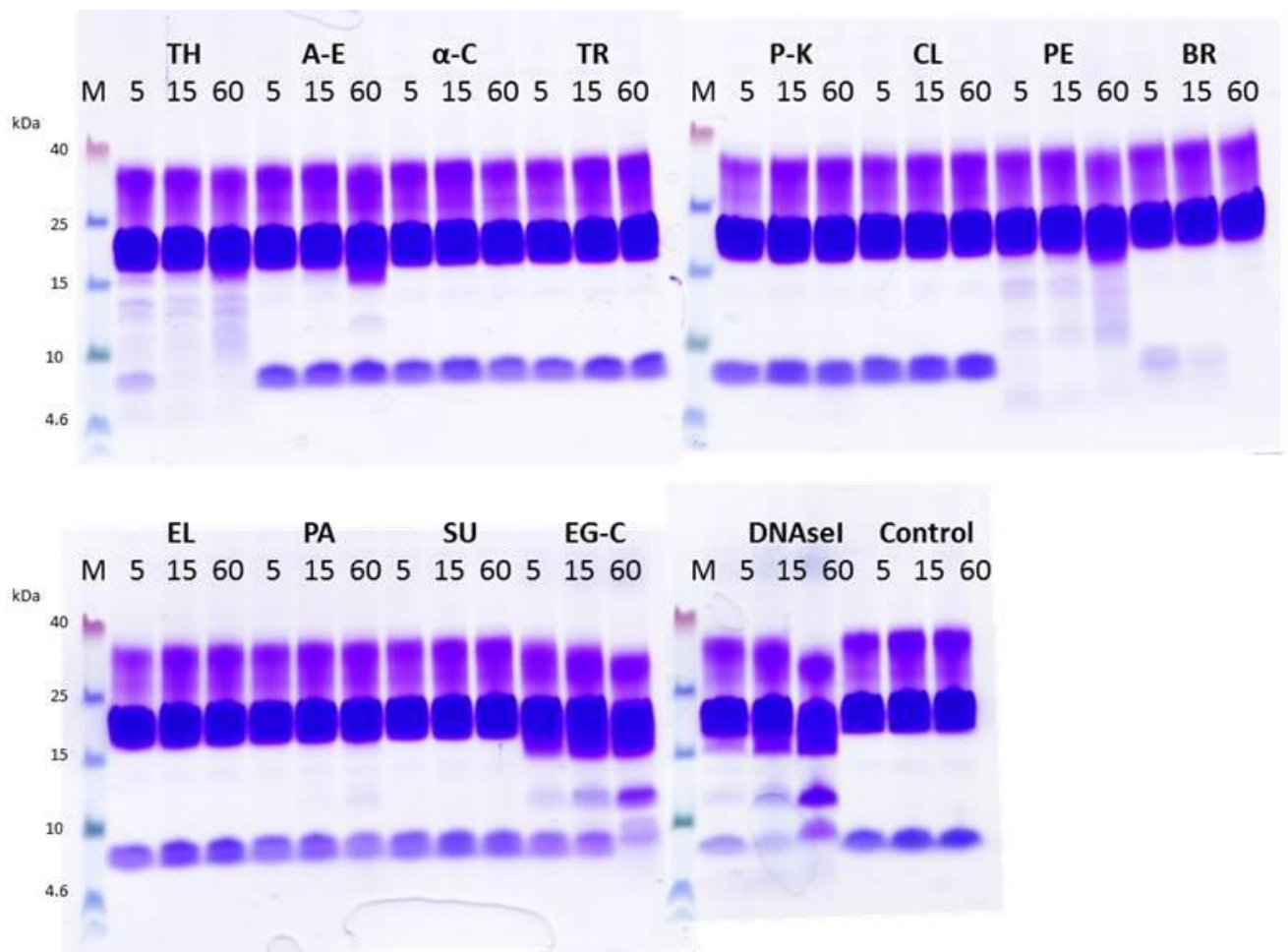

Figure 3.1.6.: Limited proteolysis of the Rab33B-Atg16L1 complex. Purified Rab33B(30202)Q92L-mAtg16L1(153-210) complex (4 mg/ml) was incubated with 1:500 proteases or DNaseI. Proteases used are TH: Thermolysin, A-E: Actinase, $\alpha-\mathrm{C}: \alpha$-Chymotrypsin, TR: Trypsin, P-K: Proteinase-K, CL: Clostripain, PE: Pepsin, BR: Bromelain, EL: Elastase, PA: Papain, SU: Substilisin, EG-C: Endoproteinase Glu-C. M: Marker. Numbers indicate incubation time at $37{ }^{\circ} \mathrm{C}$.

\subsubsection{Isothermal titration calorimetry measurements of the Rab33B-Atg16L1 complex} In order to characterize the thermodynamic parameters of the interaction between mRab33B(30-202)Q92L and mAtg16L1(153-210) isothermal titration calorimetry (ITC) measurements were conducted together with Dr. Ángel Pérez-Lara (Department of Neurobiology). $200 \mu \mathrm{M}$ mRab33B(30-202)Q92L was titrated in the cell containing $15 \mu \mathrm{M}$ mAtg16L1(153-210) (Fig. 3.1.7.). Binding is exothermic and proteins interact with high affinity with a $\mathrm{K}_{\mathrm{d}}$ of $0.21 \pm 0.02 \mu \mathrm{M}$. The stoichiometric analysis showed that two mRab33B(30-202)Q92L molecules bind to one Atg16L1 (153-210) dimer. 


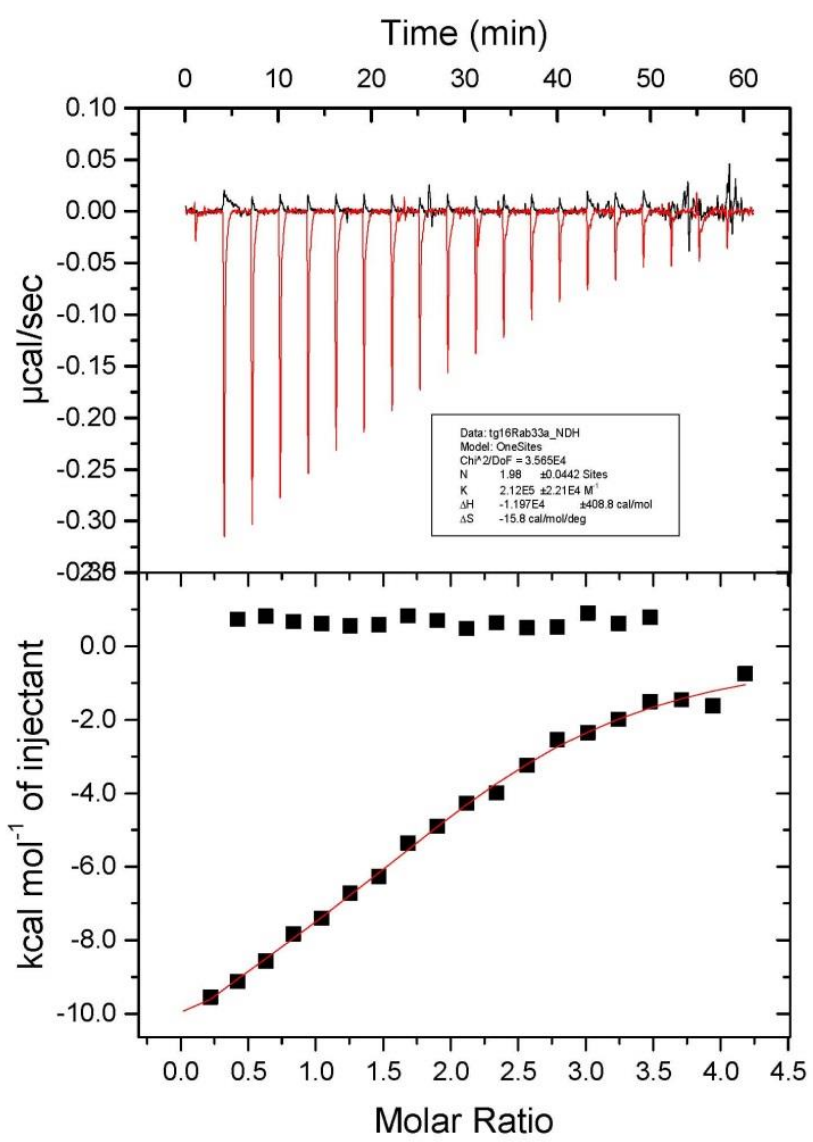

Figure 3.1.7.: Isothermal titration calorimetry titration curves of $m \operatorname{Rab33B}(30-202) Q 92 \mathrm{~L}$ and mAtg16L1(153-210). $200 \mu \mathrm{M}$ mRab33B(30-202)Q92L was titrated into $15 \mu \mathrm{M}$ mAtg16L1 (153-210). Data was fitted with a One Set of Sites fitting model. Top panel shows raw ITC data after subtraction of dilution enthalpies. Bottom panel shows integrated heat.

\subsubsection{Structure determination of the $\operatorname{Rab33B}(30-202) Q 92 L-A \operatorname{tg} 16 \operatorname{L1}(153-210)$ complex}

\subsubsection{Crystallization of the Rab33B-Atg16L1 complex}

With the aim of determining the $\mathrm{x}$-ray crystallographic structure of a mRab33B(30202)Q92L-mAtg16L1 complex commercial 96-well crystallization screens were set up using a pipetting robot and monitored with a Formulatrix imager. Both, mRab33B(30-202)Q92LmAtg16L1(153-210) and mRab33B(30-202)Q92L-mAtg16L1(163-210) were used for crystallization trials but the complex with the shorter Atg16L1 construct did not gave crystals. For mRab33B(30-202)Q92L-mAtg16L1(153-210) needle crystals were found initially after 510 days in a Qiagen Protein Complex 96-well screen. $60 \mathrm{nl}$ of $42 \mathrm{mg} / \mathrm{ml}$ protein and $60 \mathrm{nl}$ crystallization screen solution were pipetted in sitting drops and stored at $20{ }^{\circ} \mathrm{C}$ (Fig.3.1.8.). 

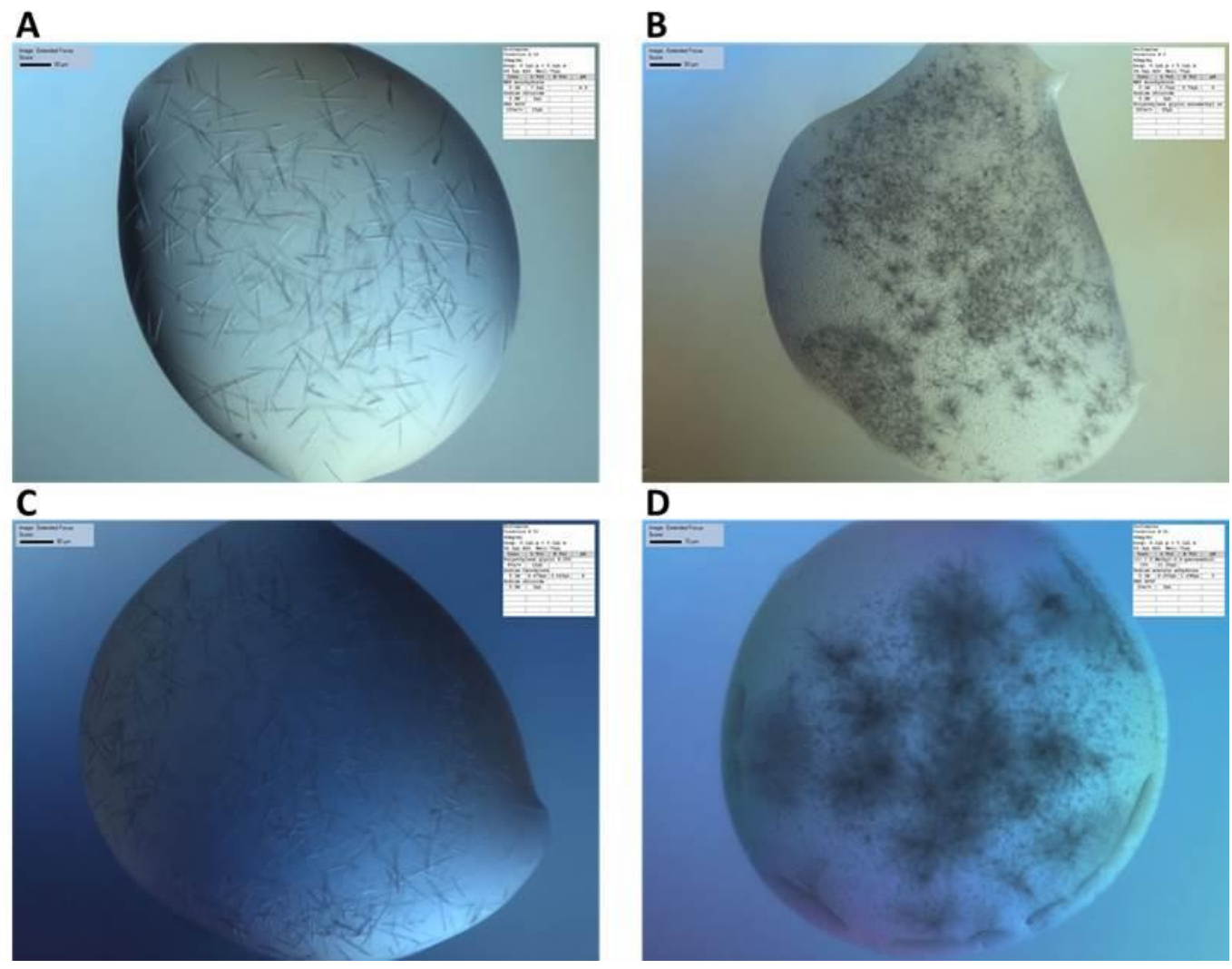

Figure 3.1.8.: Initial needle like crystals from Rab33B(30-202)Q92L-Atg16L1(153-210). Letters A-D correspond to the crystallization conditions stated in table 3.1.1.

Table 3.1.1.: Initial crystallization conditions for $\operatorname{Rab33B(30-202)Q92L-Atg16L1(153-210)~from~}$ 96-well plate screening

\begin{tabular}{|c|c|}
\hline Condition & Buffer composition \\
\hline A & $0.1 \mathrm{M}$ MES monohydrate $\mathrm{pH}$ 6.5, 0.2 M sodium chloride, $10 \%$ (w/v) PEG 4000 \\
\hline B & $0.1 \mathrm{M}$ MES monohydrate $\mathrm{pH}$ 6, $0.2 \mathrm{M}$ sodium chloride, $20 \%$ (w/v) PEG MME \\
\hline $\mathrm{C}$ & 0.1 M Sodium cacodylate $\mathrm{pH}$ 6, $0.2 \mathrm{M}$ sodium chloride, $8 \%$ (w/v) PEG 8000 \\
\hline $\mathrm{D}$ & $\begin{array}{l}0.1 \mathrm{M} \text { sodium acetate anhydrous } \mathrm{pH} 5,15 \% \text { (v/v) (+/-)-2-methyl-2,4-pentanediol, } \\
2 \% \text { (w/v) PEG } 4000\end{array}$ \\
\hline
\end{tabular}

Since crystals could not be reproduced on 24-well hanging drop plates optimization was also performed in 96-well sitting drops using the Gryphon 8-channel robot. Crystallization solutions from initial crystals were reproduced according to the manufacturer's protocol. $70 \mu \mathrm{l}$ of crystallization solution was pipetted in the reservoir of a 96-well plate and mixed with $7 \mu$ l of Hampton Additive Screen. An improvement was found using Qiagen ProteinComplex crystallization solution 91 with the addition of $0.1 \mathrm{M}$ Tris(2-carboxyethyl)phosphine (TCEP) hydrochloride (Fig. 3.1.9.). 


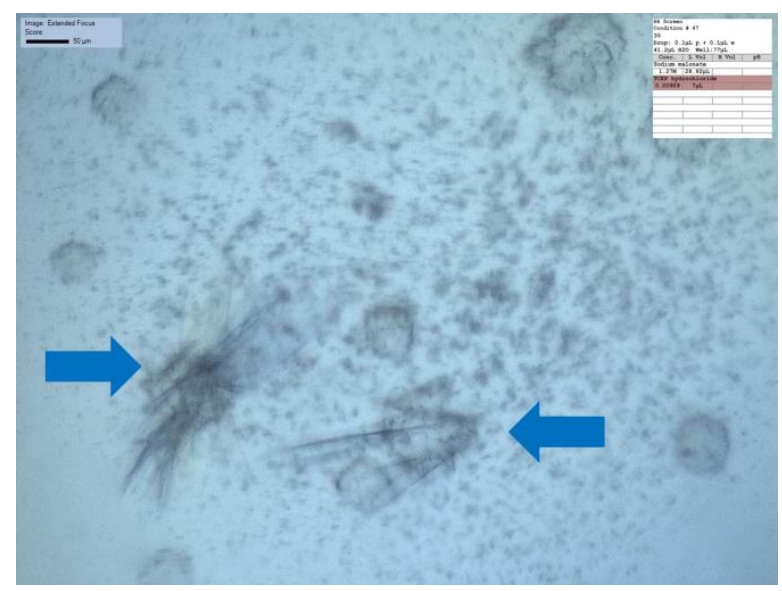

Figure 3.1.9.: Rab33B(30-202)Q92L-Atg16L1(153-210) crystals using the Hampton Additive Screen. Precipitant contained 0.1 M MES monohydrate pH 6.5, $0.2 \mathrm{M}$ sodium chloride, $10 \%(\mathrm{w} / \mathrm{v})$ PEG 4000, $10 \mathrm{mM}$ TCEP hydrochloride. Arrows indicate crystals. The scale bar corresponds to 50 $\mu \mathrm{m}$.

Because TCEP hydrochloride improved crystallization, $1 \mathrm{mM}$ TCEP hydrochloride was then added to all protein purification buffers. mRab33B(30-202)Q92L-mAtg16L1(153-210) was purified with buffers containing TCEP and used for further optimization attempts. Several additives were found that also enhanced crystallization. Crystals from these conditions were reproduced in 24 -well plates using $1.5 \mu$ l protein $+1.5 \mu$ l precipitant hanging drops. $\mathrm{pH}$ and precipitant concentrations for crystallization solution A (see Table 3.1.1.) were varied using a grid screen but only reproduction of the original commercial solution gave crystals. All further crystallization attempts were done with this solution. Crystals were equilibrated in the precipitant supplemented with $25 \%$ ethylene glycol and then fished out with a loop and flash cooled in liquid nitrogen. Crystals diffracted to $\sim 9 \AA$ at beamline PXII at the Swiss Light Source (Switzerland).

To ensure that mRab33B is bound to GTP and not GDP and to further optimize crystallization GTP $\gamma$ S (Guanosine 5'-O-[ $\gamma$-thio]triphosphate) an artificial non-hydrolyzable form of GTP was added to the lysed bacteria during protein purification and to the pooled size exclusion chromatography fractions. Unfrozen protein $(48 \mathrm{mg} / \mathrm{ml})$ was used for crystallization with optimized conditions in 24-well plates. Crystals were equilibrated in crystallization solution supplemented with $25 \%$ ethylene glycol and flash cooled in liquid nitrogen. Diffraction was tested at the Swiss Light Source (Switzerland) at beamline PXI and crystals diffracted to 3.5$4.5 \AA$. 


\subsubsection{X-ray data collection and processing for the $\operatorname{Rab33B}(30-202) Q 92 L-A \operatorname{tg} 16 L 1(153-$}

\section{0) complex}

Data for structure determination of the mRab33B(30-202)Q92L-mAtg16L1(153-210) were collected from a crystal grown in a 24-well hanging drop plate. Protein buffer consisted of $20 \mathrm{mM}$ HEPES pH 7.5, $150 \mathrm{mM} \mathrm{NaCl}, 2 \mathrm{mM} \mathrm{MgCl}_{2}, 1 \mathrm{mM}$ TCEP hydrochloride, $10 \mu \mathrm{M}$ GTP $\gamma$ S. The crystal grew in a drop composed of $3 \mu 1$ protein $(48 \mathrm{mg} / \mathrm{ml}), 2 \mu 1$ crystallization solution $\mathrm{A}$ and $0.5 \mu \mathrm{l} 1 \mathrm{M}$ spermine. Crystal was soaked in crystallization solution supplemented with $25 \%$ ethylene glycol (EG) before flash cooling in liquid nitrogen. Data were collected at $100 \mathrm{~K}$ at beamline X06SA (Swiss Light Source, Paul Scherrer Institute, Villigen, Switzerland). After mounting of the crystal a grid screen was performed to find the best diffracting region of the crystal (Fig. 3.1.10.) Four test shots were taken at the best position for indexing of the crystal unit cell and the optimal collection strategy was determined with go.com (local software written by Dr. M. Wang, SLS).

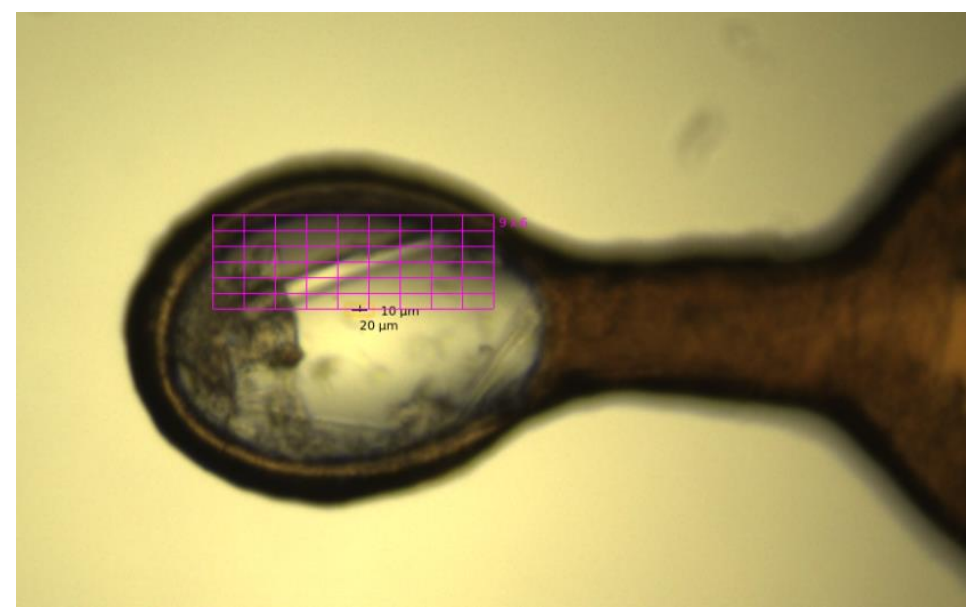

Figure 3.1.10.: Rab33B(30-202)Q92L-Atg16L1(153-210) crystal mounted at the Swiss Light Source. Pink squares define zone for grid screening. Each square has a size of 10 x $20 \mu \mathrm{m}$.

A complete native dataset with $360^{\circ}$ oscillation was collected at $1 \AA$ wavelength (for details see table 3.1.2.). Data were processed with input values specific for the EIGER 16M detector using the XDS software package. XDS defined the space group as monoclinic P12 1 and this was confirmed by the expected absences of reflections along the screw axis $(0,2 n, 0)$. The diffraction data were converted to unmerged CCP4F format with XDSCONV. 
Table 3.1.2.: Data collection for Rab33B(30-202)Q92L-Atg16L1(153-210) crystal

\begin{tabular}{|l|l|}
\hline Beamline & X06SA, Swiss Light Source (Switzerland) \\
\hline Detector & EIGER 16M (Dectris) \\
\hline Detector distance & $500 \mathrm{~mm}$ \\
\hline$\varphi / \Delta \varphi$ & $0.2 / 360^{\circ}$ \\
\hline Exposure time & $0.1 \mathrm{sec}$ \\
\hline Beam intensity & 0.1 \\
\hline$\lambda$ & $1 \AA$ \\
\hline Number of frames & 1800 \\
\hline Spacegroup & $\mathrm{P} 12_{1} 1$ \\
\hline
\end{tabular}

\subsubsection{Matthew's coefficient for the Rab33B-Atg16L1 complex}

The Matthew's coefficient [84] was calculated to estimate the number of macromolecules in the asymmetric unit. This number is important to search for the correct number of molecules during structure determination. The molecular weight of one molecule Rab33B(30-202)Q92L (20583 Da) and one molecule Atg16L1(153-210) (6779 Da) was combined giving $27632 \mathrm{Da}$ as input value for a 1:1 complex. The calculated Matthew's coefficient (Table 3.1.3.) did not gave a distinct value for molecules per asymmetric unit. 7, 8 or 9 molecules per asymmetric unit are most likely, corresponding to a water content of $43-56 \%$ in the crystal.

Table 3.1.3.: Matthews coefficient for $\operatorname{Rab33B}(30-202) Q 92 L-A \operatorname{tg} 16 \operatorname{L1}(153-210)$ crystals

For estimated molecular weight of $27362 \mathrm{Da}$.

Nmol/asym Matthews Coeff \% solvent $\quad \mathrm{P}(3.47) \quad \mathrm{P}($ tot $)$

\begin{tabular}{lllll}
\hline 1 & 19.41 & 93.67 & 0.00 & 0.00 \\
2 & 9.70 & 87.33 & 0.00 & 0.00 \\
3 & 6.47 & 81.00 & 0.00 & 0.00 \\
4 & 4.85 & 74.66 & 0.01 & 0.00 \\
5 & 3.88 & 68.33 & 0.03 & 0.02 \\
6 & 3.23 & 62.00 & 0.10 & 0.07 \\
7 & 2.77 & 55.66 & 0.22 & 0.19 \\
8 & 2.43 & 49.33 & 0.32 & 0.32 \\
9 & 2.16 & 42.99 & 0.24 & 0.29 \\
10 & 1.94 & 36.66 & 0.06 & 0.10 \\
11 & 1.76 & 30.33 & 0.00 & 0.01 \\
12 & 1.62 & 23.99 & 0.00 & 0.00 \\
13 & 1.49 & 17.66 & 0.00 & 0.00 \\
14 & 1.39 & 11.32 & 0.00 & 0.00 \\
15 & 1.29 & 4.99 & 0.00 & 0.00
\end{tabular}

\subsubsection{Molecular replacement for the Rab33B-mAtg16L1 complex}

The structure was determined by molecular replacement using the PHENIX program suite at $3.5 \AA$ resolution. The .mtz-file and a .fasta-file with the mRab33B(30-202)Q92L sequence were given as input for Phaser_MR, lacking the nucleotide and magnesium. The known 
structure of GppNHp-Bound Rab33 GTPase (PDB code: 1Z06) was used as search model. Analyzing the cell content did not give a clear indication of the number of molecules per asymmetric unit. Therefore, molecular replacement was tried with four to eight Rab33B molecules. The best solution was found for six Rab33B molecules per asymmetric unit (Table 3.1.4.). This solution revealed three additional regions with helical shape in the electron density map. The complete structure was determined by a second round of molecular replacement using the coiled coil domain of ScAtg16 (PDB code: 3A7O) as a search model. The coiled coil domain of mammalian and yeast Atg16 is evolutionary conserved (Fig. 3.1.11.). Heptad positions are identical in the coiled coil domain of lower and higher eukaryotes. Three Atg16 dimers were found in the asymmetric unit (Table 3.1.5.). Additional electron density was observed in switch I and II regions of Rab33B. GTP and $\mathrm{Mg}^{2+}$ were found in all six Rab33B molecules using Coot Ligand Finder giving further proof for the correctness of the molecular replacement solution.

Table 3.1.4.: Results of first round of molecular replacement with Phaser_MR for the Rab33BAtg16L1 complex structure. Using 1 Z06 as a search model for Rab33B.

\begin{tabular}{|c|c|c|c|}
\hline Component copies & Number of MR solutions & Top LLG & Top TFZ \\
\hline 4 & 12 & 2001.339 & 5.8 \\
\hline 5 & 1 & 1539.257 & 28 \\
\hline 6 & 1 & 2236.042 & 34.2 \\
\hline 7 & 6 & 2174.428 & 6.2 \\
\hline 8 & 6 & 2118.417 & 5.9 \\
\hline
\end{tabular}

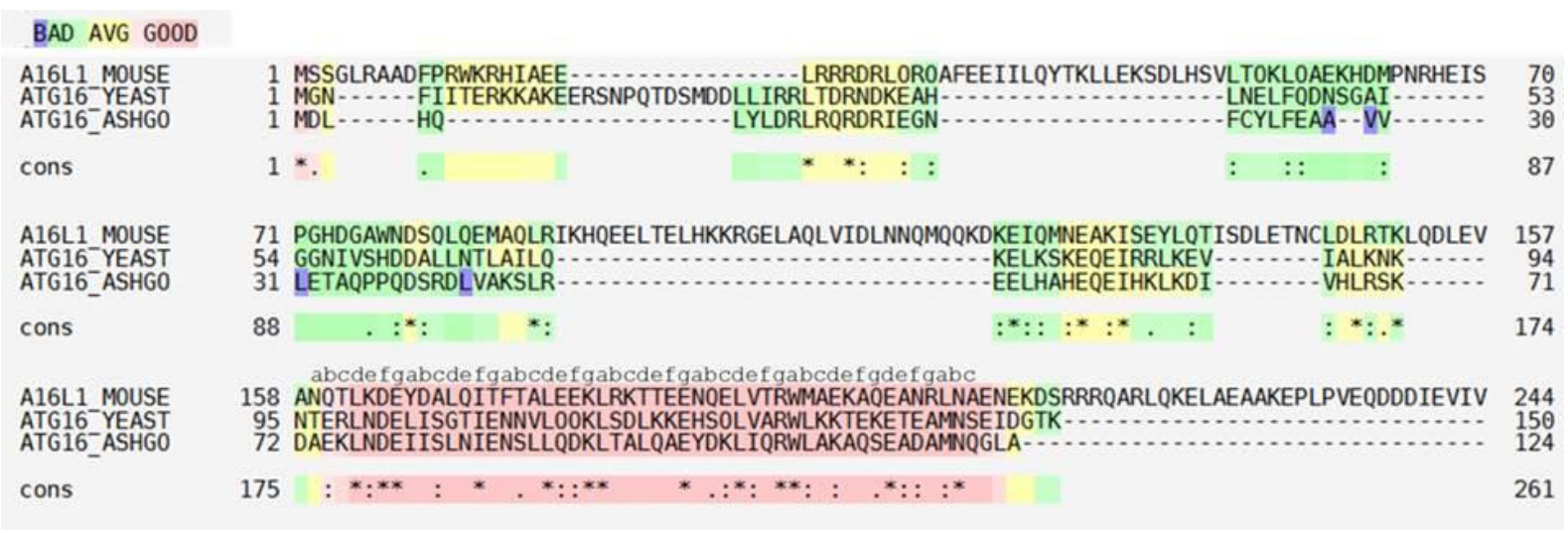

Figure 3.1.11.: Sequence alignment of yeast and mouse Atg16. Alignment was done with a structure based sequence alignment using T-Coffee Expresso [5]. Coiled coil heptad positions were assigned with TWISTER [130]. Alignment was truncated and the WD40 repeat of Atg16L1, not present in the yeast homologues is not shown. Colors indicate fitting score from blue (bad) to red (good). 
Table 3.1.5.: Result of second round of molecular replacement with Phaser_MR for the Rab33BAtg16L1 complex structure. Using six Rab33B molecules (Table 3.1.4.) as fixed partial solution and yeast Atg16 ccd 3A7O as a search model for Atg16L1.

\begin{tabular}{|c|c|c|c|}
\hline Component copies & Number of MR solutions & Top LLG & Top TFZ \\
\hline 3 & 1 & 2457.713 & 10.5 \\
\hline
\end{tabular}

\subsubsection{Refinement and structure validation for the Rab33B-Atg16L1 complex}

The initial model contained six Rab33B molecules and three Atg16L1 dimers. Manual model building was done with Coot and included rebuilding of Atg16L1 residues, addition of residues at the $\mathrm{C}$ - and $\mathrm{N}$-termini and side chain corrections. Rotamer outliers of side chains and geometric constraints were checked and corrected with Coot. Disordered side chains were deleted and residues were modelled as alanines (Table 3.1.6.). Manual model building and several cycles of refinement with Phenix were performed and resulted in the final structure with a $\mathrm{R}_{\text {work }} / \mathrm{R}_{\text {free }}$ of 20/24.6\%. Detailed data refinement statistics are listed in Table 3.1.7. The structure was validated using Phenix Polygon plot that compares the refinement statistics to structures in the PDB with a similar resolution cut-off (Fig. 3.1.12. A). All values lie within the permitted range. The Ramachandran plot was used to analyze the geometry of the model (Fig. 3.1.12. B). $95 \%$ of the residues are within the preferred regions, $4.6 \%$ in the allowed regions and no outliers were found.

Table 3.1.6.: Rab33B(30-202)Q92L-Atg16L1(153-210) residues modeled as alanine in the crystal structure.

\begin{tabular}{|c|c|c|c|}
\hline Chain & Residues & Chain & Residues \\
\hline A; mRab33B & R61, K97, L137, N139 & I; mAtg16L1 & $\begin{array}{c}\text { N159, K163, E197, } \\
\text { K198 }\end{array}$ \\
\hline B; mRab33B & $\begin{array}{c}\text { E63, K97, N181, N139, } \\
\text { R153, K165 }\end{array}$ & J; mAtg16L1 & K163, R181 \\
& R61, K133, L137, D184, & K; mAtg16L1 & K163, E197 \\
\hline C; mRab33B & K57, R61, E63, D69, & L; mAtg16L1 & K163, D167 \\
\hline D; mRab33B & K133, D140, K165, & & \\
& N185, H187, K198, L199, & & \\
& K200 & & N159, Q160, K163, \\
& D69, E80, R81, K97, & M; mAtg16L1 & \\
\hline E; mRab33B & K133, N183, D184, & & Q160, K163 \\
& K198, K200 & & \\
\hline F; mRab33B & R61, D69, K97, K133, & N; mAtg16L1 & \\
& N181, D186 & & \\
\hline
\end{tabular}


Table 3.1.7.: Data collection and refinement statistics of $\operatorname{Rab33B}(30-202)$ Q92L-Atg16L1(153210) complex. Values in parentheses refer to the highest resolution shell, Ramachandran statistics were calculated with Molprobity.

\begin{tabular}{|c|c|}
\hline $\begin{array}{l}\text { Data collection } \\
\text { Space group }\end{array}$ & $\mathrm{P} 12{ }_{1} 1$ \\
\hline $\begin{array}{l}\text { Cell dimensions } \\
\mathrm{a}, \mathrm{b}, \mathrm{c}(\AA) \\
\alpha, \beta, \gamma\left({ }^{\circ}\right) \\
\end{array}$ & $\begin{array}{r}48.4,204.9,107.2 \\
90.0, \quad 92.6, \quad 90.0 \\
\end{array}$ \\
\hline $\begin{array}{l}\text { Resolution range }(\AA) \\
\text { Total reflections } \\
\text { Unique reflections } \\
\text { Multiplicity } \\
\text { Completeness }(\%) \\
\text { Mean } \mathrm{I} / \sigma \\
\text { Wilson B factor }\left(\AA^{2}\right) \\
\mathrm{R}_{\text {meas }}(\%) \\
\mathrm{CC}_{1 / 2}\end{array}$ & $\begin{array}{l}47.06-3.47(3.59-3.47) \\
90400(6535) \\
26081(2005) \\
3.5 \\
96.4(78.3) \\
7.9(2.1) \\
66.5 \\
22.9(91.2) \\
98.3(70.7)\end{array}$ \\
\hline $\begin{array}{l}\text { Refinement } \\
\mathrm{R}_{\text {work }} \\
\mathrm{R}_{\text {free }} \\
\end{array}$ & $\begin{array}{l}0.203(0.326) \\
0.246(0.37)\end{array}$ \\
\hline $\begin{array}{l}\text { Molecules/AU } \\
\text { Number of protein residues included in model: }\end{array}$ & $\begin{array}{l}12 \\
\text { A: } 31-202 \\
\text { B: } 31-183,187-202 \\
\text { C: } 31-202 \\
\text { D: } 31-136,140-181,185-202 \\
\text { E: } 30-202 \\
\text { F: } 30-202 \\
\text { I: } 159-208 \\
\text { J: } 160-208 \\
\text { K: } 159-208 \\
\text { L: } 159-208 \\
\text { M: } 160-208 \\
\text { N: } 160-208\end{array}$ \\
\hline $\begin{array}{l}\text { Number of non-hydrogen atoms } \\
\text { Macromolecules } \\
\text { Ligands }\end{array}$ & $\begin{array}{l}10590 \\
10392 \\
198 \\
\end{array}$ \\
\hline $\begin{array}{l}\text { B-factors }\left(\AA^{2}\right) \\
\text { Macromolecules } \\
\text { Ligands } \\
\text { Structure validation } \\
\text { Ramachandran favored }(\%) \\
\text { Ramachandran allowed }(\%) \\
\text { Ramachandran outliers }(\%) \\
\text { Rotamer outliers }(\%) \\
\text { Clashscore }\end{array}$ & $\begin{array}{l}57.0 \\
57.3 \\
41.1 \\
\\
95 \\
4.6 \\
0 \\
0.46 \\
4.60\end{array}$ \\
\hline $\begin{array}{l}\text { RMSD deviations } \\
\text { Bond lengths }(\AA) \\
\text { Bond angles }\left({ }^{\circ}\right)\end{array}$ & $\begin{array}{l}0.005 \\
0.70\end{array}$ \\
\hline
\end{tabular}


A

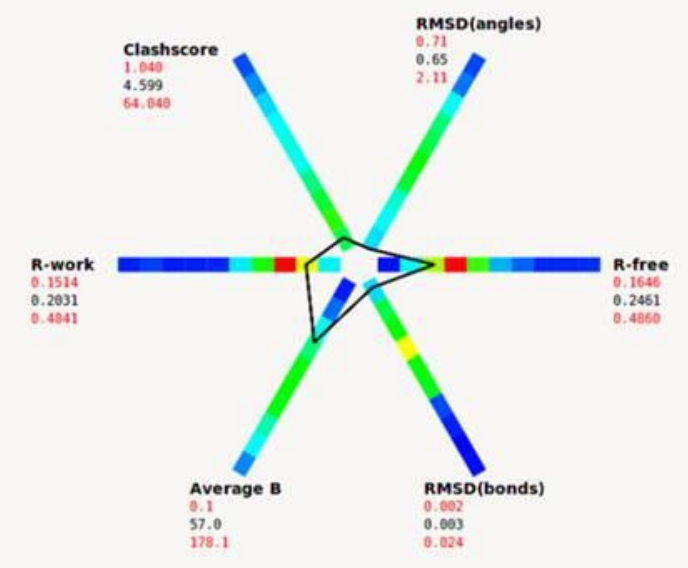

B

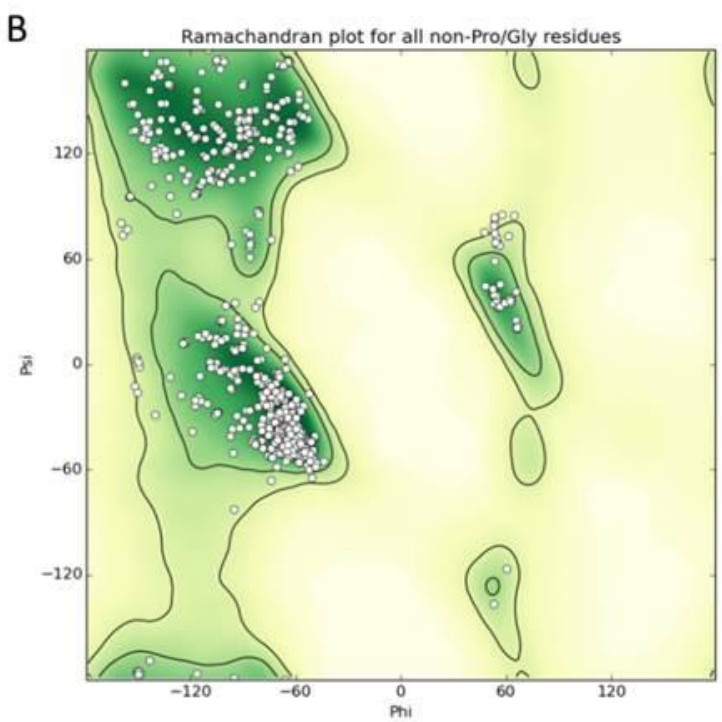

Figure 3.1.12.: Refinement statistic plots for the Rab33B(30-202)Q92L-Atg16L1(153-210) structure. A: Polyglon plot. Structure statistics (black numbers) are compared to PDB entries with similar resolution (red numbers). B: Ramachandran plot of all non Pro/Gly residues. Plots were made by Phenix refine. 


\subsubsection{Structure analysis for the Rab33B-Atg16L1 complex}

In total twelve molecules are observed in the asymmetric unit of the crystal structure (Fig.3.1.13). They form three mRab33B(30-202)Q92L-mAtg16L1(153-210) complexes. Two mRab33 molecules form a complex with the C-terminal region of a parallel mAtg16L1 dimer (Fig.3.1.14).

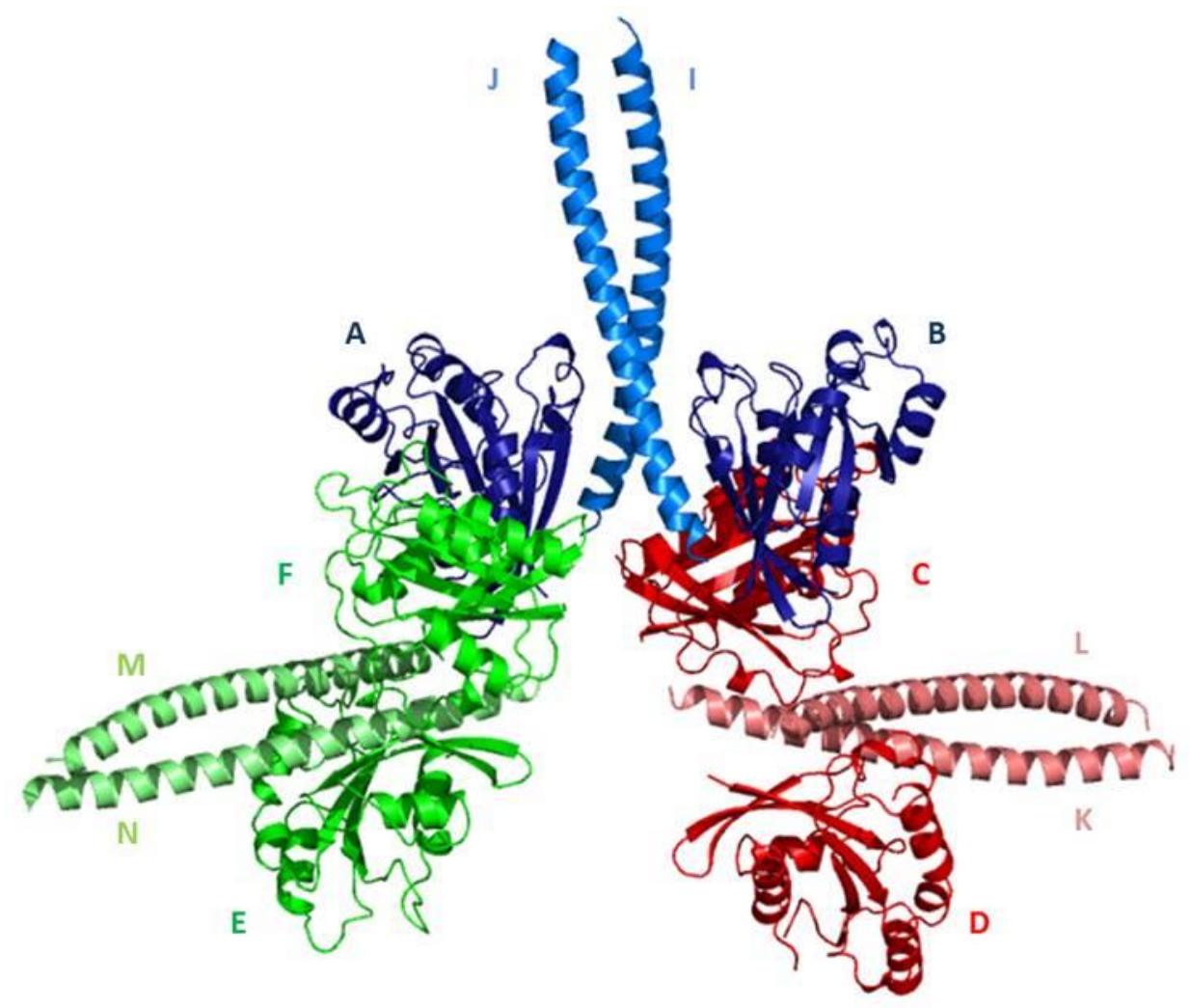

Figure 3.1.13.: Three $\operatorname{Rab33B(30-202)Q92L-Atg16L1(153-210)~complexes~are~present~in~the~}$ asymmetric unit. Two mRab33 molecules interact with one mAtg16L1 dimer. Rab33B molecules A and $\mathrm{B}$ are colored blue, $\mathrm{C}$ and $\mathrm{D}$ red, $\mathrm{E}$ and $\mathrm{F}$ green. $\mathrm{mAtg} 16 \mathrm{~L} 1$ dimer composed of chains $\mathrm{I}$ and $\mathrm{J}$ is colored light blue, the KL dimer light red and the MN dimer light green. 
A

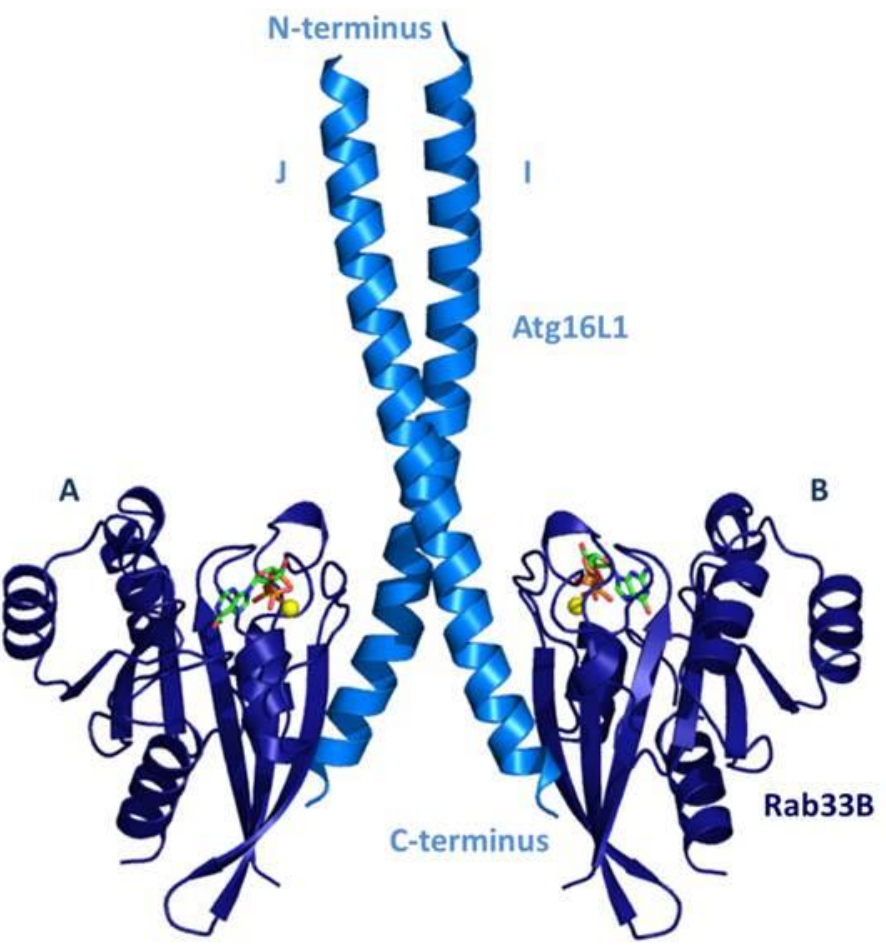

B

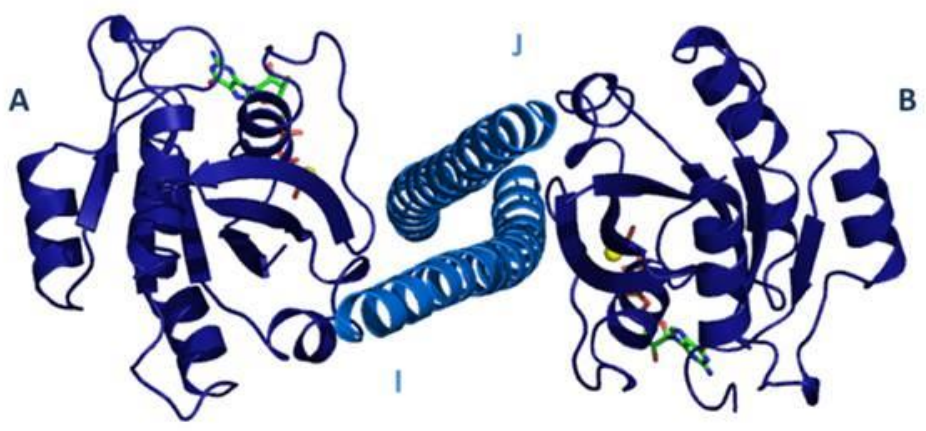

Figure 3.1.14.: Structure of the $\operatorname{Rab33B}(30-202) Q 92 L-A \operatorname{tg} 16 \operatorname{L1}(153-210)$ complex. The complex consists of two Rab33B molecules that bind to the diverging C-termini of the Atg16L1 dimer respectively. The Atg16L1 binding site is nearby the Rab33 GTP binding site. Rab33B (dark blue) and Atg16L1 (light blue) are in cartoon representation. The GTP as sticks in green and orange and the $\mathrm{Mg}^{2+}$ ion as yellow sphere.

Rab33B chains $\mathrm{A} / \mathrm{F}$ and $\mathrm{B} / \mathrm{C}$ are very close due to crystal packing. Crystal packing is loose and there are large solvent filled channels between symmetry-related molecules (Fig. 3.2.15). The three individual complexes align with a root-mean-square deviation of atomic positions (RMSD) of 0.602. Rab33B molecules are quite similar, whereas the Atg16L1 molecules are slightly shifted in the N-terminus region (Fig. 3.2.16.). 


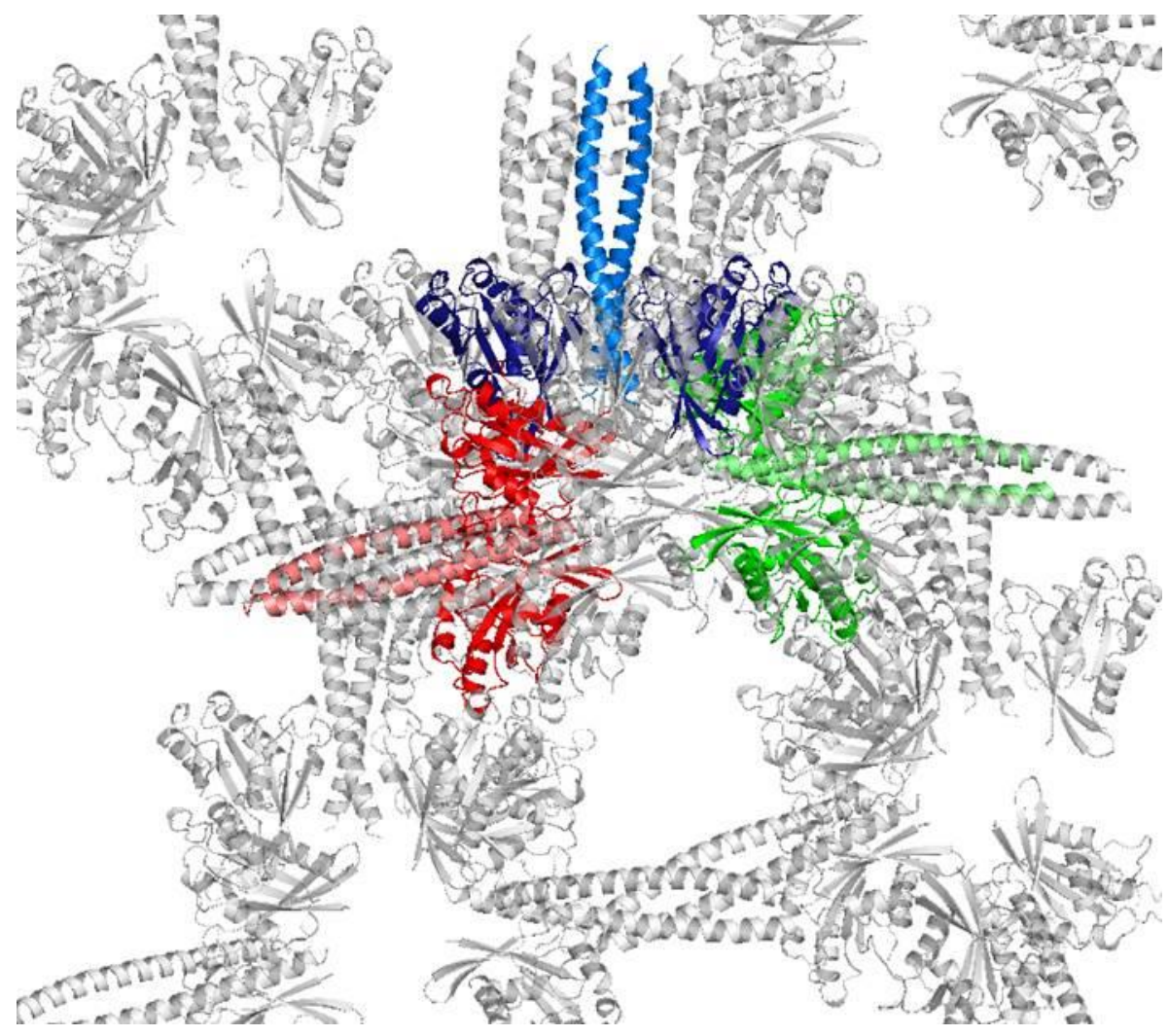

Figure 3.1.15.: Crystal packing of the Rab33B(30-202)Q92L-Atg16L1(153-210) crystal. Twelve molecules forming three Rab33-Atg16L1 complexes are present in the asymmetric unit are shown in color and symmetry-related molecules are colored grey.

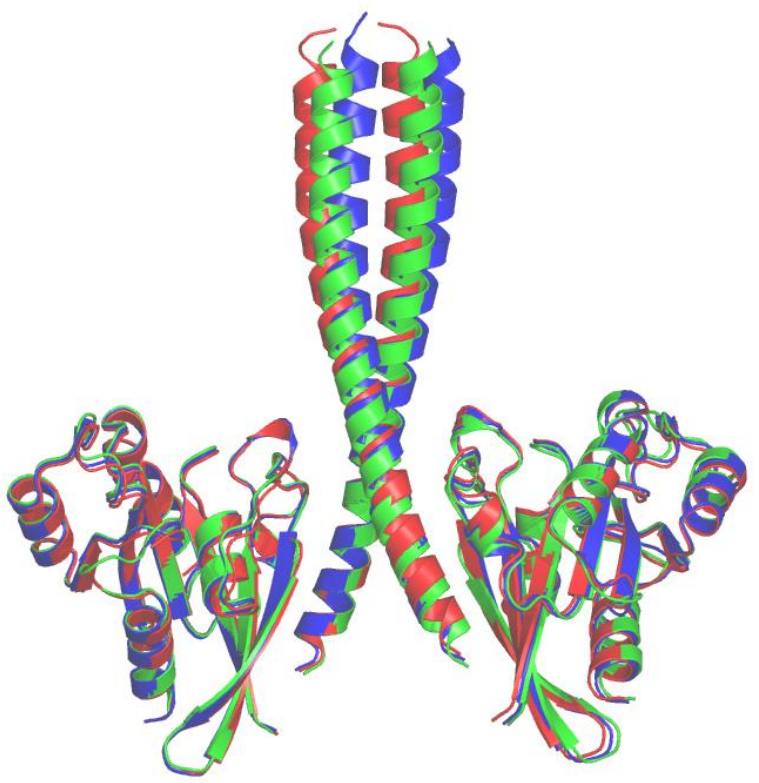

Figure 3.1.16.: Alignment of the three Rab33B(30-202)Q92L-Atg16L1(153-210) complexes found in the asymmetric unit. Cartoon representation of complexes ABIJ in blue, CDKL in red and EFMN in green. The complexes align with a RMSD of 0.602 
The Atg16L1 bound Rab33B structure is very similar to the known Rab33B structures [31, 103] mRab33B(30-202)Q92L exhibits the typical GTPase fold (Fig. 3.1.17 A). It consists of a central six-stranded $\beta$-sheet made of five parallel strands and an antiparallel strand, surrounded by five $\alpha$-helices. The GTPase fold is conserved for Rab proteins (Fig. 3.1.18. and Fig. 3.1.19.) Binding of Atg16L1 takes place at switch I, switch II and the interswitch region (Fig. 3.1.17. B). Ligands GTP and $\mathrm{Mg}^{2+}$ bind as expected in the switch I and II regions and the $5^{\prime}$-posphate arm of GTP is flanked by the P-loop (Walker A motif) (Fig. 3.1.17 A). Binding of GTP and $\mathrm{Mg}^{2+}$ is consistent with ligand binding observed in other GTP bound Rab crystal structures (Fig. 3.1.18.).
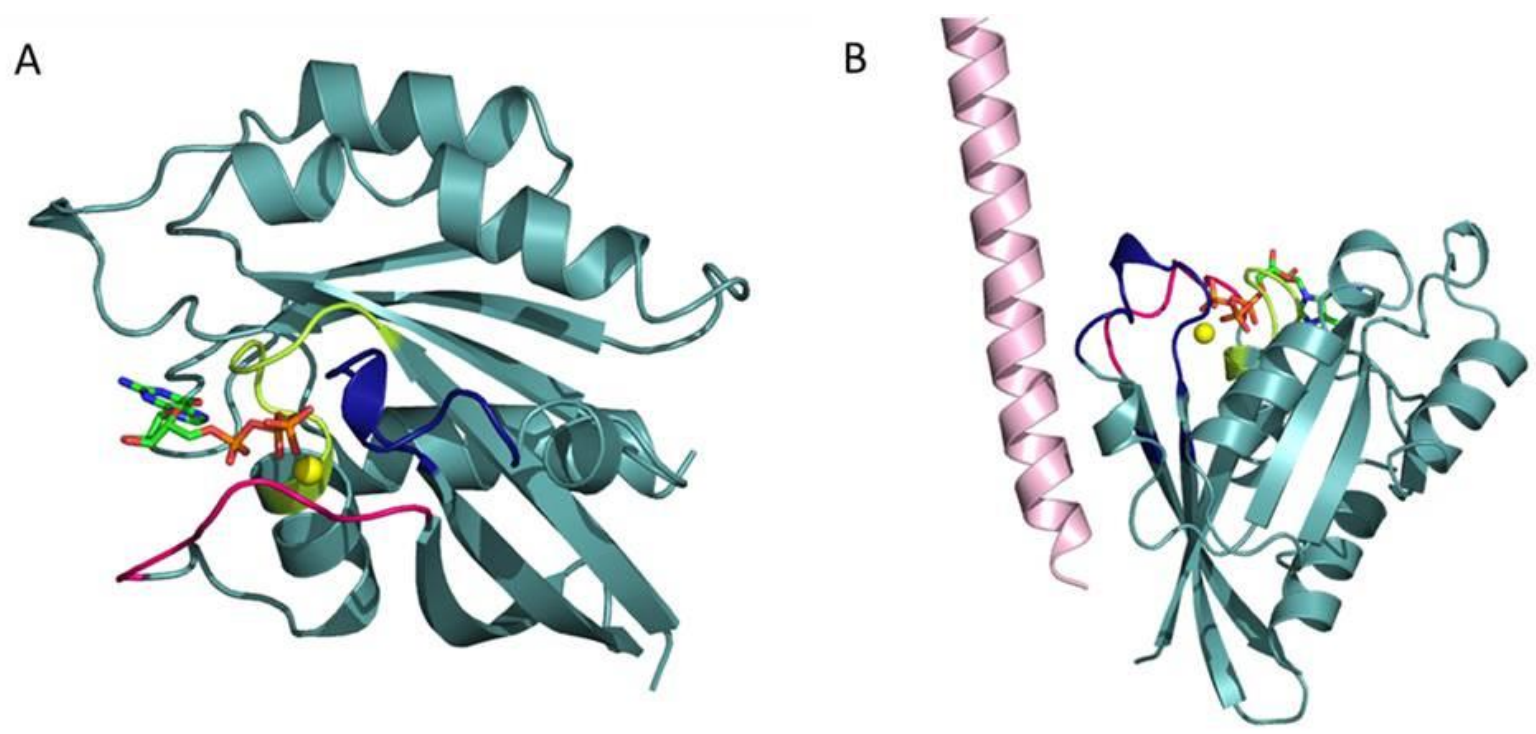

Figure 3.1.17.: Typical GTPase fold of $\operatorname{Rab33B(30-202)Q92L.~A:~Overview~of~Rab33B~structure.~}$ Cartoon representation of mRab33B in dark turquoise. Bound ligands GTP is shown as a stick model and magnesium as a yellow sphere. Switch I is colored pink, Switch II dark blue and the P loop lemon. B: Details of Atg16L1 binding site. Atg16L1 chain is colored in light pink. 


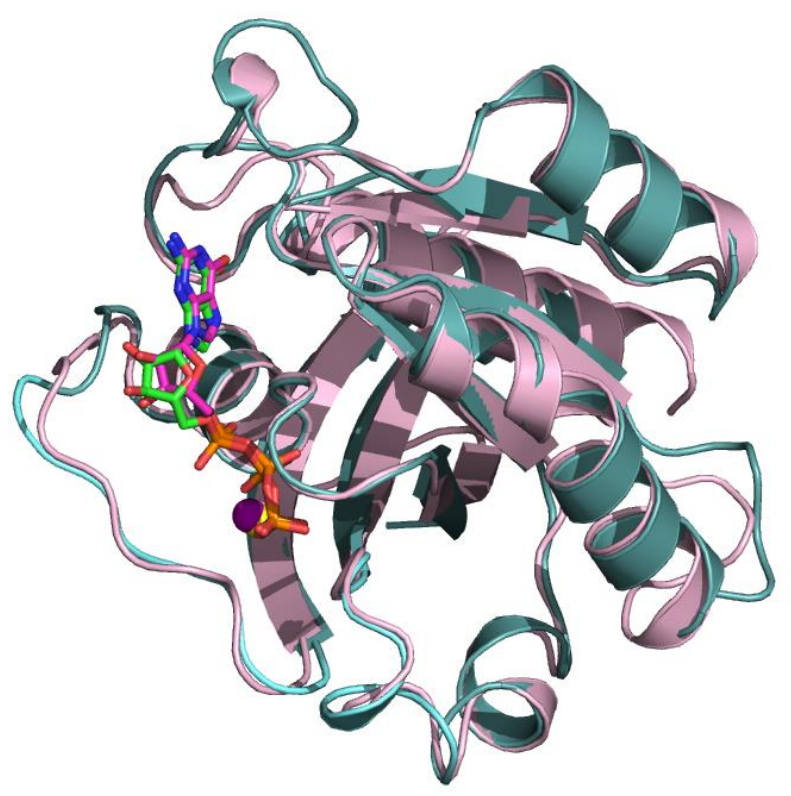

Figure 3.1.18.: Overlay of GTP bound Rab33B and Rab1a structures. Cartoon representation of mRab33B in dark turquoise with GTP shown as green carbon stick model and magnesium as a yellow sphere superimposed with Rab1a (PDB code 3TKL) in light pink with bound ligands GTP as pink carbon stick model and magnesium as pink sphere.
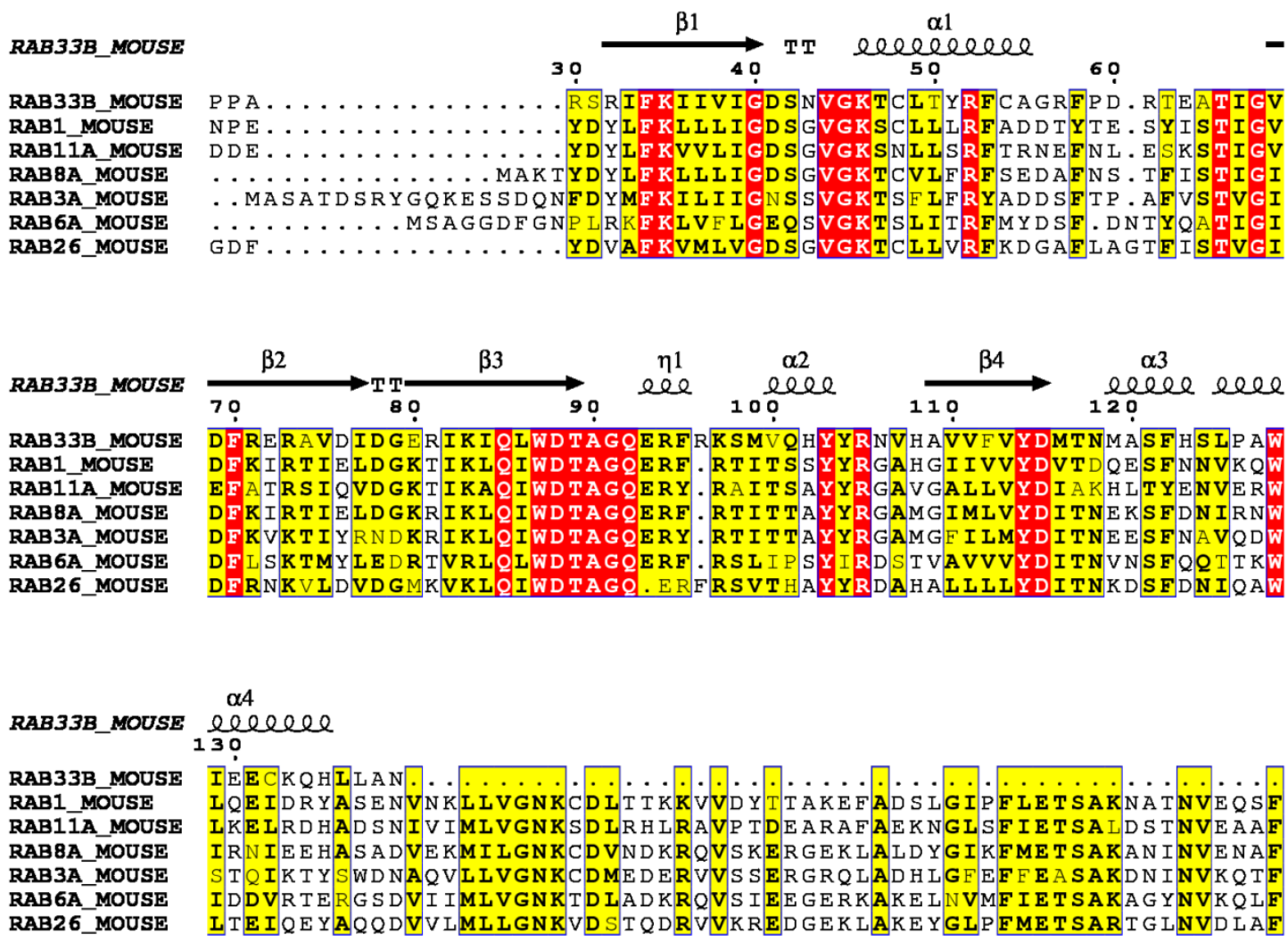

Figure 3.1.19.: Sequence alignment of murine Rab proteins. Arrows and helices on top of the alignment present structural elements of Rab33B. Similar residues are colored black and yellow boxed, identical residues are colored white and red boxed. Alignment is truncated at the C-terminus of Rab33B. Alignment was done with T-Coffee Expresso [5], Figure was made using ESPript 3.0 [116]. 
Although the overall Atg16L1-bound Rab33B structure is very similar to GppNHp-bound Rab33B (PDB code 1Z06) and GDP bound Rab33B (PDB code 2G77, [103]) significant conformational differences are observed in the switch regions and the site of Atg16L1 interaction. GDP bound Rab33B (PDB code 2G77) shows different switch region conformations due to GDP binding in comparison with the GTP bound Rab33B structure (Fig. 3.1.20.).

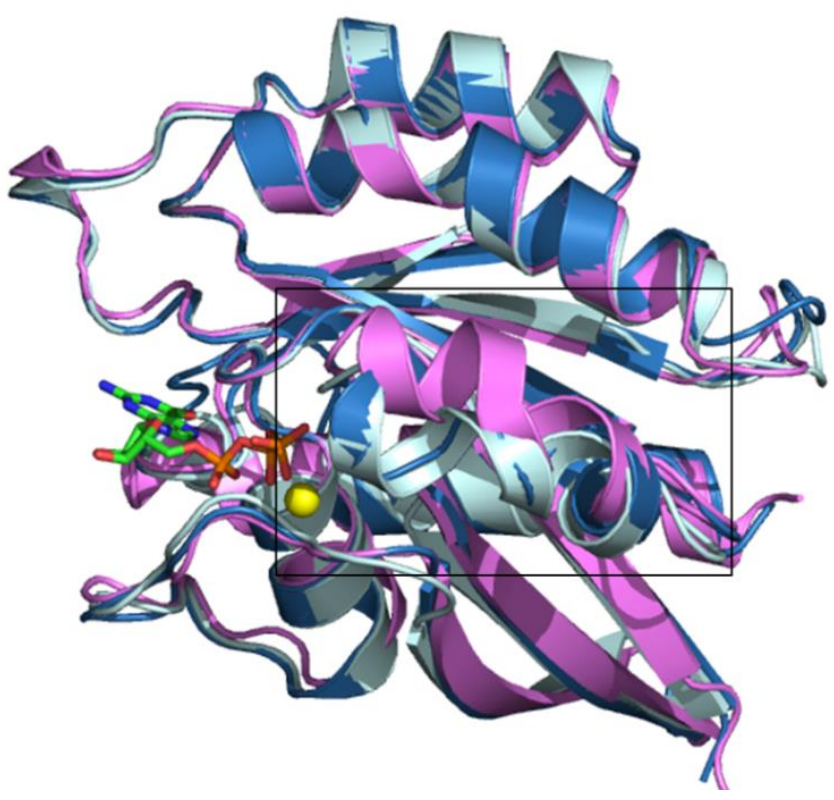

Figure 3.1.20.: Overlay of Rab33B structures in different nucleotide bound states. Cartoon representation of mRab33B in dark turquoise with bound GTP as green stick model and $\mathrm{Mg}^{2+}$ as a yellow sphere superimposed with GppNHp-bound Rab33 (PDB code 1Z06) in blue and GDP-bound Rab33 (PDB code 2G77) in pink. Black box marks the Switch II region.

Although GppNHp-bound Rab33B and Rab33B bound to Atg16L1 are both in the active state a different conformation of the F70 side chain is observed (Fig. 3.1.21.). Interestingly residue F70 was later shown to be essential for Atg16L1 binding both in vivo and in vitro. Two Rab33B molecules interact with the diverging C-termini of an Atg16L1 dimer. Atg16L1 residues 191 to 208 interact with Rab33B, which is consistent with the results of the coexpression experiments to find the minimal interacting coiled coil domain of Atg16L1. Here, truncating the Atg16L1 C-terminal end from 210 to 200 abolished Rab33B binding. 

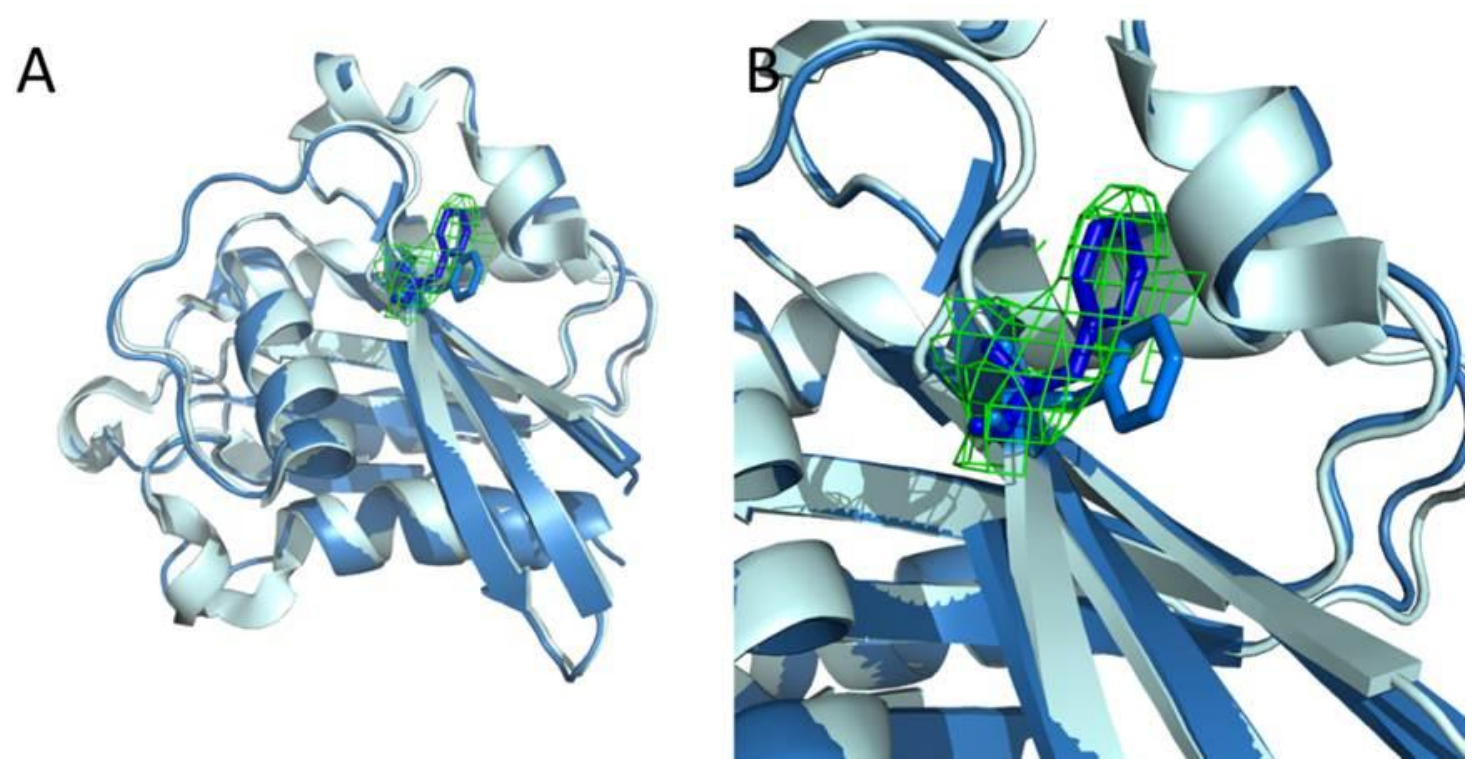

Figure 3.1.21.: Overlay of Rab33B structures in the active state. Cartoon representation of Atg16 bound mRab33B in light blue superimposed with GppNHp-bound Rab33 (PDB code 1Z06) shown in blue. Residue F70 is shown as stick in dark blue for Rab33B and light blue for GppNHp-bound Rab33. Omit map of F70 from Atg16L1 bound Rab33B is countered at $2 \sigma$ in green and clearly demonstrate the different side chain conformation of F70 in both structures.

The geometry of the Atg16L1 coiled coil domain was analyzed using the program TWISTER [130]. The program calculates the local coiled-coil radius in $\AA$ (cc_rad) along the coiled coil axis as a function of residue number. Poly-alanine coordinate files of the three Atg16L1 dimers were used for calculations. The results show a normal coiled-coil radius until residue 189 and from this residue onwards the C-termini are constantly diverging (Fig. 3.1.22).

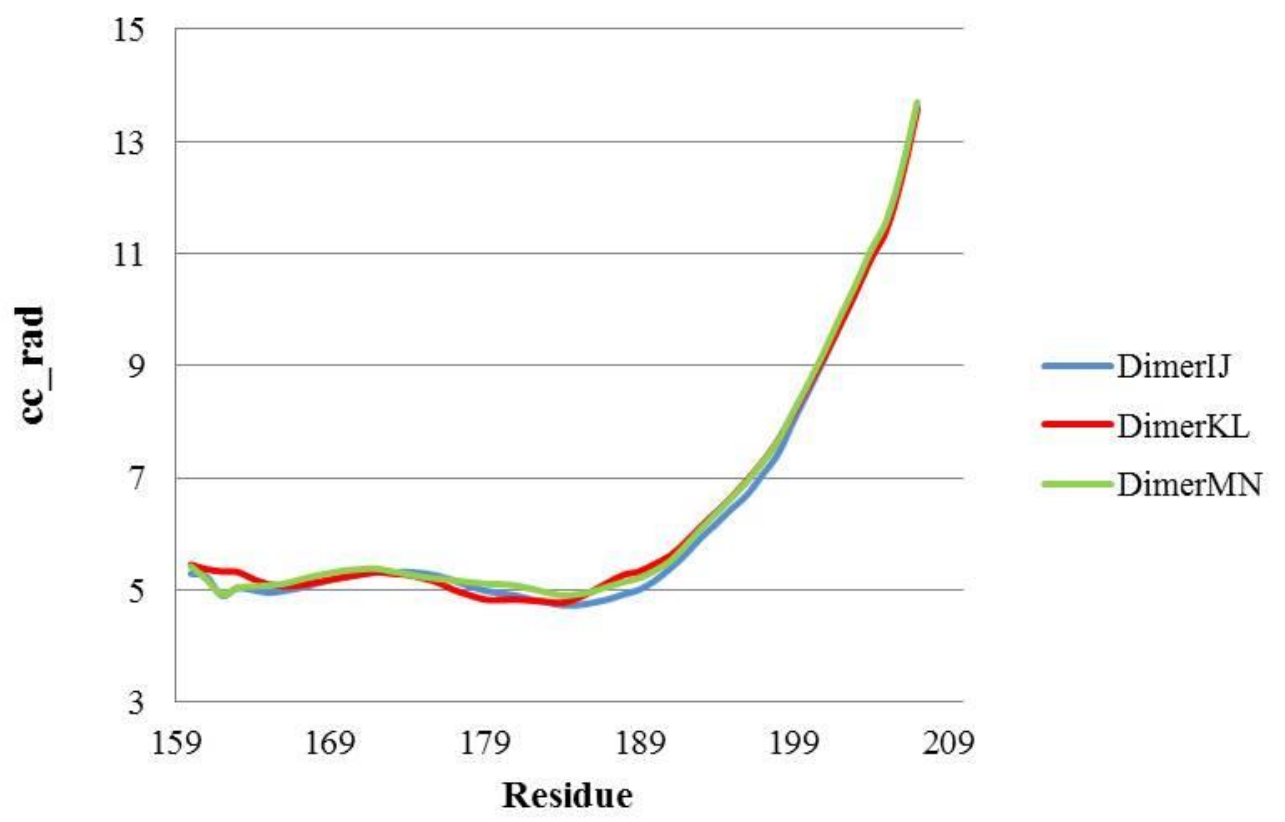


Figure 3.1.22.: Twister analysis of Atg16L1 dimers. The local coiled-coil radius in $\AA$ (cc_rad) along the coiled coil axis is shown as a function of residue number. Analysis shows the divergence of the Ctermini starting at residue 189. Calculations were done with TWISTER [130].

Additionally, an ideal mAtg16L1(153-210) coiled coil dimer was modeled using CCBuilder Version 1.0 [145] and superimposed with the actual Atg16L1 ccd in complex with Rab33B (Fig. 3.1.23). The diverging C-terminus is clearly visible. Analyzing the heptad repeat pattern of Atg16L1 reveals the typical repeating pattern "hxxhcxc" of hydrophobic (h) amino acids at position a and $\mathrm{d}$ and charged (c) amino acids at position e and $\mathrm{g}$ for the first four heptad repeats (Fig. 3.1.24.). The four heptad repeats (residues 187-208) at the C-terminus do not completely obey this pattern with mainly polar and acidic residues at position a (residues 187, 201, 208) and d (residues 183, 204) and hydrophobic residues at position e (residues 191, 198, 205).

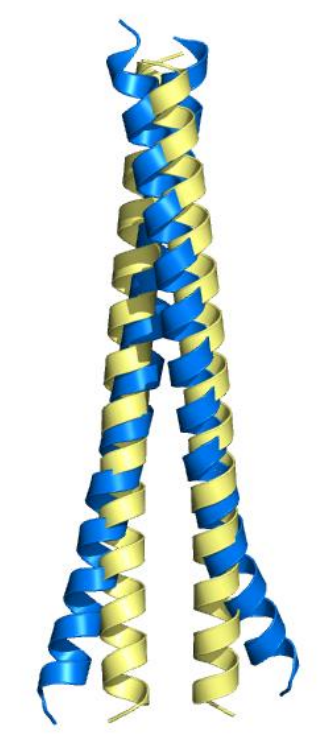

Figure 3.1.23.: Overlay of the Atg16L1 structure and an ideal Atg16L1 model. Cartoon representation of Rab33B bound Atg16L1 dimer IJ in blue superimposed with an ideal Atg16L1 coiled coil dimer model in yellow. Ideal Atg16L1 coiled coil dimer model was made using CCBuilder Version 1.0 [145]. 


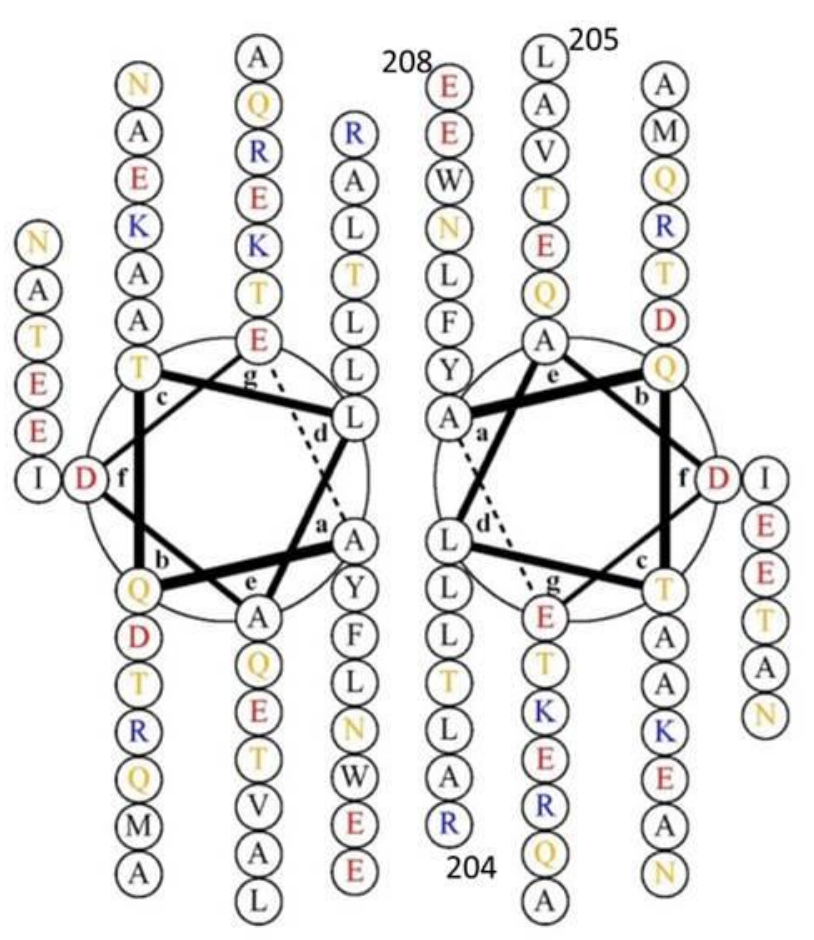

Figure 3.1.24.: Helical wheel projection of the residues in the Atg16L1 dimer IJ. Black: hydrophobic residues, Yellow: polar residues, Red: acidic residues, Blue: basic residues. Numbers indicate residue number. Helical wheel was made using DrawCoil 1.0 [46].

\subsubsection{Cross-linking of the Rab33B-Atg16L1 complex and analysis by mass spectrometry}

Protein cross-linking and analysis by mass spectrometry gives further insights into proteinprotein interactions. Here, the mRab33(30-202)Q92L-mAtg16L1(153-210) complex was cross-linked using the amine-reactive bis(sulfosuccinimidyl) suberate (BS3) at different concentrations. The amount of cross-linking was analyzed by native gel electrophoresis (Fig. 3.1.25.). Without cross-linker only the two bands of Rab33B and Atg16L1 were observed. After the addition of BS3 more bands with a higher molecular weight appeared. The amount of high molecular weight bands increased with the cross-linker concentration. Two bands, marked with black arrows (Fig. 3.1.6.) were chosen and sent for mass spectrometry analysis. This work was done in collaboration with Dr. Momchil Ninov (Department of Neurobiology) and Dr. Chung-Tien Lee (Bioanalytical Mass Spectrometry). 


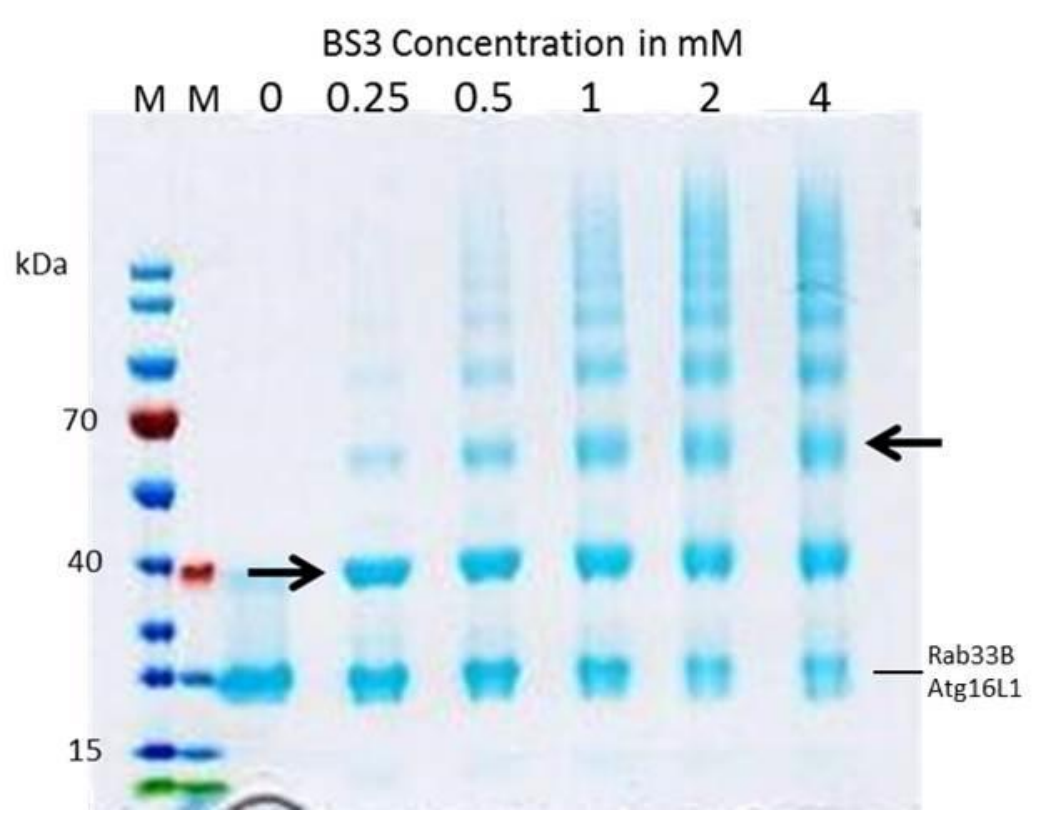

Figure 3.1.25.: Cross-linking of the Rab33B-Atg16L1 complex. Native gel electrophoresis of the Rab33B-Atg16L1 complex (5 mg/ml), cross-linked with 0, 0.25, 0.5, 1, 2 and $4 \mathrm{mM} \mathrm{BS3}$. Arrows mark bands that were analyzed by mass spectrometry. Left arrow flags band1, right arrow band2. M: Marker.

Bands were cut from the gel, digested with trypsin and subjected to LC-MS/MS analysis. Each sample measured in two technical replicates. Raw data were searched against a FASTA database containing the full length sequences of mRab33B and mAtg16L1 using the targetdecoy strategy of pLink. Results are shown with the count of spectrum and the maximum score for each cross-link (Table 3.1.8.). Cross-links were visualized by web-based xiNET [26] (Fig. 3.1.26.). To exclude false positive cross-links the threshold for the maximal score was set to 5.1. The cross-linker used in this study BS3 has a length of $11.4 \AA$ and it preferentially cross-links the zeta nitrogen atom of lysines (NZ). Since lysine sidechains are often conformational flexible and proteins are dynamic in solution a maximum distance of $\sim 24-30$ $\AA$ between $\mathrm{C} \alpha$ atoms of two cross-linked lysines is feasible. Moreover, maximum $\mathrm{C} \alpha-\mathrm{C} \alpha$ distances of $\sim 40 \AA$ were observed [87].

The cross-linked lysine residues were mapped onto the Rab33B, Atg16L1 and Rab33BAtg16L1 structure and $\mathrm{C} \alpha-\mathrm{C} \alpha$ distances were measured using PyMol to visualize and analyze the cross-links (Fig. 3.1.27 - 3.1.29). Rab33B K97 localized in the switch II (89-98) region was the prominent intra molecular cross-linking site (Table 3.1.8., Fig. 3.1.27.). Inter molecular cross-links for mAtg16L1 support that the protein forms a parallel coiled coil dimer (Table 3.1.8., Fig. 3.1.28.). Seven intermolecular cross-links were found between Rab33B and Atg16L1 (Table 3.1.8., Fig. 3.1.29.). The most prominent cross-links occurred between Rab33B K97 and Atg16L1 K179 and K182. Interestingly Atg16L1 K198 that is important for 
complex formation was cross-linked to three Rab33B residues namely K35, K97 and K149. Rab33B residues K46, K165 and K198 that formed intra molecularly cross-links but lay in distance to the Atg16L1 binding site were not cross-linked with Atg16L1.
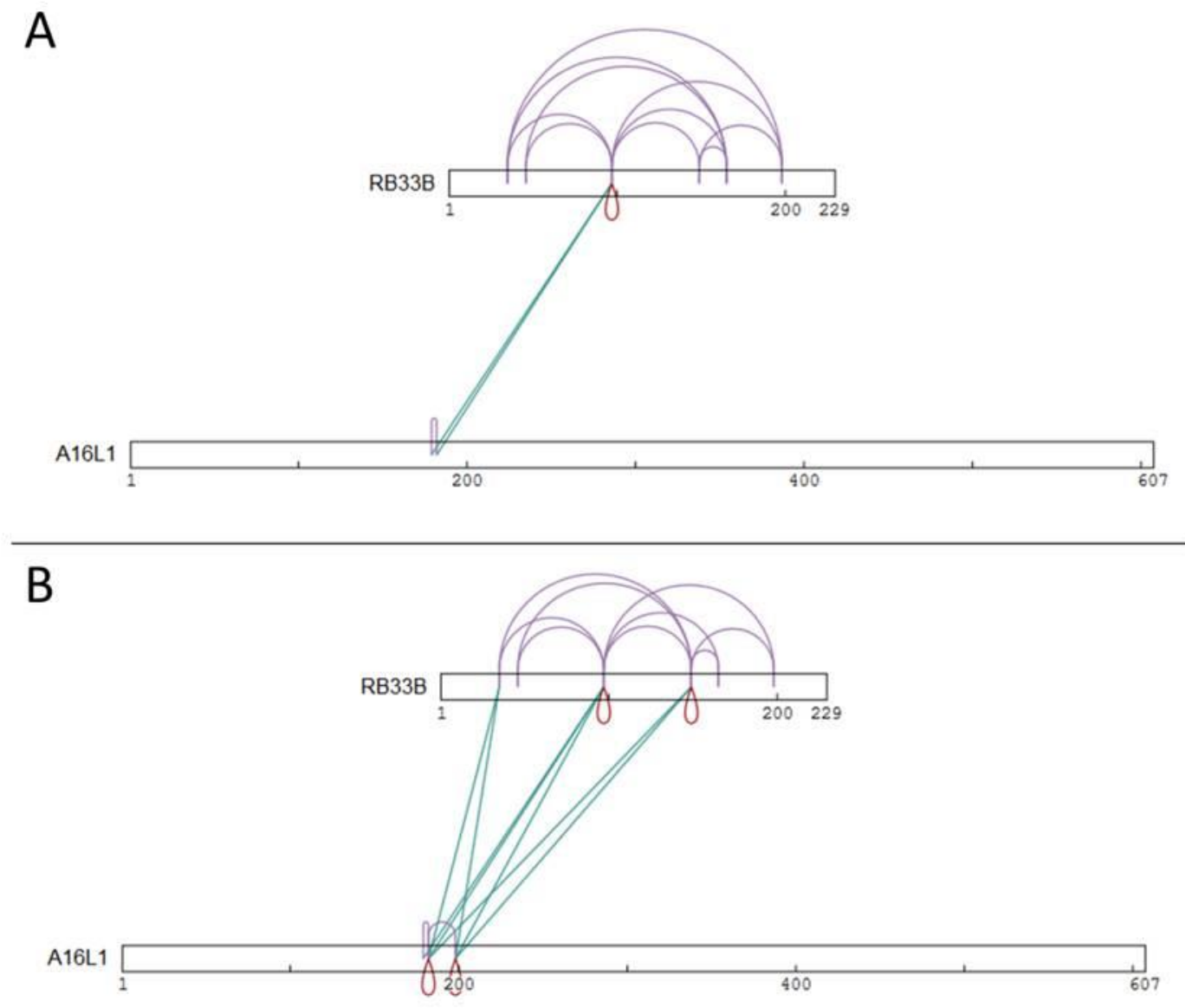

Figure 3.1.26.: Visualization of the mass spectrometry analysis of the cross-linking of the Rab33B-Atg16L1 complex. A: shows analysis of band1, B: shows analysis of band2 from Fig 3.1.6. Samples were run in two technical replicates. Threshold was set to 5.1. Turquoise lines inter crosslinks between Rab33B and Atg16L1. Purple lines inter- or intra molecular cross-links, Red line: Intermolecular self-link. Figures made with xiNET. 
Table 3.1.8.: Data of the mass spectrometry analysis of the cross-linking of the Rab33B-Atg16L1 complex. Samples were run in two technical replicates. Threshold was set to 5.1.

\begin{tabular}{|c|c|c|c|c|c|c|c|}
\hline \multirow[b]{2}{*}{ Protein1 } & \multirow[b]{2}{*}{ Residue1 } & \multirow[b]{2}{*}{ Protein2 } & \multirow[b]{2}{*}{ Residue2 } & \multicolumn{2}{|c|}{ Band1 } & \multicolumn{2}{|c|}{ Band2 } \\
\hline & & & & $\begin{array}{l}\text { Count of } \\
\text { spectrum }\end{array}$ & $\begin{array}{c}\text { Max of } \\
\text { score }\end{array}$ & $\begin{array}{l}\text { Count of } \\
\text { spectrum }\end{array}$ & $\begin{array}{c}\text { Max of } \\
\text { score }\end{array}$ \\
\hline \multirow[t]{7}{*}{ Rab33B } & 97 & Atg16L1 & 182 & 3 & 12.82 & 9 & 22.46 \\
\hline & 97 & & 179 & 3 & 15.52 & 8 & 18.28 \\
\hline & 97 & & 198 & & & 1 & 13.11 \\
\hline & 35 & & 182 & & & 5 & 14.77 \\
\hline & 35 & & 198 & & & 4 & 8.46 \\
\hline & 149 & & 182 & & & 4 & 13.01 \\
\hline & 149 & & 198 & & & 2 & 7.45 \\
\hline \multirow[t]{4}{*}{$\operatorname{Atg16L1}$} & 182 & $\operatorname{Atg16L1}$ & 198 & & & 5 & 20.26 \\
\hline & 179 & & 182 & 2 & 15.7 & 5 & 14.03 \\
\hline & 198 & & 198 & & & 4 & 10.4 \\
\hline & 182 & & 182 & & & 2 & 6.98 \\
\hline \multirow[t]{11}{*}{ Rab33B } & 97 & Rab33B & 35 & 10 & 16.36 & 57 & 21.56 \\
\hline & 97 & & 46 & 6 & 18.34 & 6 & 20.57 \\
\hline & 97 & & 149 & 7 & 9.59 & 15 & 14.03 \\
\hline & 97 & & 198 & 6 & 17.76 & 2 & 13.54 \\
\hline & 97 & & 165 & 8 & 12.86 & 7 & 12.93 \\
\hline & 97 & & 97 & 2 & 5.8 & 23 & 10.63 \\
\hline & 149 & & 46 & & & 2 & 11.03 \\
\hline & 149 & & 165 & 3 & 7.3 & 4 & 7.83 \\
\hline & 149 & & 198 & 2 & 5.23 & 2 & 5.51 \\
\hline & 35 & & 149 & & & 5 & 7.9 \\
\hline & 35 & & 198 & 2 & 5.83 & & \\
\hline
\end{tabular}




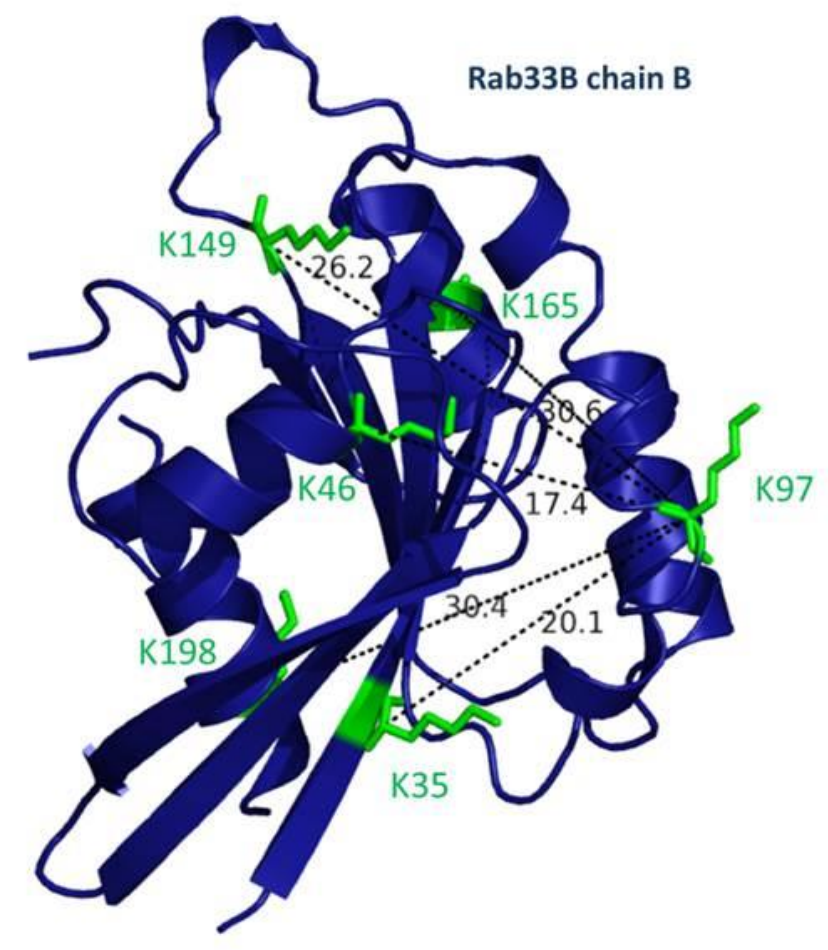

Figure 3.1.27.: Intra molecular cross-links of Rab33B K97. Cartoon representation of Rab33B in dark blue. Lysines are presented as sticks in green. $\mathrm{C} \alpha-\mathrm{C} \alpha$ distances ( $\AA$ ) between K97 and intra molecular cross-links to other lysine residues are shown as dashed lines. Figure was made with Pymol.

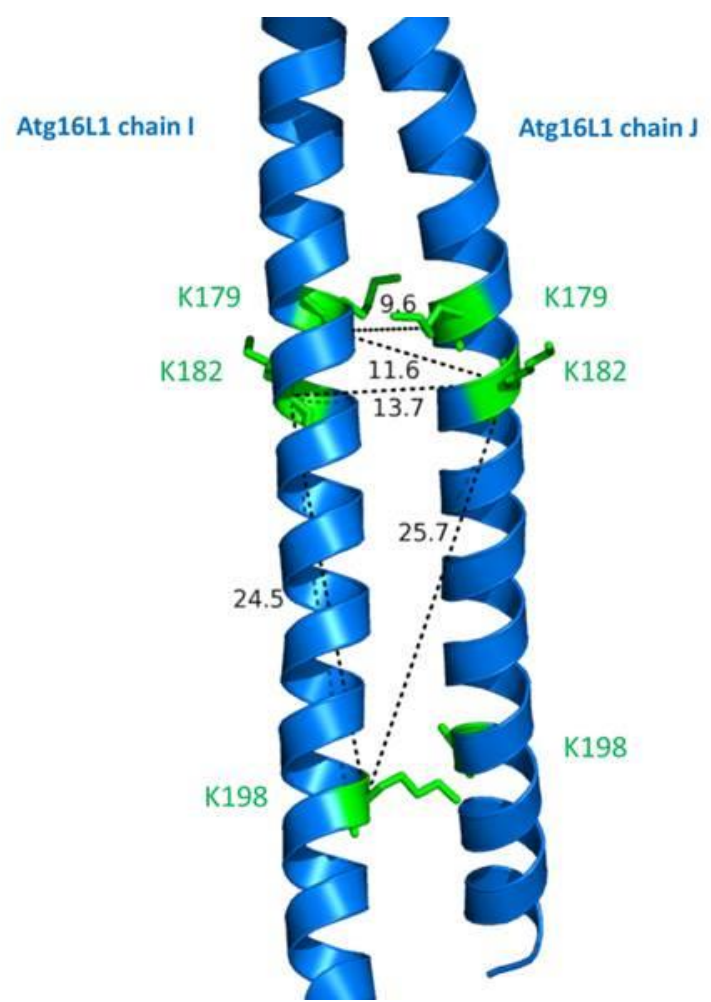

Figure 3.1.28.: Intra molecular cross-links of Atg16L1. Cartoon representation of Atg16L1 in blue. Lysines are presented as sticks in green. $\mathrm{C} \alpha-\mathrm{C} \alpha$ distances $(\AA)$ between intra molecular cross-links are shown as dashed lines. Figure was made with Pymol. 


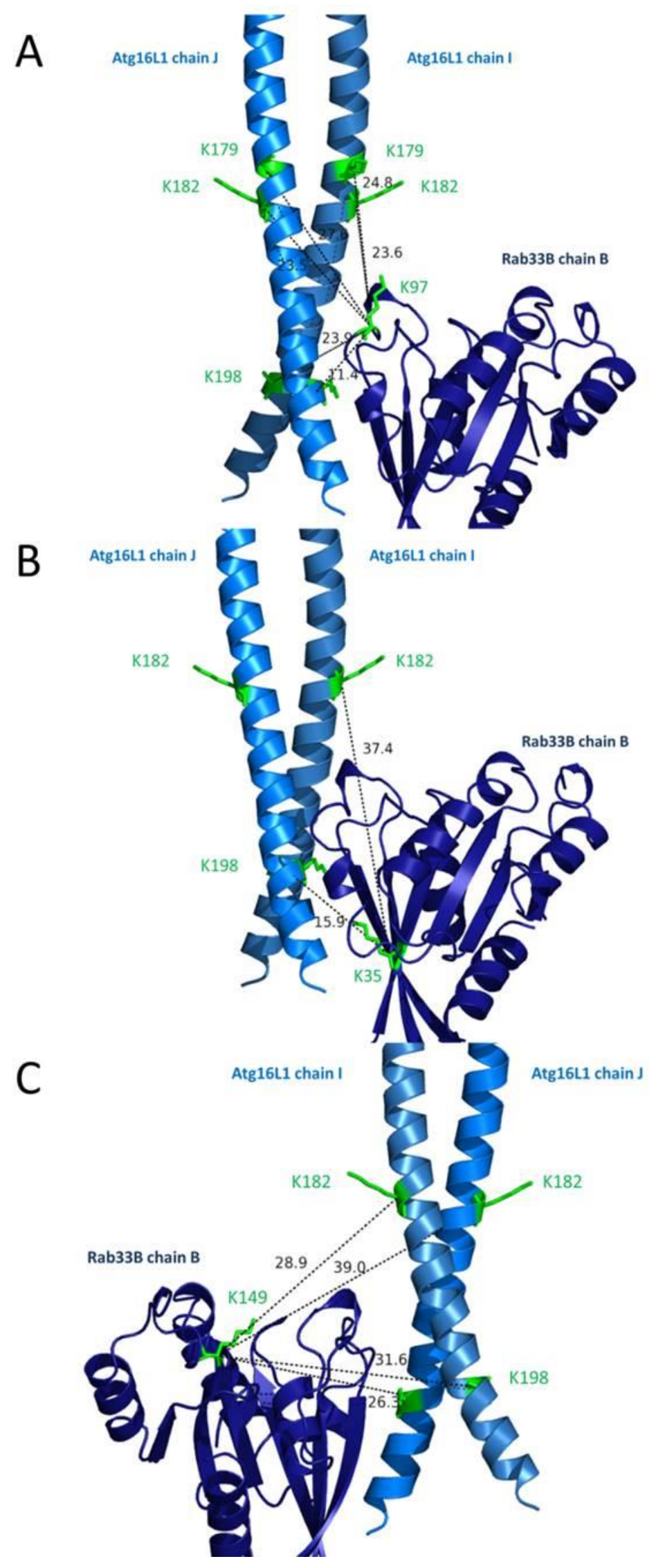

Figure 3.1.29.: Cross-links between Rab33B and Atg16L1. Cartoon representation of Rab33B in dark blue and Atg16L1 in blue. Lysines are presented as sticks in green. C $\alpha$-C $\alpha$ distances $(\AA)$ between intra molecular cross links are shown as dashed lines. Figure was made with Pymol. 


\subsubsection{Analyzing complex formation of Rab33B and Atg16L1 mutants in vitro}

Based on the structure of the Rab33B(30-202)Q92L-Atg16L1(153-210) complex and literature search for residues that were mutated in other Rab proteins [148, 151], mutants were designed to interrupt complex formation of Rab33B and Atg16L1. Three mutations were selected for each protein at positions that are likely to contribute to complex formation (Fig. 3.1.30.). For Rab33B aromatic residues F70 and W87 were mutated. Both residues are strongly conserved in Rab GTPases (Fig. 3.1.19.). Residue F70, that is part of the switch I region, was chosen because it undergoes a conformational change upon binding to Atg16L1 (Fig. 3.1.21.). Two mutations were selected for Rab33B residue F70. The F70A mutation removes the aromatic side chain and thus gives insights on the importance of the phenyl-side chain for complex formation. The F70E mutation changes this amino acid position from neutral to a negative charge.

Tryptophan is the largest amino acid with aromatic and nonpolar characteristics; mutation W87A changes this position to a small hydrophobic amino acid. For Atg16L1 mutation K198A changes the flexible amino acid with a positive charged $\varepsilon$-amino group to a small hydrophobic amino acid. For A202W a steric clash would be expected that inhibits complex formation. With mutation N206K the neutral amino acid is changed to positively charged residue. Only residue N206 is conserved between mammalian and yeast Atg16 (Fig. 3.1.11.)

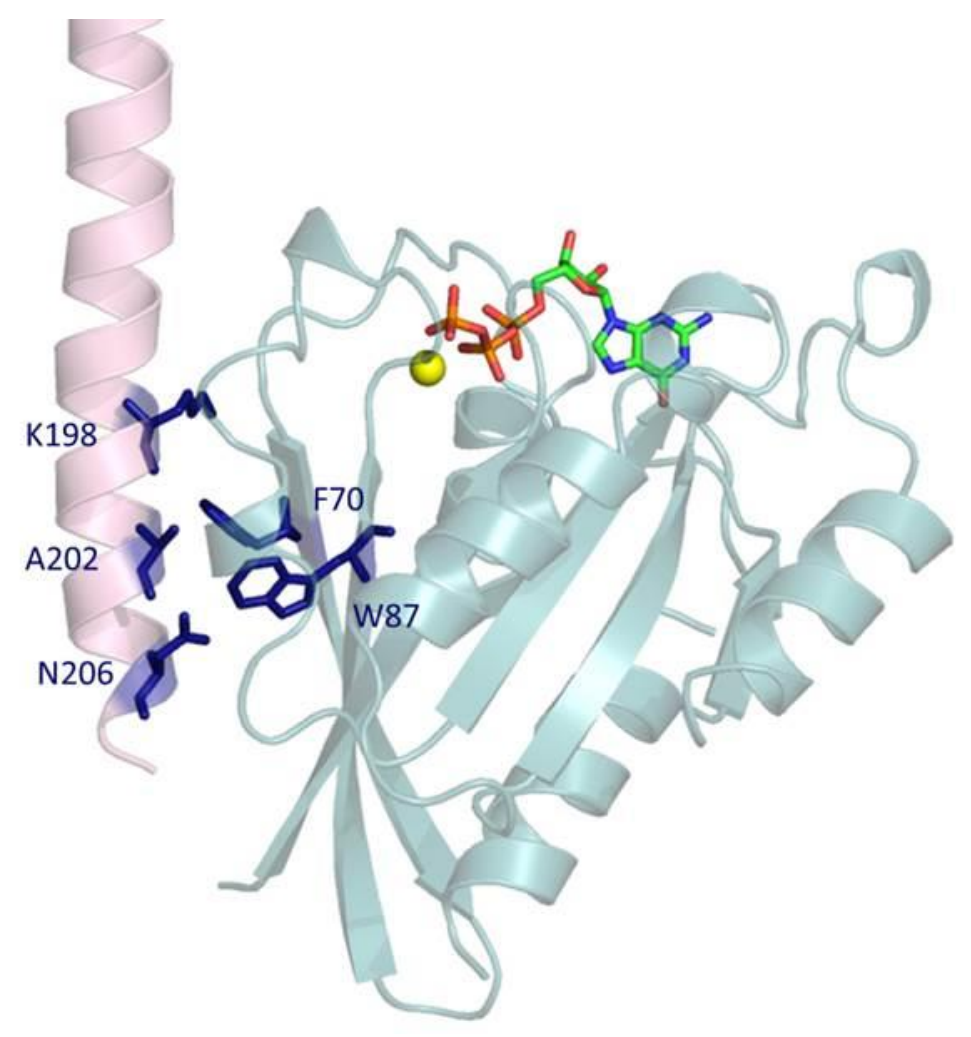


Figure 3.1.30.: Rab33B-Atg16L1 mutations. Transparent cartoon representation of in light turquoise bound ligands GTP as green carbon stick model and magnesium as yellow sphere and Atg16L1 in light pink. Residues selected for mutagenesis F70, W87 (Rab33B) and Atg16L1 residues K198, A202 and N206 are shown in stick representation in dark blue.

Rab33B(30-202)Q92L-Atg16L1(153-210) mutants were also co-expressed in pETDuet1 vector with a His-tagged Rab33B and untagged Atg16 as described previously. A pull-down assay with Ni-sepharose beads was performed to examine complex formation. Schägger gel electrophoresis was performed for all pull down steps in order to verify expression of both proteins and to track proteins. As an example the pull down of Rab33B(30-202)Q92LAtg16L1(153-210)K198A is shown (Figure 3.1.31.). Both proteins are expressed as seen in the supernatant fraction. After binding to Ni-sepharose beads the Atg16L1 band is visible in the flow through and the amount of Atg16L1 decreases with every washing step when unbound protein is washed from the beads. Finally, Rab33B elutes alone indicating that no complex was formed between Rab33B(30-202)Q92L and Atg16L1(153-210)K198A.

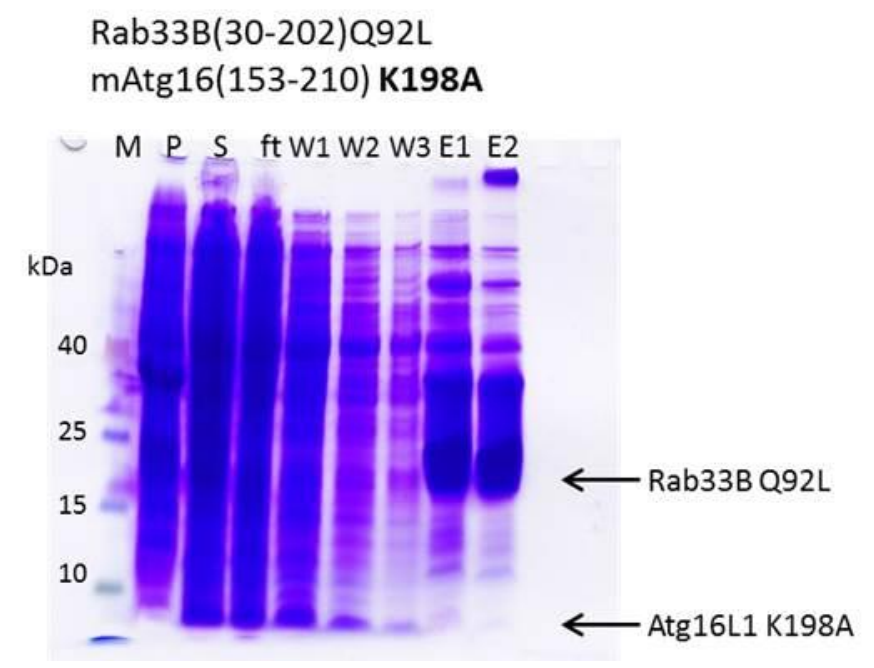

Figure 3.1.31.: Ni-sepharose pulldown of Rab33B(30-202)Q92L and Atg16L1(153-210) K198A. Schägger gel electrophoresis of samples from all purification steps. Arrows indicate bands for Rab33B(30-202)Q92L and mAtg16L1(153-210) K198A. M: Marker, P: Pellet, S: Supernatant, ft: flow through, W1-3: Wash 1-3, E1-2: Elution 1-2.

Ni-Sepharose elution fractions of all mutants were separated by Schägger gel electrophoresis and subjected to western blotting. One membrane was probed with anti-His Tag antibody to detect Rab33B and the other was probed with anti-Atg16L1 antibody (Fig. 3.1.32.). Complex formation of Atg16L1(153-210)WT was also tested with Rab33B(30-202)WT, GTP-bound Rab33B(30-202)Q92L and GDP-bound Rab33B(30-202)T47N. Immunodetection revealed that Rab33B(30-202)WT and Q92L bound Atg16L1(153-210) in similar amounts, whereas the Rab33B(30-202)T47N did not bind Atg16L1 in vitro. This observation confirms that 
Atg16L1 is an effector of Rab33B. Additionally, it can be concluded that Rab33B(30-202) WT is predominantly present in the GTP-bound form. Importantly, all single point mutations that were introduced in either Rab33B or Atg16L1 disrupted complex formation in vitro.

A anti-His-Tag Antibody

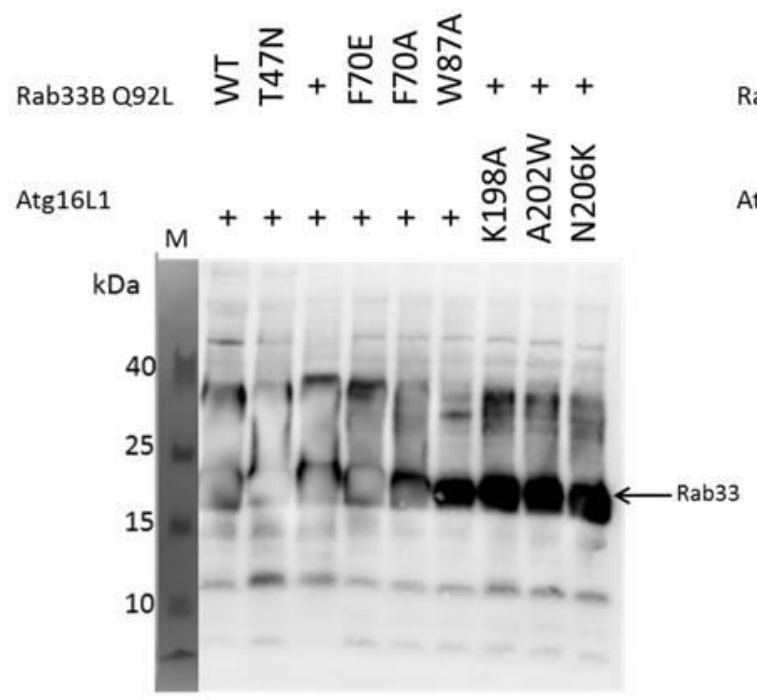

B

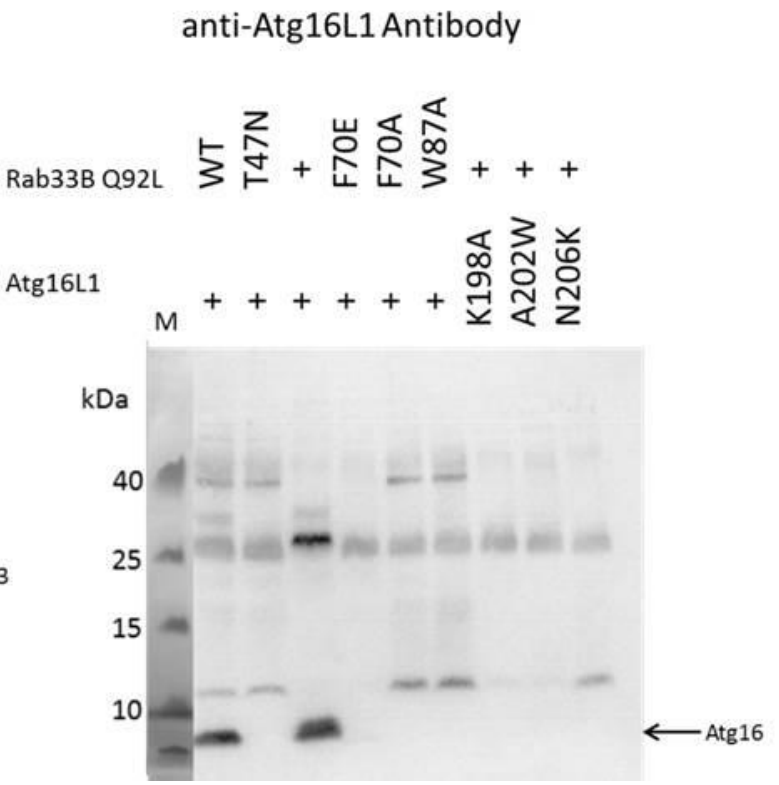

Figure 3.1.32.: Ni-Sepharose pulldown of Rab33B(30-202) and $\operatorname{Atg} 16 \operatorname{L1}(153-210)$. Ni-Sepharose elution fractions were used for Schägger gel electrophoresis and blotted on two nitrocellulose membranes. Membranes were probed with A: Penta His HRP conjugate antibody or B: rabbit antiAtg16L primary antibody and goat anti-rabbit IgG (HRP labeled) secondary antibody. M: Marker. Rab33B mutations WT and T47N did not contain the Q92L mutation, whereas F70E/A and W87A were mutated additional to Q92L.

\subsubsection{In vivo characterization of the Rab33B-Atg16L1 complex}

To further investigate complex formation of Rab33B and Atg16L1 and examine the influence of the Rab33B-Atg16L1 complex on autophagy in vivo studies were conducted with Dr. Beyenech Binnotti (Department for Neurobiology).

\subsubsection{Expression of full length Rab33B and Atg16L1 in HEK293 cells}

For in vivo expression full length mRab33B Q92L and WT were cloned into mammalian expression vector pcDNA3.1/nV5. F70A/E and W87A mutations were additional introduced for mRab33B Q92L and mRab33B WT was altered to the GDP-trapped mutant T47N. For full length mAtg16L1 in mammalian expression vector pcDNA6.1/C-EmGFP mutations K198A, A202W and N206K were prepared. 
pcDNA3.1/nV5 Rab33B Q92L was co-transfected with pcDNA6.1/C-EmGFP mAtg16L1 WT, K198A, A202W and N206K for overexpression in HEK293 cells. To test the effect of the Rab33B mutants pcDNA6.1/C-EmGFP mAtg16L1 WT was co-transfected with pcDNA3.1/nV5 Rab33B WT, T47N or Q92L F70A/E and Q92L W87A. Overexpression levels were tested by western blotting, membranes were probed with anti-GFP or anti-V5 primary antibody and IR dye 800 secondary antibody. All Atg16L1 variants were overexpressed at equivalent levels and the same was observed for all Rab33B variants (Fig. 3.1.33.).

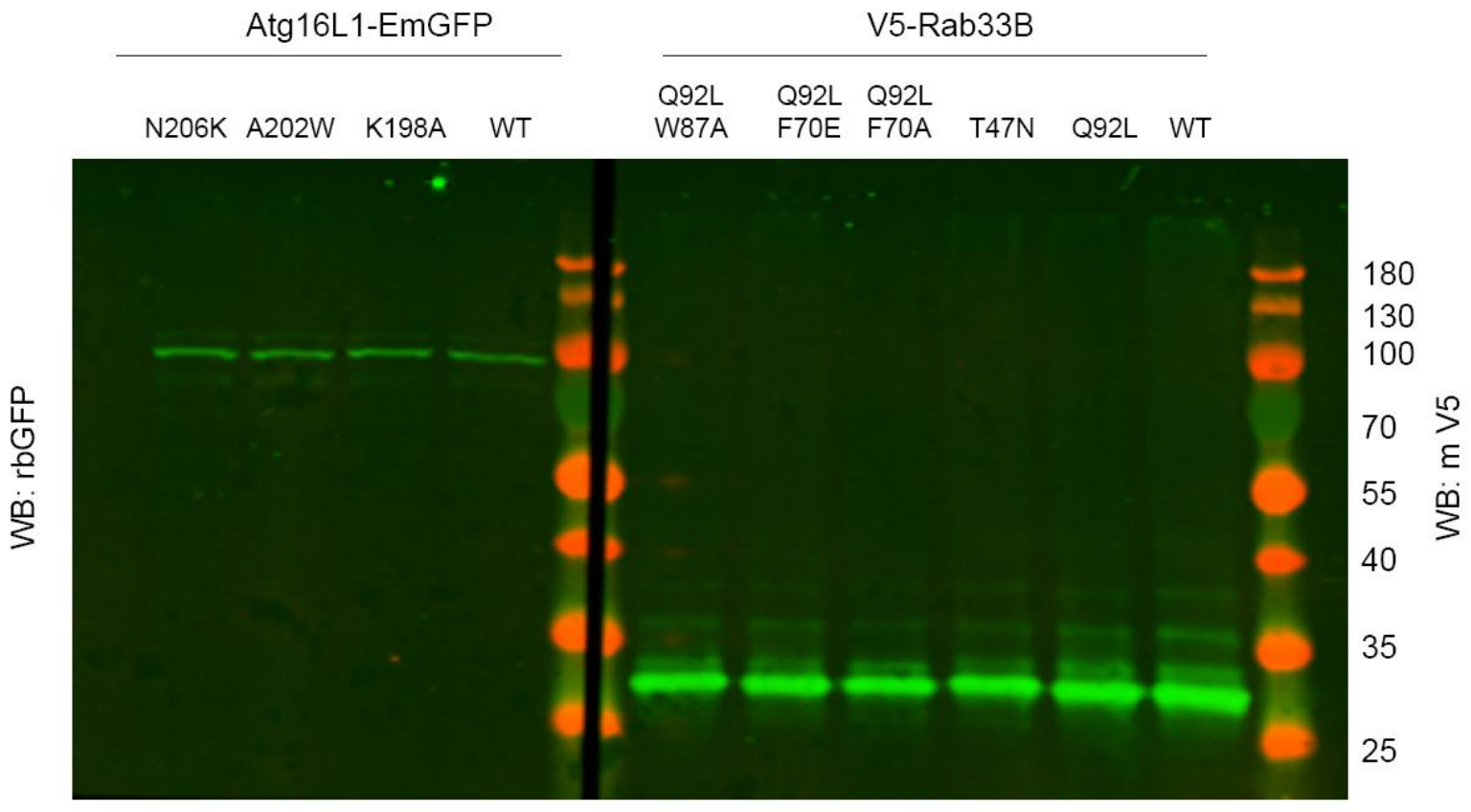

Figure 3.1.33.: Western blot analysis to characterize the overexpression of Atg16L1-EmGFP and V5-Rab33B. Overexpression was done in HEK cells. Western blot membranes were probed Membranes were probed with rabbit anti-GFP or mouse anti-V5 primary antibody and IR dye 800 (green) secondary antibody for Odyssey fluorescence detection. Marker in red.

\subsubsection{Immunoprecipitation and pull down assays}

Co-immunoprecipitation experiments were conducted to evaluate complex formation of Rab33B and Atg16L1 in vivo. HEK293 cells overexpressing full length mRab33B and mAtg16L1 constructs were lysed and incubated with a GFP-antibody. The GFP-antibody was immobilized with protein A dynabeads. GFP-antibody-beads capture GFP-tagged Atg16L1 and all proteins that form a direct or indirect complex with Atg16L1. After GFP-pull down proteins were eluted and submitted to SDS gel electrophoresis followed by western blotting. Success of the immunocoprecipitation was verified by Atg16L1 staining with a GFP antibody. 
Rab33B bound to Atg16L1 was detected with a V5-antibody. To test for lipidation membranes were also probed with specific antibodies for endogenous LC3B (Fig. 3.1.34.).

Analysis of the western blot revealed that Atg16L1 WT, A202W and N206K were efficiently and equally extracted by the anti GFP-antibody. Only Atg16K1 K198A was extracted in lower amounts but repetition of the experiment showed similar amounts of all Atg16L1 variants (data not shown). In the presence of Atg16L1, both WT Rab33B WT and Q92L coprecipitated in similar high amounts. When Atg16L1 WT was co-expressed with the GDPbound Rab33B T47N a significantly lower amount of Rab33B T47N co-precipitated. In contrast, no complex formation was observed in pull down experiments with the Rab33B(30202) T47N mutant and WT mAtg16L1(153-210) in vitro. Complex formation of Rab33B Q92L with Atg16L1 was disrupted for all mutants expect for Atg16L1 WT and Rab33B Q92L F70E which showed a significantly reduced level of complex formation compared to Rab33B Q92L with Atg16L1 WT.

The lipidation state of LC3 was analyzed with a LC3B specific antibody. The antibody detects a band of approximately $17 \mathrm{kDa}$ for the PE-modified LC3-II form and a $19 \mathrm{kDa}$ band for unlipidated LC3-I. Both LC3 forms were only detected in the input demonstrating that no direct binding to Atg16L1 occurred as expected. Interestingly, the level of LC3-II was increased when the constantly active Rab33B Q92L was overexpressed as observed before [54]. The increased LC3-II levels are independent of complex formation with Atg16L1. 


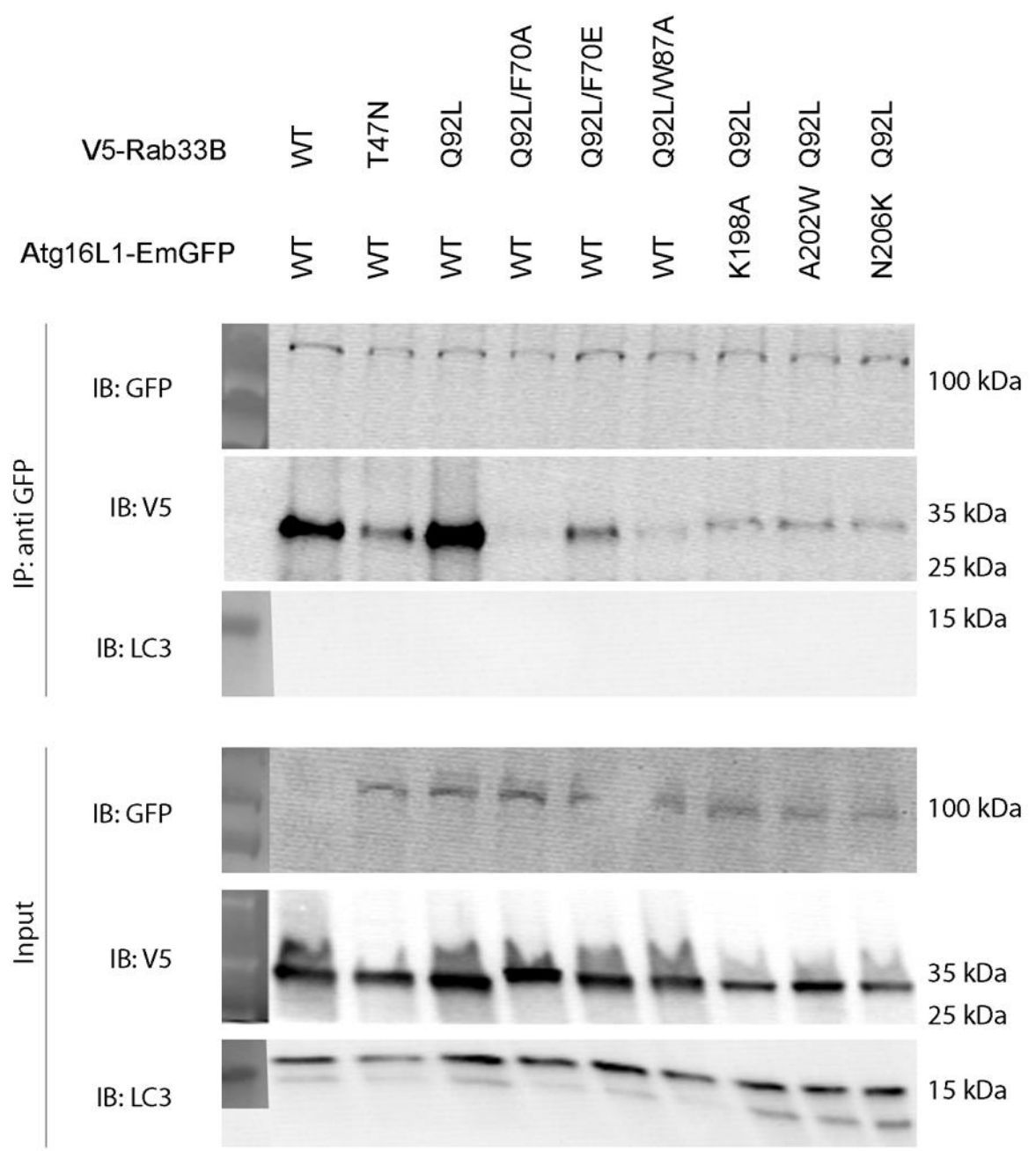

Figure 3.1.34.: GFP co-immunoprecipitation of Atg16L1-EmGFP and V5-Rab33B variants. Overexpression was done in HEK293 cells. Western blots were probed with either anti-GFP, anti-V5 or anti-LC3B antibodies.

Additionally to the GFP pulldown a V5 pulldown was performed to validate these findings (Fig. 3.1.35.). The experiment was conducted as before, but an anti V5-antibody was used for immobilization. Co-immunoprecipitation using the V5-antibody demonstrated the same complex formation pattern for the Rab33B and AtgL1 constructs. The highest amount of Atg16L1 WT pull down was observed for Rab33B WT and its Q92L mutant. The GDPtrapped Rab33B T47N mutant did not co-precipitated Atg16L1 WT. A low amount of coimmunoprecipitation was observed for Rab33B Q92L F70E with Atg16L1 WT and for Rab33B WT with Atg16L1 K198A or N206K. However, no complex formation was observed for the other Rab33B or Atg16L1 mutants. 


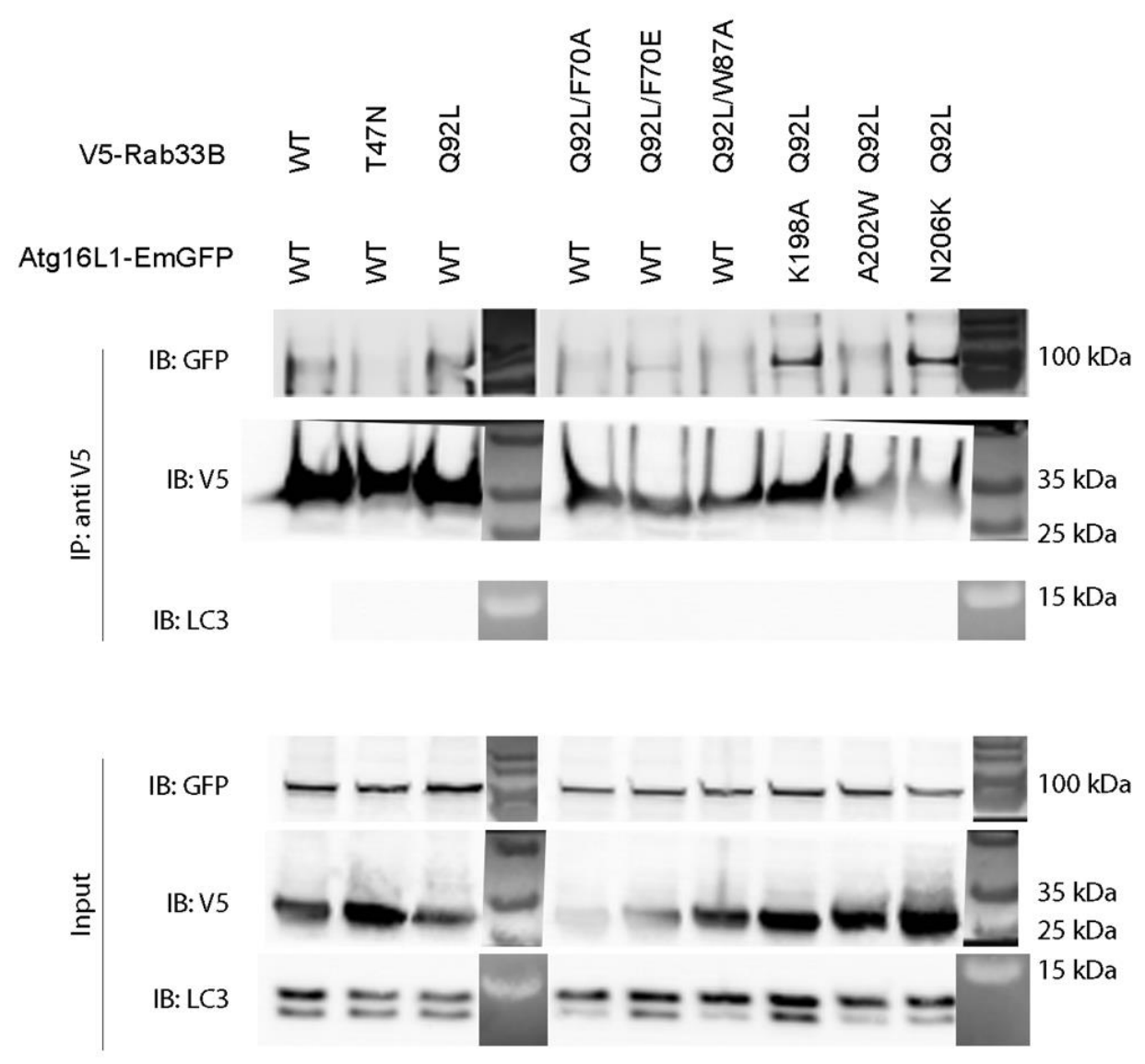

Figure 3.1.35.: V5 co-immunoprecipitation of Atg16L1-EmGFP and V5-Rab33B variants. Overexpression was done in HEK293 cells. Western blots were probed with either anti-GFP, anti-V5 or anti-LC3B antibodies.

\subsubsection{MAP1LC3 Lipidation assay}

To further verify the finding of increased LC3-II levels when the constantly active Rab33B Q92L mutant was overexpressed a MAP1LC3 lipidation assay was performed. Microtubuleassociated proteins 1A/1B light chain 3B (MAP1LC3), hereafter named LC3, was detected with a LC3B specific antibody. The antibody detects a band of approximately $17 \mathrm{kDa}$ for the PE-modified LC3-II form and a $19 \mathrm{kDa}$ band for unlipidated LC3-I. Tubulin was detected for quantification of protein levels. Cells were maintained under nutrient conditions or treated with $20 \mathrm{mM} \mathrm{NH}_{4} \mathrm{Cl}$ to block the autophagic flux [92]. Results are in agreement with the observations made in co-immunoprecipitation experiments. Under nutrient conditions LC3-II levels were enhanced for Rab33B Q92L with Atg16L1 WT compared to Rab33B WT or T47N with Atg16L1 WT. Additionally increased LC3-II levels were observed for Rab33B Q92L F70E with Atg16L1 WT and Rab33B Q92L with Atg16L1 mutants. Results show that the effect of Rab33B Q92L on LC3 levels in nutrient rich conditions is independent of complex formation with Atg16L1 like observed before. 


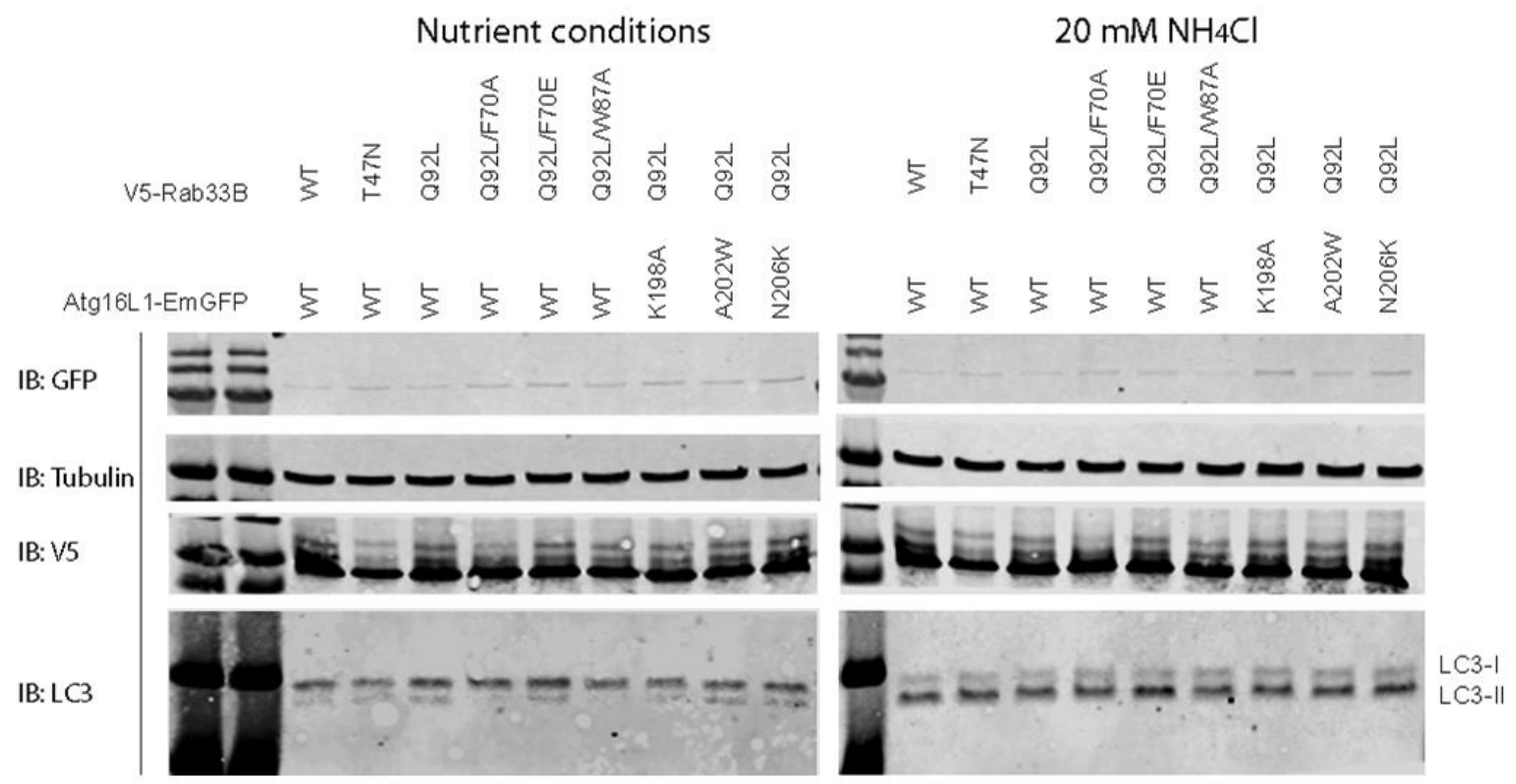

Figure 3.1.36.: MAP1LC3 Lipidation assay. Overexpression was done in HEK293 cells. Cells were maintained under nutrient conditions or $20 \mathrm{mM} \mathrm{NH}_{4} \mathrm{Cl}$. Western blots were probed with either antiGFP, anti-V5, anti-Tubulin or anti-LC3B antibodies.

\subsubsection{Fluorescence microscopy}

In order to study the intracellular distribution and function of the Rab33B-Atg16L1 complex fluorescence microscopy was performed in Cos-7 cells, a fibroblast-like cell line derived from monkey kidney tissue, which was co-transfected with the Rab33B and Atg16L1 variants used for co-immunoprecipitation. In cells co-transfected with Atg16L1 and either Rab33B Q92L or WT punctate structures positive for both proteins were observed in the cytoplasm, demonstrating co-localization (Fig. 3.1.37. A). Expression of GTP-bound Rab33B Q92L with Atg16L1 lead to the observation of enlarged punctuate structures (Fig. 3.1.37. B), whereas the expression of GDP-bound Rab33B T47N with Atg16L1 clearly reduced the amount of punctuate structures compared to Rab33B WT. Furthermore Rab33B T47N is located mostly to the perinuclear area (Fig 3.1.37. C). A dramatic loss of punctuate structures was observed upon expression of the Rab33B Q92L F70A/F70E/W87A mutants with Atg16 L1 or Rab33B Q92L with Atg16L1 K198A, A202W or N206K mutants, respectively (Fig. 3.1.38. D-F and Fig. 3.1.39. G-I). Only few punctuate Atg16L1 positive structures were observed in some of the cells but do not localize with Rab33B. No difference between cells that lack the Rab33BAtg16L1 complex (Fig. 3.1.38. E, F and Fig. 3.1.39. G) and cells with a decreased amount of Rab33B-Atg16L1 complex (Fig. 3.1.38. D and Fig. 3.1.39. H-I) was noticeable. 
A
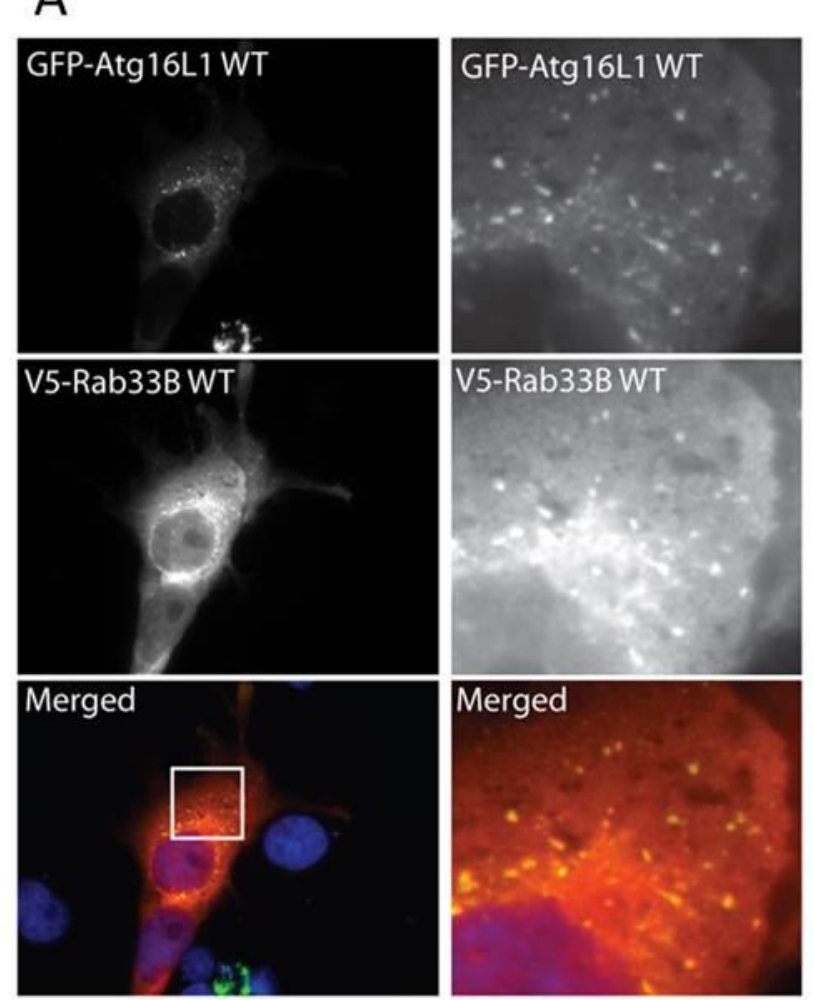

B

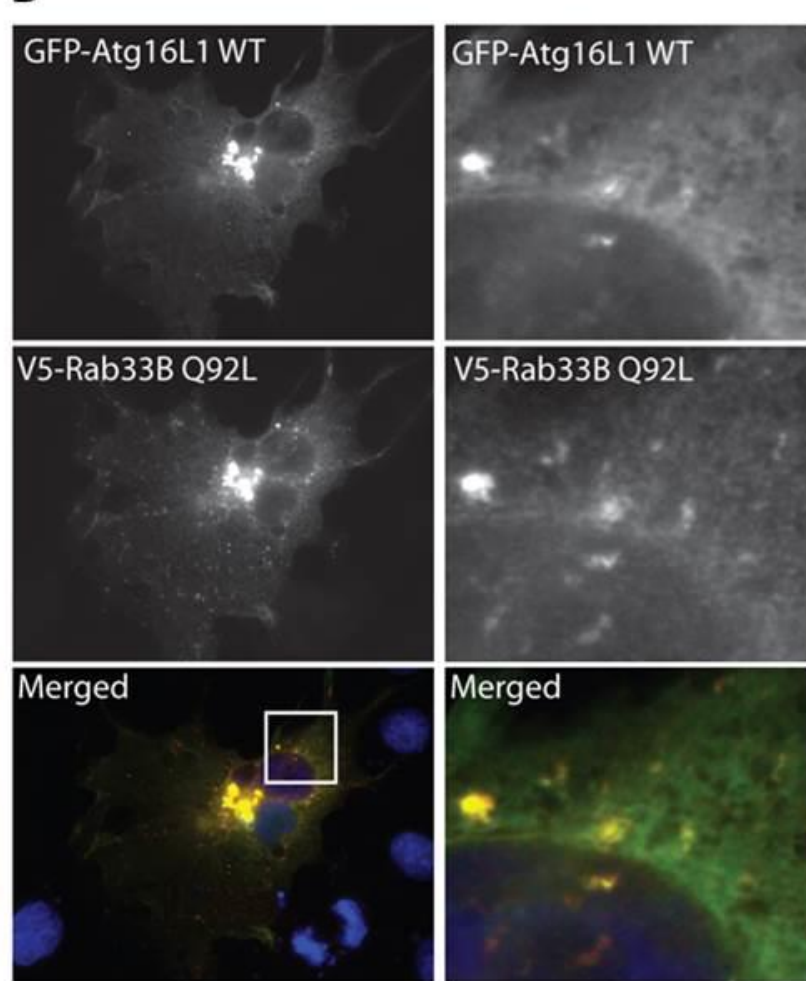

C

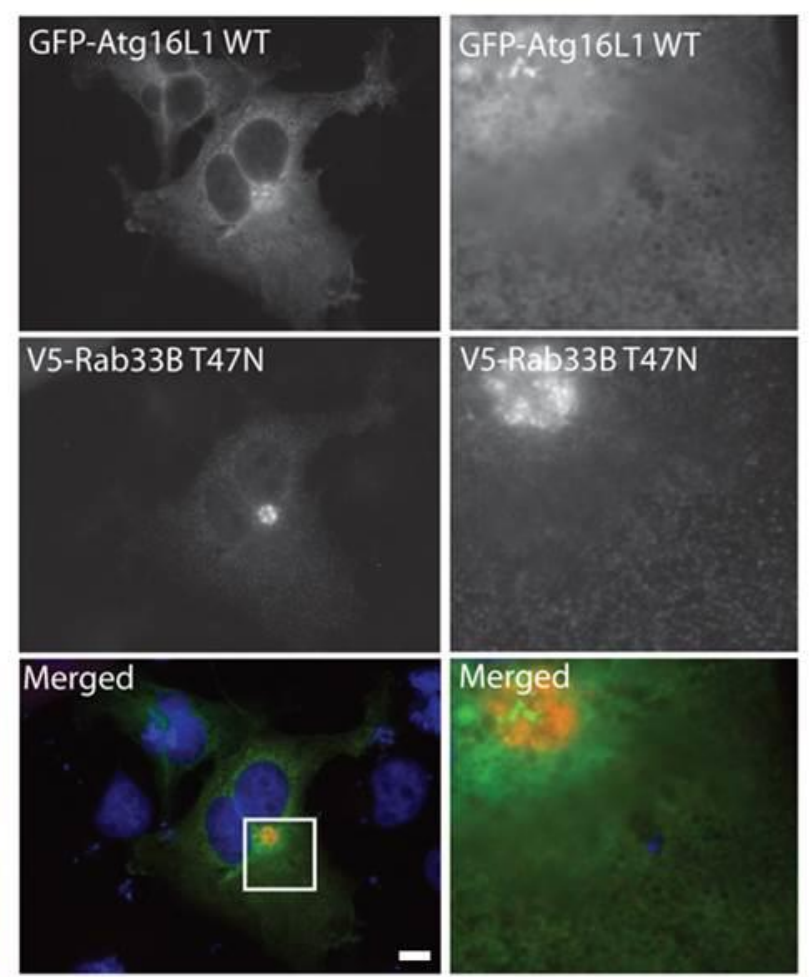

Figure 3.1.37.: Intracellular distribution of EmGFP-Atg16L1 and V5-Rab33B WT/ Q92L or T47N. EmGFP-Atg16L1 and V5-Rab33B variants transiently expressed in Cos-7 cells. Scale bar, $10 \mu \mathrm{m}$. 

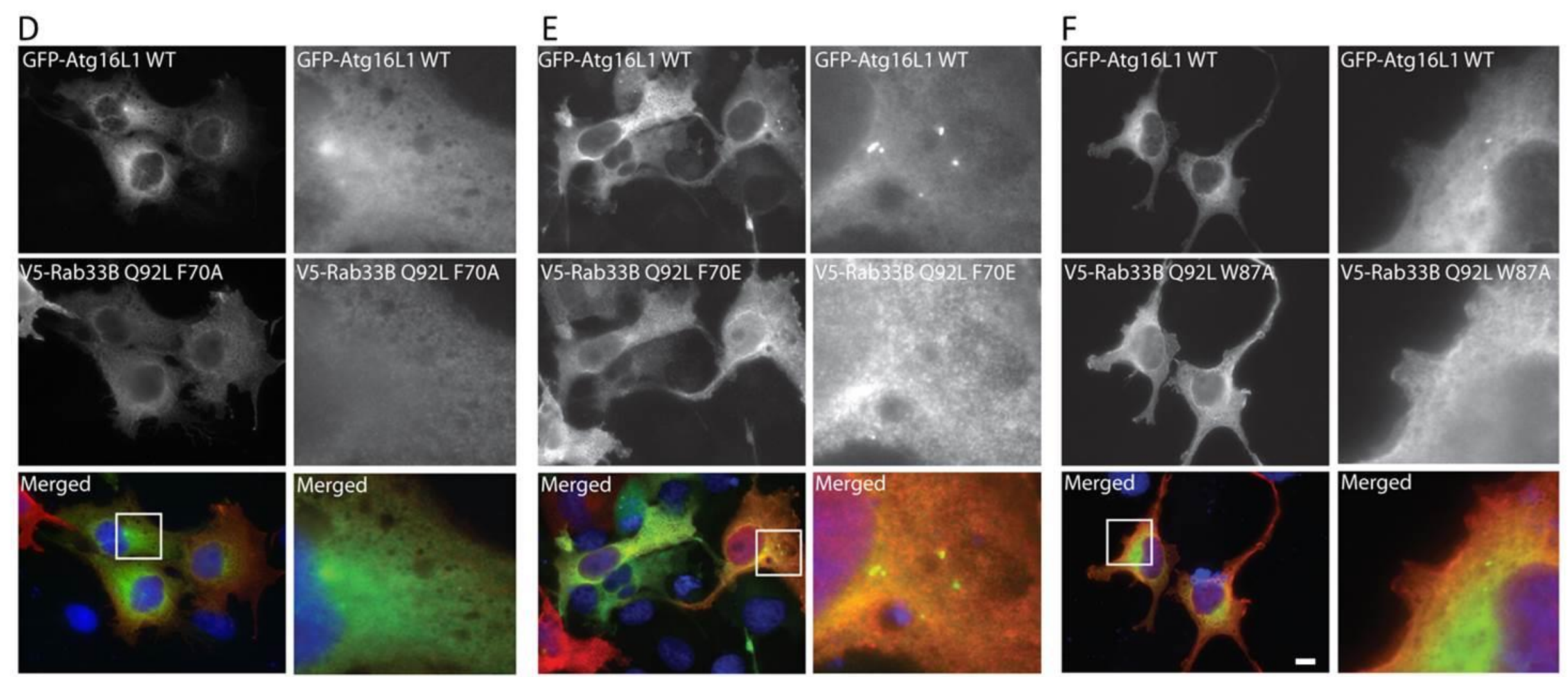

Figure 3.1.38.: Intracellular distribution of EmGFP-Atg16L1 and V5-Rab33B Q92L F70A/ F70E or W87A. EmGFP-Atg16L1 and V5Rab33B variants transiently expressed in Cos-7 cells. Scale bar, $10 \mu \mathrm{m}$. 
G
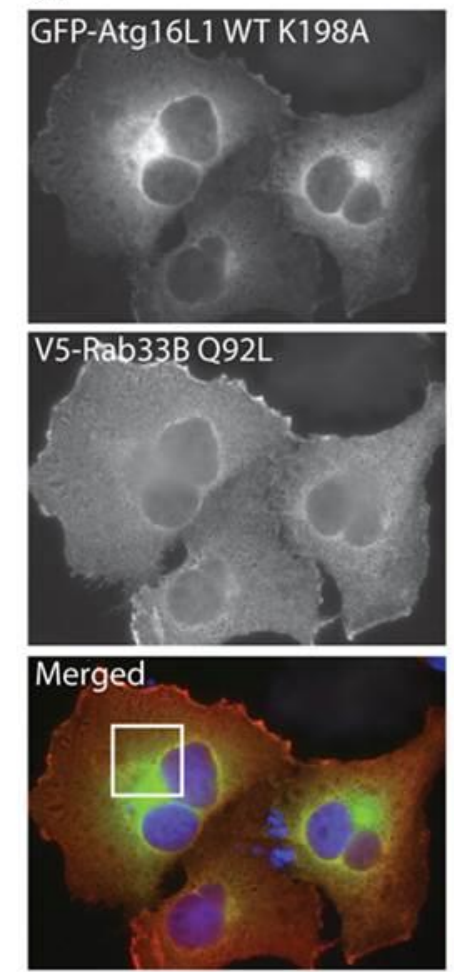

$\mathrm{H}$
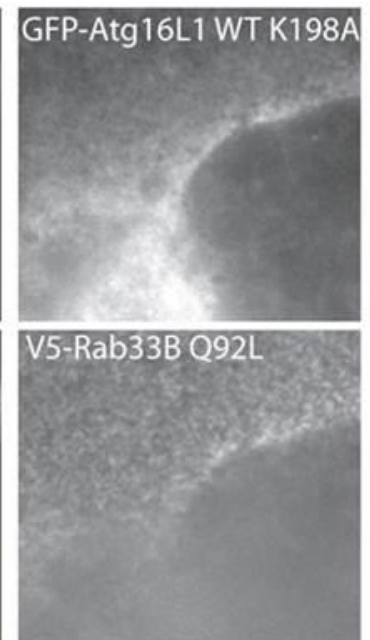

Merged

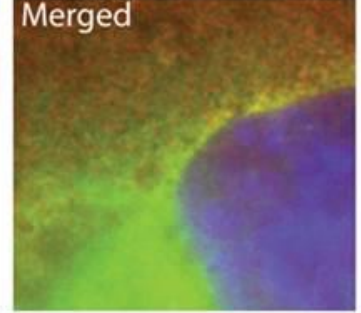

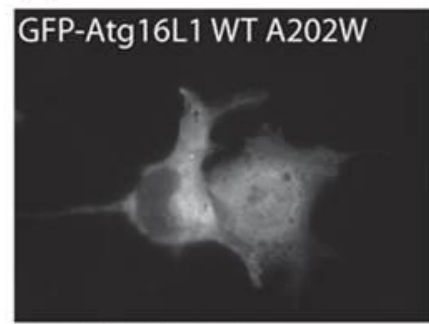
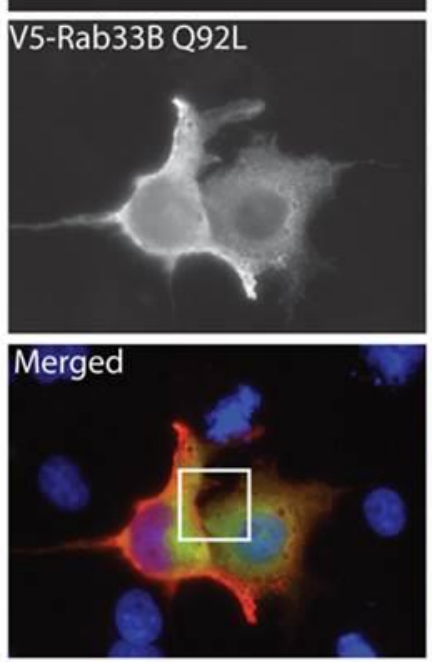
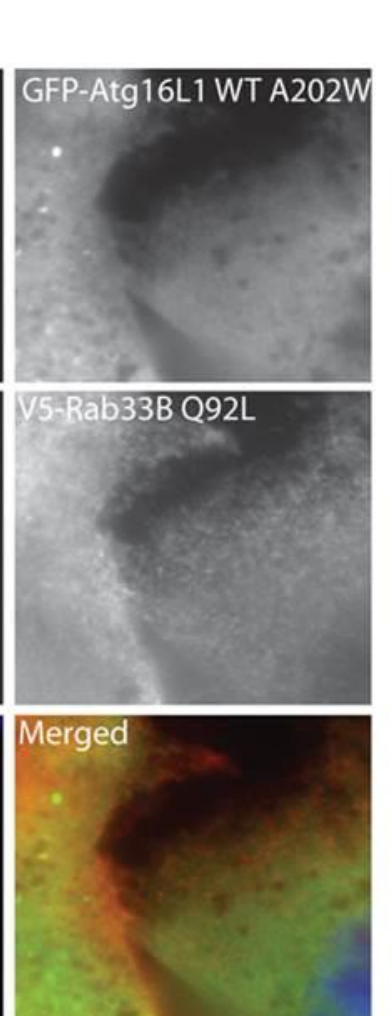
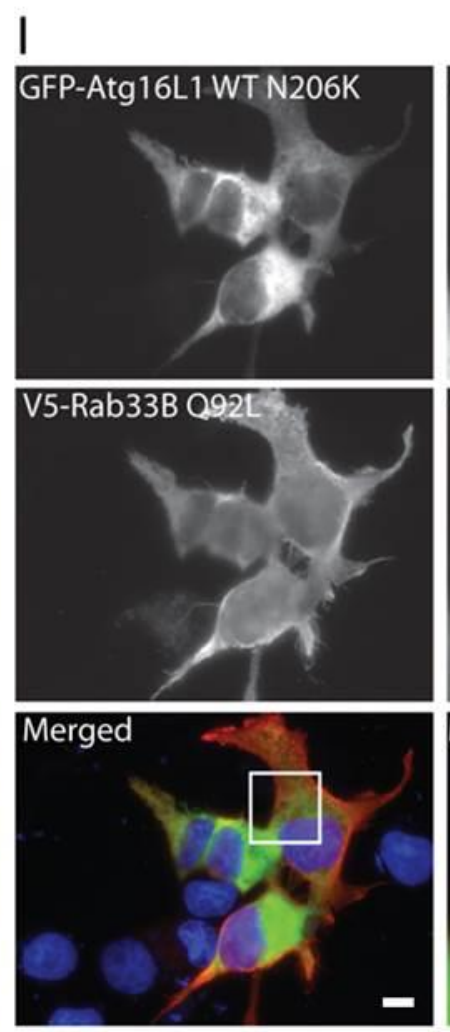

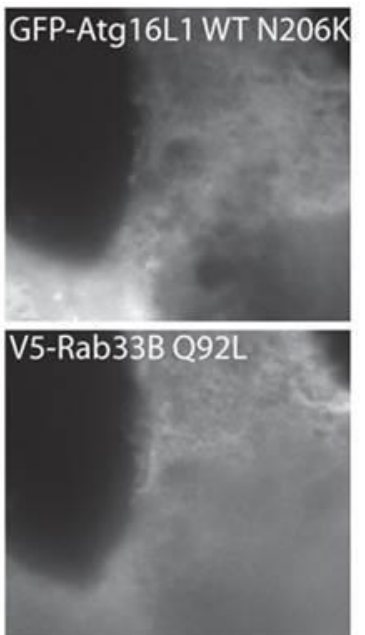

Merged

Figure 3.1.39.: Intracellular distribution of EmGFP-Atg16L1 K198A/ A202W or N206K and V5-Rab33B Q92L. EmGFP-Atg16L1 variants and V5-Rab33B Q92L transiently expressed in Cos-7 cells. Scale bar, $10 \mu \mathrm{m}$. 


\subsection{Characterization of the KIAtg21-AgAtg16 complex}

The Atg21-Atg16 complex coordinates the lipidation of Atg8 by organizing the ubiquitin-like conjugation system at the expanding isolation membrane. Atg16 coiled coil domain residues E102 and D101 are important for Atg21 binding [58]. Further insights into the interaction between Atg21 and Atg16 will help to understand its molecular function.

\subsubsection{Expression and purification of KlAtg21, AgAtg16 and KIAtg21-AgAtg16 complexes}

For expression and purification of the Atg21-Atg16 complex full length Atg21 from yeast Kluyveromyces lactis was chosen. Dr. Andreea Scacioc showed that Saccharomyces cerevisiae Atg21 is insoluble when expressed by E. coli. [119]. K. lactis Atg21 was previously purified in our group [19]. S. cerevisiae and K. lactis Atg21 are conserved and share $40.2 \%$ identity (SIM Expasy). The coiled coil domain of Ashbya gossypii Atg16 interacts with KlAtg21 and was used for further studies because AgAtg21 could not be expressed (K. Kühnel, personal communication). Both KlAtg21 and AgAtg16 were individually expressed in E. coli from a pET28a vector. For purification of the complex expression pellets of KlAtg21 and AgAtg16 were mixed in a ratio of 2:1. Each protein was purified either individually or together for complex isolation with a two-step protocol. The first step was HisTrap chromatography followed by a polishing step with size exclusion chromatography using a HiLoad 16/60 Superdex 200 column (Fig. 3.2.1.). Purification of the complex was conducted by Dr. Karin Kühnel. 
A

\section{B}

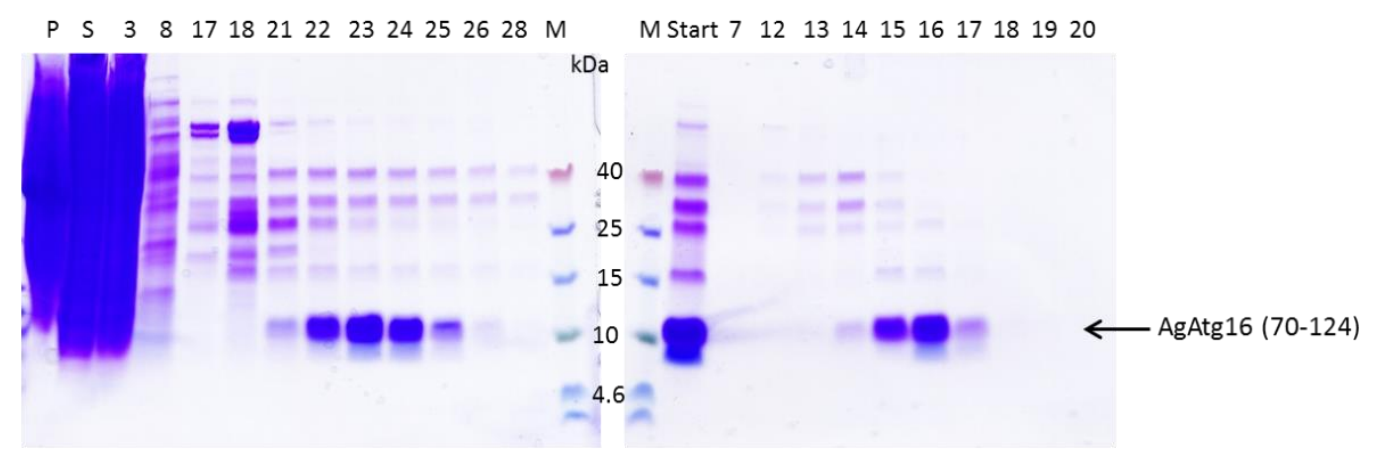

C

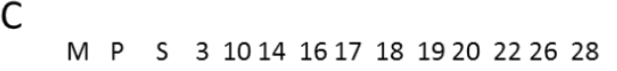

M start $_{7} 91011121314151617182123$

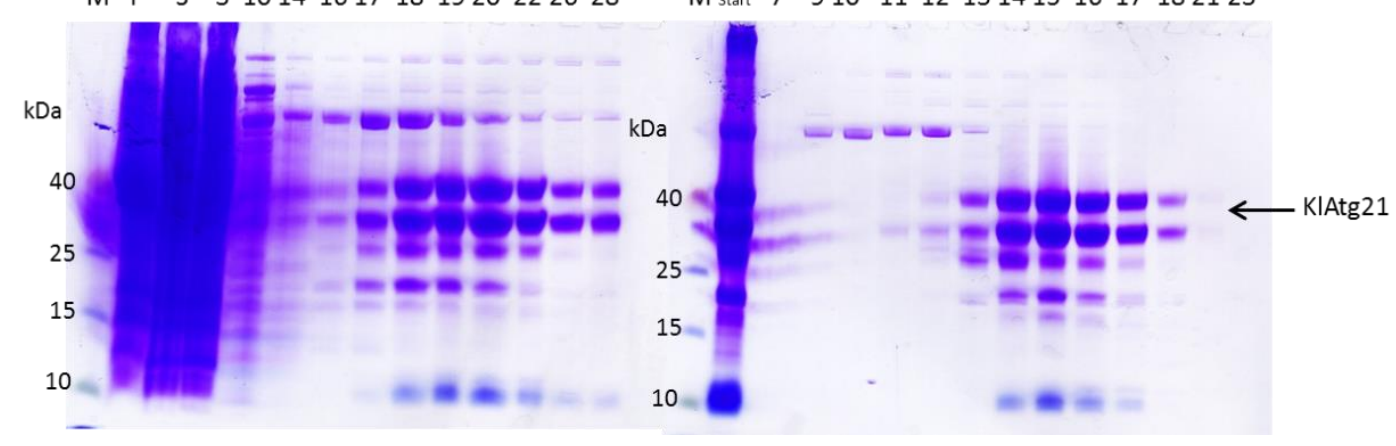

Figure 3.2.1.: Purification of KlAtg21 and AgAtg16 (70-124). Schägger gel analysis of purification steps. A, B: Purification of AgAtg16(70-124). A: HisTrap, B: size exclusion chromatography Superdex 200. C, D: Purification of KlAtg21. C: HisTrap, D: size exclusion chromatography Superdex 200. Of notice, KlAtg21 eluted as double band due to degradation of the protein. M: Marker, Start: Input for SEC. Numbers corresponds to fractions.

\subsubsection{Biochemical characterization of the KIAtg21-AgAtg16 complex}

\subsubsection{Analysis of complex formation by analytical gel filtration}

To minimize the K1Atg21-AgAtg16 complex for crystallization the minimal binding domain of AgAtg16 was searched for. Construct design and initial experiments were conducted by Dr. Karin Kühnel. These preliminary results needed further confirmation. To identify the minimal binding domain of AgAtg16 for complex formation the interaction of full length K1Atg21 with different AgAtg16 constructs was analyzed by analytical gel filtration. Here, $100 \mathrm{nmol}$ KlAtg21 was mixed with $50 \mathrm{nmol}$ AgAtg16, incubated for $30 \mathrm{~min}$ on ice and loaded on a Superdex 200 10/300 GL analytical gel filtration column with $30 \mathrm{mM}$ HEPES pH 7.5, 150 $\mathrm{mM} \mathrm{NaCl}, 1 \mathrm{mM}$ TCEP gel filtration buffer. The chromatogram was compared to individual runs of KlAtg21 and AgAtg16. A peak shift indicates complex formation. Complex formation was observed between full-length KlAtg21 and AgAtg16 (40-124) (Fig. 3.2.2.) and AgAtg16 (70-124) (Fig. 3.2.3.). For KlAtg21-AgAtg16 (40-124) almost all protein formed a complex, 
whereas for the KlAtg21-AgAtg16(70-124) run two peaks representing the complex and unbound protein were observed. Thus, KlAtg21 and AgAtg16(40-124) seemed to form a more stable complex. Only a small peak shift was observed for KlAtg21 with C-terminal truncated AgAtg16 (47-103) (Fig. 3.2.4.) and AgAtg16 (47-103) eluted between fractions 26-32 indicating a weak interaction.

KIAtg21 fl

AgAtg16 (40-124)
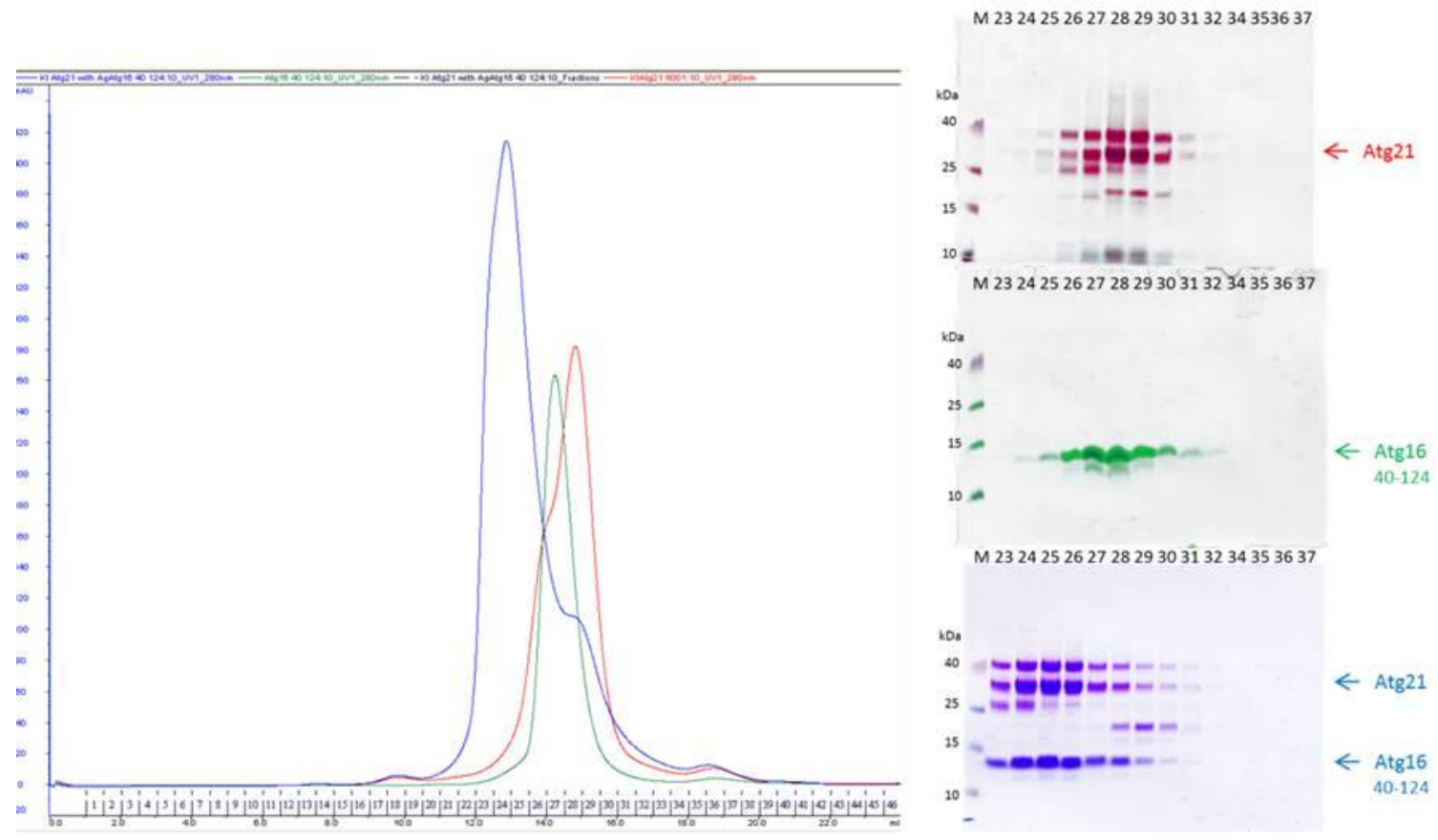

Figure 3.2.2.: Analytic gel filtration of KlAtg21 and AgAtg16 (40-124). Chromatograms of size exclusion chromatography using a Superdex 200 10/300 GL column. Red curve: KlAtg21, green curve: AgAtg 16 (40-124), blue curve: KlAtg21 and AgAtg16 (40-124). Schägger gel electrophoresis of elution fractions are shown on the right side. M=Marker, Numbers refer to elution fractions. Bands were colored according to chromatogram color. Arrows indicate KlAtg21 or AgAtg16 (40-124) respectively. 


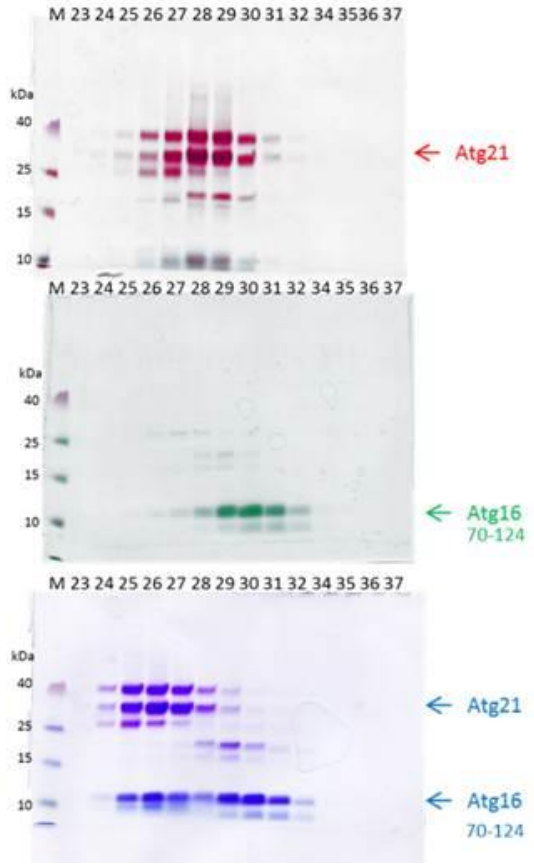

Figure 3.2.3.: Analytic gel filtration of KlAtg21 and AgAtg16 (70-124). Chromatograms of size exclusion chromatography using a Superdex 200 10/300 GL column. Red curve: KlAtg21, green curve: AgAtg16 (70-124), blue curve: KlAtg21 and AgAtg16 (70-124). Schägger gel electrophoresis of elution fractions are shown on the right side. M=Marker, Numbers refer to elution fractions. Bands were colored according to chromatogram color. Arrows indicate KlAtg21 or AgAtg16 (70-124) respectively.

KIAtg21 $\mathrm{fl}$

AgAtg16 (47-103)

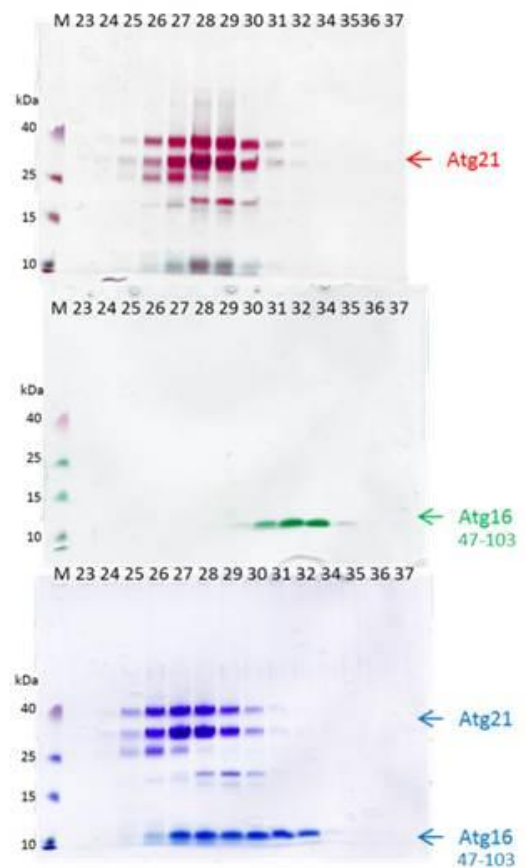


Figure 3.2.4.: Analytic gel filtration of KlAtg21 and AgAtg16 (47-103). Chromatograms of size exclusion chromatography using a Superdex 200 10/300 GL column. Red curve: KlAtg21, green curve: AgAtg16 (47-103), blue curve: KlAtg21 and AgAtg16 (47-103). Schägger gel electrophoresis of elution fractions are shown on the right side. Bands were colored according to chromatogram color. Arrows indicate KlAtg21 or AgAtg16 (47-103) respectively.

\subsubsection{Analysis of the KIAtg21-AgAtg16 complex stoichiometry by SEC-MALLS measurements}

The stoichiometry of the KlAtg21-AgAtg16 (70-124) complex was investigated by SECMALLS measurements in collaboration with Johannes Arens and Dr. Achim Dickmanns (Department for Molecular Structural Biology, University of Göttingen). After separation on a Superdex 200 10/300 GL column samples were run through a MALLS detector. MALLS measurements allow the molecular weight determination of a protein independent of its shape and can therefore be used for the determination of molecular weights of non-globular proteins, for example coiled-coil proteins. Measurements were done for the KlAtg21AgAtg16 complex, KlAtg21 and AgAtg16 individually to analyze the stoichiometry of the complex.

Chromatograms of the size exclusion prior to MALLS measurements showed that the KlAtg21-AgAtg16 (70-124) complex was formed (Fig. 3.2.5.) SEC-MALLS measurements (Fig. 3.2.6. and Table 3.2.1.) of KlAtg21 gave a molecular weight of $43 \mathrm{kDa}$, which is consistent with the calculated molecular weight of $45.7 \mathrm{kDa}$ including the His Tag. For AgAtg16 (70-124) $15 \mathrm{kDa}$ were measured and $15.4 \mathrm{kDa}$ were calculated for an Atg16 dimer including the His Tag. Results also confirm that AgAtg16 is dimeric. Measurements of the molecular weight of the KlAtg21-AgAtg16 (70-124) complex gave a molecular weight of $67 \mathrm{kDa}$. The measured value corresponds to one KlAtg21 molecule in the complex. Because the Atg16 coiled coil domain is most likely to form a dimer it is likely that one KlAtg21 molecule forms a complex with one AgAtg16 dimer, giving a theoretical molecular weight of $61.1 \mathrm{kDa}$, or alternatively, two AgAtg16 dimers, with a theoretical molecular weight of 76.5 $\mathrm{kDa}$. 


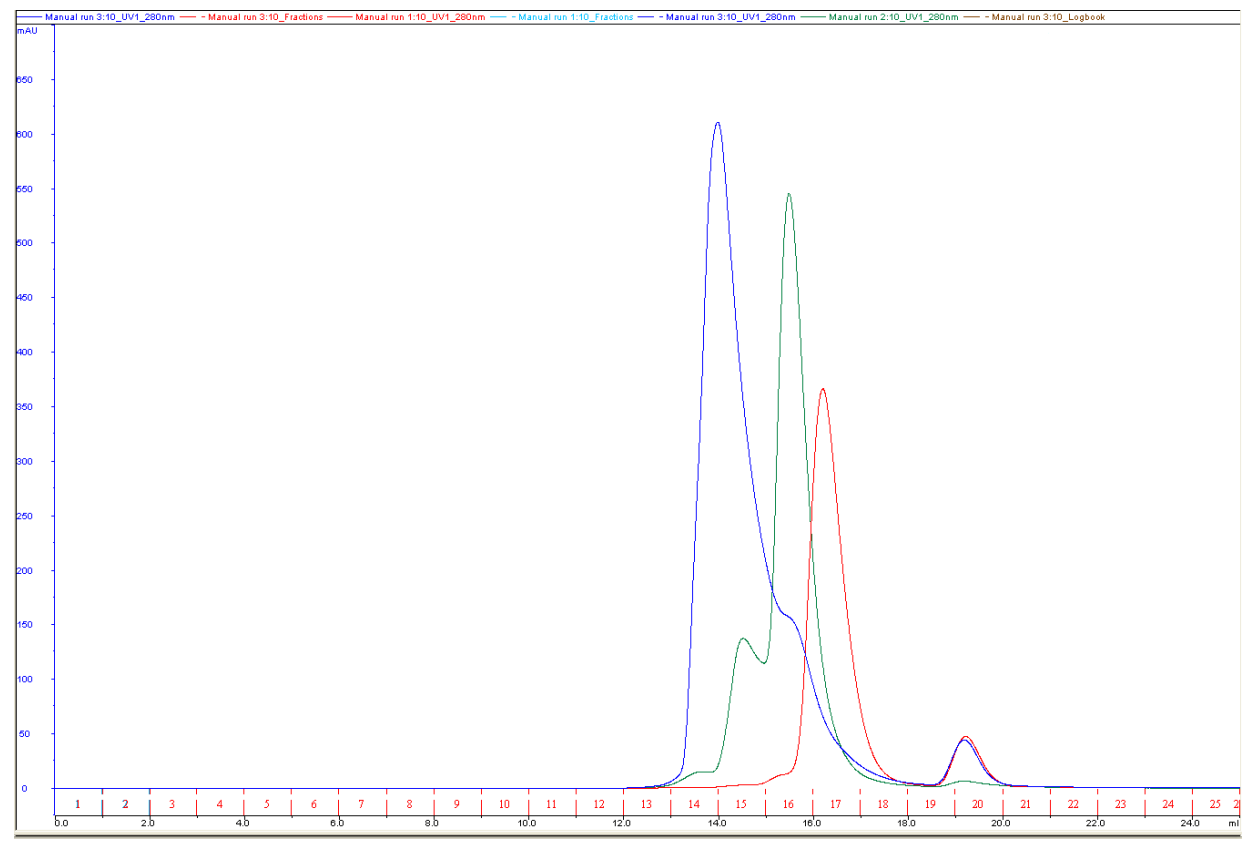

Figure 3.2.5.: SEC of KlAtg21, AgAtg16 (70-124) alone and KlAtg21-AgAtg16 (70-124) complex. Chromatograms of size exclusion chromatography using a Superdex 200 10/300 GL column prior to MALLS measurements. Green curve: KlAtg21, Red curve: AgAtg16 (70-124), blue curve: KlAtg21AgAtg16 (70-124) complex.

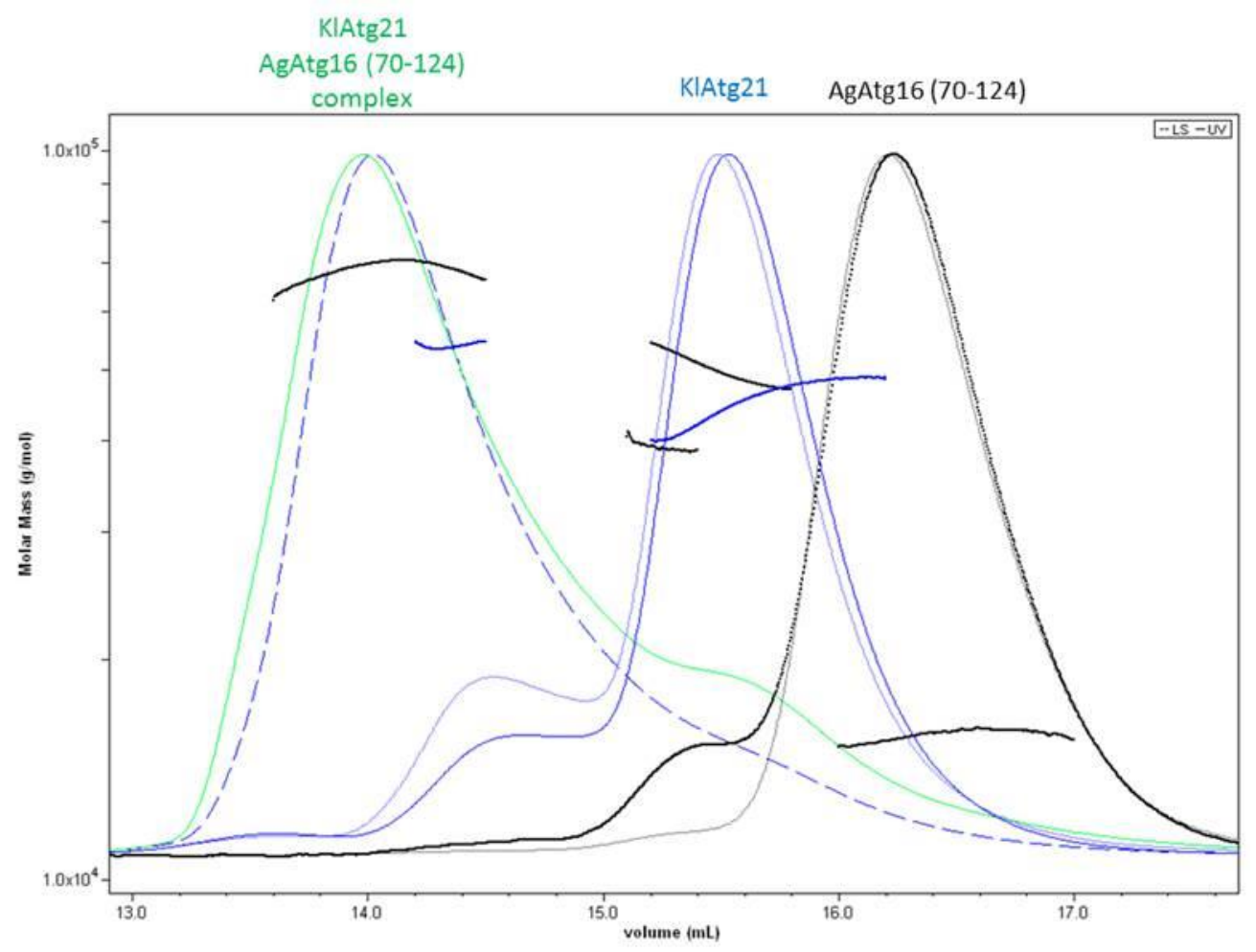

Figure 3.2.6.: MALLS measurements of KIAtg21, AgAtg16 (70-124) and KlAtg21-AgAtg16 (70124) complex. Peaks correspond to size exclusion chromatography elution volume ( $x$-axis). Horizontal lines indicate molar mass of the corresponding SEC peak (y-axis). 
Table 3.2.1.: Molecular weights of KIAtg21, AgAtg16 (70-124) and KlAtg21-AgAtg16 (70-124) complex. Listed are the average molar mass measured by SEC-MALLS and the theoretical mass calculated from the protein sequence including Tags.

\begin{tabular}{|c|c|c|c|}
\hline Protein & $\begin{array}{c}\text { AgAtg16 (70-124) } \\
\text { dimer }\end{array}$ & KlAtg21 & $\begin{array}{c}\text { KIAtg21- } \\
\text { AgAtg16(70-124) } \\
\text { complex }\end{array}$ \\
\hline $\begin{array}{c}\text { Average molecular } \\
\text { weight (g/mol) }\end{array}$ & $1.574 \times 10^{4}( \pm 0.043 \%)$ & $4.314 \times 10^{4}( \pm 0.009 \%)$ & $6.767 \times 10^{4}( \pm 0.009 \%)$ \\
\hline $\begin{array}{c}\text { Theoretical molecular } \\
\text { weight (g/mol) }\end{array}$ & $1.54 \times 10^{4}$ & $4.57 \times 10^{4}$ & unknown \\
\hline
\end{tabular}

\subsubsection{Isothermal calorimetry measurements of the KIAtg21-AgAtg16 complex}

In order to investigate the thermodynamic parameters of the KlAtg21- AgAtg16 complex formation isothermal titration calorimetry (ITC) measurements were done together with Dr. Ángel Pérez-Lara (Department for Neurobiology). $200 \mu \mathrm{M}$ AgAtg16(40-124) or (70-124) were titrated in the cell containing $20 \mu \mathrm{M}$ KlAtg21. An exothermic reaction indicating binding was not observed.

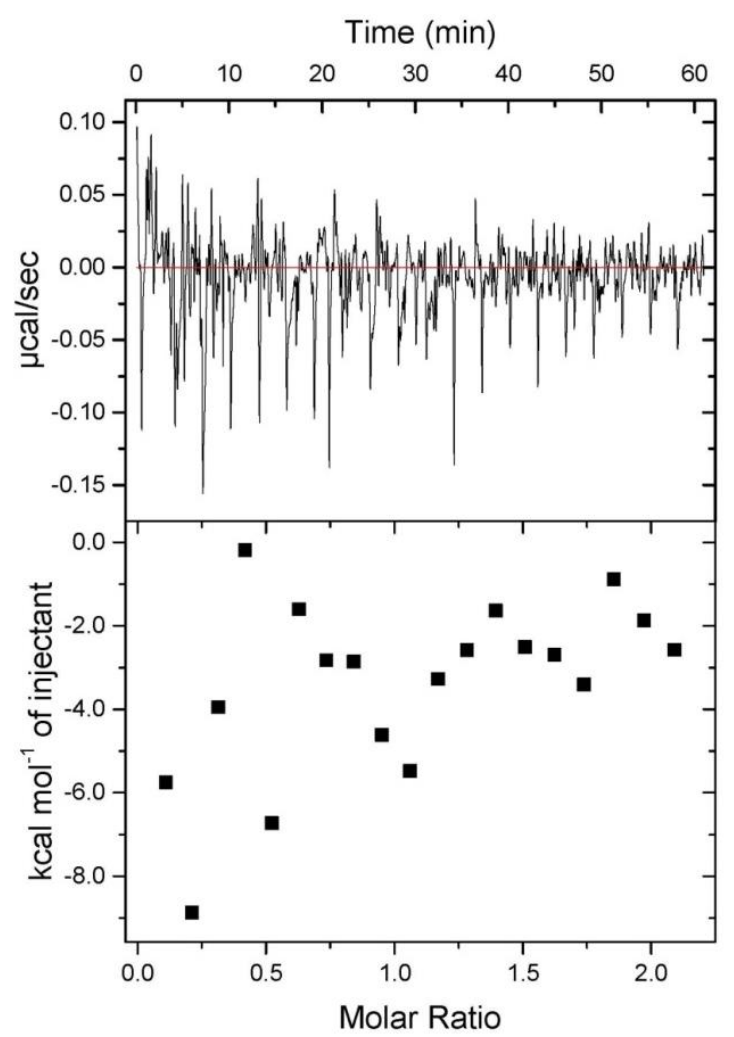

Figure 3.2.7.: Isothermal titration calorimetry titration curves of KIAtg21 with AgAtg16(40124). $200 \mu \mathrm{M}$ AgAtg16 (40-124) was titrated into $20 \mu \mathrm{M}$ KlAtg21. Data was fitted with a One Set of Sites fitting model. Top panel shows raw ITC data. Bottom panel shows integrated heat. 


\subsubsection{Structure determination of the KlAtg21-AgAtg16 complex}

Structure determination of the Atg21-Atg16 complex was a main goal in our group. Dr. Karin Kühnel crystallized the KlAtg21-AgAtg16 (40-124) complex by in-situ proteolysis with 1:1000 Clostripain and a precipitant composed of $15 \%(\mathrm{w} / \mathrm{v})$ PEG 4000 and 0.1 M HEPES pH 7.0. Crystals were optimized but their diffraction did not improve. Structure determination of the KlAtg21-AgAtg16 complex was performed by me from the point of data collection.

\subsubsection{X-ray data collection and processing for the KIAtg21-AgAtg16 complex}

Data were collected at $100 \mathrm{~K}$ at beamline X06SA (Swiss Light Source, Paul Scherrer Institute, Villigen, Switzerland). Because K1Atg21-AgAtg16(40-124) crystals had an elongated shape a grid screening was performed for every crystals tested. Grid screen analysis revealed that all crystals displayed different diffraction properties sidelong (Fig. 3.2.8.). The best diffracting region was chosen to collect data.

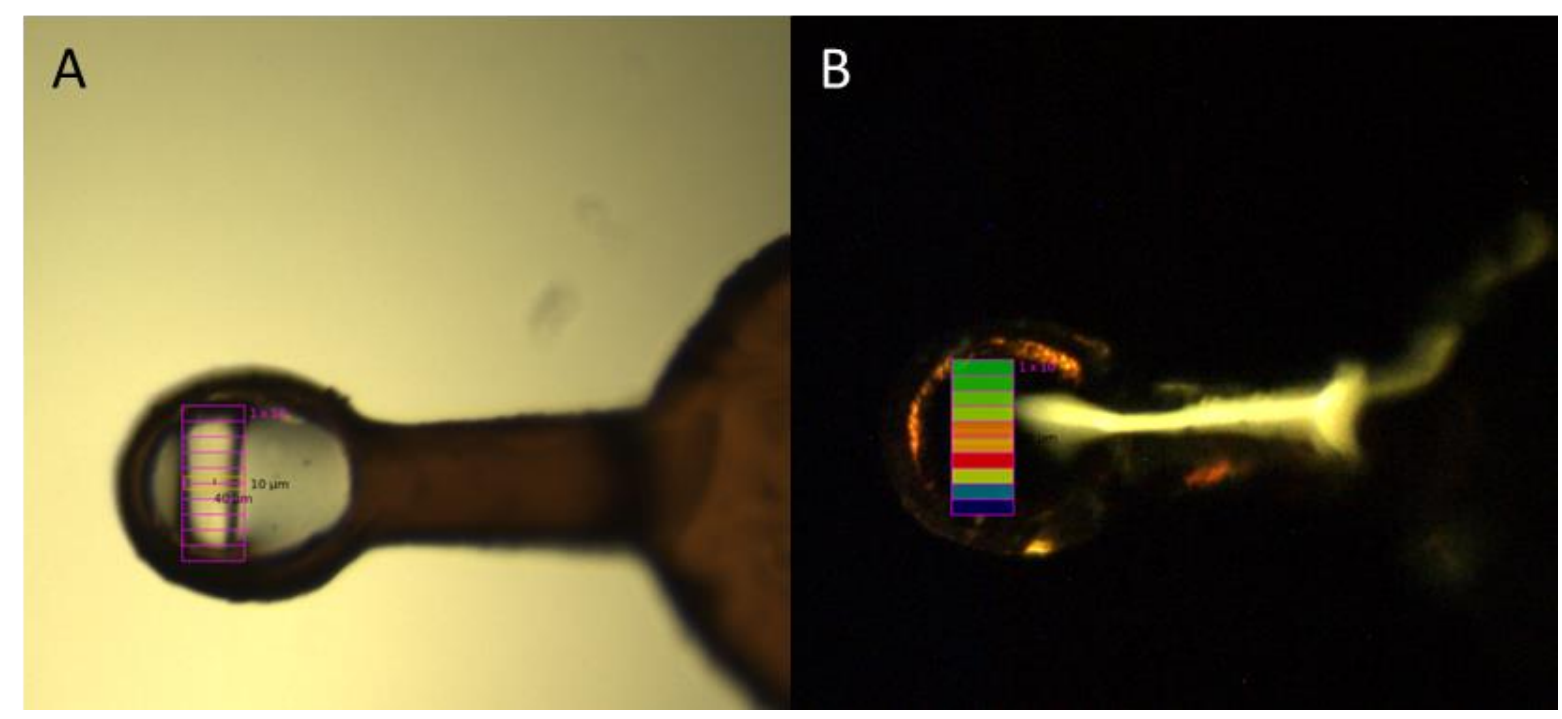

Figure 3.2.8.: Grid screen of a KlAtg21-AgAtg16 (40-124) crystal. A: Crystal mounted at PXI at the Swiss Light Source. Pink squares define zone for grid screen. Each square has the size of $10 \times 40$ $\mu \mathrm{m}$. B: Results of grid screening. Colors indicate the diffraction intensity from low (dark blue) to high (red).

Four test shots were taken at the best position to index the crystal. A data collection strategy was determined with go.com (local software written by Dr. M. Wang, SLS). A complete native data set at $4.0 \AA$ resolution was recorded with an oscillation angle of 0.5 over $360^{\circ}$ rotation. Table 3.2.2. summarizes the data collection parameters. 
Table 3.2.2.: Data collection for the KIAtg21-AgAtg16(40-124) complex

\begin{tabular}{|l|l|}
\hline Beamline & X06SA, Swiss Light Source (Switzerland) \\
\hline Detector & EIGER 16M (Dectris) \\
\hline Detector distance & $600 \mathrm{~mm}$ \\
\hline$\varphi / \Delta \varphi$ & $0.2 / 360^{\circ}$ \\
\hline Exposure time & $0.1 \mathrm{sec}$ \\
\hline Beam intensity & 0.1 \\
\hline$\lambda$ & $1 \AA$ \\
\hline Number of frames & 1800 \\
\hline
\end{tabular}

Data were processed with input values specific for the EIGER 16M detector using the XDS software package. KlAtg21-AgAtg16 crystals belong to space group P3221 (154) with unit cell dimensions of $\mathrm{a}=123.5 \AA, \mathrm{b}=123.5 \AA, \mathrm{c}=185.22 \AA, \alpha=90^{\circ}, \beta=90^{\circ}, \gamma=120^{\circ}$. XDSCONV was used to convert scaled data to unmerged CCP4F format.

\subsubsection{Molecular replacement for the KIAtg21-AgAtg16 complex}

The Phenix program suite was used for molecular replacement and refinement. The first step of structure solution was to try molecular replacement (MR) with the known PROPPIN structures of Kluyveromyces marxianus Hsv2 (PDB code: 4EXV, [9]), Kluyveromyces lactis Hsv2 (PDB code: 4V16, [19]) and Pichia angusta Atg18 (PDB code: 5LTD and 5LTG, [119]). Flexible loop regions were truncated from the search models first. Loop 318-229 was removed for KlHsv2 (14-338) and the corresponding loop 330-339 was deleted in KmHsv2 (19-350). The two available structures of PaAtg18 were superimposed and loop 91-97 was truncated. Additionally, side chains were deleted for all models. Molecular replacement with truncated PaAtg18 and KmHsv2 was successful (Table 3.2.3.). Two PROPPIN molecules were placed and the same orientation of the $\beta$-propellers was observed for PaAtg18 and KmHsv2 (Fig. 3.2.9.). However, MR with KlHsv2 did not give a solution. Using PaAtg18 as a search model yielded a better molecular replacement solution and was used for the final KlAtg21-AgAtg16 model. 

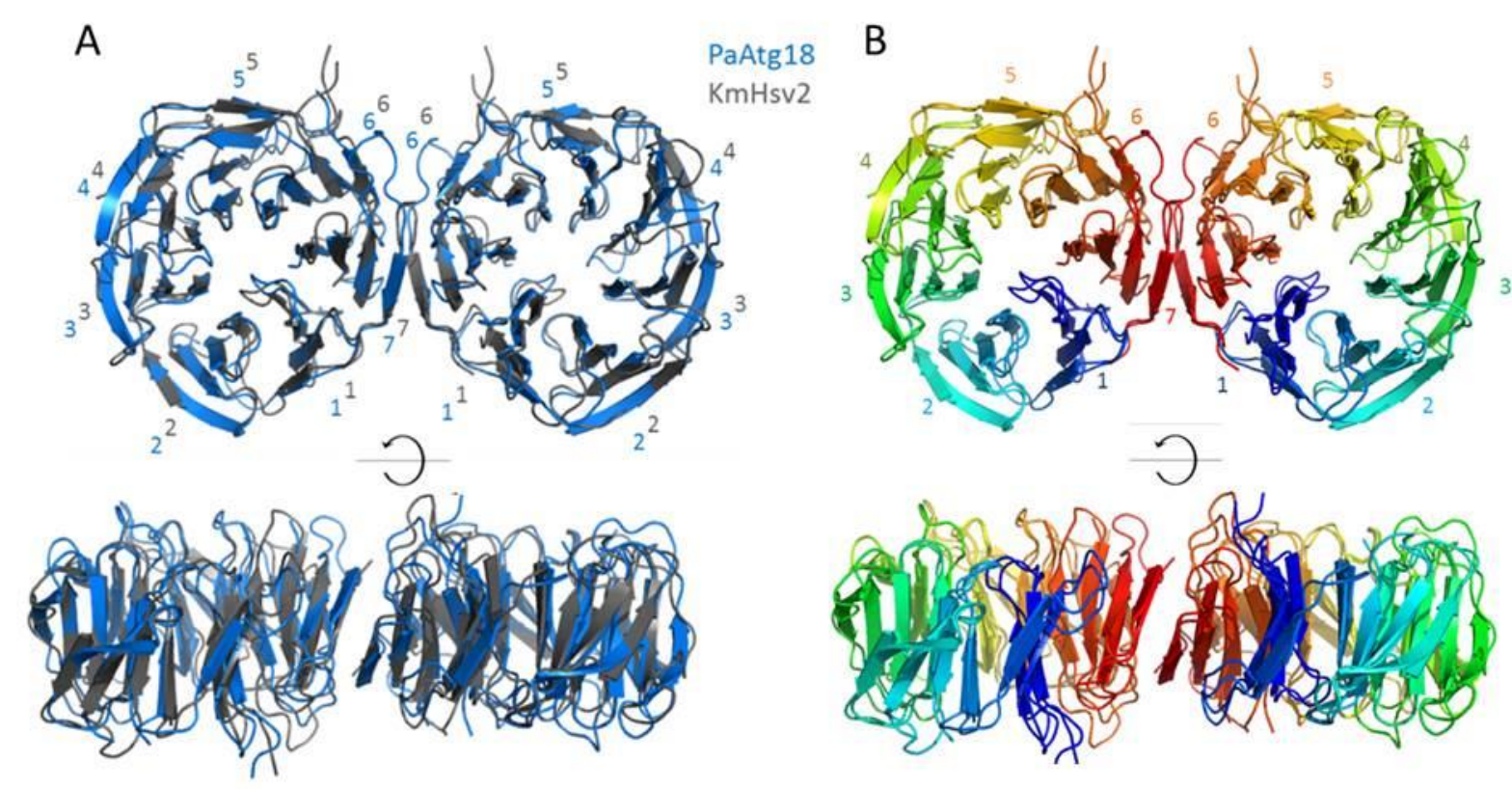

Figure 3.2.9.: First round in the molecular replacement structure determination of KIAtg21AgAtg16. Two PROPPIN molecules were placed in the asymmetric unit. Overlay of cartoon representations. A: Blue: MR solution with PaAtg18 as search model (Superimposition of PDB codes 5LTD and 5LTG), Grey: MR solution using KmHsv2 as a search model (PDB code: 4EXV). B: Both solutions rainbow colored from blue at the $\mathrm{N}$-terminus to red at the $\mathrm{C}$-terminus. Numbers indicate propeller.

Table 3.2.3.: Result of first round of molecular replacement with Phaser_MR for the KIAtg21AgAtg16 complex structure. Using PaAtg18 (superimposed and truncated) or KmHsv2 (truncated) as polyalanine search models for KlAtg21.

\begin{tabular}{|c|c|c|c|c|}
\hline Search model & Component copies & Number of MR solutions & Top LLG & Top TFZ \\
\hline PaAtg18 & 2 & 1 & 1268.2 & 37.7 \\
\hline KmHsv2 & 2 & 1 & 547.6 & 23.7 \\
\hline
\end{tabular}

Once the two PROPPIN molecules were placed in the electron density a first round of refinement clearly revealed additional electron density between the two molecules with a helical shape and a length of around $48 \AA$ (Fig. 3.2.10 A). A second step of molecular replacement with the short dimeric coiled coil domain of ScAtg16 (PDB code: 3A7P) and the dimeric coiled coil domain of AgAtg16 (see section 3.2.6.) was then performed. Both molecules gave good solutions for both solutions from the first round of MR (Table 3.2.4.). Both Atg16 dimer gave a perfect fit in the additional electron density (Fig. 3.2.10 B). Conserved residues were placed at the same position for both Atg16 structures. The Atg16 models were truncated at both ends to the length of accessible electron density. Because AgAtg16 (40-124) was used for crystallization the coiled coil domain of AgAtg16 was used for the final KlAtg21-AgAtg16 model. Due to the low resolution of the data (only $4.0 \AA$ ) side 
chains are not visible, so the sequence of KlAtg21 was not built in and proteins were included as polyalanine models. One cycle of refinement was performed for the polyalanine KlAtg21AgAtg16 model after molecular replacement to evaluate the solution. The $\mathrm{R}_{\text {work }} / \mathrm{R}_{\text {free }}$ values of $0.38 / 0.39$ show that the molecular replacement solution is correct. Data collection and refinement statistics are listed in Table 3.2.5 and a comparison with structures with a similar resolution limit is shown in (Fig. 3.2.11.).

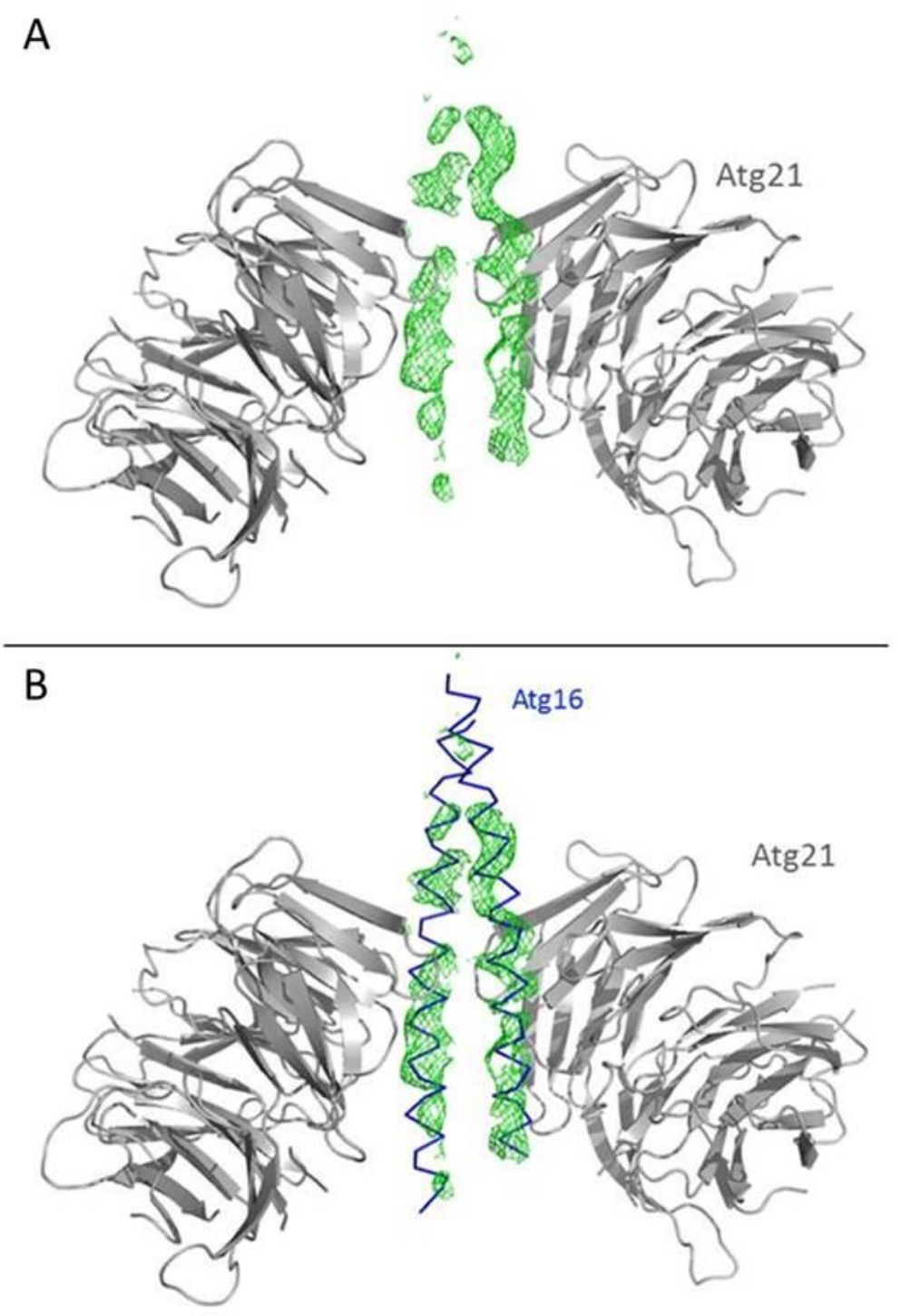

Figure 3.2.10.: First round of molecular replacement for Atg21 reveals additional electron density. Cartoon representation of two PROPPIN molecules in grey. A: Omit Fo-Fc electron density map contoured at $2 \sigma$ in green showed an additional helical shaped electron density. B: Superimposed Atg16 coiled coil domain (blue ribbon presentation). 
Table 3.2.4.: Result of second round of molecular replacement with Phaser_MR for the KlAtg21-AgAtg16 complex structure. Using truncated ScAtg16 dimer (PDB code: 3A7O) or truncated AgAtg16 dimer as polyalanine search models for AgAtg16.

\begin{tabular}{|c|c|c|c|c|c|}
\hline $\begin{array}{c}\text { Fixed partial } \\
\text { solution }\end{array}$ & $\begin{array}{c}\text { Search } \\
\text { model }\end{array}$ & $\begin{array}{c}\text { Component } \\
\text { copies }\end{array}$ & $\begin{array}{c}\text { Number of MR } \\
\text { solutions }\end{array}$ & $\begin{array}{c}\text { Top } \\
\text { LLG }\end{array}$ & $\begin{array}{c}\text { Top } \\
\text { TFZ }\end{array}$ \\
\hline PaAtg18 & ScAtg16 & 1 & 13 & 1291.7 & 14.7 \\
\hline PaAtg18 & AgAtg16 & 1 & 11 & 1308.9 & 15.7 \\
\hline KmHsv2 & ScAtg16 & 1 & 15 & 622.8 & 13.9 \\
\hline KmHsv2 & AgAtg16 & 1 & 10 & 628.7 & 14.2 \\
\hline
\end{tabular}

Table 3.2.5.: Data collection and refinement statistics for the KlAtg21-AgAtg16(40-124) complex. Values in parentheses refer to the highest resolution shell, Ramachandran statistics were calculated with Molprobity.

\begin{tabular}{|c|c|}
\hline $\begin{array}{l}\text { Data collection } \\
\text { Space group }\end{array}$ & $\mathrm{P} 3_{2} 21$ \\
\hline $\begin{array}{l}\text { Cell dimensions } \\
\text { a, b, c }(\AA) \\
\alpha, \beta, \gamma\left({ }^{\circ}\right)\end{array}$ & $\begin{array}{l}123.5,123.5,185.22 \\
90.0,90.0, \quad 120.0\end{array}$ \\
\hline $\begin{array}{l}\text { Resolution range }(\AA) \\
\text { Total reflections } \\
\text { Unique reflections } \\
\text { Multiplicity } \\
\text { Completeness }(\%) \\
\text { Mean } \mathrm{I} / \sigma \\
\text { Wilson B factor }\left(\AA^{2}\right) \\
\mathrm{R}_{\text {meas }}(\%) \\
\mathrm{CC}_{1 / 2}\end{array}$ & $\begin{array}{l}46.3-4.0(4.15-4.0) \\
144202(14510) \\
14241(1426) \\
10.1 \\
99.6(98.7) \\
12.6(1.8) \\
148.3(129.2) \\
15.5(159.9) \\
99.9(73.6)\end{array}$ \\
\hline $\begin{array}{l}\text { Refinement } \\
\mathrm{R}_{\text {work }} \\
\mathrm{R}_{\text {free }} \\
\end{array}$ & $\begin{array}{l}0.386(0.454) \\
0.397(0.504)\end{array}$ \\
\hline Molecules/AU & 4 \\
\hline $\begin{array}{l}\text { B-factors }\left(\AA^{2}\right) \\
\text { Structure validation } \\
\text { Ramachandran favored }(\%) \\
\text { Ramachandran allowed }(\%) \\
\text { Ramachandran outliers }(\%) \\
\text { Rotamer outliers }(\%) \\
\text { Clashscore }\end{array}$ & $\begin{array}{l}47.5 \\
86 \\
11 \\
2.8 \\
0 \\
6.75\end{array}$ \\
\hline $\begin{array}{l}\text { RMSD deviations } \\
\text { Bond lengths }(\AA) \\
\text { Bond angles }\left({ }^{\circ}\right)\end{array}$ & $\begin{array}{l}0.002 \\
0.63\end{array}$ \\
\hline
\end{tabular}




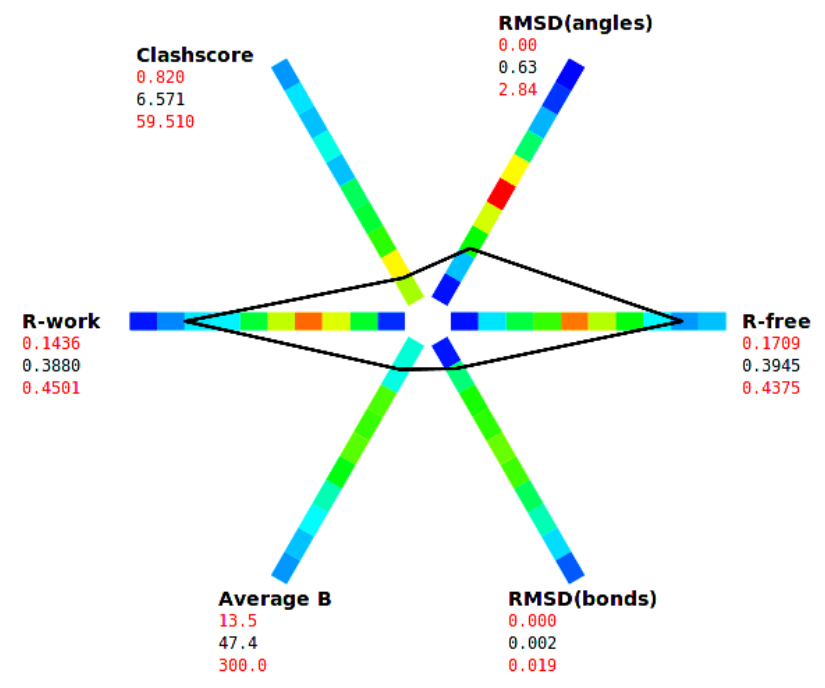

Figure 3.2.11.: Refinement statistic plots for the KlAtg21-AgAtg16(40-124) complex. Polyglon plot. Structure statistics (black numbers) are compared to PDB entries with similar resolution (red numbers). Plot was made with phenix.refine.

\subsubsection{Structure based comparison of yeast PROPPINs}

Although molecular replacement for KlAtg21-AgAtg16 gave consistent models with reasonable refinement statistics using all combinations of PaAtg18, KmHsv2, ScAtg16 (60118) and AgAtg16 (43-108) as search models, further proof of the model was done due to the low resolution of the structure. The protein sequence of KlAtg21 was aligned with other yeast PROPPINs, incorporating the known PaAtg18, KmHsv2 and KlHsv2 structures using $\mathrm{T}$ Coffee Expresso [5] (Fig. 3.2.12.). 
A

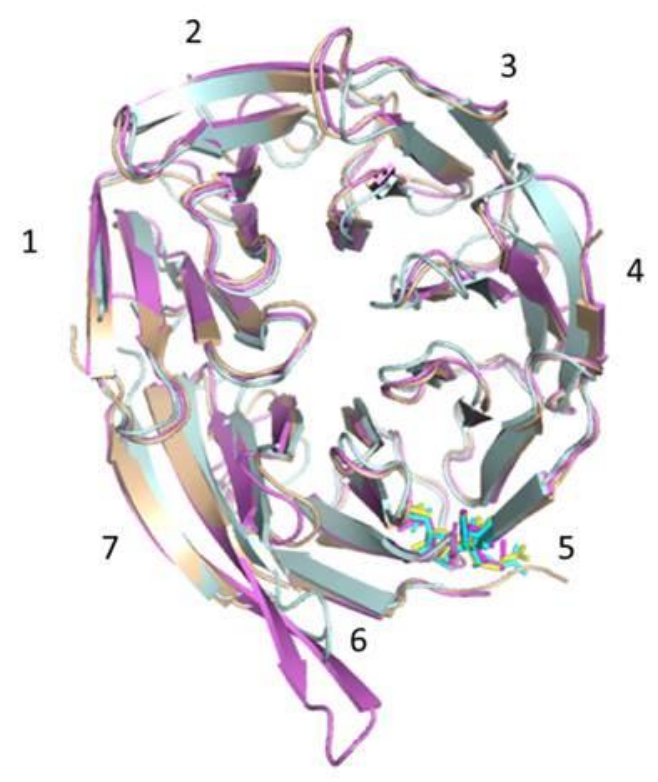

B

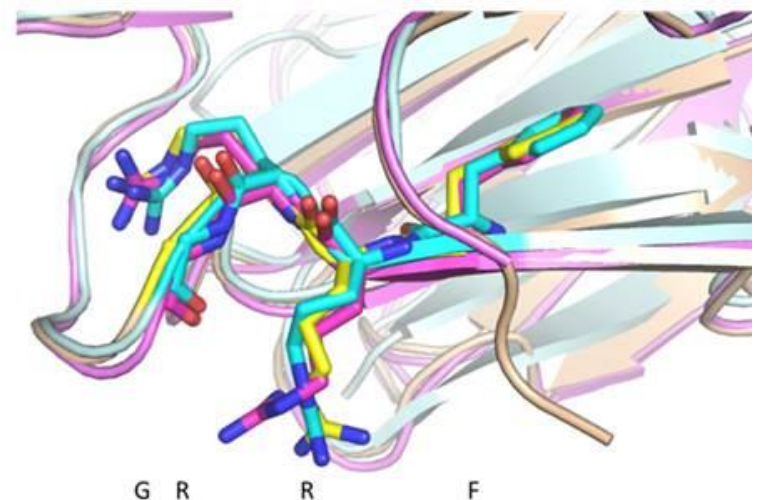

G R

$\mathrm{C}$

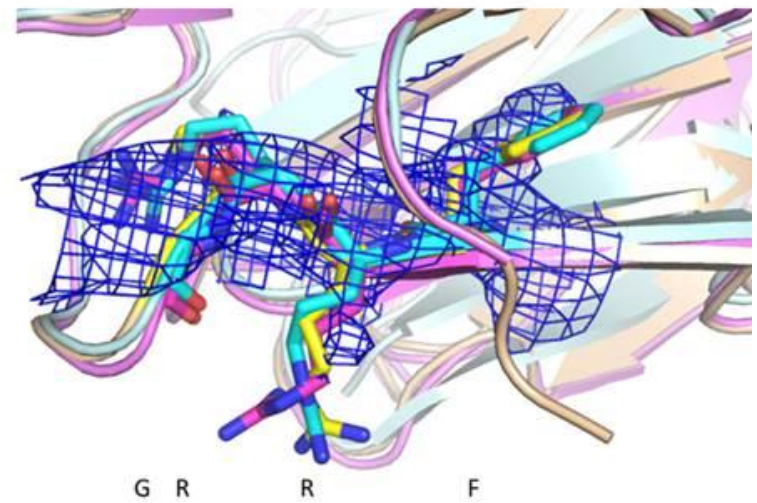

Figure 3.2.12.: Superimposition of PaAtg18, KIHsv2 and KmHsv2. Cartoon representation of PaAtg18 in light turquoise, KlHsv2 in light pink and KmHsv2 in light yellow. A: Overview of superimposed PROPPIN structures. Numbers represent propeller blades one to seven. B: Close up view of the phosphoinositide binding motif "FRRG". Residues FRRG presented as sticks. C: KlAtg21-AgAtg16 2mFo-DFc electron density map countered at $\sigma=1$ around the FRRG motif.

The blades in all three PROPPIN structures are conserved; only blade seven is less conserved. Especially, blades one to three, five and six are highly conserved, which includes the connecting loops (Fig. 3.2.13.). Superimposition of the structures verifies the alignment (Fig. 3.2.14. A). Functionally important regions like the phosphoinositide binding "FRRG" motif are conserved and the three structures are very similar in this region (Fig. 3.2.14. B).

To gain insights into the evolutionary conservation of amino acid positions based on the phylogenetic relations between homologous sequences in respect to the PaAtg18 and KmHsv2 structures ConSurf [44] was used. Yeast Atg21 sequences were aligned with either PaAtg18 or KmHsv2 using T Coffee Expresso. Multiple sequence alignments were done to calculate the amino acid conservation score. Of notice, besides a good overall conservation score the binding region of Atg16 at blade two and three is highly conserved (Fig. 3.2.13. and Fig. 3.2.14.). Especially, residues PaAtg18 R128 and KmHsv2 R105 that are homologous to the Atg16 binding site of K1Atg21 R103 are highly conserved. 
Blade 1

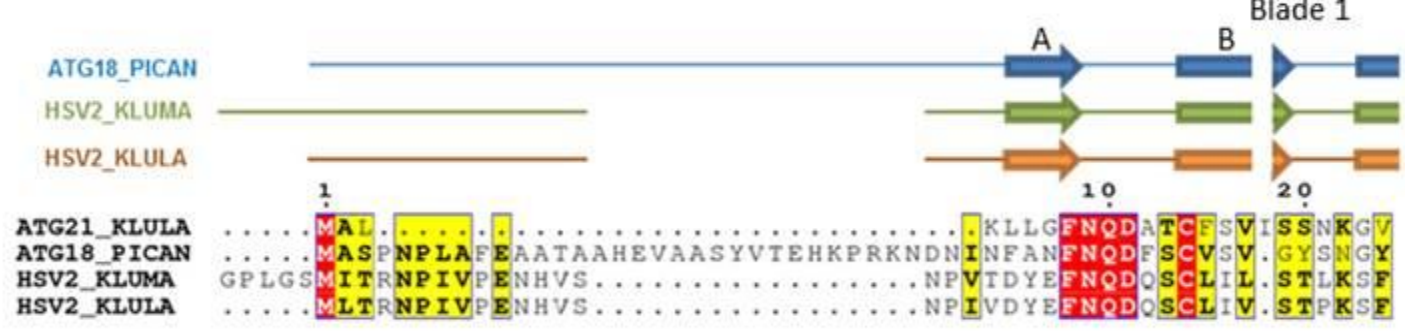

ATG18_PICAN HSV2 KLUMA

HSV2 KLULA
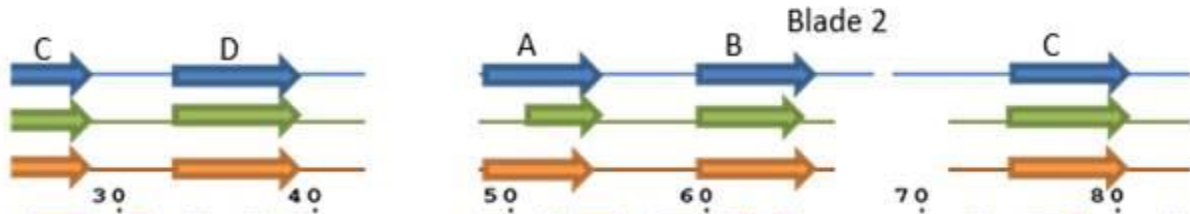

ATG21_KLULA ATG18_PICAN HSV2_KLUMA HSV2 KLULA

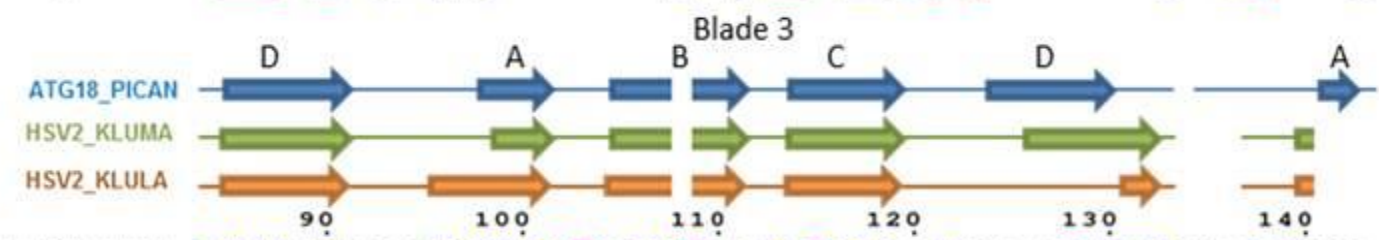

ATG21_KLULA KATICE立TEPHEIMDVIMNRKIICVVILSDOTEVYDISCMKLLRTIDVRGEKLKSTSKFR ATG18_PICAN QTTICELTEPGAILAVKLNRERLV.VLLEETIYIYDINNMRLLHTIETPS. NPNGLIALS HSV2_KLUMA KODVSRIKVDAPVKDLELSREFIV.VSYGDVISVFKFGNPWKRITDDIRF...GGVC... HSV2_KLULA KODITRVKLDAAVKDLFLSREFIV.VSQGDVISIFKFGNPWNKITEDIKE...GGVC...
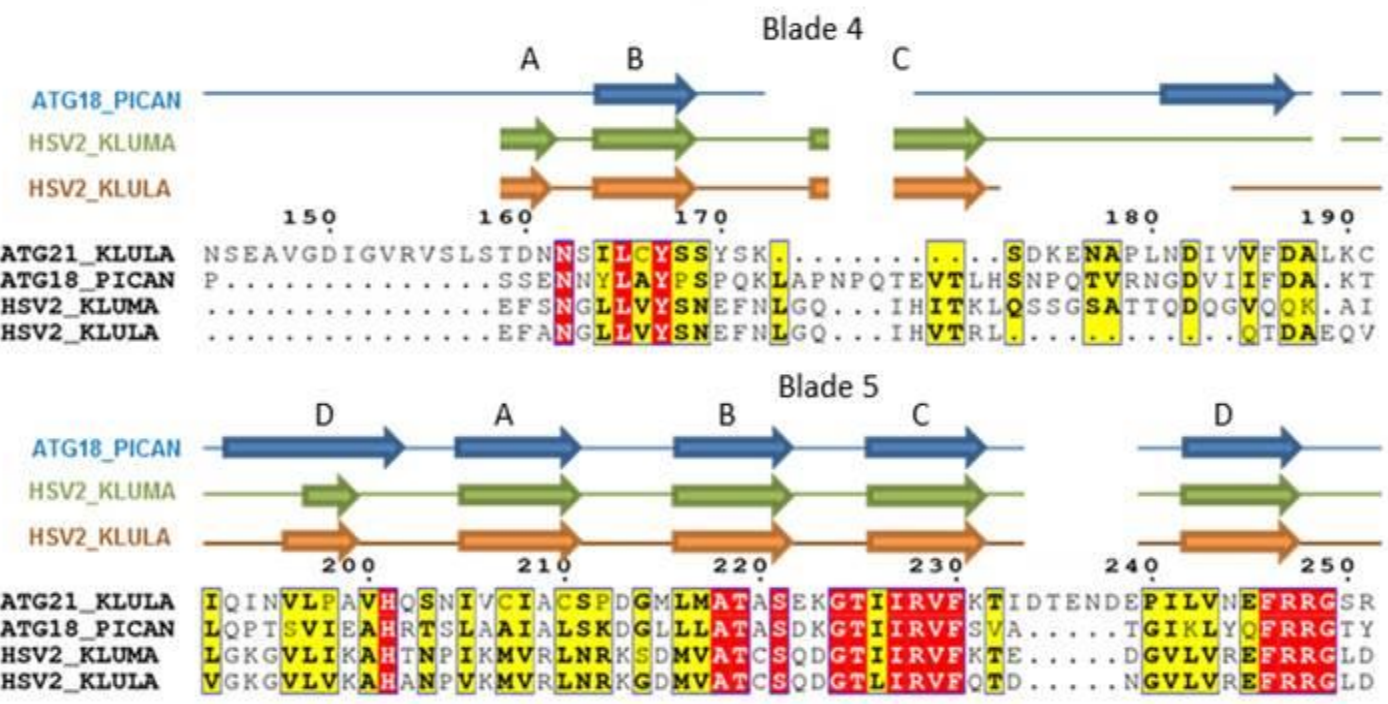

HSV2_KLULA VGKGVLVKAEANPVKMVRLNRKGDMVATCSODGTLIRVEOTD ..... NGVLVREERRGLD

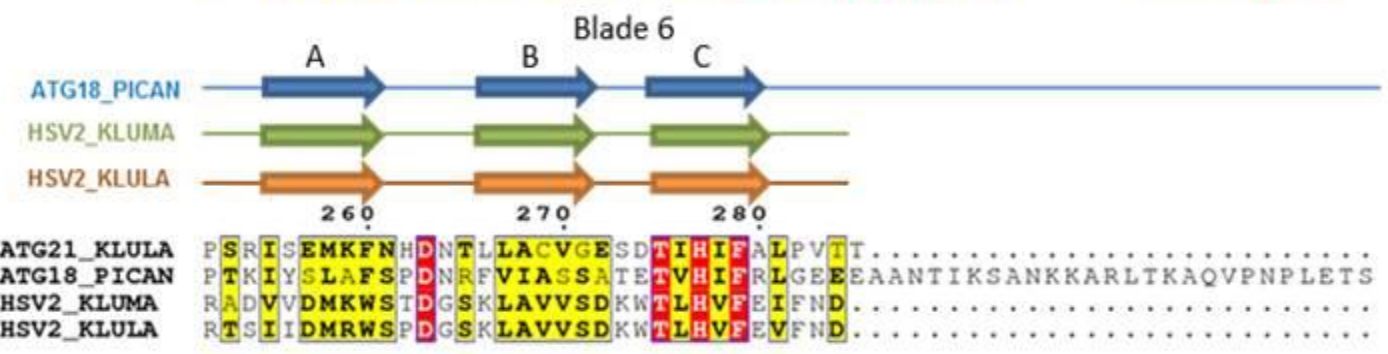

figure continues on next page 


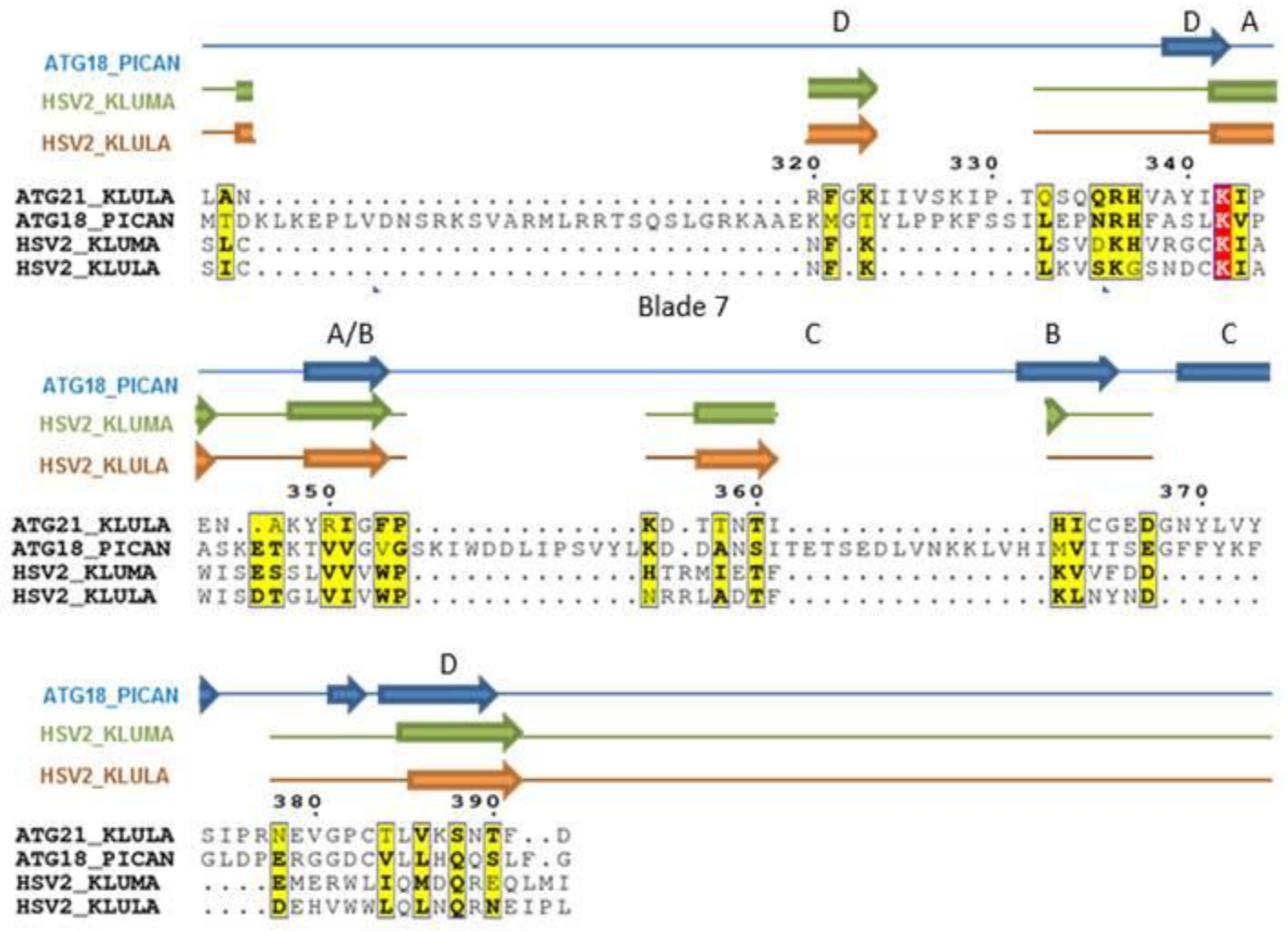

Figure 3.2.13.: Structure based sequence alignment of yeast PROPPINs. Similar residues are shown bold and yellow boxed, identical residues are colored white and red boxed. Secondary structure elements are marked. PROPPINS form a seven-bladed $\beta$-propeller scaffold (numbers 1-7) each blade consists of four antiparallel $\beta$-strands (letters A-D). Alignment was done with T-Coffee Expresso [5], Figure was made using ESPript 3.0 [116]. 


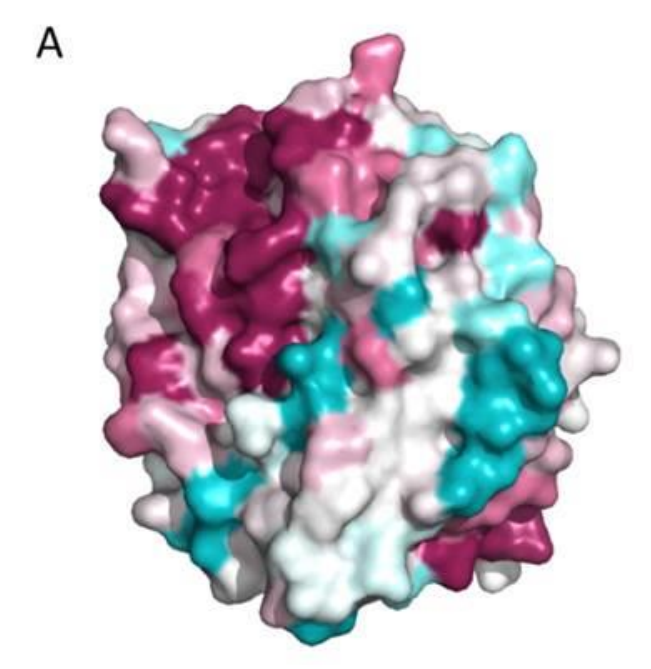

C

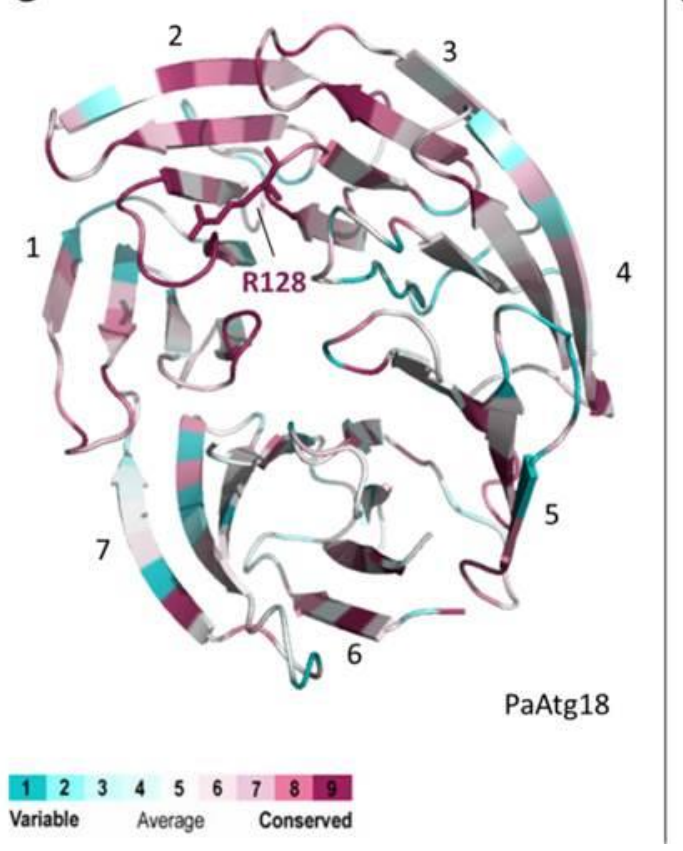

B

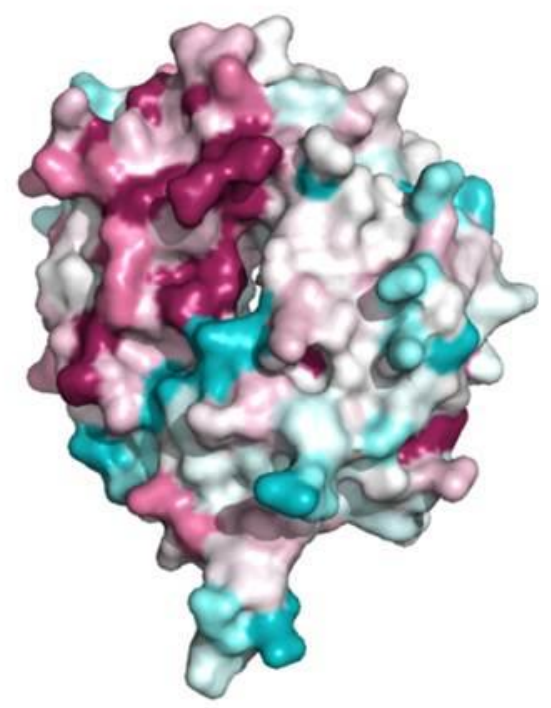

D

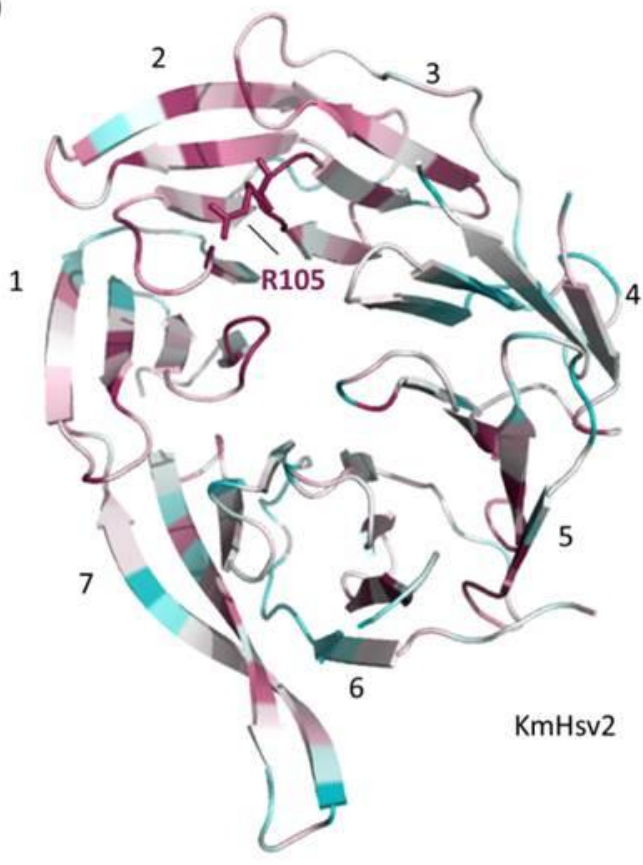

Figure 3.2.14.: ConSurf analysis of PaAtg18 and KmHsv2 for conserved Atg21 residues. Surface representation of A: PaAtg18 and $\mathbf{B}$ : KmHsv2. Cartoon representation of $\mathbf{C}$ : PaAtg18 and D: KmHsv2. Overview of superimposed PROPPIN structures. The amino acids are colored by their conservation grades as shown in the color-coding bar with turquoise-through-maroon indicating variable-through-conserved. An arginine important for Atg16 binding is presented as sticks in the cartoon representations. 


\subsubsection{Structure analysis of the KIAtg21-AgAtg16 complex}

Crystal packing of the KlAtg21-AgAtg16 complex is very loose, which might explain the weak diffraction of the crystals (Fig. 3.2.15.).

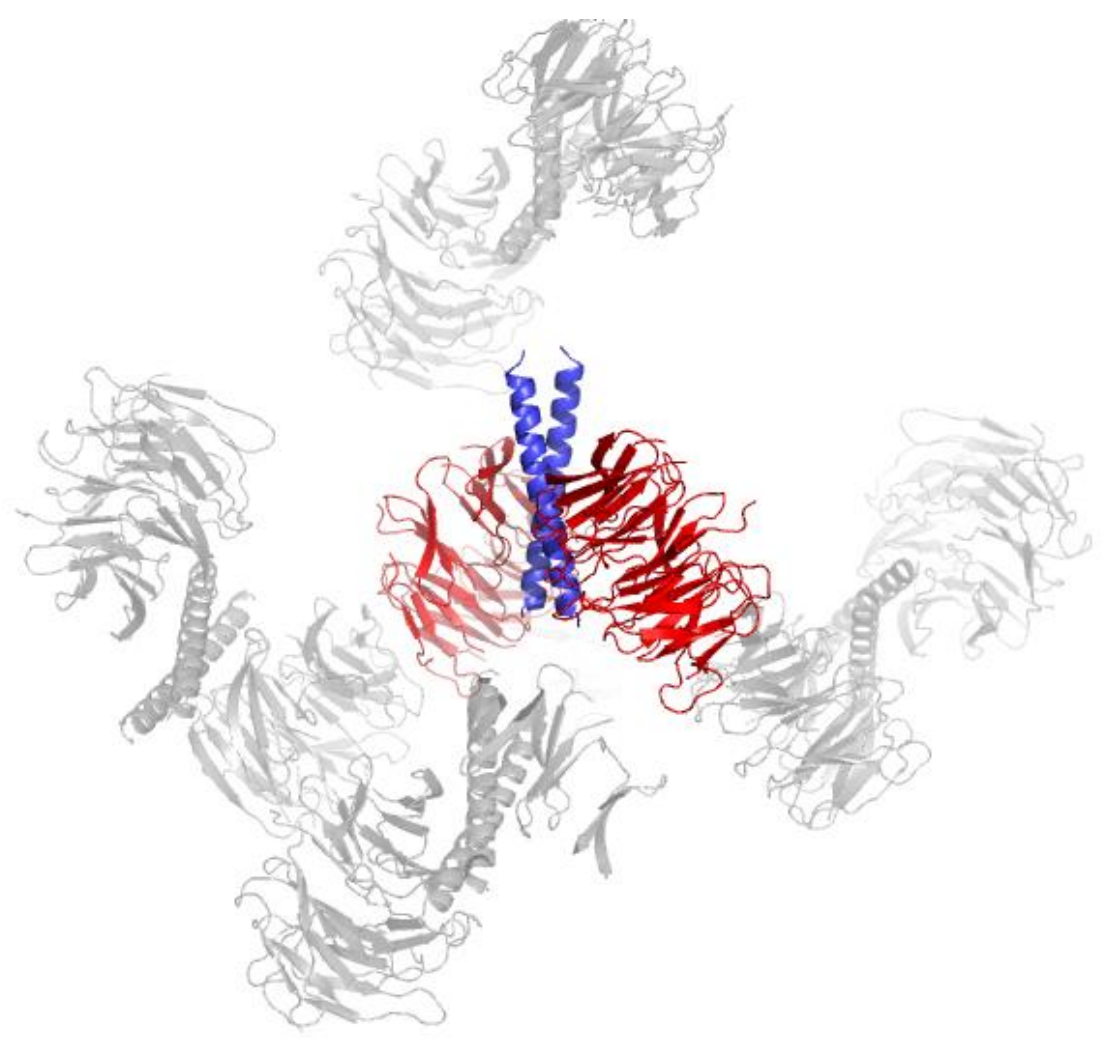

Figure 3.2.15.: Crystal packing in the KIAtg21-AgAtg16 crystal. One PROPPIN-Atg16 complex in the asymmetric unit is shown in color. Crystallographic symmetry-related molecules are shown in grey.

The crystal structure clearly shows the formation of a complex with two KlAtg21 molecules binding one AgAtg16 dimer, however molecular details of interaction cannot be observed due to the low resolution (4.0 $⿱$ ) of the electron density map (Fig.3.1.16.). The residues of the Atg16 molecule cannot build in with confidence because no side chains are visible at this low resolution, but molecular replacement localized both ScAtg16 and AgAtg16 structures in the same manner. Of notice, no SeMet crystals were obtained from the complex, which would have aided the sequence assignment. Nevertheless, important conclusions can be made about Atg21-Atg16 complex formation. 

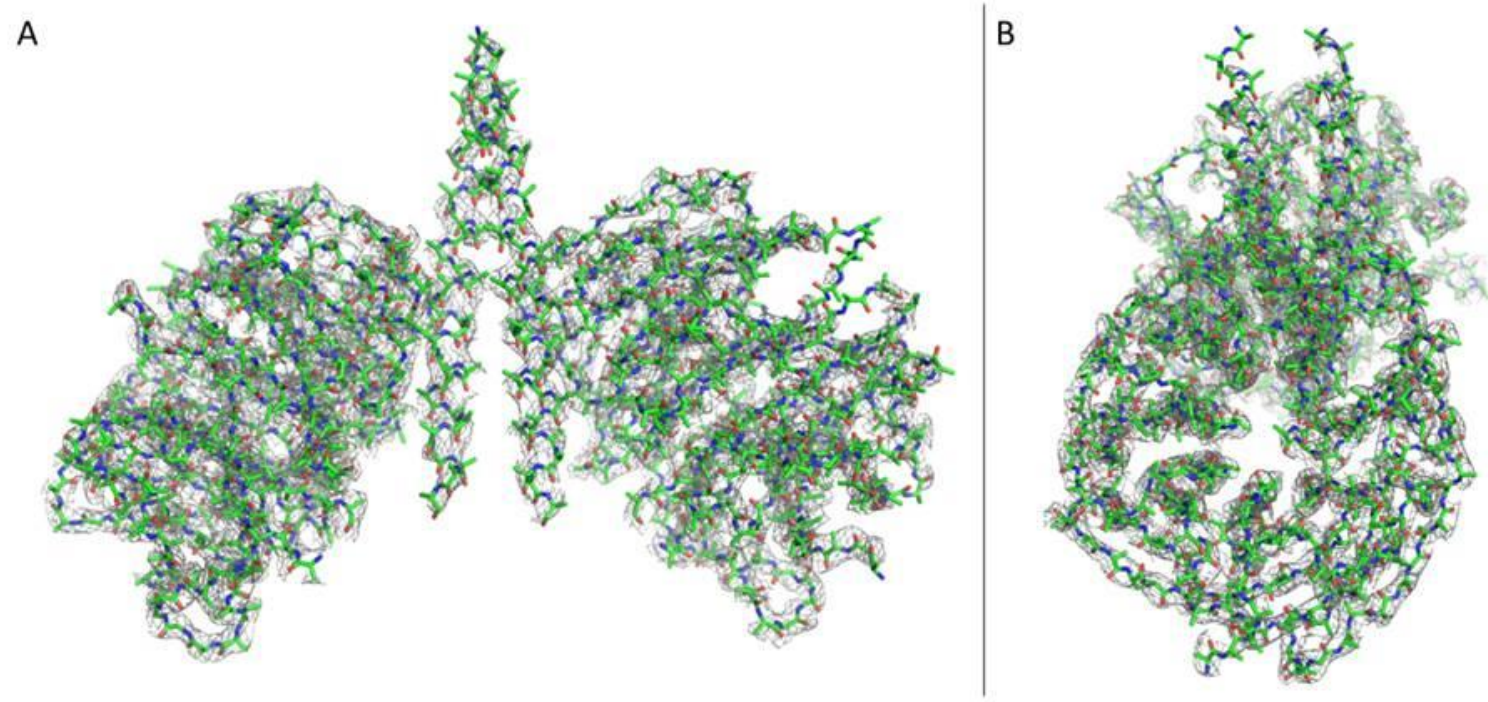

Figure 3.1.16.: Electron density map of the KlAtg21-AgAtg16 complex crystals. The $4.0 \AA$ resolution $2 \mathrm{mFo}-\mathrm{DFc}$ electron density map contoured at $\sigma=1$ in grey. The refined molecular replacement solution with the PaAtg18 and AgAtg16 structures are shown as poly-alanine models in stick representation.

KlAtg21 adopts the typical PROPPIN fold as seen in the electron density map (Fig. 3.2.16.). AgAtg16 binds to KlAtg21 blades two and three (Fig. 3.1.17.), which is opposite to the membrane binding motif FRRG on strand 5D and the loop connecting it with strand 6A. The two Atg21 molecules adopt a reversed V conformation to each other and give the impression to bend from the membrane towards the Atg16 dimer. According to this model residues K1Atg21 R103 and AgAg16 D78 come in close contact (Fig. 3.2.18.). The group of Prof. Michael Thumm recently proposed that ScAtg16 D101 and E102 of the coiled coil domain directly interact with Atg21 [58]. AgAtg16 D78 is syngeneic to ScAtg16 D101. To deepen the understanding of the interaction of Atg21 and Atg16 and to confirm the model presented here additional experiments were conducted in collaboration with the group of Prof. Michael Thumm. 


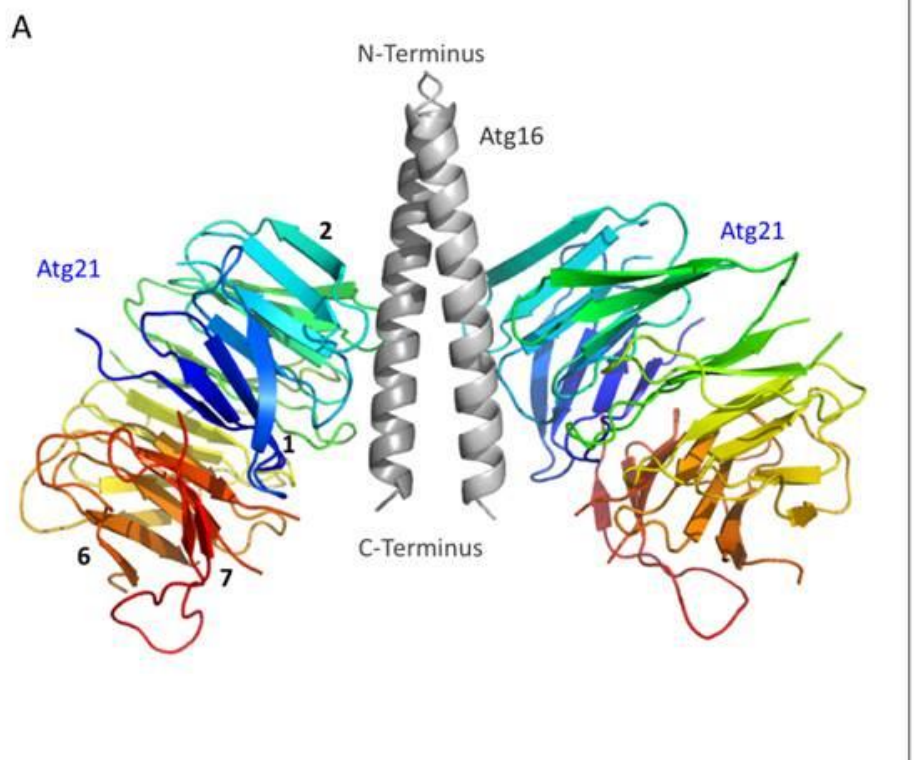

B

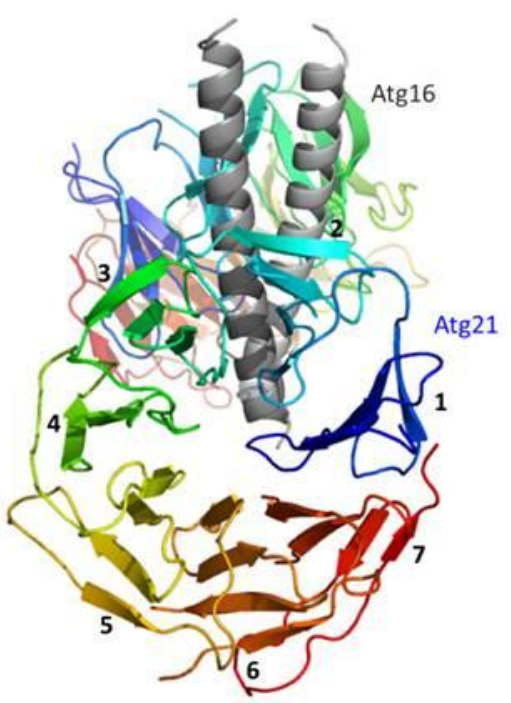

Figure 3.1.17.: Overview of the KIAtg21-AgAtg16 crystal structure. Two KlAtg21 molecules form a complex with the C-terminal part of the Atg16 coiled coil dimer. Interaction takes places between blade two and three of the KlAtg21 PROPPIN structure. Cartoon representation of KlAtg21 rainbow colored from blue at the $\mathrm{N}$-terminus to red at the C-terminus and AgAtg16 in grey. Numbers (1-7) correspond to the KlAtg21 PROPPIN blades.
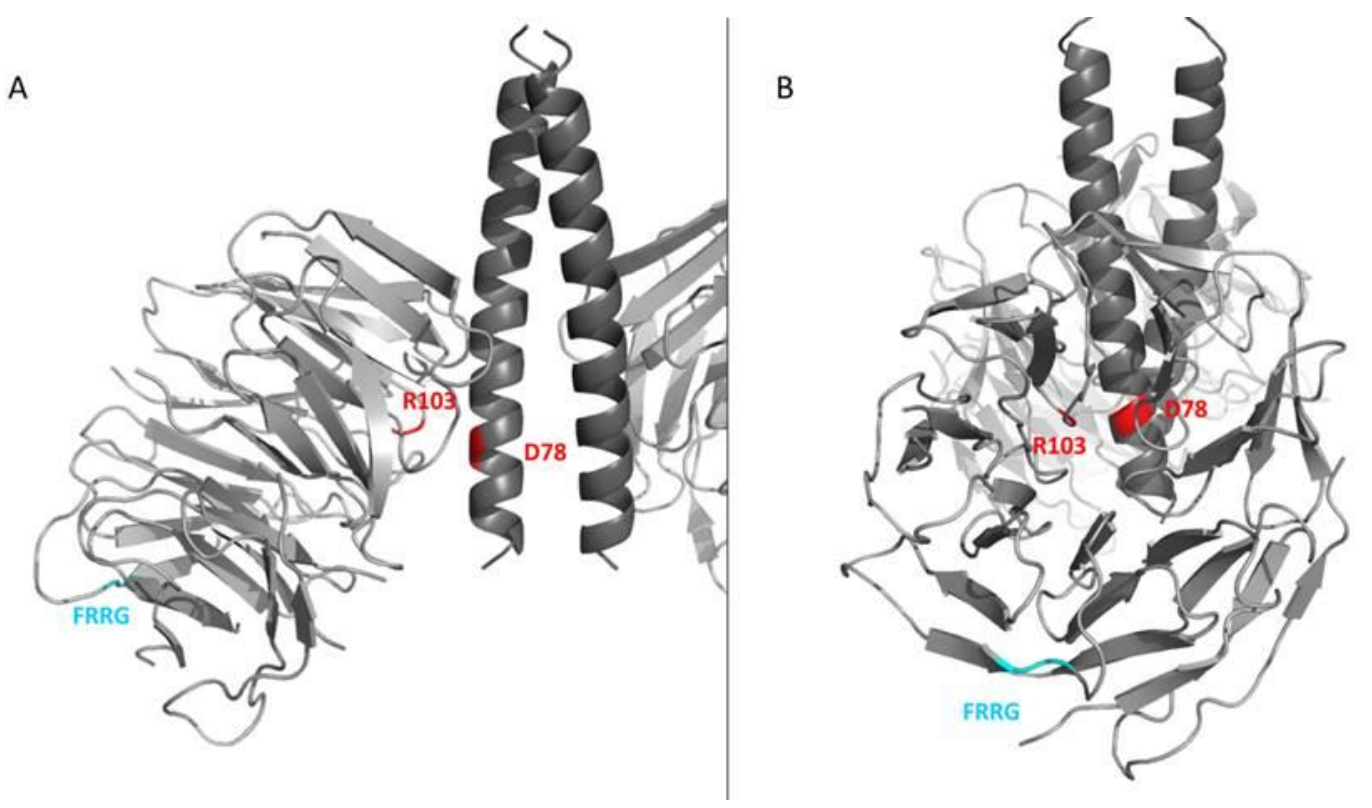

Figure 3.1.18.: Proposed model for KIAtg21-AgAtg16 interaction. Interaction of KlAtg21AgAtg16 probably occurs between residue AgAtg16 D78 and KlAtg21 R103 that is located opposite to the FRRG motif. Cartoon representation of KlAtg21 in grey and AgAtg16 in dark grey. 


\subsubsection{Identification of the KlAtg21 - AgAtg16 binding site by charge modification and analytic gel filtration analysis}

Upon structure determination of the KlAtg21-AgAtg16 complex and prior observations of the importance of ScAtg16 E102 and D101 for complex formation [58], Prof. Michael Thumm suggested the following KlAtg21 and AgAtg16 mutants to get further insights into Atg21Atg16 binding (Fig. 3.2.19.). Mutations reverse the charge of the residues that are likely to contribute to complex formation. Upon charge reversal, complex formation is probably restored. The KlAtg21 R103E and AgAtg16 (70-124) D78R mutants were purified and complex formation was studied by analytical gel filtration. As shown before complex formation was observed for KlAtg21 WT with AgAtg16 (70-124) WT (Fig. 3.2.20). No complex was formed using KlAtg21 WT and the mutant AgAtg16 (70-124) D78R (Fig. 3.2.21.) or the mutant K1Atg21 R103E and AgAtg16 WT (Fig. 3.2.22.), indicating their importance for complex formation. However, complex formation was not restored for Atg16 (70-124) D78R with KlAtg21 R103E in vitro (Fig. 3.2.23.).

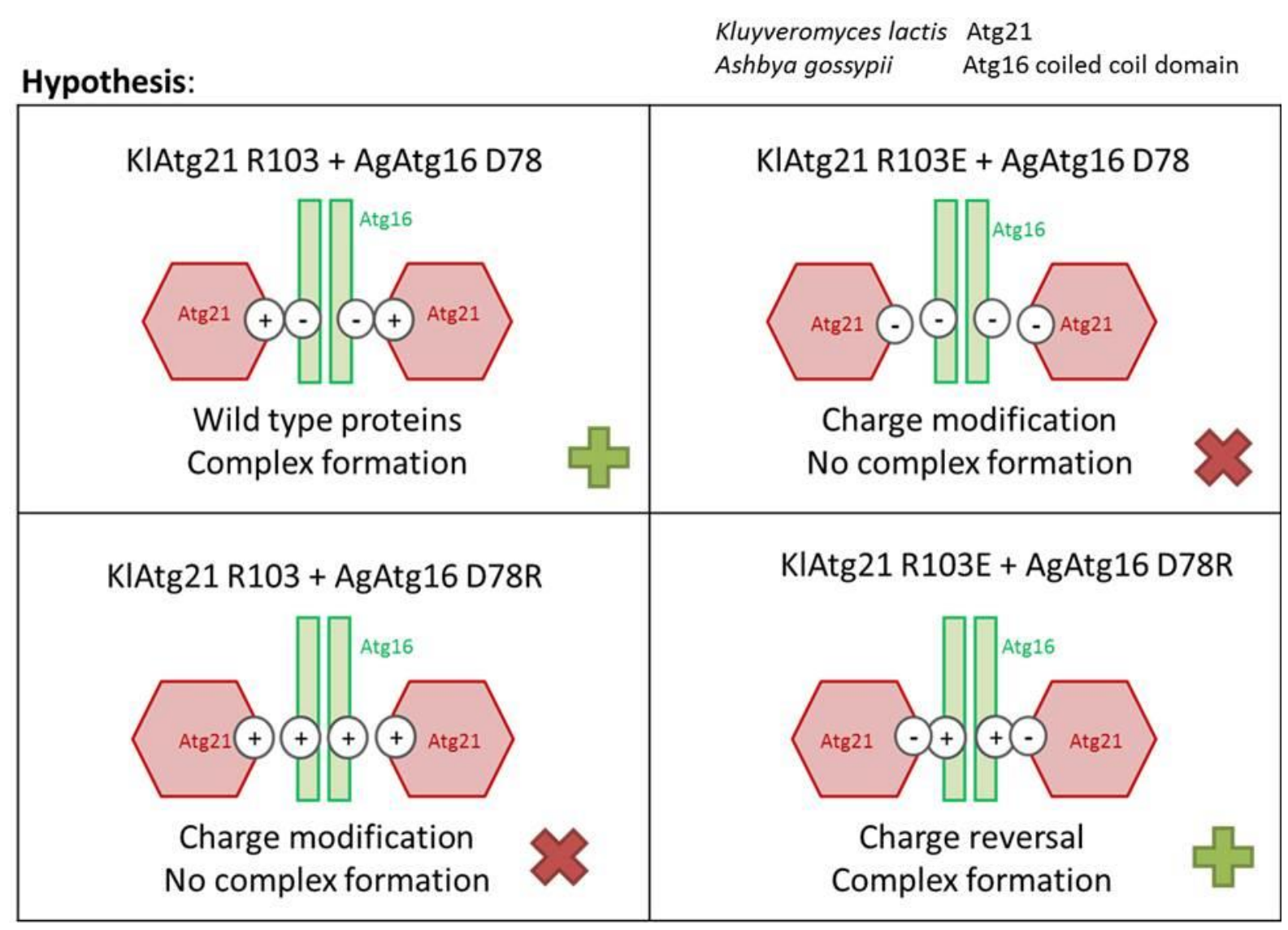

Figure 3.2.19.: Hypothesis for KlAtg21 and AgAtg16 complex formation. 


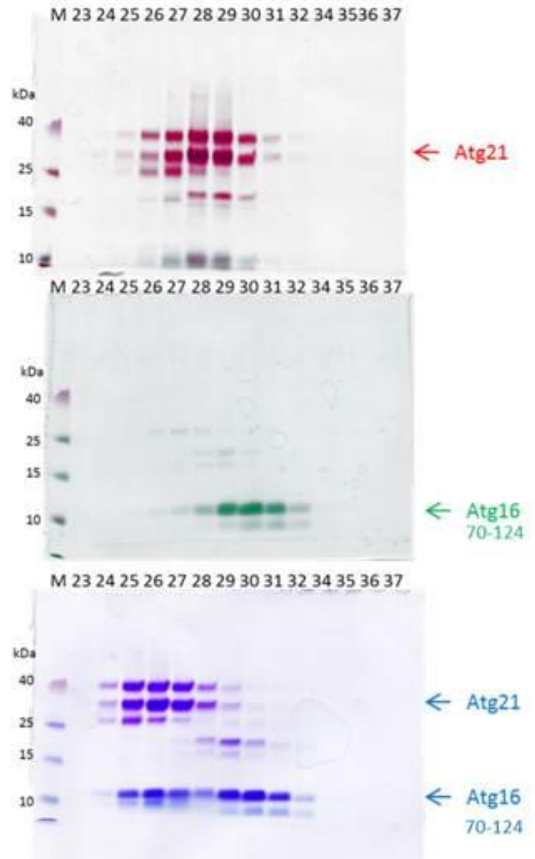

Figure 3.2.20.: Analytic gel filtration of KlAtg21 and AgAtg16 (70-124). Chromatograms of size exclusion chromatography using a Superdex 200 10/300 GL column. Red curve: KlAtg21, green curve: AgAtg16 (70-124), blue curve: KlAtg21 and AgAtg16 (70-124). Schägger gel electrophoresis of elution fractions are shown on the right side. $\mathrm{M}=$ Marker, Numbers refer to elution fractions. Bands were colored according to chromatogram color.

KIAtg21 fl R103E

AgAtg16 (70-124)

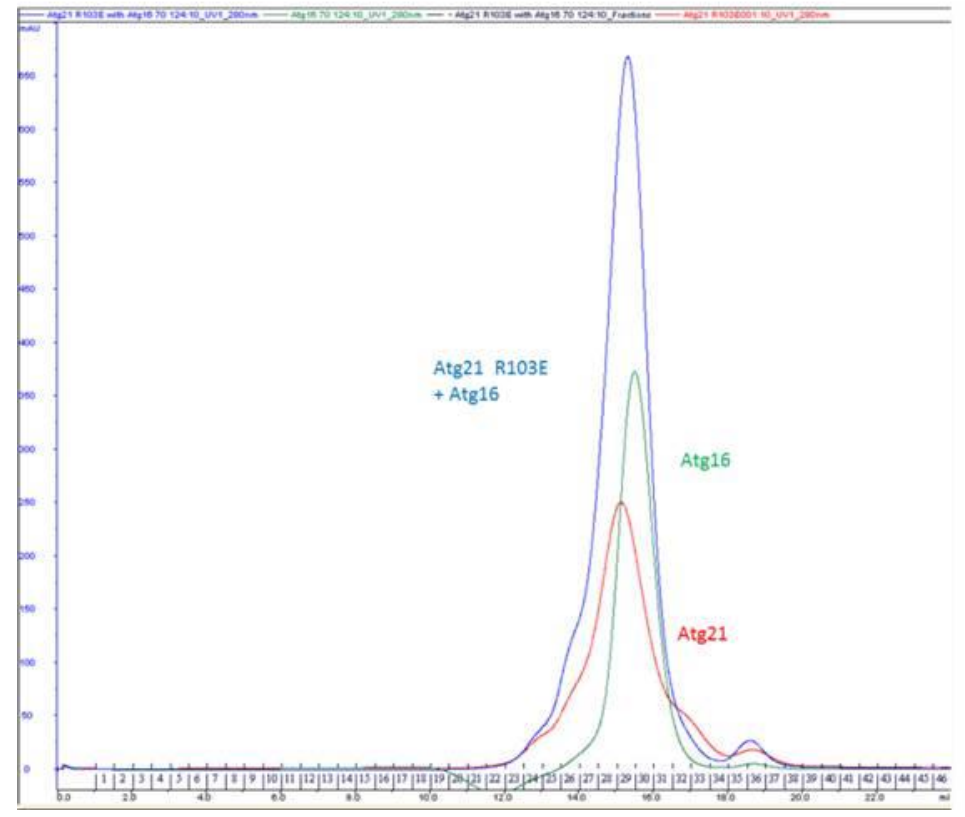

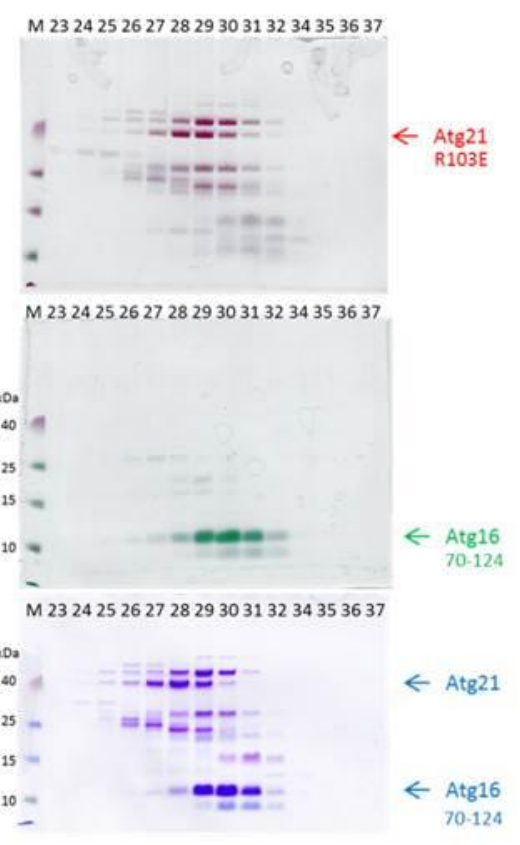


Figure 3.2.21.: Analytic gel filtration of KlAtg21 R103E and AgAtg16 (70-124). Chromatograms of size exclusion chromatography using a Superdex 200 10/300 GL column. Red curve: KlAtg21 R103E, green curve: AgAtg16 (70-124), blue curve: K1Atg21 R103 E and AgAtg16 (70-124). Schägger gel electrophoresis of elution fractions are shown on the right side. M=Marker, Numbers refer to elution fractions. Bands were colored according to chromatogram color.

\section{KIAtg21 fl \\ AgAtg16 (70-124) D78R}

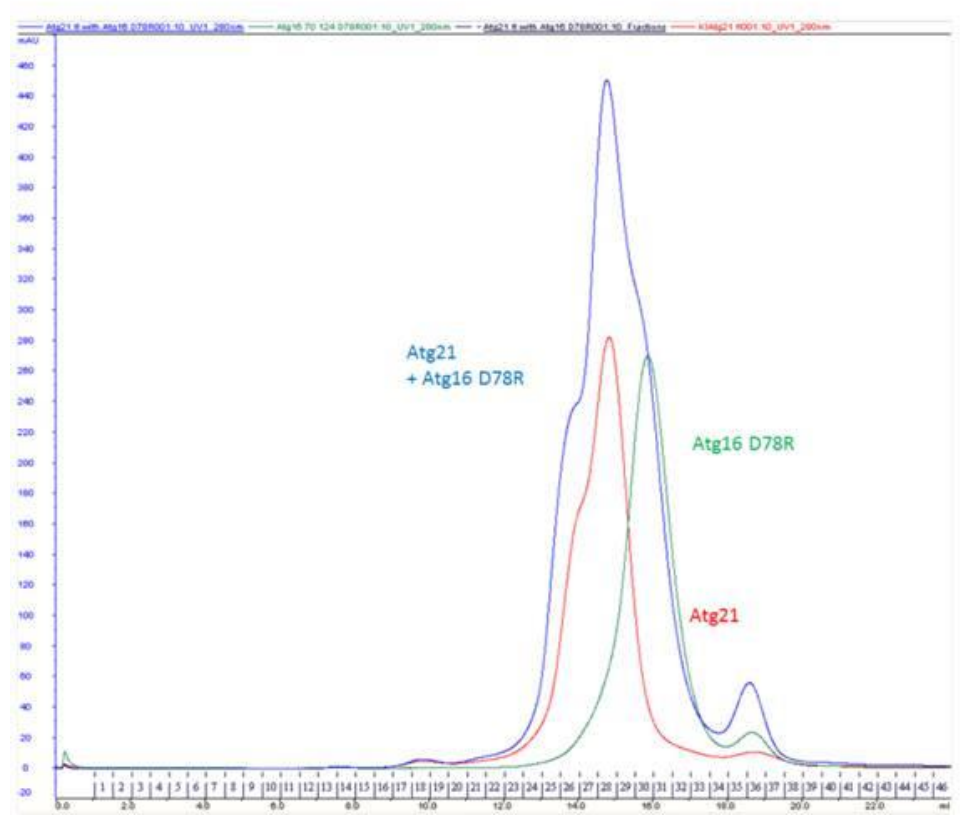

M 2324252627282930313234353637

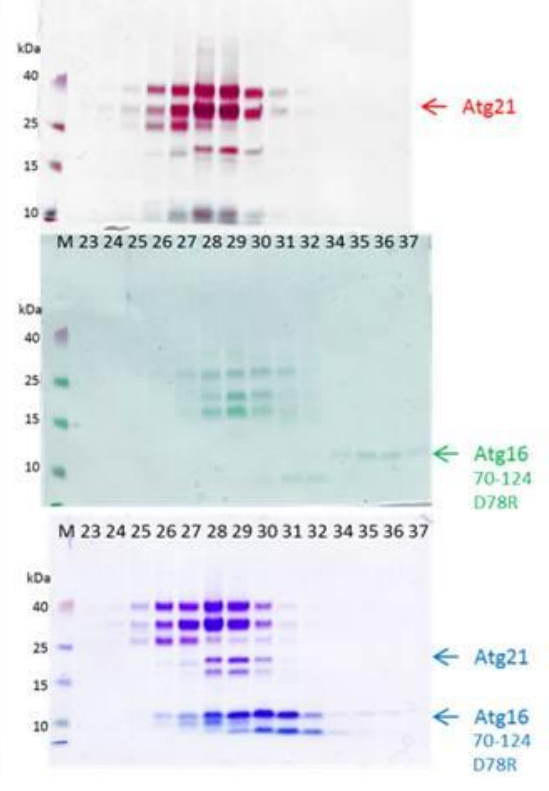

Figure 3.2.22.: Analytic gel filtration of KIAtg21 and AgAtg16 (70-124) D78R. Chromatograms of size exclusion chromatography using a Superdex 200 10/300 GL column. Red curve: KlAtg21, green curve: AgAtg16 (70-124) D78R, blue curve: KlAtg21 and AgAtg16 (70-124) D78R. Schägger gel electrophoresis of elution fractions are shown on the right side. M=Marker, Numbers refer to elution fractions. Bands were colored according to chromatogram color. 

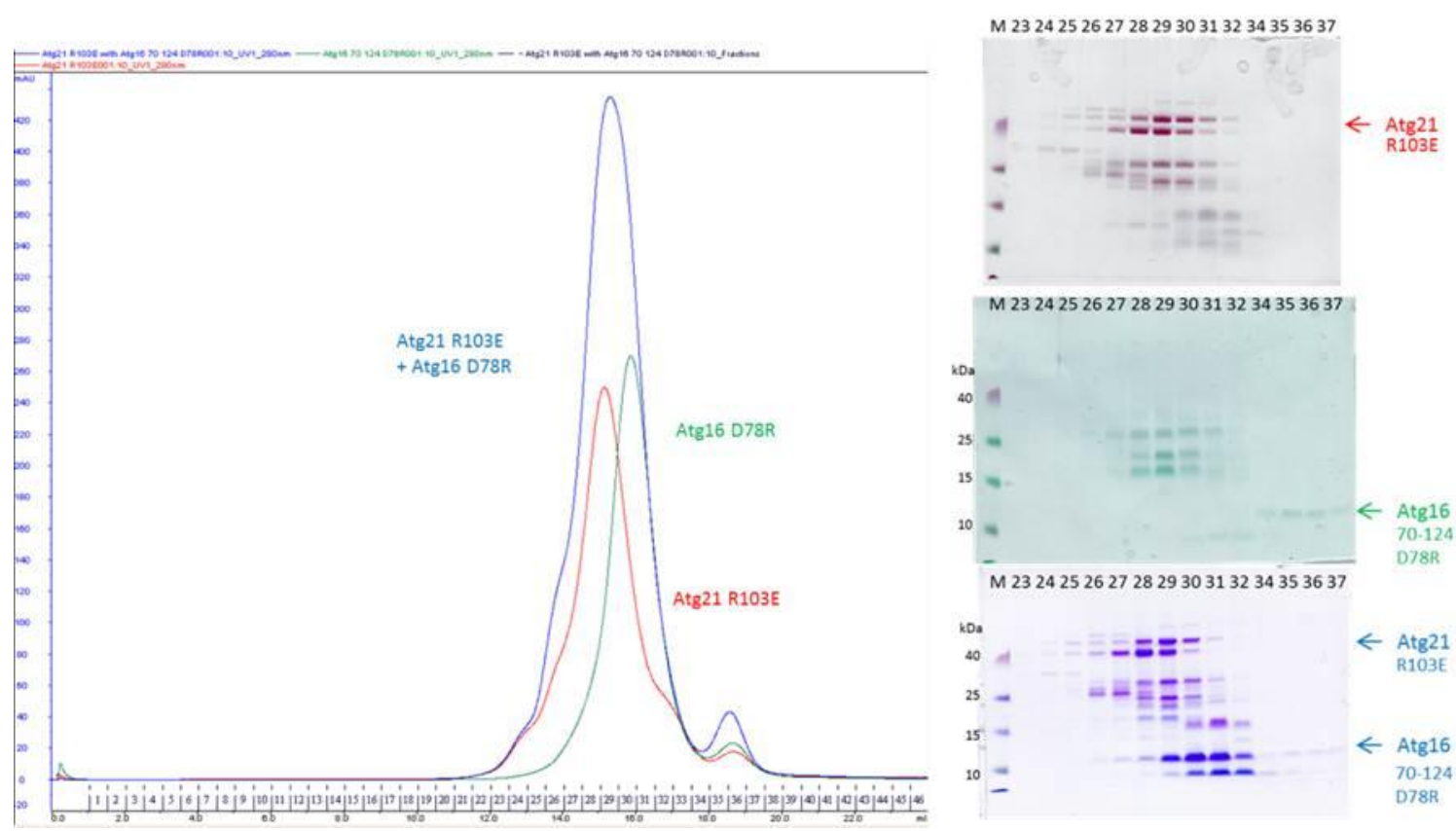

Figure 3.2.23.: Analytic gel filtration of KIAtg21 R103E and AgAtg16 (70-124) D78R. Chromatograms of size exclusion chromatography using a Superdex 200 10/300 GL column. Red curve: K1Atg21 R103E, green curve: AgAtg16 (70-124) D78R, blue curve: KlAtg21 R103E and AgAtg16 (70-124) D78R. Schägger gel electrophoresis of elution fractions are shown on the right side. $\mathrm{M}=$ Marker, Numbers refer to elution fractions. Bands were colored according to chromatogram color.

\subsubsection{Hypothetical model for the coordination of Atg21 and the Atg12 Atg5/Atg16 complex}

Solving the crystal structure of the KlAtg21-AgAtg16 complex reveals a new structural piece how the Atg12 Atg5/Atg16 complex is recruited to the membrane and thereby defines the Atg8 lipidation site. With superimposition of the new Atg21-Atg16 complex structure and the known crystal structures of ScAtg16 (PDB code: 3A7P) and human Atg12 Atg5 conjugate in complex with an N-terminal fragment of Atg16L1 and a fragment of Atg3 (PDB code: 4NAW) and ScAtg18 (PDB code: 3VWX) in complex with a fragment of PfAtg3 (PDB code: 4EOY), I propose a model of the Atg21 bound Atg12 Atg5/Atg16 complex and how it interacts with Atg8 (Fig. 3.2.24.). Atg16 binds the Atg12 Atg5 conjugate at the N-terminal part. Atg12 Atg5 conjugate functions as an E3-like enzyme for Atg8 lipidation. Atg12 recruits Atg3 by direct interaction and rearranges its catalytic site. The C-terminus of Atg8 is processed by the cysteine protease Atg4 to expose a glycine. Atg8 is then activated by E1-like activating enzyme Atg7. Direct binding at the top of the Atg21 propeller opposite to Atg16 
localizes Atg8 to a pool of phosphatidylethanolamine. Atg3 acts as an E2 enzyme for Atg8 and conjugates Atg8-PE.

A
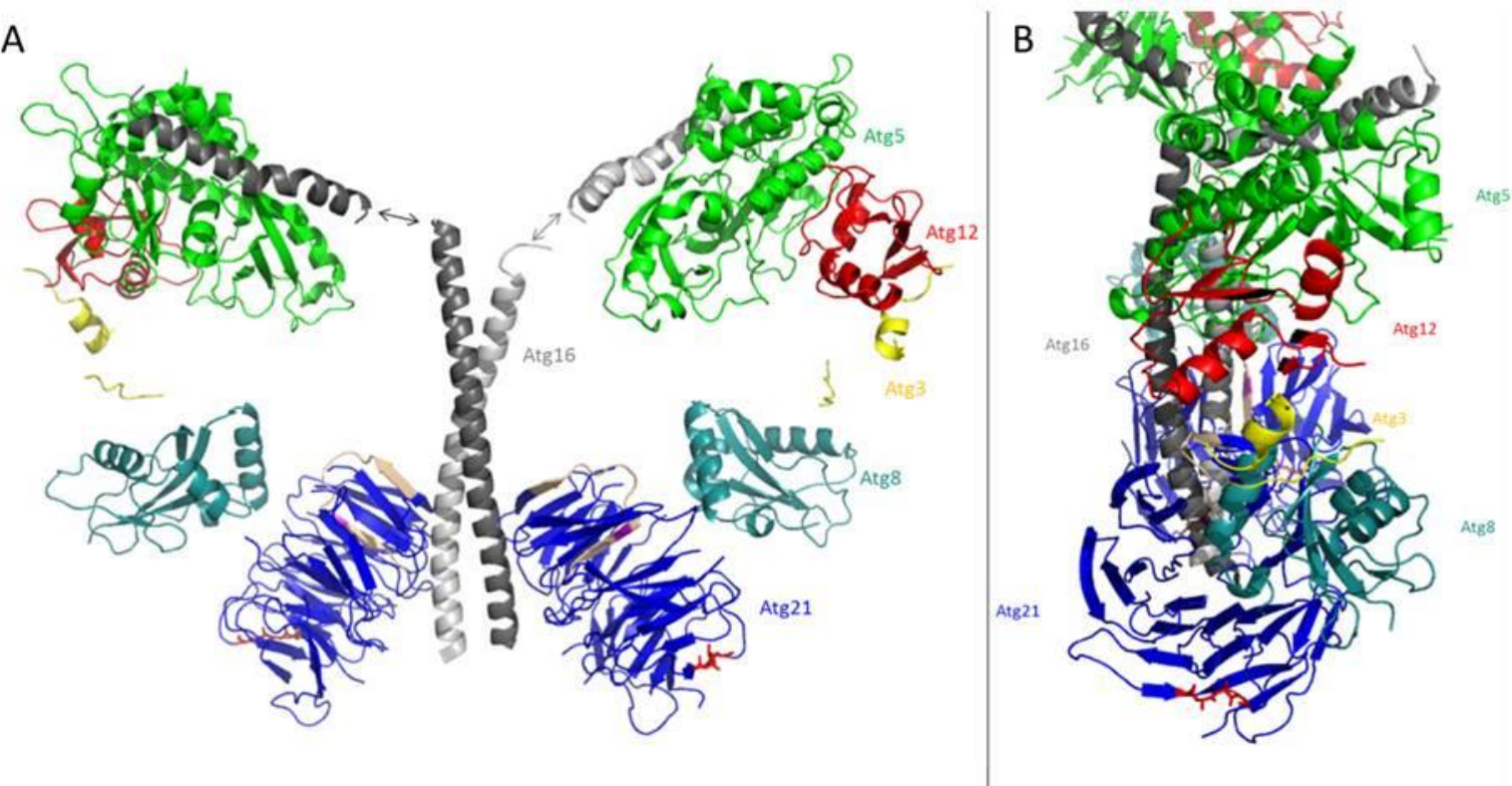

Figure 3.2.24.: Hypothetical model of Atg21 bound Atg12 Atg5/Atg16 complex interacting with Atg8 and Atg3. Cartoon representation of the complex. Model was made by superimposition and manual arrangement. KlAtg21 in dark blue, stick representation of the FRRG motif in red, Atg8 binding site in wheat, corresponding residue ScAtg21 D146 in pink. Atg16 (Superimposition of KlAtg21-AgAtg16/ C-terminal truncated coiled coil domain of ScAtg16, PDB code: 3A7P) in grey and human Atg12 Atg5/Atg16N with a fragment of Atg3 (PDB code: 4NAW), colored in red, green, grey and yellow respectively. Unknown linker between Atg16 coiled coil and Atg5-binding domain are marked with arrows. A fragment of PfAtg3 (PfAtg8-PfAtg3 complex, PDB code: 4EOY) in yellow defines the site of Atg8 interaction. PfAtg8 is replaced by ScAtg8 (ScAtg8ScAtg32, PDB code: 3VXW) in dark turquoise. A: Front view, B: Side view. 


\subsubsection{Structure of the AgAtg16 coiled coil domain}

\subsubsection{Crystallization of AgAtg16}

During screening of crystallization conditions for KlAtg21-AgAtg16 (40-124) by Dr. Karin Kühnel crystals appeared after 30 days in one condition of the Hampton SaltRX screen (Fig. 3.2.25.). Crystallization took place under in-situ proteolysis with 1:500 Clostripain (CL) at $20^{\circ} \mathrm{C}$ and could only be reproduced with this one purification batch.

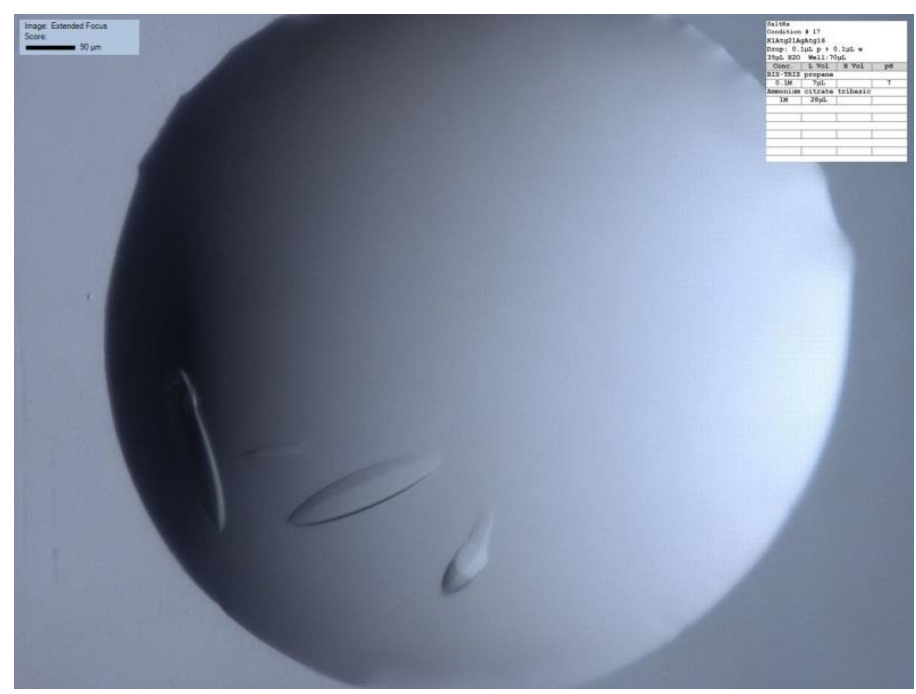

Figure 3.2.25.: Initial AgAtg16 (40-124) crystals. Crystals growing on a 96-well sitting drop plate. Crystals appeared after 30 days in SaltRX screen. Crystallization condition: 0.1 M Bis-Tris propane pH 7, 1 M Ammonium citrate tribasic. AgAtg16 (40-124) was mixed with 1:500 Clostripain.

These crystals appeared to have the same space group and very similar cell dimensions to SCOC-FEZ1 crystals observed from PEG 20000 conditions (Table 3.2.6.). Because of the very similar appearance and cell dimensions to SCOC-FEZ1 crystals, I took over all further experiments with these crystals.

Crystals were then identified as partially proteolytic digested AgAtg16 crystals by Schägger gel electrophoresis (Fig. 3.2.26.). The average purification batch of KlAtg21-AgAtg16(40124) contains a similar ratio of Atg21 to Atg16. In comparison, the purification batch which yielded AgAtg16 crystal did contain only a very small amount of Atg21. Clostripain digestion as used for in-situ crystallization reduced the quantity of full length Atg21 even more. 
Table 3.2.6.: Comparison of space group and cell dimensions of the AgAtg16 (40-124) and SCOCFEZ1 (grown in PEG 20.000 conditions) crystals

\begin{tabular}{|c|c|c|}
\hline & AgAtg16(40-124) crystal & SCOC-FEZ1 crystal \\
\hline Space group & $\mathrm{P}_{5} 22$ & $\mathrm{P}_{1} 22$ or $_{5} 22$ \\
\hline Cell dimensions & $106.15,106.15,148.94$ & $\sim 104, \sim 104, \sim 148$ \\
$\mathrm{a}, \mathrm{b}, \mathrm{c}(\AA)$ & $90.0, \quad 90.0,120.0$ & $90.0,90.0,120.0$ \\
$\alpha, \beta, \gamma\left(^{\circ}\right)$ & \multicolumn{2}{|c|}{$\left.{ }^{\circ}\right)$} \\
\hline
\end{tabular}

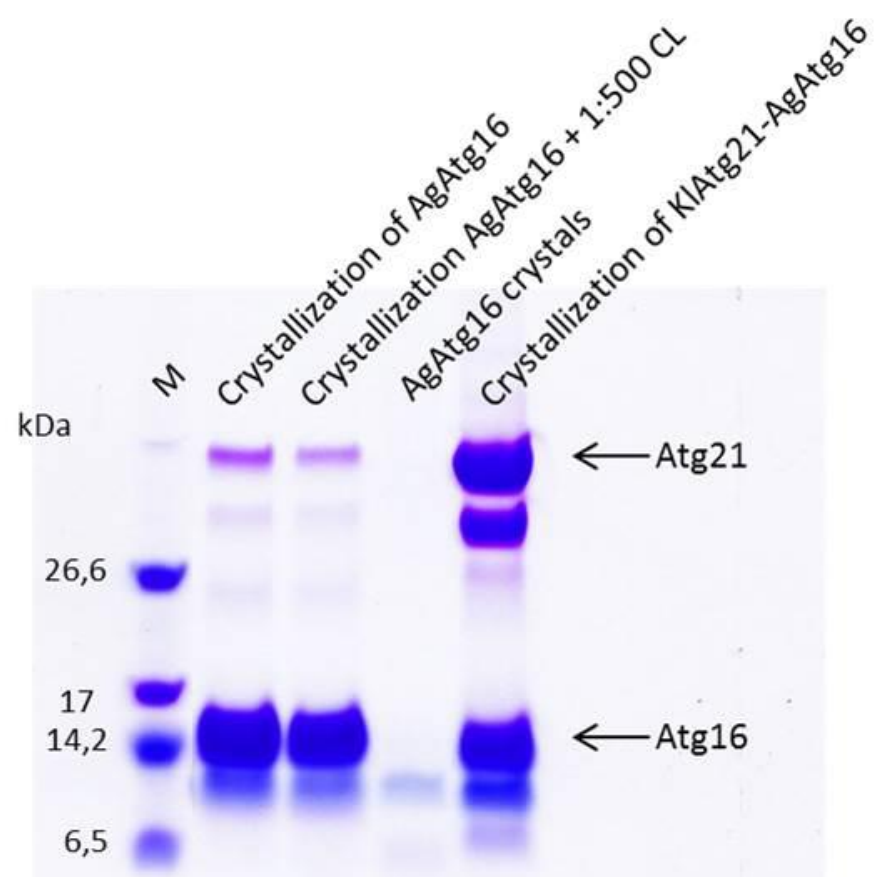

Figure 3.2.26.: Schägger gel electrophoresis of different KIAtg21-AgAtg16 (40-124) purification batches. M: Marker. 1. line shows the purification batch that gave the AgAtg16 crystals, 2. line same purification batch incubated with 1:500 Clostripain (CL), 3. lane: AgAtg16 crystals were fished and solubilized in gel filtration buffer, 4. lane: purification batch that was used for growing KlAtg21AgAtg16 crystals.

Crystallization conditions were optimized on 24-well hanging drop plates. Optimal crystallization conditions were found for drops composed of $3 \mu 16.8 \mathrm{mg} / \mathrm{ml} \mathrm{AgAtg} 16$ (40124) proteolysed with $1: 1000 \mathrm{CL}, 2 \mu \mathrm{l}$ crystallization solution made of $0.6 \mathrm{M}$ Bis-Tris propane $\mathrm{pH} 6.5$ and $0.6 \mathrm{M}$ ammonium citrate tribasic and $0.5 \mu 130 \%(\mathrm{w} / \mathrm{v})$ 1,5diaminopentane dihydrochloride. Huge hexagonal crystals started growing after 7 days (Fig. 3.2.27.). 

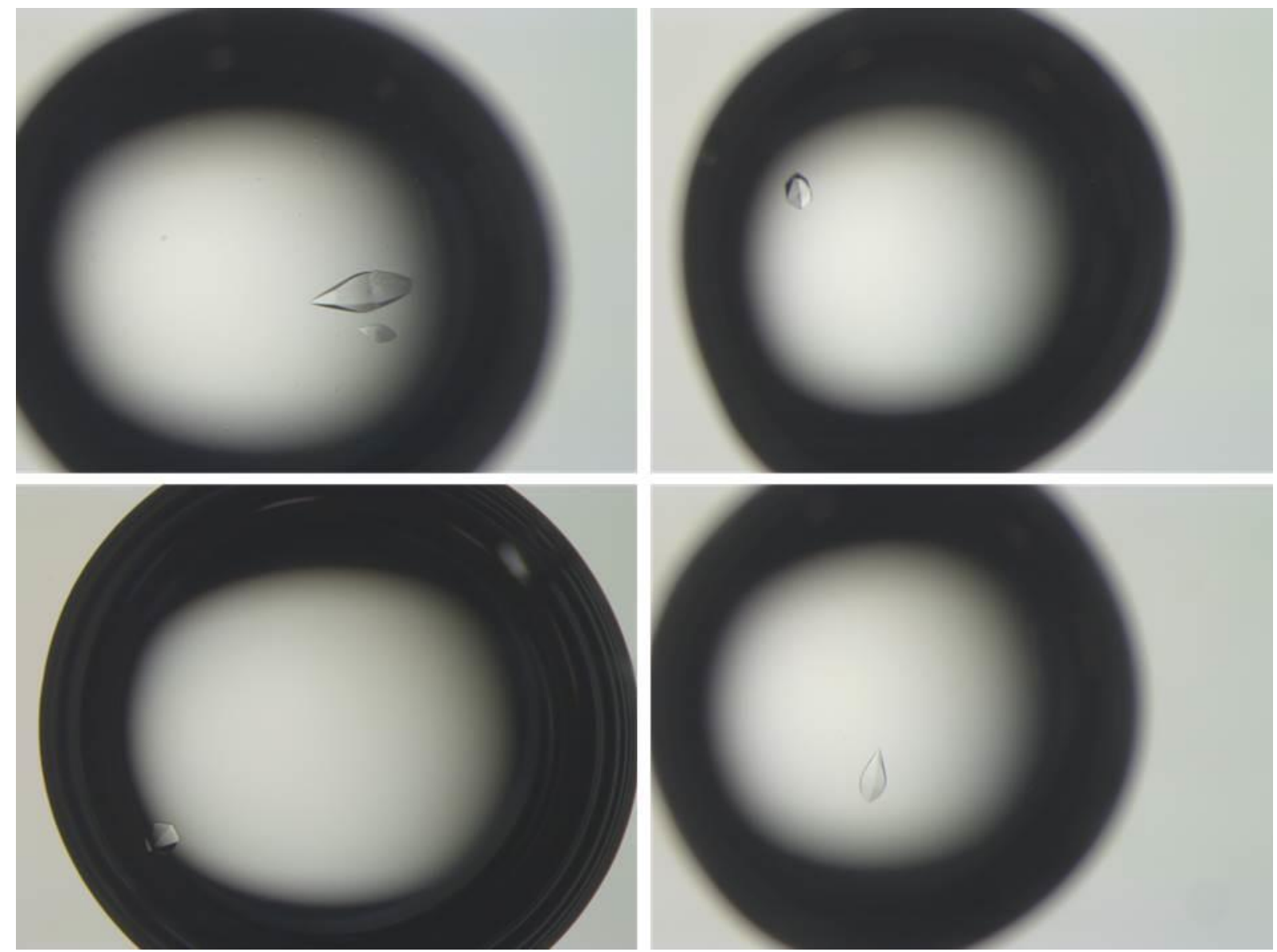

Figure 3.2.27.: Optimized AgAtg16 (40-124) crystals. Crystals appeared after 7 days in $0.1 \mathrm{M}$ BisTris propane $\mathrm{pH}$ 6.5, 0.6 M Ammonium citrate tribasic on 24-well Linbro plate.

\subsubsection{Mutagenesis of AgAtg16 for selenomethionine labeling}

Since the first low resolution datasets collected for AgAtg16 crystals were not sufficient for molecular replacement, structure determination was attempted by single-wavelength anomalous diffraction (SAD). Methionines in the protein can be labelled with Selenium by minimal expression with selenomethionine. AgAtg16(40-124) contains only two methionines at the $\mathrm{C}$ - and $\mathrm{N}$-terminus that might be flexible. To obtain a stronger anomalous signal a mutant was created that introduces an additional methionine. Isoleucine 81 was chosen because of its outer position in the heptad repeat, meaning that a mutation is very likely not to interfere with dimer formation. AgAtg16 (40-124) I81M was expressed and purified like the native protein (Fig. 3.2.28.). Functionality of AgAtg16 (40-124) I81M was tested by analyzing the complex formation with KlAtg21 by analytic gel filtration (Fig. 3.2.29.). Complex formation was observed but AgAtg16 (40-124) I81M did not crystallize. 


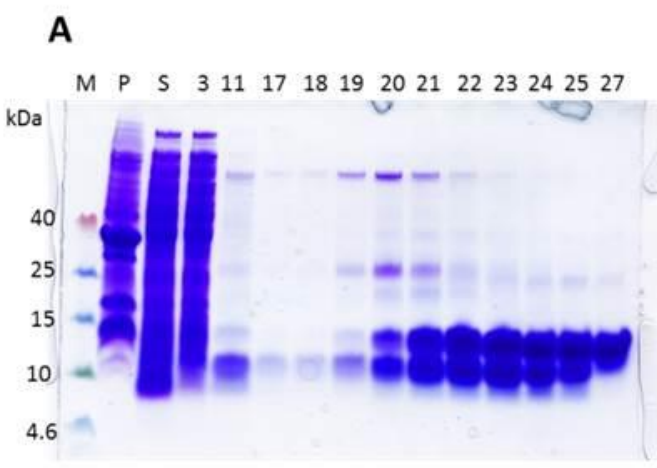

\section{B}

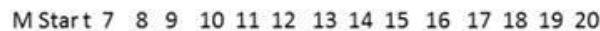

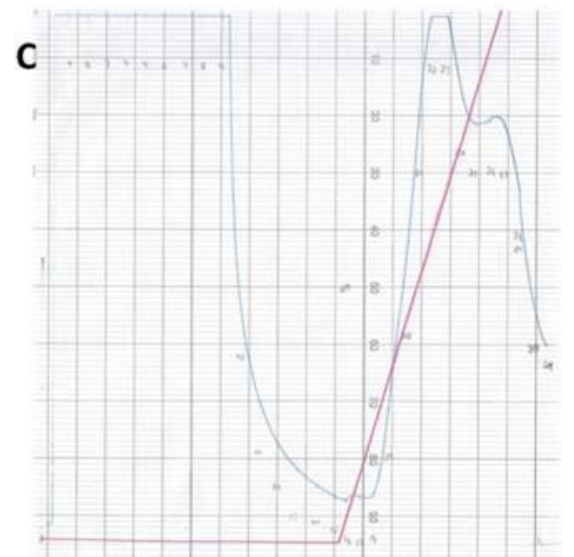

D
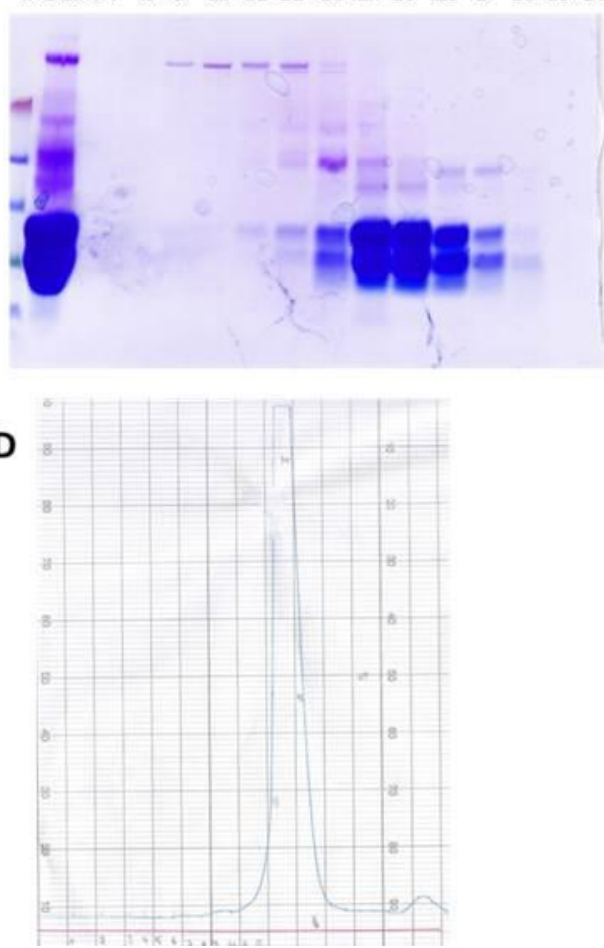

Figure 3.2.28.: Purification of AgAtg16 (40-124) I81M. A, C: HisTrap affinity chromatography. Schägger gel and corresponding chromatogram. B, D: Size exclusion chromatography using a HiLoad 16/60 Superdex 200 column. Schägger gel and corresponding chromatogram. M: Marker, Start: Input for SEC.

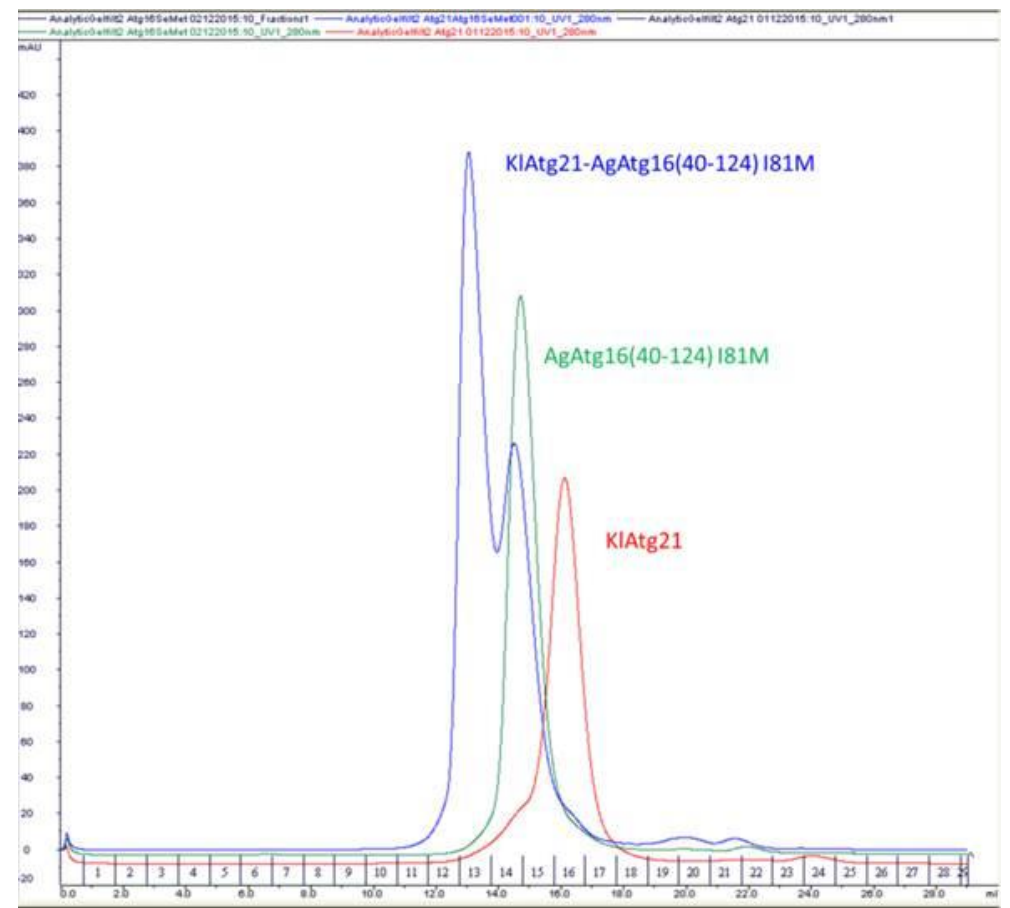

Figure 3.2.29.: Analytic gel filtration of KIAtg21 and AgAtg16 (40-124) I81M. Chromatograms of size exclusion chromatography using a Superdex 200 10/300 GL column. Red curve: KlAtg21, green curve: AgAtg16 (40-124) I81 M, blue curve: KlAtg21 and AgAtg16 (40-124) I81M. 


\subsubsection{Heavy metal soaking with magic triangle}

Another option to obtain phase information is the heavy-atom derivatization. With this technique anomalous scatterers like heavy atoms are incorporated into the protein crystal. Because heavy atom handling is risky the JBS Magic Triangle Phasing Kit was the first choice. The "Magic Triangle" 5-Amino-2,4,6-triiodoiso-phthalic acid (I3C) consists of three covalently bound iodine atoms forming an equilateral triangle with a side length of $6.0 \AA$ [10]. Compared to many heavy metal compounds IC3 has only low toxicity. IC3 was incorporated into AgAtg16(40-124) crystals by soaking and co-crystallization. For co-crystallization different concentrations of IC3 $(5,10$ and $15 \mathrm{mM})$ were added to the crystallization solution. Crystals grew in the same manner as native crystals. For soaking native crystals were transferred to a fresh drop of crystallization solution supplemented with 100,250 or $500 \mathrm{mM}$ IC3. Data for crystals with incorporated IC3 were collected at the same wavelength as an inhouse $\mathrm{Cu} \mathrm{K \alpha}$ beamline $(1.54 \AA)$ as suggested [10]. Crystal diffracted weaker than native crystals and the strength of the anomalous signal was not suitable for SAD phasing.

\subsubsection{X-ray data collection and processing for AgAtg16}

Data for structure determination of the AgAtg16 (40-124) were collected from a crystal growing in a 24-well hanging drop plate. Crystal was soaked in a solution of 1:1 crystallization solution and $30 \%$ ethylene glycol before flash cooling in liquid nitrogen. Data were collected at $100 \mathrm{~K}$ at beamline X10SA (Swiss Light Source, Paul Scherrer Institute, Villigen, Switzerland) (Fig. 3.2.30.). Four test shots were taken at the best position for indexing of the crystal Unit cell and collection strategies were determined with go.com (local software written by Dr. M. Wang).

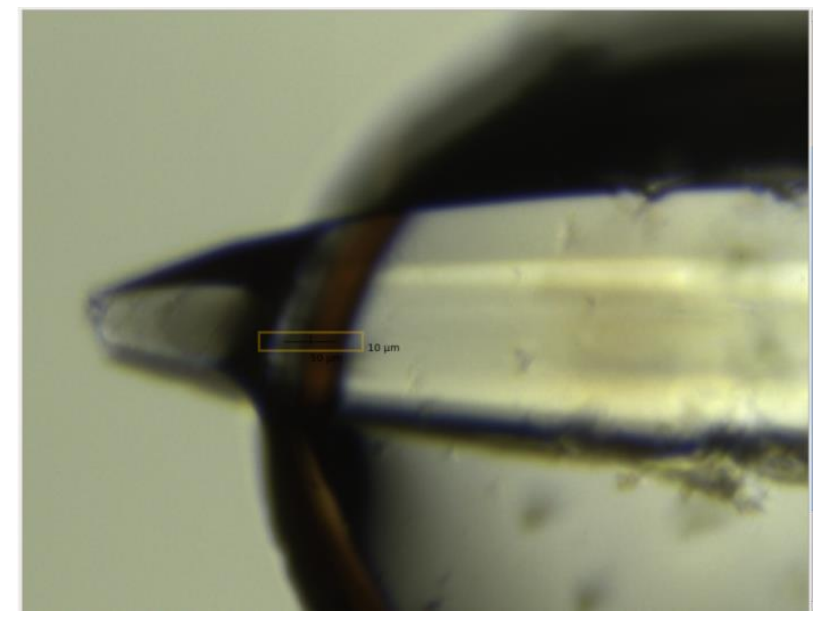

Figure 3.2.30.: AgAtg16 (40-124) crystal mounted at the Swiss Light Source. The yellow square has the size of $10 \times 50 \mu \mathrm{m}$. 
A native dataset of 360 degrees was collected at $0.979 \AA$ wavelength (for details see table 3.2.7.). Data were processed with input values specific for the PILATUS $6 \mathrm{M}$ detector using the XDS software package. XDS defined the space group as $\mathrm{P}_{5} 22$, which was confirmed by the absences of reflections along the screw axis $(0,0,6 \mathrm{n})$.

Table 3.2.7.: Data collection for AgAtg16(40-124) crystal

\begin{tabular}{|l|l|}
\hline Beamline & X10SA, Swiss Light Source (Switzerland) \\
\hline Detector & PILATUS 6M (Dectris) \\
\hline Detector distance & $300 \mathrm{~mm}$ \\
\hline$\varphi / \Delta \varphi$ & $0.25 / 360^{\circ}$ \\
\hline Exposure time & $0.25 \mathrm{sec}$ \\
\hline Beam intensity & 0.3 \\
\hline$\Lambda$ & $0.979 \AA$ \\
\hline Number of frames & 1440 \\
\hline
\end{tabular}

\subsubsection{Matthews coefficient for the AgAtg16(40-124) crystal}

The Matthews coefficient [84] was calculated using the CCP4 program suite to estimate the number of macromolecules in the asymmetric unit. This number is important to search for the correct number of molecules during structure determination. The molecular weight of a AgAtg16(40-124) dimer 19125 Da was used as input. With $74 \%$ probability the calculated Matthew coefficient (Table 3.2.8.) assumed two dimers per asymmetric unit with $41.77 \%$ solvent content. The demanding solvent content fits well with the relatively low diffraction of the crystals.

Table 3.2.8.: Matthews coefficient for AgAtg16(40-124) crystals. For estimated molecular weight of $19125 \mathrm{Da}$

Nmol/asym Matthews Coeff \%solvent $\quad \mathrm{P}(3.20) \quad \mathrm{P}($ tot $)$

\begin{tabular}{lcccc}
\hline 1 & 6.33 & 80.59 & 0.00 & 0.00 \\
2 & 3.17 & 61.18 & 0.35 & 0.25 \\
3 & 2.11 & 41.77 & 0.64 & 0.74 \\
4 & 1.58 & 22.36 & 0.00 & 0.00 \\
5 & 1.27 & 2.95 & 0.00 & 0.00
\end{tabular}

\subsubsection{Molecular replacement for AgAtg16}

Since selenomethionine labeled protein did not crystallize and soaking of the crystals in IC3 for phasing gave no significant anomalous signal, the structure was determined by molecular replacement. Phaser_MR in the CCP4 program suite was used to successfully search for two 
dimers with the structure of yeast Atg16 (PDB code 3A7O) as search model. ScAtg16 and AgAtg16 are highly conserved in the coiled coil domain (Fig. 3.2.31.). After one cycle of refinement with Refmac5 the initial model of AgAtg16 was build using arp_warp_classic.
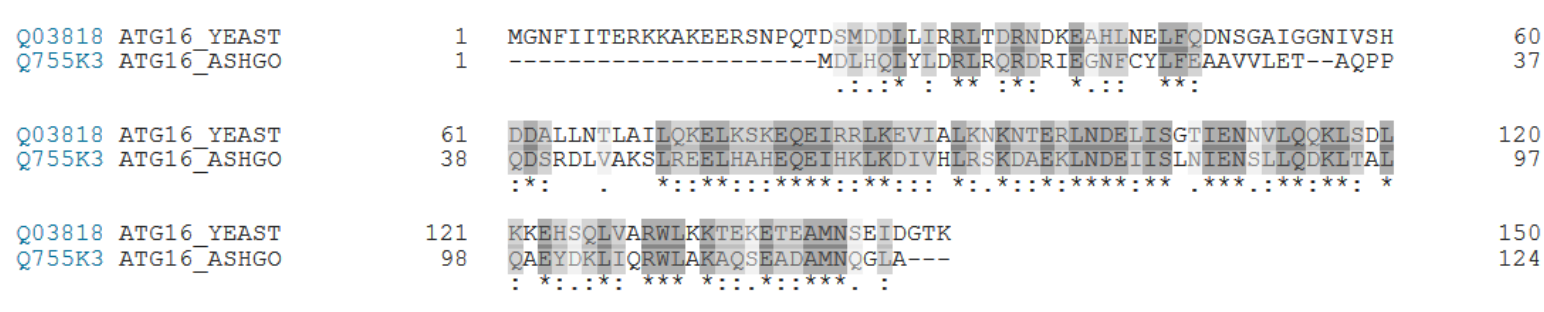

Figure 3.2.31.: Alignment of ScAtg16 and AgAtg16. Alignment was done using Clustal omega. Shading indicate level of conservation.

\subsubsection{Refinement and structure validation for AgAtg16}

The AgAtg16 structure was refined with Phenix and manual model building in Coot. The final round of refinement gave $\mathrm{R}_{\text {work }} / \mathrm{R}_{\text {free }}$ values of $0.27 / 0.33 \%$ (for complete data statistic for refinement see table 3.2.9.). The final model of AgAtg16 (40-124) contains four molecules in the asymmetric unit forming two coiled coil dimers with chains $A B$ and $C D$. The model includes residues 43-108 for the dimer with chains A and B and 52-108 for the dimer with chains $\mathrm{C}$ and $\mathrm{D}$. The structure was validated using Phenix polygon plot that compares the refinement statistics to structures in the PDB with a similar resolution cut-off (Fig. 3.2.32. A). All values are within the permitted range. The Ramachandran plot was calculated by Phenix and used to analyze the geometry of the model (Fig. 3.2.32. B). $96 \%$ of the residues are within the preferred regions, $3.8 \%$ in the allowed regions and $0.4 \%$ outliers were found. 
Table 3.2.9.: Data collection and refinement statistics of AgAtg16 (40-124). Statistics for the highest-resolution shell are shown in parentheses. Ramachandran statistics were calculated with Molprobity.

\begin{tabular}{|c|c|}
\hline $\begin{array}{l}\text { Data collection } \\
\text { Space group }\end{array}$ & $\mathrm{P}_{5} 22$ \\
\hline $\begin{array}{l}\text { Cell dimensions } \\
\text { a, b, c }(\AA) \\
\alpha, \beta, \gamma\left(\left(^{\circ}\right)\right.\end{array}$ & $\begin{array}{c}106.15,106.15,148.94 \\
90.0, \quad 90.0,120.0\end{array}$ \\
\hline $\begin{array}{l}\text { Resolution range }(\AA) \\
\text { Total reflections } \\
\text { Unique reflections } \\
\text { Multiplicity } \\
\text { Completeness }(\%) \\
\text { Mean } \mathrm{I} \sigma \\
\text { Wilson B factor }\left(\AA^{2}\right) \\
\mathrm{R}_{\text {meas }}(\%) \\
\mathrm{CC}_{1 / 2}\end{array}$ & $\begin{array}{l}43.92-3.4(3.52-3.4) \\
268583(27945) \\
7284(714) \\
36.8 \\
99.8(99.9) \\
34.35(4.34) \\
113.0(96.56) \\
9.2(112.7) \\
100(93.9)\end{array}$ \\
\hline $\begin{array}{l}\text { Refinement } \\
\mathrm{R}_{\text {work }} \\
\mathrm{R}_{\text {free }}\end{array}$ & $\begin{array}{l}0.270(0.371) \\
0.327(0.506)\end{array}$ \\
\hline $\begin{array}{l}\text { Number of non-hydrogen atoms: } \\
\text { Molecules/AU } \\
\text { Number of protein residues included in model: }\end{array}$ & $\begin{array}{l}1966 \\
4 \\
246 \\
\text { A: } 43-108 \\
\text { B: } 43-108 \\
\text { C: } 52-108 \\
\text { D: } 52-108\end{array}$ \\
\hline $\begin{array}{l}\text { B-factors }\left(\AA^{2}\right) \\
\text { Average } \\
\text { Structure validation } \\
\text { Ramachandran favored (\%) } \\
\text { Ramachandran allowed (\%) } \\
\text { Ramachandran outliers (\%) } \\
\text { Clashscore }\end{array}$ & $\begin{array}{l}104.3 \\
96 \\
3.8 \\
0.4 \\
2.0\end{array}$ \\
\hline $\begin{array}{l}\text { RMSD deviations } \\
\text { Bond lengths }(\AA) \\
\text { Bond angles }\left({ }^{\circ}\right)\end{array}$ & $\begin{array}{l}0.003 \\
0.61\end{array}$ \\
\hline
\end{tabular}


A

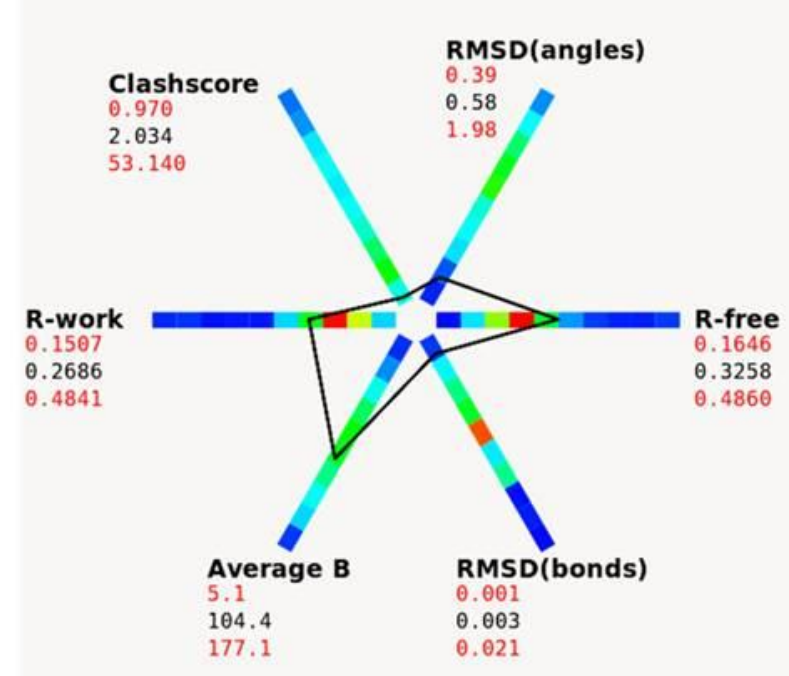

B

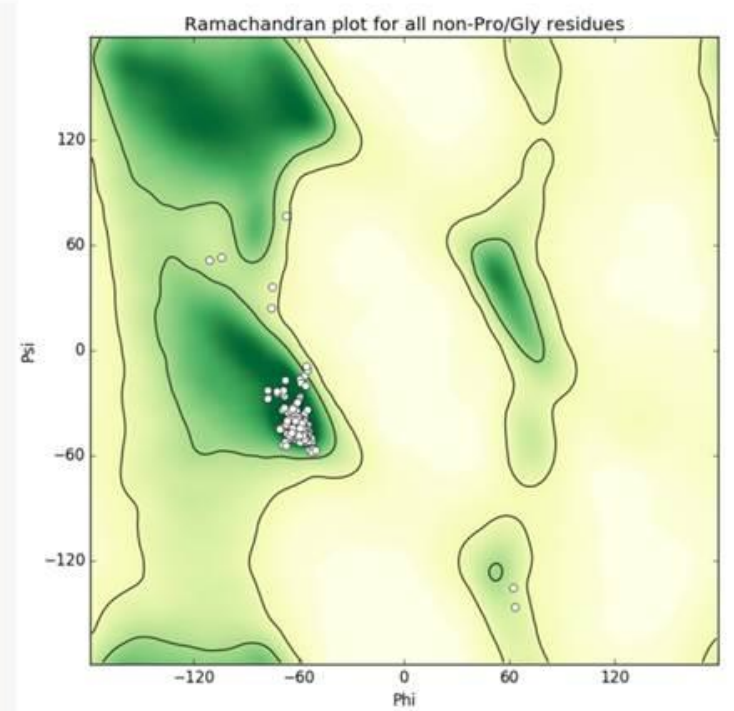

Figure 3.2.32.: Refinement statistic plots for AgAtg16 (40-124). A: Polyglon plot. Structure statistics (black numbers) are compared to PDB entries with similar resolution (red numbers). B: Ramachandran plot of all non Pro/Gly residues. Plots were made by Phenix refine.

\subsubsection{Structure analysis for AgAtg16}

X-Ray structure of AgAtg16(40-124) reveals that AgAtg16 is a parallel oriented, left-handed coiled coil dimer. The asymmetric unit contains four AgAtg16 molecules A, B, C and D that form two homodimers $\mathrm{AB}$ and $\mathrm{CD}$ (Fig. 3.2.33.). 


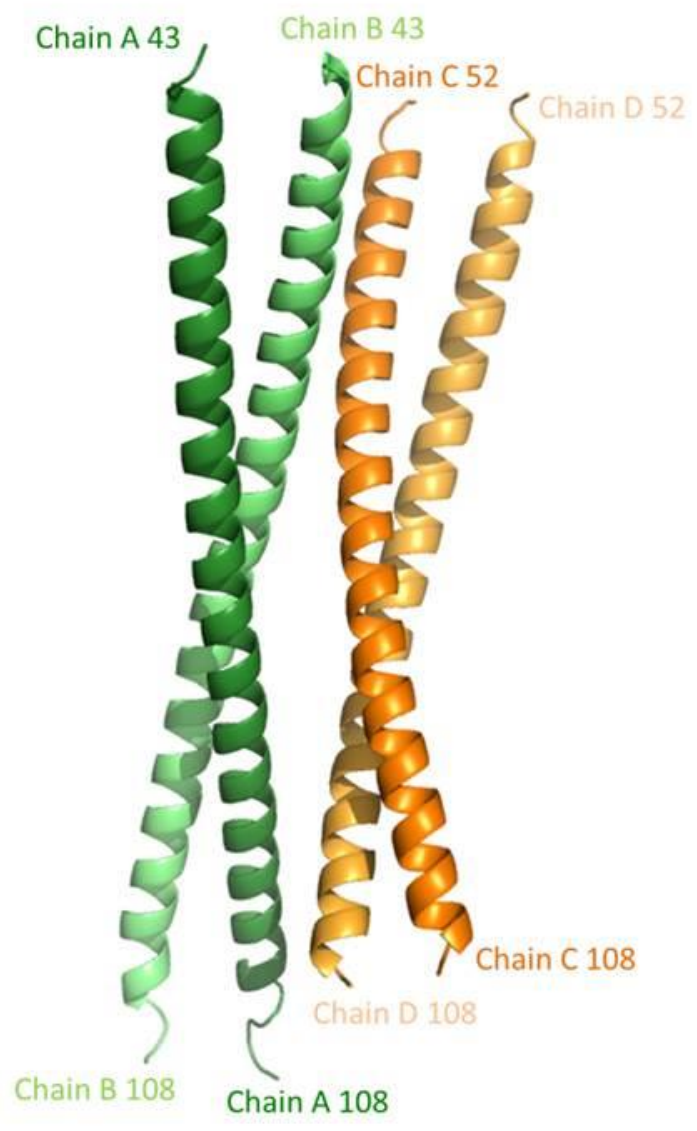

Figure 3.2.33.: Four AgAtg16 molecules in the asymmetric unit. Two AgAtg16 molecules form a dimer respectively. Cartoon representation. Chains are colored as A dark green, B light green, C orange, D light orange.

Packing of the crystal is not very loose and does not explain the low diffraction limit (Fig. 3.2.34.). The two dimers do not overlap perfectly with a RMSD of $1.536 \AA$ for the C $\alpha$ atoms. Dimer AB comprises residues 43-108 that form nine heptad repeats and has a length of about $100 \AA$. The N-terminus of dimer CD is disordered, therefore dimer CD compromises residues 52 to 108 with a length of about $85 \AA$ and eight heptad repeats. The coiled coil radius of 5.18, calculated with TWISTER [130], is similar to coiled coil radius of 4.85 that was measured for the leucine zipper GCN4 dimer (PDB code: 2TZA). 


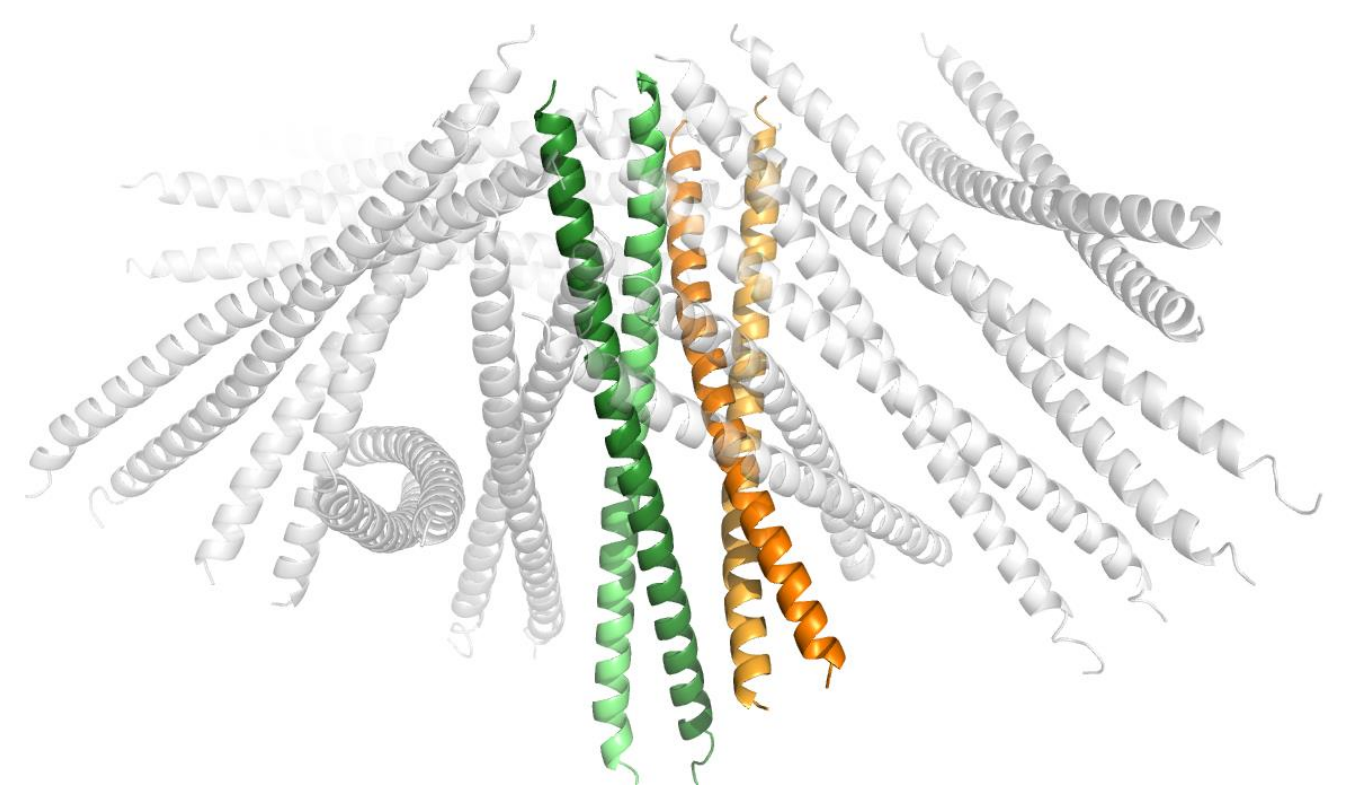

Figure 3.2.34.: Crystal packing of the AgAtg16 crystal. Four molecules forming two AgAtg16 dimers in the asymmetric unit are shown in color. Crystallographic symmetry-related molecules are shown in grey.

Calculating the electrostatic potential shows mainly positive charged amino acids at the dimer interface and negatively charged amino acids on the AgAtg16 dimer surface (Fig. 3.2.35.). For AgAtg16 ccd the coiled coil heptad repeat pattern "abcdefg" is mainly engaged of polar residues at position a and hydrophobic residues at position d (Fig. 3.2.36.).

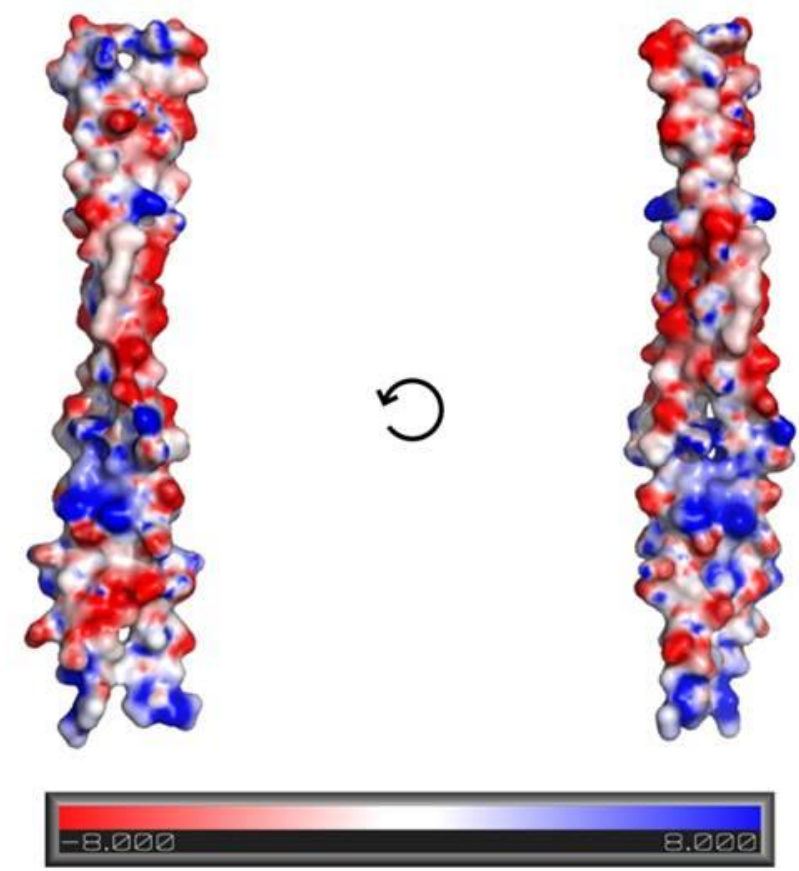

Figure 3.2.35.: Electrostatic potential surface for AgAtg16 dimer AB. Electrostatic potential were generated using the adaptive Poisson-Boltzmann Solver (APBS). 


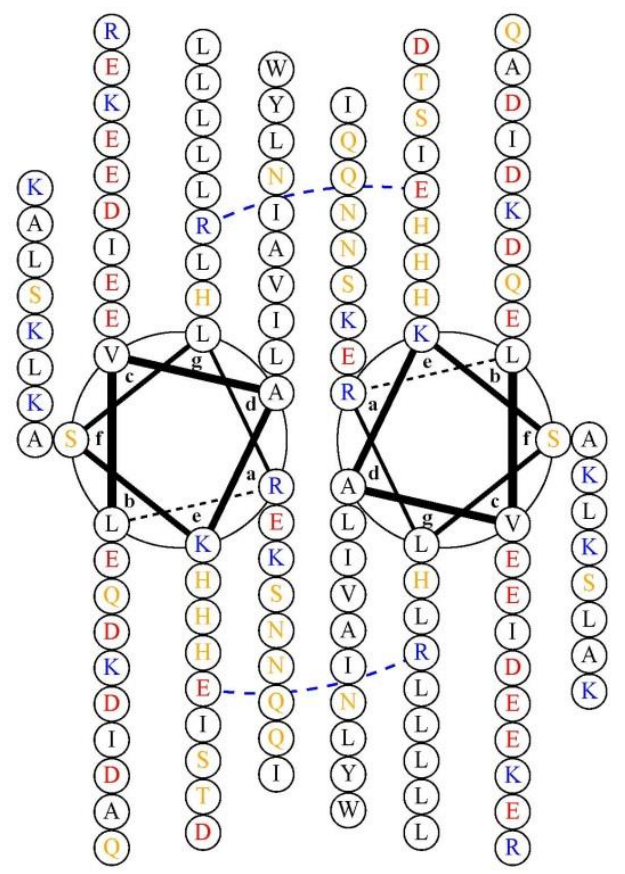

Figure 3.2.36.: Helical wheel projection of the residues in the AgAtg16 dimer AB. Black: hydrophobic residues, Yellow: polar residues, Red: acidic residues, Blue: basic residues. Dashed lines show potential salt bridges. Helical wheel was made using DrawCoil 1.0 [46]. 


\subsection{Characterization of the SCOC-FEZ1 complex}

Because SCOC-FEZ1 complex plays a role in the regulation of autophagy the goal of our laboratory was to characterize and determine the structure of the SCOC-FEZ1 complex. The coiled coil domain of SCOC (78-159) was already solved and published by Dr. Caroline Behrens from our laboratory [11]. FEZ1 is a mainly natively unfolded protein and therefore a difficult target for crystallography. Furthermore, recombinant expressed full length FEZ1 was not soluble under native conditions. Studies from Dr. Caroline Behrens on the SCOC-FEZ1 complex showed that SCOC dimerization and SCOC residue R117 is vital for complex formation. She found that FEZ1 residues M227 to L290 provide the minimal coiled coil domain to form a complex with SCOC (78-159) [12].

\subsubsection{Expression and purification of SCOC-FEZ1 complexes}

Strep tagged SCOC ccd and His tagged FEZ1 ccd were coexpressed. The purification protocol of Dr. C. Behrens used a StrepTrap column and then size exclusion chromatography. The size exclusion chromatogram shows that there is SCOC that did not form a complex in the purified protein solution (Fig. 3.3.1 A, C). Since crystallization attempts resulted in SCOC crystals only, the purification of the complex was optimized. An additional Ni-Sepharose purification step before the StrepTrap column was introduced. With two steps of affinity chromatography interaction partners that did not form a complex are removed. Size exclusion chromatography showed only one peak and both proteins were evenly distributed on a coomassie stained Schägger gel (Fig. 3.3.1 B, D). 

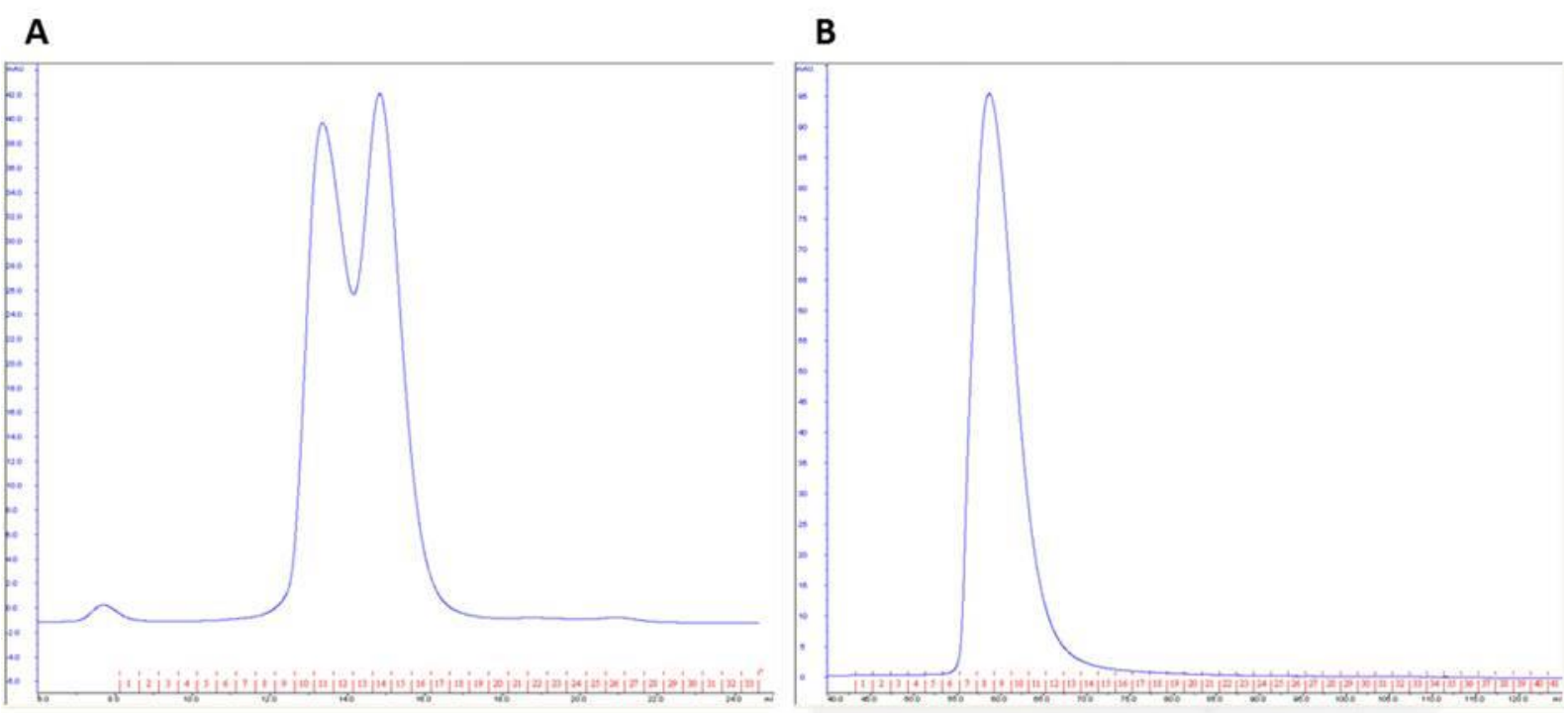

C

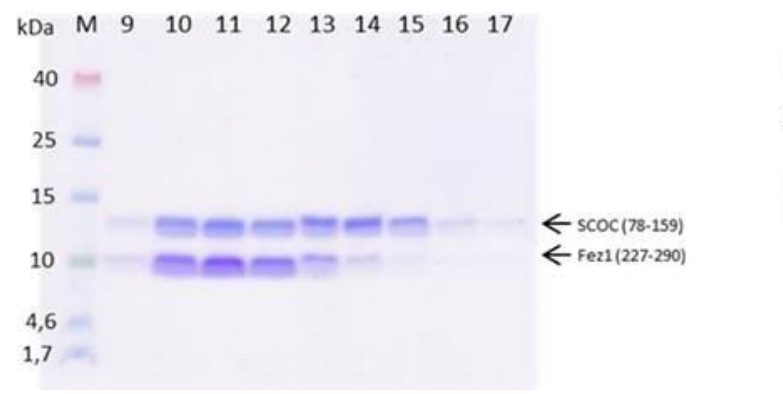

D

$\begin{array}{llllllll}M & 7 & 8 & 9 & 10 & 11 & 12 & 13\end{array}$

kDa

26,6

17

$\leftarrow \operatorname{scoc}(78-159)$

$\leftarrow \operatorname{Fez1}(227-290)$

Figure 3.3.1.: Purification of SCOC (78-159) FEZ1 (227-290) complexes. Elution profiles of SCOC (78-159) FEZ1 (227-290) size exclusion chromatography. A: Size exclusion chromatography by Superdex 200 10/300 GL after step StrepTrap affinity chromatography. B: Size exclusion chromatography by HiLoad 16/60 Superdex 75 prep grade after Ni-Sepharose purification followed by StrepTrap affinity chromatography. C: Schägger gel analysis of selected fractions from A. D: Schägger gel analysis of selected fractions from B. M: Marker. Numbers correspond to elution fractions.

The minimal complex was not very stable and in crystallization trials only SCOC crystallized (see 3.3.3.1). Since SCOC has a mainly negative surface charge and the coiled coil domain of FEZ1 has a number of negative charged residues, a longer FEZ1 (225-295) construct was chosen for SCOC (78-159) complex formation. The FEZ1 (225-295) construct contains seven more mainly positively charged residues. In contrast to FEZ1 (227-290) with a net charge of -5, FEZ1 (225-295) has a net charge of -1/0. Indeed, the complex of SOC (78-159)-FEZ1 (225-295) was much more stable and therefore used for all further experiments. The optimized purification was up scaled and done at $4{ }^{\circ} \mathrm{C}$ to avoid protein degradation (Fig. 3.3.2.). Protein complex was concentrated to $10-15 \mathrm{mg} / \mathrm{ml}$ and used for further experiments. 
A

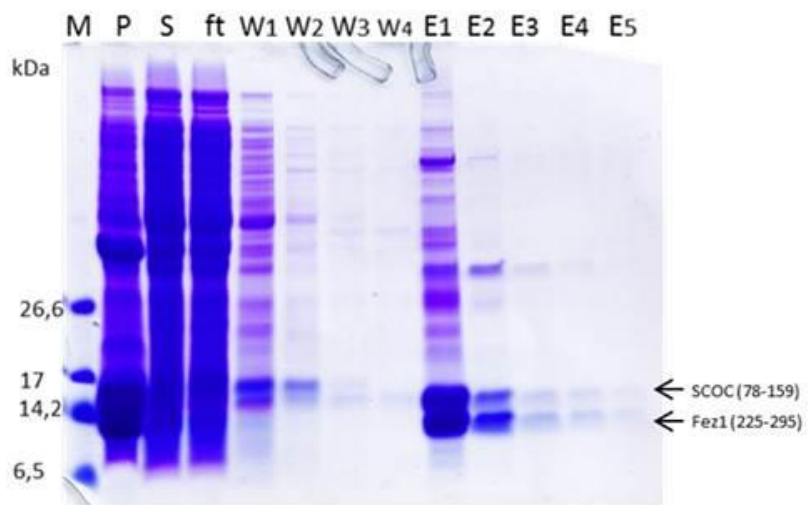

B kDa

M Start $2 \quad 812131415161718$
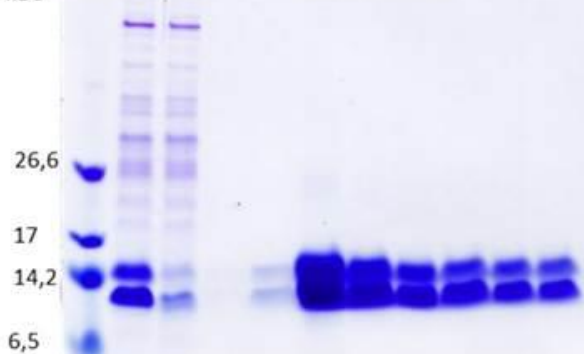

D

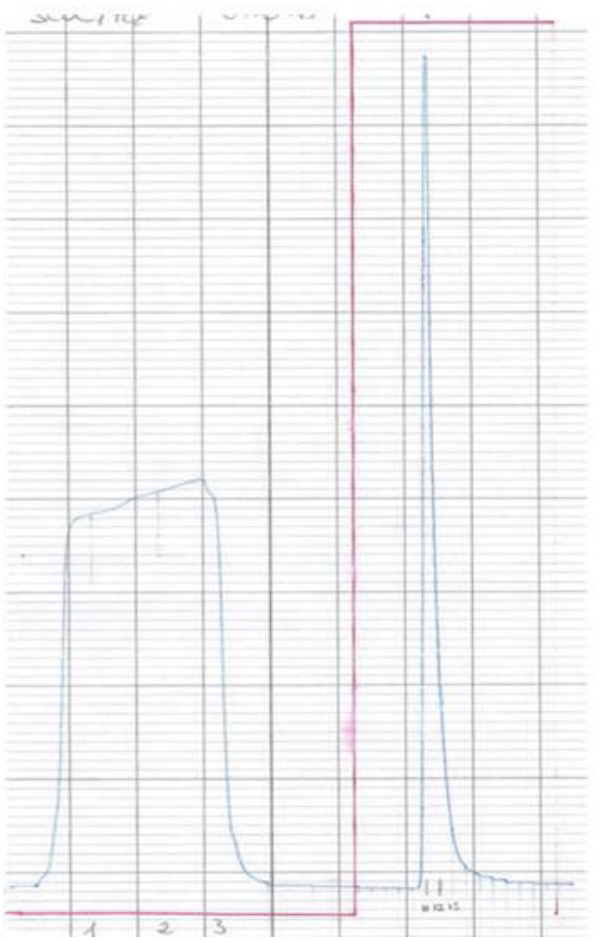

$E$

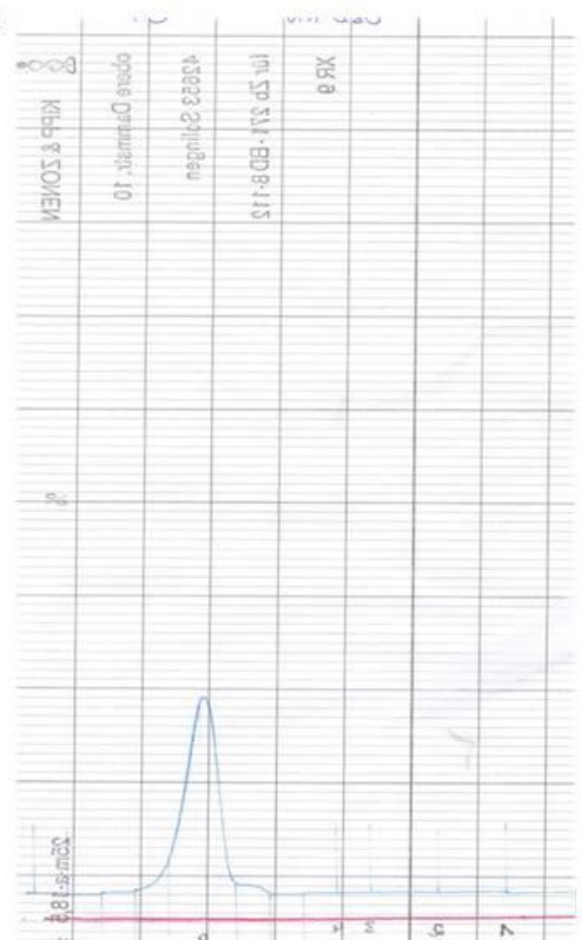

Figure 3.3.2.: Optimized purification of the SCOC (78-159)-FEZ1 (225-295) complex. A: Schägger gel analysis of Ni-Sepharose purification. B: Schägger gel analysis of selected fractions from StrepTrap chromatography. C: Schägger gel analysis of selected fractions from size exclusion chromatogram by HiLoad 16/60 Superdex 75 prep grade. D: Elution profile from StrepTrap chromatography. E: Elution profile from size exclusion chromatography. M: Marker, P: Pellet, S: Supernatant, ft: flow through, W1-4: Wash fractions, E1-4: Elution fractions, Start: Material loaded on column. Numbers correspond to elution fraction. 
Since coiled coil proteins are difficult targets for molecular replacement and only the SCOC structure is known, structure determination was attempted by SAD phasing using selenomethionine labeled protein. FEZ1 contains only two methionines at the $\mathrm{C}$ - and $\mathrm{N}$ terminus that might be flexible due to their location, which would give only a weak or no anomalous signal. To achieve a stronger anomalous signal two mutants were created with an additional methionine site, FEZ1 (225-295) L251M and L273M. FEZ1 mutants were coexpressed with SCOC (78-159) L105M that was used for solving the SCOC ccd structure by SAD phasing. Selenomethionine labeled protein complexes were purified in the same manner as native protein but included $2 \mathrm{mM} \beta$-Mercaptoethanol in all buffers.

\subsubsection{Biochemical characterization of the SCOC (78-159)-FEZ1 (225-295) complex}

3.3.2.1. Analysis of secondary structure and stability of the SCOC-FEZ1 complex by CD spectroscopy

The SCOC-FEZ1 complex was analyzed by CD spectroscopy. The CD spectrum was measured from 180 to $260 \mathrm{~nm}$ at $20{ }^{\circ} \mathrm{C}$. The $\mathrm{CD}$ spectrum shows a maximum at $195 \mathrm{~nm}$ and two minima around 208 and $222 \mathrm{~nm}$ (Fig. 3.3.3. A), giving a typical curve for $\alpha$-helical secondary structure as expected for coiled coil domain proteins. This confirms folding of the protein complex. A melting curve was recorded at $222 \mathrm{~nm}$ from 20 to $90{ }^{\circ} \mathrm{C}$, the complex is stable with a melting temperature of $48^{\circ} \mathrm{C}$ (Fig. 3.3.3. B).
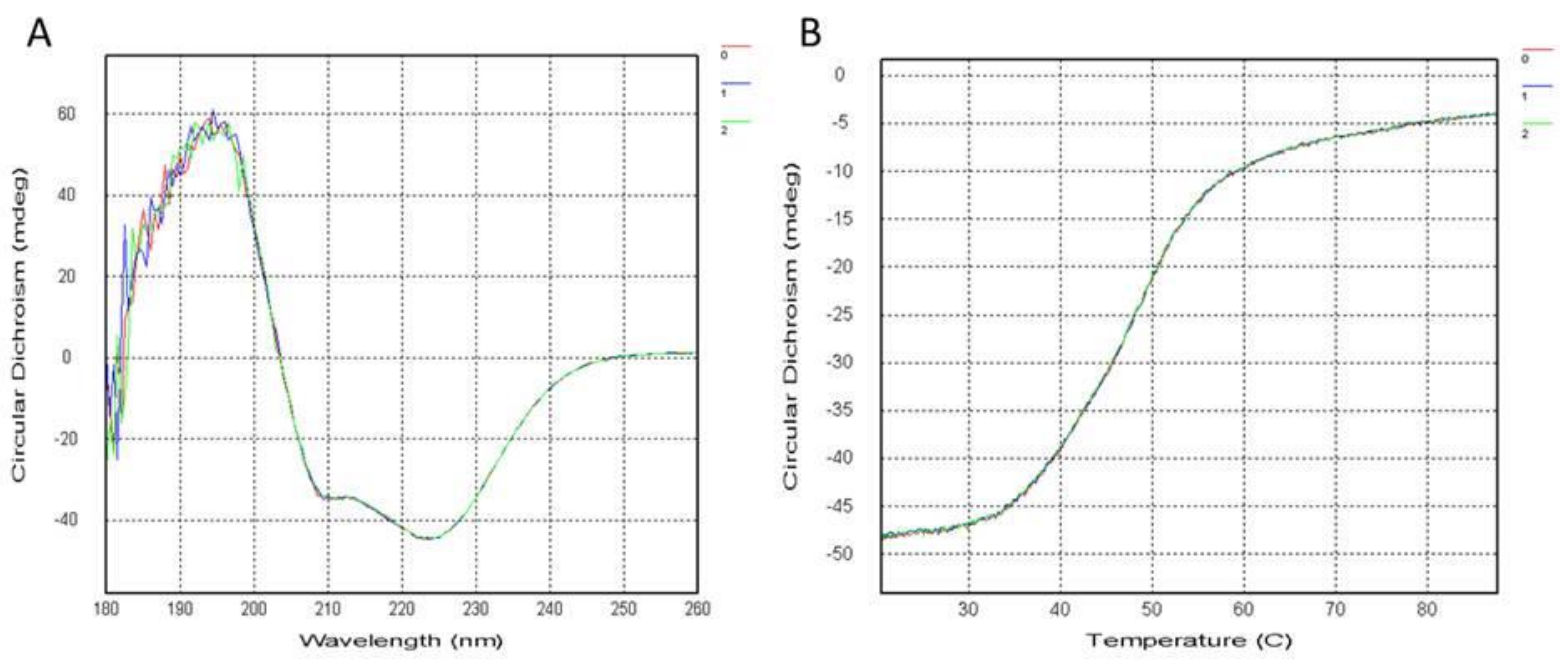

Figure 3.3.3.: CD spectroscopy of the SCOC(78-159)-FEZ1 (225-295) complex. $10 \mu \mathrm{M}$ SCOC (78159) FEZ1 (225-295) was dialyzed overnight in $20 \mathrm{mM}$ HEPES pH 7.5 and $200 \mathrm{mM} \mathrm{NaF}$. A: CD Spectrum of SCOC (78-159) FEZ1 (225-295) from 180 to $260 \mathrm{~nm}$. B: Melting curve for SCOC (78159) FEZ1 (225-295) measured at $222 \mathrm{~nm}$ from 20 to $90{ }^{\circ} \mathrm{C}$. 


\subsubsection{Stoichiometric analysis of the SCOC(78-159)-FEZ1 (225-295) complex by SEC-}

\section{MALLS}

The composition of the SCOC (78-159) FEZ1 (225-295) complex was investigated by SECMALLS measurements. After separation by size on a Superdex 10/300 GL samples were loaded into a MALLS detector (Fig. 3.3.4.). MALLS gives the molecular weight of a protein independent of its shape and can therefore be used for the determination of molecular weights of non-globular proteins. The calculated molecular mass of Strep-SCOC (78-159) is 11.004 kDa. SEC-MALLS measurements by $\mathrm{C}$. Behrens gave $26.3 \pm 0.3 \mathrm{kDa}$, which correlates to a dimer [12]. The calculated molecular mass of His-FEZ1 (225-295) is $10.256 \mathrm{kDa}$. SECMALLS measurements of the Strep-SCOC (78-159)-His-FEZ1 (225-295) complex give a molecular mass of $43.9 \pm 0.2 \mathrm{kDa}$, which is consistent with a stoichiometry of $1: 1$ assuming that both proteins are dimers (Table 3.3.1.).

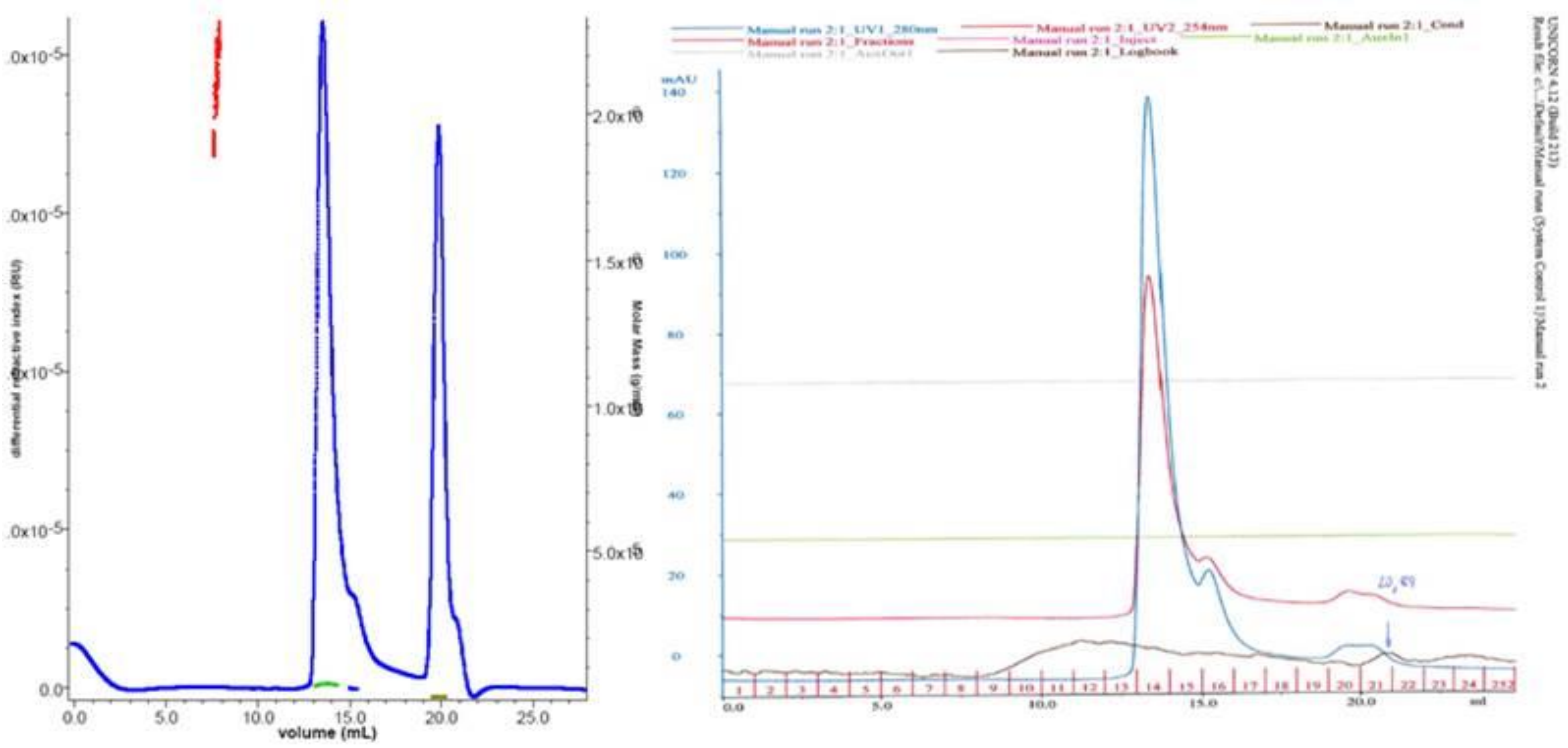

Figure 3.3.4.: SEC-MALLS of SCOC(78-159)-FEZ1(225-295) complex. Left chromatogram: Peaks correspond to size exclusion chromatography elution volume (x-axis). Horizontal lines indicate differential refractive index (y-axis). Measured value was only considered for the main peak (green line). Right chromatogram: Size exclusion chromatography using a Superdex 200 10/300 GL column prior to MALLS measurements. Blue curve: UV $280 \mathrm{~nm}$, red curve: UV $254 \mathrm{~nm}$. 
Table 3.3.1.: SEC-MALLS measurements of SCOC(78-159)-FEZ1(225-295) complex. Listed are the average molar mass measured by SEC-MALLS and the theoretical mass calculated from the protein sequence including Tags. The average molar weight of SCOC (78-159) was measured by Dr. C. Behrens [12].

\begin{tabular}{|c|c|c|c|}
\hline Protein & SCOC(78-159) & FEZ1(225-295) & $\begin{array}{c}\text { SCOC(78-159) } \\
\text {-FEZ1 (225-295) } \\
\text { complex }\end{array}$ \\
\hline $\begin{array}{c}\text { Average molar weight } \\
(\mathrm{g} / \mathrm{mol})\end{array}$ & $\begin{array}{c}2.63 \times 10^{4}( \pm 0.3 \%) \\
\text { (Dr. C. Behrens) }\end{array}$ & - & $4.439 \times 10^{4}( \pm 0.010 \%)$ \\
\hline $\begin{array}{c}\text { Theoretical molar } \\
\text { weight for a monomer } \\
(\mathrm{g} / \mathrm{mol})\end{array}$ & $1.1 \times 10^{4}$ & $1.026 \times 10^{4}$ & - \\
\hline
\end{tabular}

\subsubsection{Crystallization of the SCOC-FEZ1 complex}

\subsubsection{SCOC(78-159)-FEZ1(227-290) crystallization}

Purified SCOC(78-159)-FEZ1(227-290) was used for crystallization with commercial crystallization screens in 96-well plates. Crystals in different shapes were obtained in different conditions in MIDAS and ProComplex screens (Fig. 3.3.5.). Crystals were soaked in cryoprotectant and flash cooled in liquid nitrogen. Crystals were measured at beamline X10SA at the Swiss Light Source (SLS) and diffracted up to $3.3 \AA$. Indexing gave the same space group and cell dimension as SCOC crystals and structure determination using molecular replacement with SCOC ccd (PDB code: 4BWD) resulted in the already solved structure. 
A

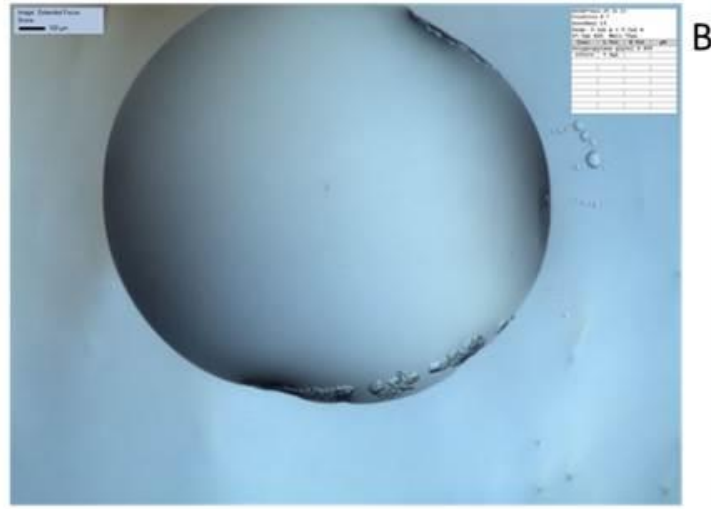

$\mathrm{C}$

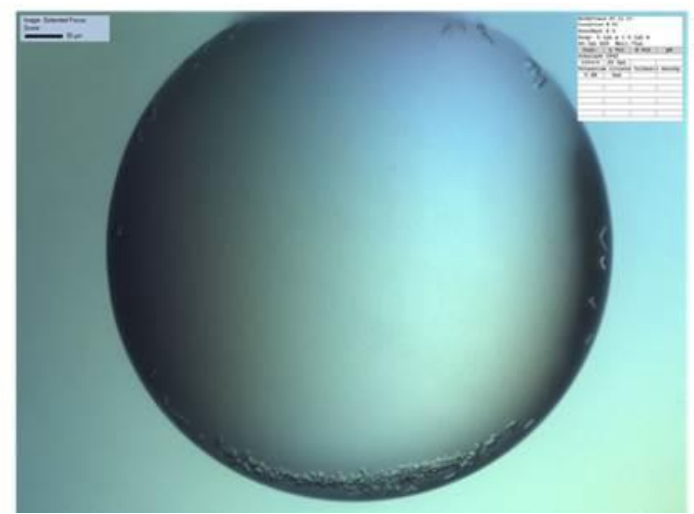

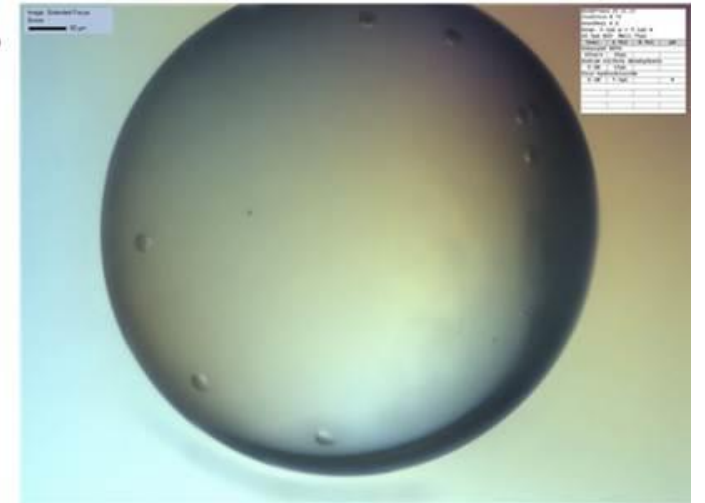

$\mathrm{D}$

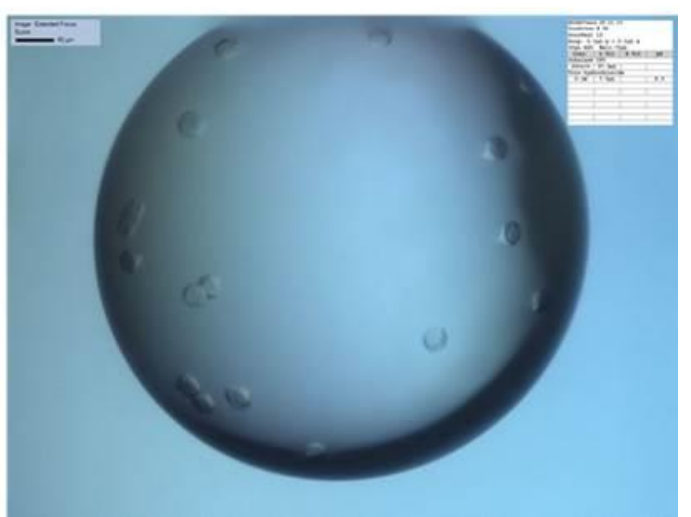

Figure 3.3.5.: Initial crystals from SCOC(78-159)-FEZ1(227-290) crystallization. Letters A-D correspond to the crystallization conditions stated in table 3.3.2.

Table 3.3.2.: Initial crystallization conditions for SCOC(78-159)-FEZ1(227-290) crystallization from 96-well plate screening

\begin{tabular}{|c|l|}
\hline Crystals & Buffer composition \\
\hline A & $10 \%(\mathrm{v} / \mathrm{v})$ Polypropylene glycol P 400 \\
\hline B & $0.1 \mathrm{M}$ Tris $\mathrm{pH} 8,0.2 \mathrm{M}$ sodium sulfate decahydrate, $20 \%(\mathrm{w} / \mathrm{v})$ Sokalan ${ }^{\circledR}$ HP56 \\
\hline C & $0.2 \mathrm{M}$ potassium citrate tribasic monohydrate, $15 \%$ (v/v) Sokalan ${ }^{\circledR}$ CP42 \\
\hline $\mathrm{D}$ & $0.1 \mathrm{M}$ Tris $\mathrm{pH} 8.5,25 \%(\mathrm{v} / \mathrm{v})$ Sokalan ${ }^{\circledR} \mathrm{CP} 5$ \\
\hline
\end{tabular}

\subsubsection{SCOC(78-159)-FEZ1(225-295) crystallization}

After optimization of the purification protocol the SCOC(78-159)-FEZ1(225-295) complex was used for crystallization. Using the more stable complex new crystal forms were obtained in commercial crystallization screens. 96-well plates with $200 \mathrm{nl}$ sitting drops at $20{ }^{\circ} \mathrm{C}$ gave several crystallization hits (Fig. 3.3.6.). Only crystals from PEG 20000 (Fig. 3.3.6. D) and isopropanol conditions (Fig. 3.3.6. B) could be reproduced on 24 well plates and were a target for further optimization. 
A

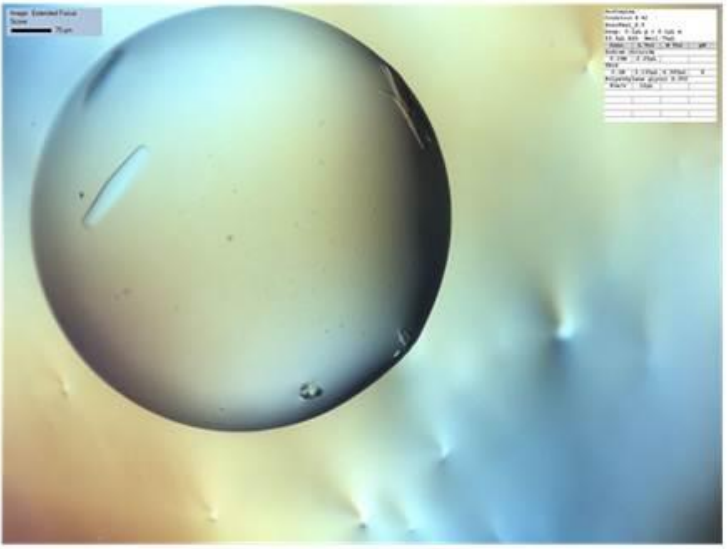

$\mathrm{C}$

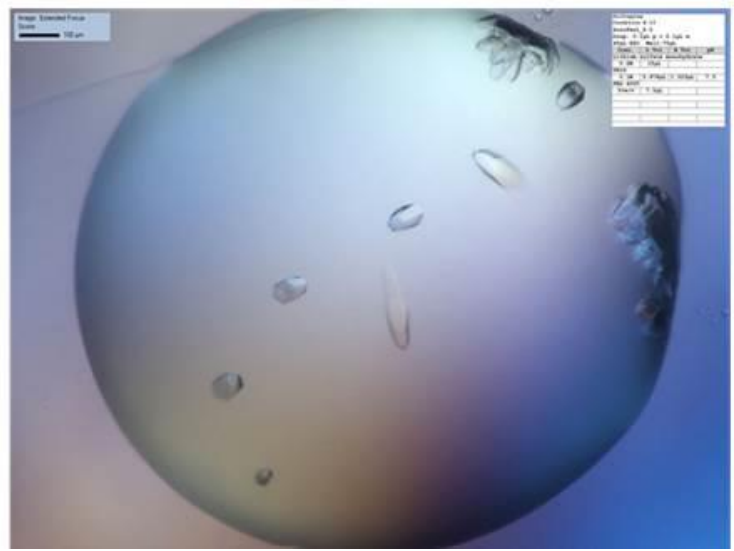

B

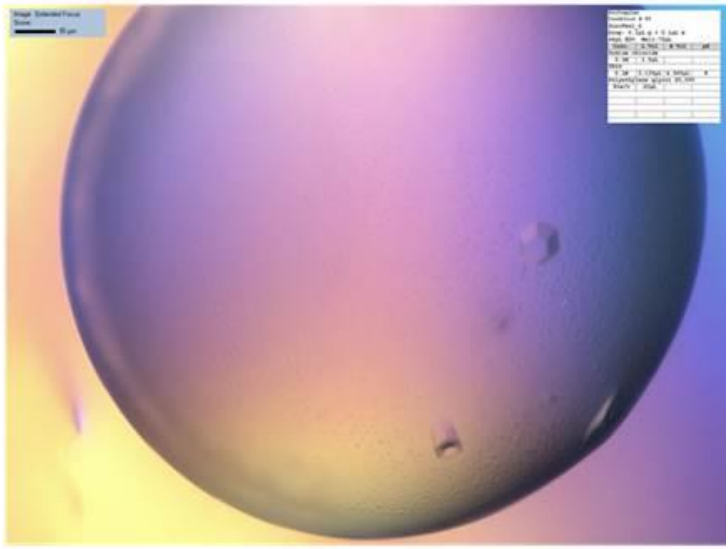

$\mathrm{D}$

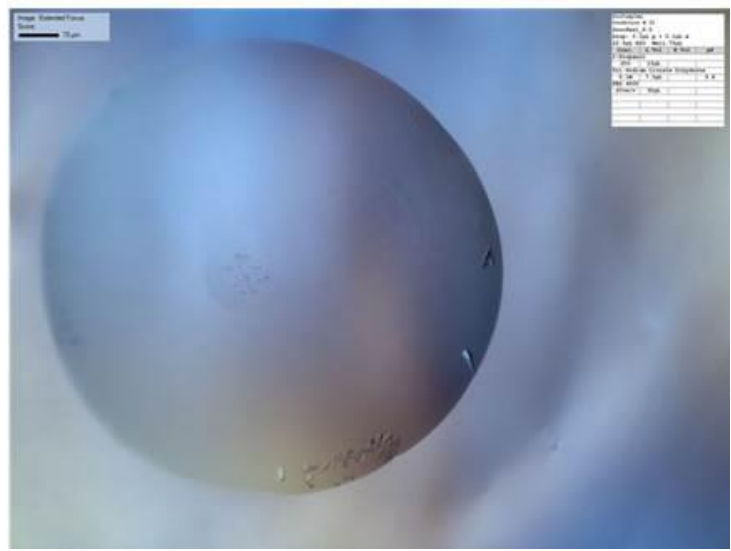

Figure 3.3.6.: Initial crystals from SCOC(78-159)-FEZ1(225-295) crystallization. Letters A-D correspond to the crystallization conditions stated in table 3.3.3.

Table 3.3.3.: Initial crystallization conditions for SCOC(78-159)-FEZ1(225-295) crystallization from 96-well plate screening

\begin{tabular}{|c|l|}
\hline Crystals & Buffer composition \\
\hline A & $0.1 \mathrm{M}$ Tris $\mathrm{pH} 8,0.15 \mathrm{M}$ sodium chloride, $8 \%(\mathrm{w} / \mathrm{v})$ PEG 6000 \\
\hline B & $0.1 \mathrm{M}$ Tris $\mathrm{pH} 8,0.1 \mathrm{M}$ sodium chloride, $8 \%(\mathrm{w} / \mathrm{v})$ PEG 20000 \\
\hline C & $0.1 \mathrm{M}$ Tris $\mathrm{pH}$ 7.5, 0.2 M lithium sulfate monohydrate, 5 \% (w/v) PEG 4000 \\
\hline D & $\begin{array}{l}20 \%(\mathrm{v} / \mathrm{v}) \text { 2-Propanol, 0.1 M tri-sodium citrate dihydrate } \mathrm{pH} 5.6, \\
20 \%(\mathrm{w} / \mathrm{v}) \text { PEG 4000 }\end{array}$ \\
\hline
\end{tabular}

In order to get data for SAD phasing methionine mutants of SCOC- FEZ1 complex were used for crystallization trials as well. Purified selenomethionine labelled proteins were used in the same crystallization conditions as native complex. SCOC (78-159) L105M - FEZ1 (225-295) L273M complex crystallized in the same conditions as the native complex. The FEZ1 (225295) L251M mutant did not crystallize. 


\subsubsection{Optimization of SCOC(78-159)-FEZ1(225-295) crystals in PEG 20000 conditions}

Crystals obtained from commercial ProComplex screen condition 65 (0.1 M Tris pH 8, $0.1 \mathrm{M}$

Sodium chloride, $8 \%$ (w/v) PEG 20000) had a nice shape and promising size. Initial crystals diffracted up to $4.5 \AA$. To further optimize these crystals different methods were applied as summarized in table 3.3.4..

Table 3.3.4.: Optimization approaches for SCOC(78-159)-FEZ1(225-295) crystallization

\begin{tabular}{|l|l|l|}
\hline Optimization method & Influence on morphology & Influence on diffraction \\
\hline Grid screen & Larger and more defined crystals & $\begin{array}{l}\text { Enhanced diffraction from initial } \\
4.5 \AA \text { A to 3.4 A }\end{array}$ \\
\hline Streak seeding & Faster crystallization & $\begin{array}{l}\text { No further influence on } \\
\text { diffraction }\end{array}$ \\
\hline $\begin{array}{l}\text { Ratio of protein to } \\
\text { precipitant }\end{array}$ & Larger and more defined crystals & $\begin{array}{l}\text { No further influence on } \\
\text { diffraction }\end{array}$ \\
\hline Additive Screen & Overnucleation & Not tested \\
\hline In-situ proteolysis & Different shape & Less diffraction \\
\hline $\begin{array}{l}\text { Cryoprotectant screen } \\
\text { Molecular Dimensions } \\
\text { CryoProtX' }\end{array}$ & Less ice on crystals & No influence on diffraction \\
\hline $\begin{array}{l}\text { Tag removal by Thrombin } \\
\text { cleavage }\end{array}$ & $\begin{array}{l}\text { Crystals very similar to native } \\
\text { crystals }\end{array}$ & Loss of diffraction \\
\hline Manual Dehydration & Crystals break or turn yellow & Loss of diffraction \\
\hline $\begin{array}{l}\text { Oil to control vapor } \\
\text { diffusion rate }\end{array}$ & No difference & No influence on diffraction \\
\hline $\begin{array}{l}\text { Drop size } \\
\text { Ratio of protein to } \\
\text { precipitant }\end{array}$ & Crystals grew smaller or same size & No influence on diffraction \\
\hline $\begin{array}{l}\text { Crystallization at } 4{ }^{\circ} \mathrm{C} \\
\text { Crystal did not grow until plate was } \\
\text { moved to } 20^{\circ} \mathrm{C}\end{array}$ & No influence on diffraction \\
\hline
\end{tabular}

\section{Grid screen}

First commercial screen conditions were reproduced using solutions prepared with ultrapure chemicals. The condition was optimized with grid screens on a $24-w e l l$ plate using hanging drops. Drop size was up scaled from $200 \mathrm{nl}$ to $2 \mu \mathrm{l}$. For grid screening the $\mathrm{pH}$ and precipitant concentration of the crystallization solution was varied along row and column. Crystals in 24well hanging drop plates appeared after 5-7 days and grew for 5 days to a larger size than in 96-well sitting drop plates. Protein concentrations from 5 to $15 \mathrm{mg} / \mathrm{ml}$ were tested and biggest crystals were observed for the highest protein concentrations (Fig. 3.3.7.). 
A

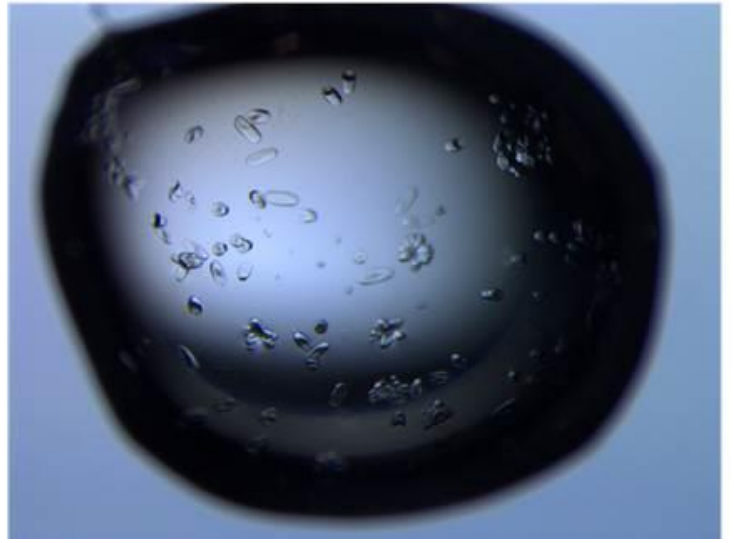

B

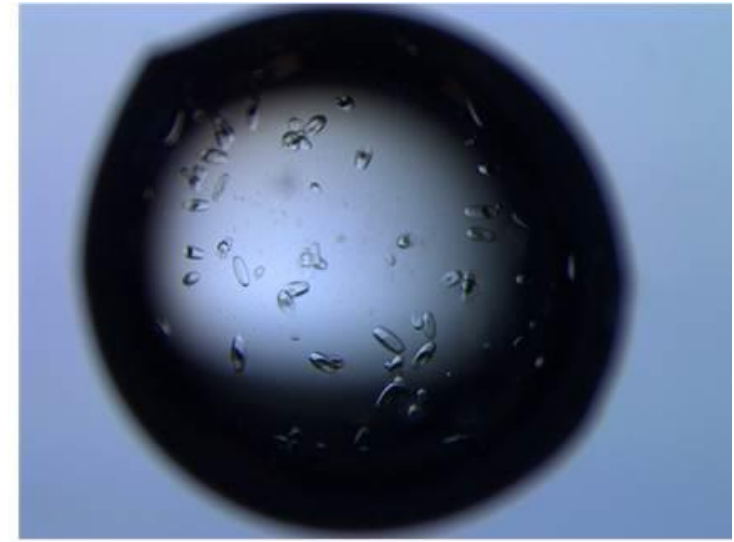

Figure 3.3.7.: Elongated hexagonal SCOC(78-159)-FEZ1(225-295) crystals from grid screen optimization. 24-well Linbro plate. Drop size $2 \mu$ l. Letters A-B correspond to the crystallization conditions stated in table 3.3.5.

Table 3.3.5.: Crystallization conditions for SCOC(78-159)-FEZ1 (225-295) crystals from grid screen optimization

\begin{tabular}{|c|l|}
\hline Condition & Buffer composition \\
\hline A & 0.1 M Tris pH 7.5, 0.1 M sodium chloride, $10 \%$ (w/v) PEG 20000 \\
\hline B & 0.1 M Tris pH 8.0, 0.1 M sodium chloride, 10 \% (w/v) PEG 20000 \\
\hline
\end{tabular}

\section{Streak seeding}

While refining crystallization conditions in 24-well plates, streak seeding using small crushed crystal that provide nucleation sites were tested. Using streak seeding crystals grew faster and less multiple crystals were observed.

\section{Ratio of protein to precipitant}

Different ratios of protein to precipitant were tested to further improve crystal growth. Best results were observed for crystals grown in $2 \mu \mathrm{l}$ protein of $15 \mathrm{mg} / \mathrm{ml}$ with $1 \mu \mathrm{l}$ of precipitant solution (Fig. 3.3.8.). 

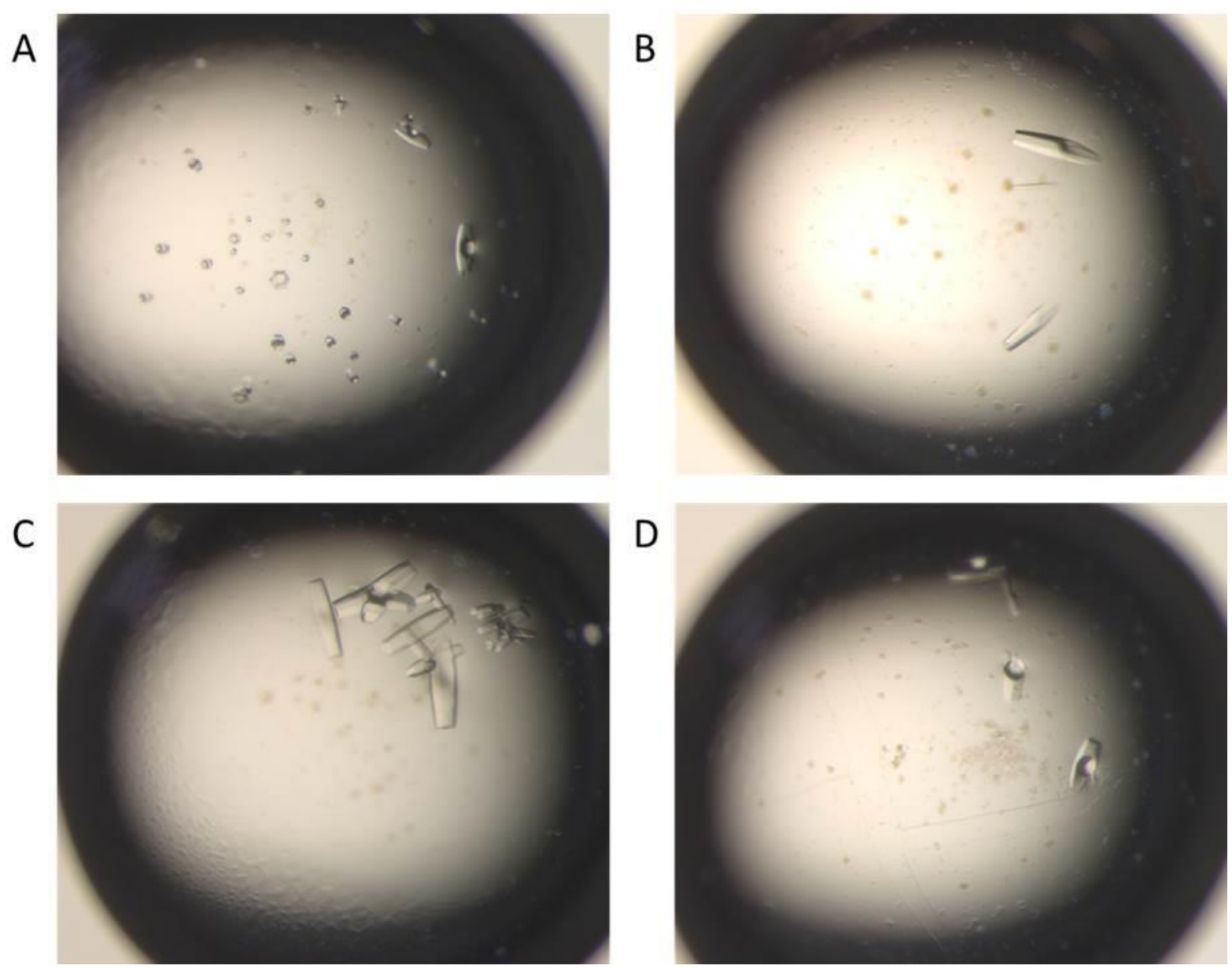

Figure 3.3.8.: Elongated hexagonal SCOC(78-159)-FEZ1(225-295) crystals from drop size optimization. Optimized crystals after Grid Screen refinement using streak seeding and a drop size of $2 \mu \mathrm{l}$ protein $15 \mathrm{mg} / \mathrm{ml}$ and $1 \mu \mathrm{l}$ precipitant solution. 24-well Linbro plate. Letters A-D correspond to the crystallization conditions stated in table 3.3.6.

Table 3.3.6.: Crystallization conditions for $\operatorname{SCOC(78-159)-FEZ1(225-295)~crystals~from~drop~}$ size optimization

\begin{tabular}{|c|l|}
\hline Condition & Buffer composition \\
\hline A & $0.1 \mathrm{M}$ Tris $\mathrm{pH} 8,0.1 \mathrm{M}$ sodium chloride, 6\% (w/v) PEG 20000 \\
\hline B & $0.1 \mathrm{M}$ Tris $\mathrm{pH} 8,0.1 \mathrm{M}$ sodium chloride, $8 \%$ (w/v) PEG 20000 \\
\hline C/D & $0.1 \mathrm{M}$ Tris $\mathrm{pH}$ 7.5, 0.1 M sodium chloride, $8 \%(\mathrm{w} / \mathrm{v})$ PEG 20000 \\
\hline
\end{tabular}

\section{Additive Screen}

The Hampton Additive Screen was used to find additives that could optimize crystallization. Therefore, two drops composed of $1.5 \mu 115$ or $10 \mathrm{mg} / \mathrm{ml}$ protein complex and $1.5 \mu \mathrm{l}$ crystallization solution (0.1 M Tris pH 7.5, 0.1 M Sodium chloride, $10 \%$ (w/v) PEG 20000) was pipetted on a 24-well Linbro plate. $0.3 \mu \mathrm{l}$ of each additive was added respectively. Additives that further optimized crystallization were not found. Instead a shower of very 
small crystals was observed in many conditions (Fig. 3.3.9. A) or crystals did not grow as large as without additives (Fig. 3.3.9. B).
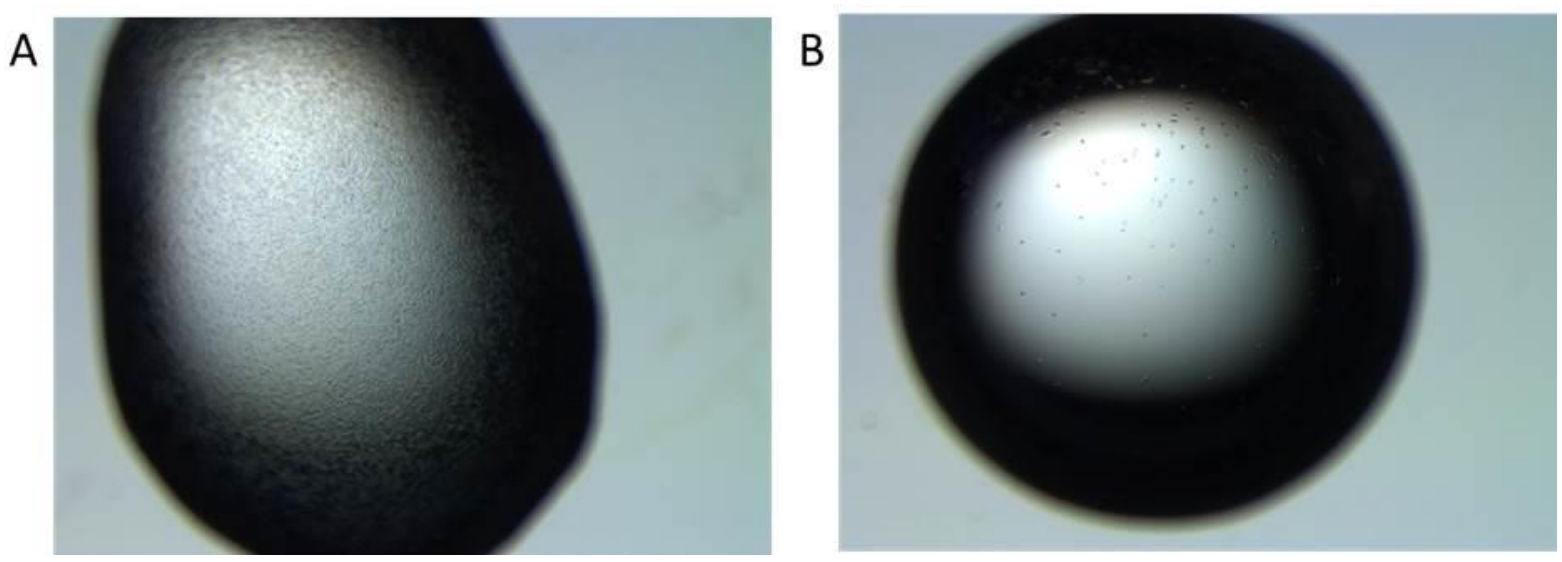

Figure 3.3.9.: SCOC(78-159)-FEZ1(225-295) crystals from additive screen. Precipitant contained 0.1 M Tris pH 7.5, 0.1 M Sodium chloride, $10 \%$ (w/v) PEG 20000 A: Additive 0.1 M Copper(II) chloride dihydrate resulted in a crystal shower. B: Crystal grew only to very small size with additive $0.1 \mathrm{M}$ Zinc chloride.

\section{Manual Dehydration}

One reason for poor diffraction of crystals is loose packing of protein molecules in the crystal lattice. Dehydration can reduce the solvent content in the crystal, lead to tighter packing and thereby improve the diffraction limit. Dehydration was approached by the step-wise addition of increasing PEG 400 concentrations $(5-25 \%)$ or of 4 M Trimethylamine oxide (TMAO) as described in [52]. Crystals were transferred from 24-well Linbro plates into fresh drops of mother liquor and increasing dehydration conditions. Crystals were equilibrated at each step for $\sim 5$ minutes. Most crystals cracked, vanished or turned yellow during this treatment. Crystals that remained intact after step-wise dehydration were cryoprotected and flash-cooled in liquid nitrogen. Another approach was to transfer coverslips with crystals into a new 24well plate with crystallization conditions of increasing PEG or conditions as described in [52]. Crystals were equilibrated over the fresh mother liquor for 12 hours. Crystals appeared yellow and jelly-like after this dehydration approach (Fig. 3.3.10.). Crystals were cryoprotected and flash-cooled in liquid nitrogen. All crystals from manual dehydration approaches did not diffract. 

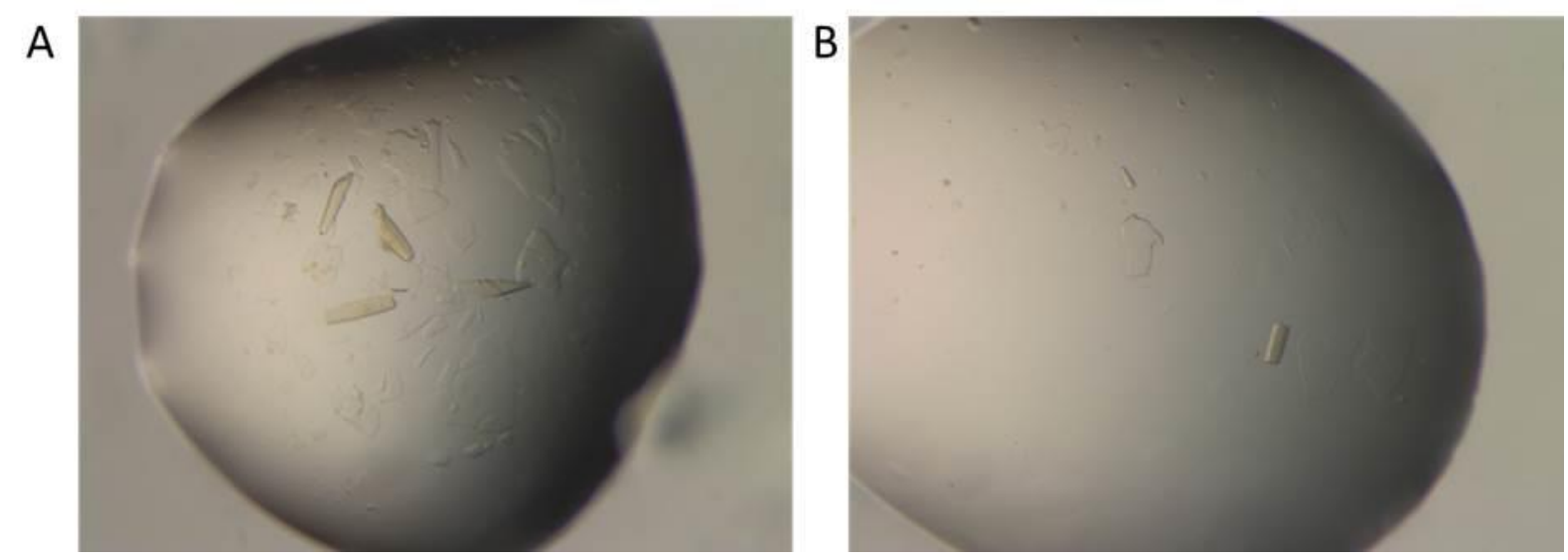

Figure 3.3.10.: SCOC(78-159)-FEZ1(225-295) crystals after manual dehydration. Crystals turned yellow after manual dehydration. Crystals grew in $0.1 \mathrm{M}$ Tris $\mathrm{pH} 8,0.1 \mathrm{M}$ Sodium chloride, $8 \%(\mathrm{w} / \mathrm{v})$ PEG 20000. A: Step-wise transfer to 4 M TMAO or B: $20 \%$ PEG 400 additional to the mother liquor.

\section{In situ proteolysis crystallization of SCOC(78-159)-FEZ1(225-295)}

Flexible regions of a protein can hamper crystallization and limited proteolysis can be used to crop flexible elements and thus enhance crystallization. Because in situ proteolysis was used to solve the structure of the SCOC ccd, limited proteolysis was also used for the SCOC(78159)-FEZ1(225-295) to identify stable fragments. The complex was digested with twelve different proteases from the Proti-Ace Kit $1 \& 2$ (Hampton Research) at $37{ }^{\circ} \mathrm{C}$. The complex was incubated for $60 \mathrm{~min}$ at $37^{\circ} \mathrm{C}$ without addition of protease as a control. Samples were taken at different time points of protease incubation. Analysis by Schägger gels showed that most proteases digested the complex resulting in bands with lower molecular weight (Fig. 3.3.11. A). PE, $\alpha-C, T R$ and $S U$ were used in higher concentrations to further investigate their effects (Fig. 3.3.11. B). 


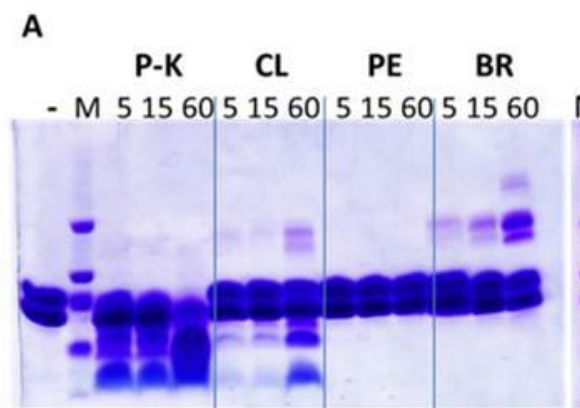

B

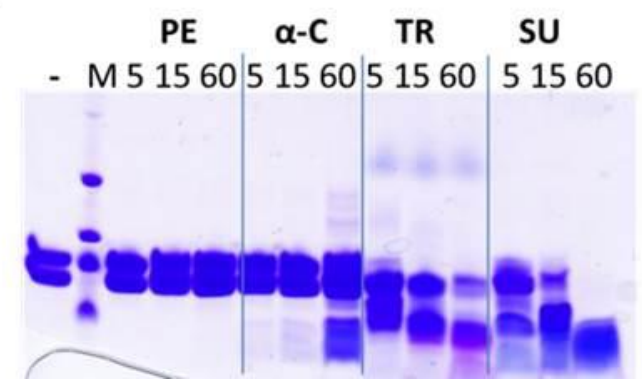

TH $\quad$ A-E $\quad \alpha-C \quad$ TR
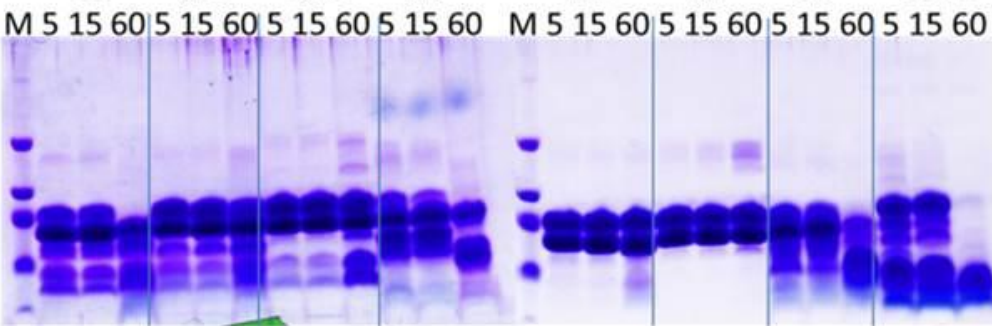

C

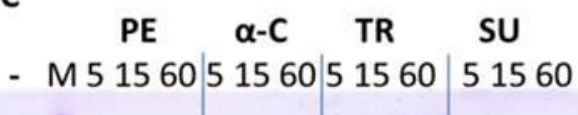

Figure 3.3.11.: Limited proteolysis of SCOC(78-159)-FEZ1(225-295) complex. A: $10 \mathrm{mg} / \mathrm{ml}$ SCOC (78-159)-FEZ1 (225-295) complex was incubated without (-) or with 1:1000 protease for 5, 10, 15 minutes at $37{ }^{\circ} \mathrm{C}$. Protease assay was repeated with B: 1:500 and C: $1: 200$ proteases. P-K: Proteinase-K, CL: Clostripain, PE: Pepsin, TH: Thermolysin, BR: Bromelain, A-E: Actinase E, $\alpha-\mathrm{C}$ : $\alpha$-Chymotrypsin, TR: Trypsin, EL: Elastase, PA: Papain, SU: Substilisin, EG-C: Endoproteinase GluC. M: Marker.

The most promising proteases $\mathrm{P}-\mathrm{K}, \mathrm{TH}, \mathrm{A}-\mathrm{E}, \alpha-\mathrm{C}, \mathrm{TR}, \mathrm{SU}$ and EG-C were used for in situ crystallization in PEG 20.000 conditions, as a control $\mathrm{H}_{2} \mathrm{O}$ was added instead of protease. Crystals grew after treatment with TH, P-K and $\alpha-\mathrm{C}$ (Fig. 3.3.12.). Crystals from in situ crystallization with TH resulted in somewhat different shaped crystals. Crystals were soaked in cryoprotectant and flash cooled in liquid nitrogen. Crystals were measured at beamline X10SA at the Swiss Light Source (SLS) but no improved diffraction was observed. 

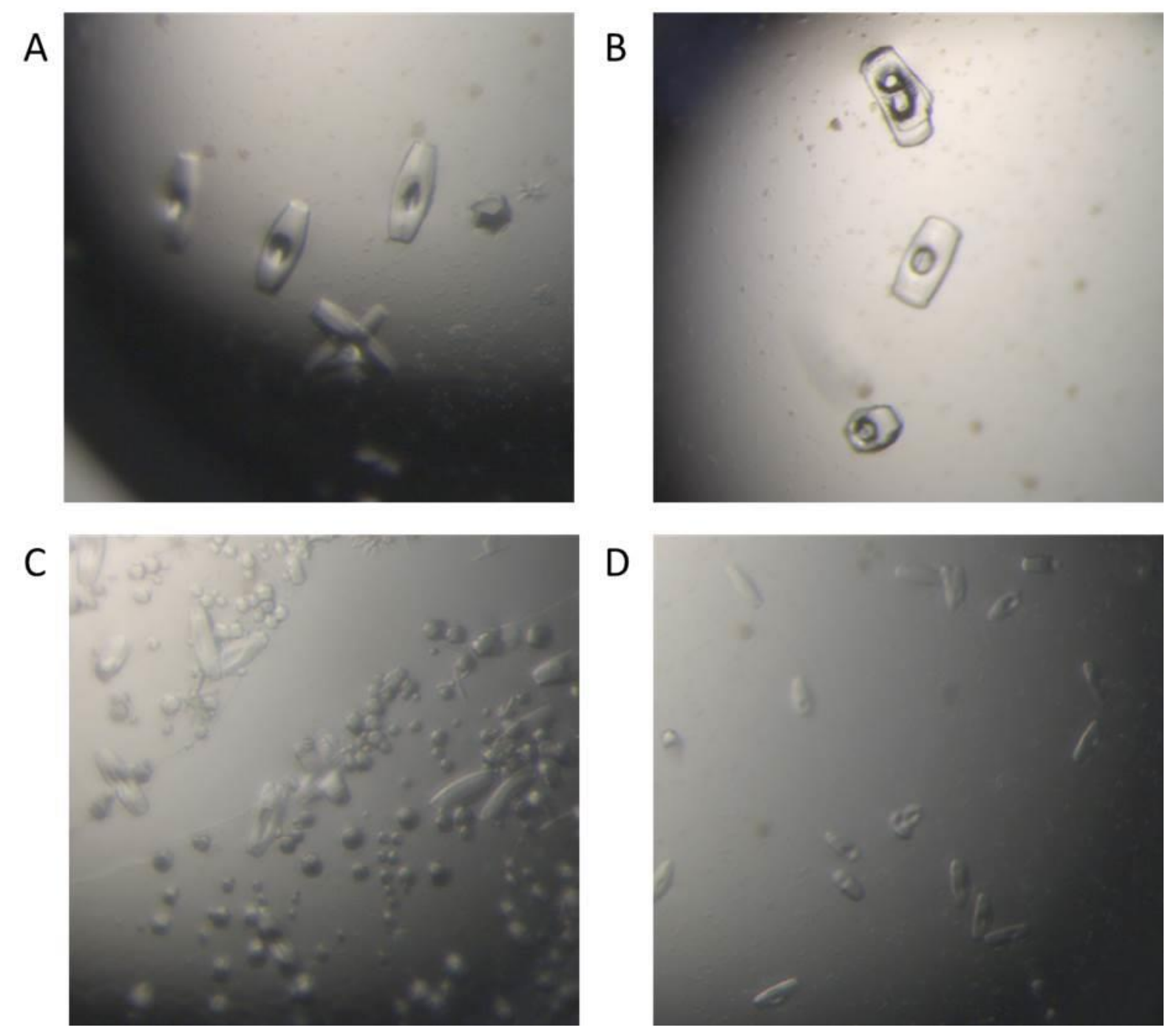

Figure 3.3.12.: In situ crystallization of SCOC(78-159)-FEZ1(225-295). Crystals grew in $0.1 \mathrm{M}$ $\mathrm{NaCl}, 0.1 \mathrm{M}$ Tris pH 8, $10 \%$ PEG 20 000. Drops: $2 \mu \mathrm{l}$ protein $15 \mathrm{mg} / \mathrm{ml}+1 \mu \mathrm{l} \mathrm{ML}+0.3 \mu \mathrm{l}$ protease $1 \mu \mathrm{g} / \mu \mathrm{l}$ or $\mathrm{H}_{2} \mathrm{O}$. A: $\mathrm{H}_{2} \mathrm{O}$ B: TH, C: P-K D: $\alpha-\mathrm{C}$.

\section{Automated Dehydration}

Because unit cell dimensions of SCOC(78-159)-FEZ1(225-295) crystals from PEG 20.000 crystals indicated a high solvent content another dehydration approach was tested. Using a humidity control device the dehydration of crystals can be controlled [16]. Because this device can be attached to a synchrotron beamline effects on diffraction can be directly observed during the dehydration. For automated dehydration the $\mathrm{HC} 1 \mathrm{c}$ crystal humidifier at beamline BL14.3 (BESSY, Helmholtz Zentrum Berlin) was used with technical support of Dr. Manfred S. Weiss. The HC1c is equipped with an open air stream at room temperature with adjustable relative humidity $(\mathrm{RH})$ of $45-99.7 \%$. Prior to the experiment the equilibrium relative humidity $(\mathrm{RH})$ was calculated to be $99.8 \%$ for crystals grown in $0.1 \mathrm{M} \mathrm{NaCl}, 0.1 \mathrm{M}$ Tris pH 8, $10 \%$ PEG 20000 with the following equation: 


$$
R H=\frac{1}{1+\frac{18 x}{(1-x) m}} \exp \left\{(1-m / n) /\left(1+\frac{(1-x) m}{18 x}\right)\right\}
$$

Crystals were mounted on mesh loops at the beamline. Excess liquid was removed using filter paper. Crystals were then mounted at room temperature and a first test shot was taken at the starting point (Fig. 3.3.13. A). The relative humidity was then stepwise reduced. When the relative humidity was reduced to $97.5 \%$ the diffraction was slightly enhanced (Fig. 3.3.13. B). At $95 \%$ RH diffraction started to decrease but could be rescued by increasing the RH to $97.5 \%$ again. At $95 \%$ RH crystals almost completely lost diffraction. The optimal RH for SCOC(78-159)-FEZ1(225-295) crystals was $97.5 \%$. Crystals were dehydrated to $97.5 \% \mathrm{RH}$ and flash cooled in liquid N2 without addition of a cryoprotectant. Frozen crystals were taken to beamline X10SA at the SLS to measure a full data set. Without cryoprotectant crystals were covered in ice and did not diffract.
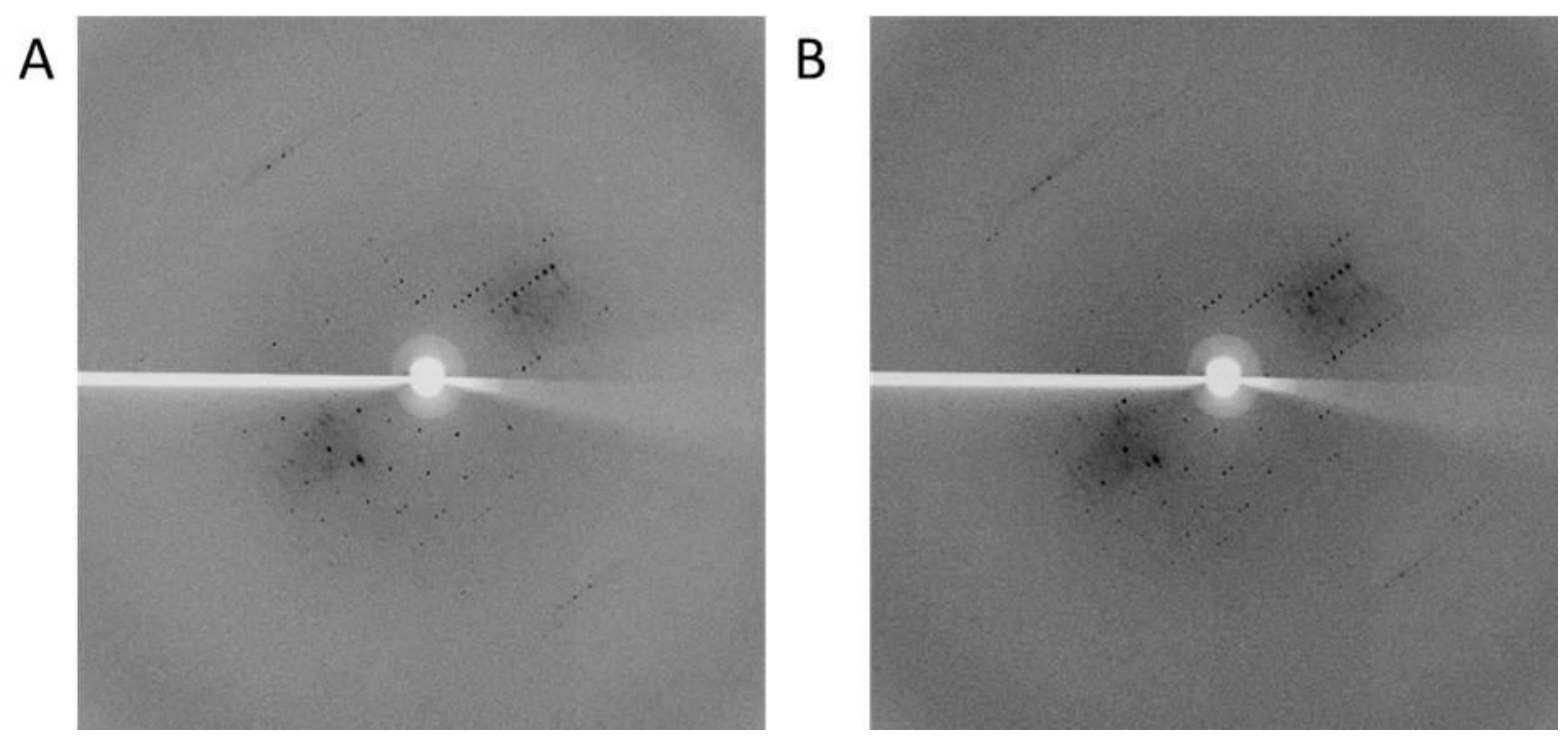

Figure 3.3.13.: Diffraction pattern of SCOC(78-159)-FEZ1(225-295) crystals at beamline BL14.3 (BESSY, Helmholtz Zentrum Berlin). Diffraction was measured at A: $99.8 \%$ relative humidity (RH) and B: $97.5 \%$ RH.

\subsubsection{X-ray data collection and analysis of SCOC(78-159)-FEZ1(225-295) crystals} from PEG 20.000 conditions

SCOC(78-159)-FEZ1(225-295) crystals were soaked in crystallization solution supplemented with cryoprotectant before flash cooling in liquid nitrogen. Data from native or selenomethionine labeled crystals were collected at $100 \mathrm{~K}$ at beamline X10SA (Swiss Light Source, Paul Scherrer Institute, Villigen, Switzerland). A fluorescence spectrum was recorded 
tor selenomethionine labeled crystals to define the selenium absorption spectrum (Fig. 3.3.14.). The wavelength was tuned to the selenium peak prior to data collection.

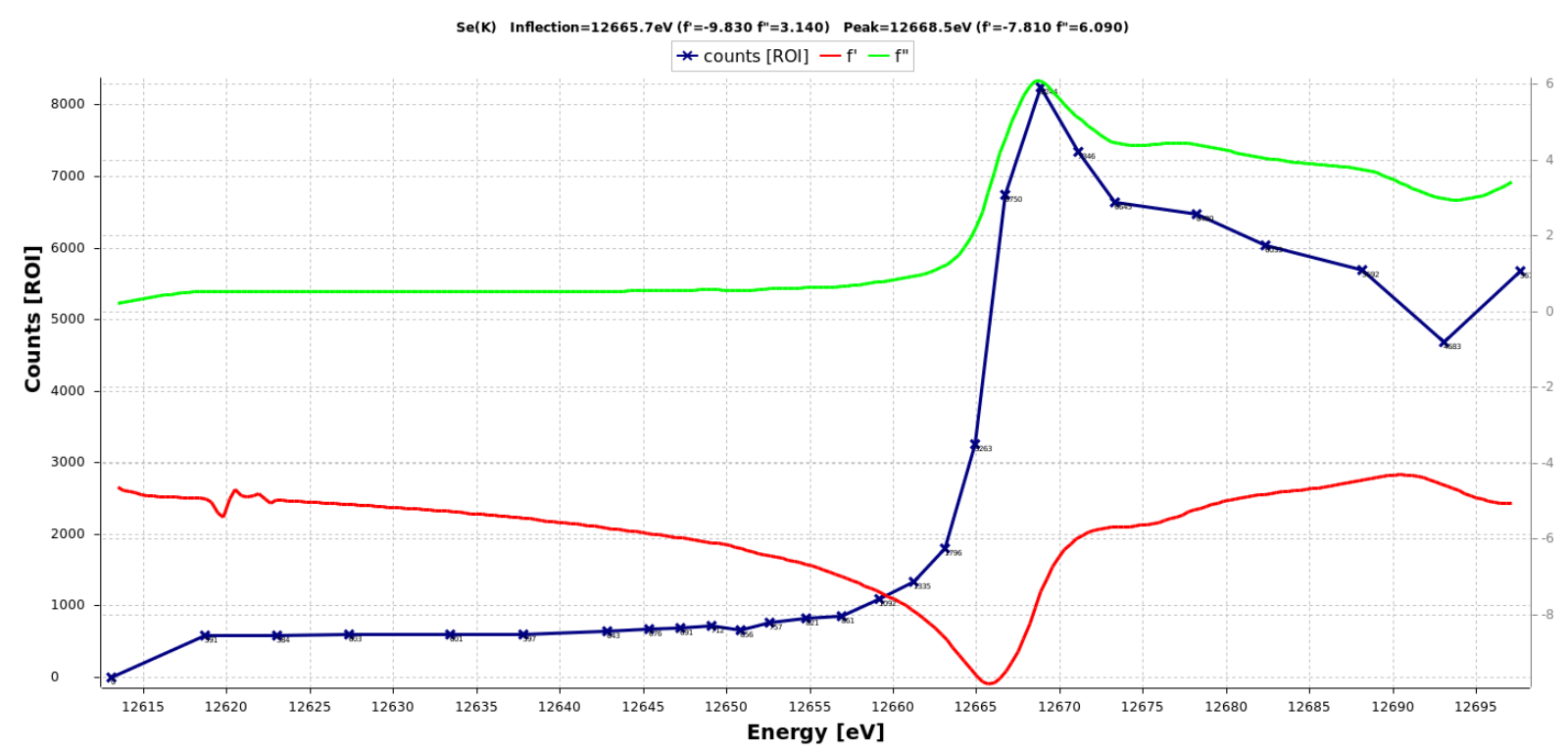

Figure 3.3.14.: Fluorescence spectrum of selenomethionine labeled $\operatorname{SCOC}(78-159)-F E Z 1(225-$ 295) PEG 20000 crystals. Crystals grew in PEG 20000 conditions. Spectrum measured at beamline X10SA (Swiss Light Source, Paul Scherrer Institute, Villigen, Switzerland). Counts in the region of interest (ROI) are recorded as a function of energy (in eV). Blue curve: Counts (ROI), red curve: fitted $f^{\prime}$, green curve: fitted: $f^{\prime \prime}$.

Complete datasets with 360 degrees total oscillation were collected at the Se peak wavelength or at $1 \AA$. The hexagonal SCOC(78-159)-FEZ1(225-295) crystals from PEG 20.000 conditions revealed a hexagonal diffraction pattern with a maximum diffraction of $3.4 \AA$ (Fig. 3.3.15.).
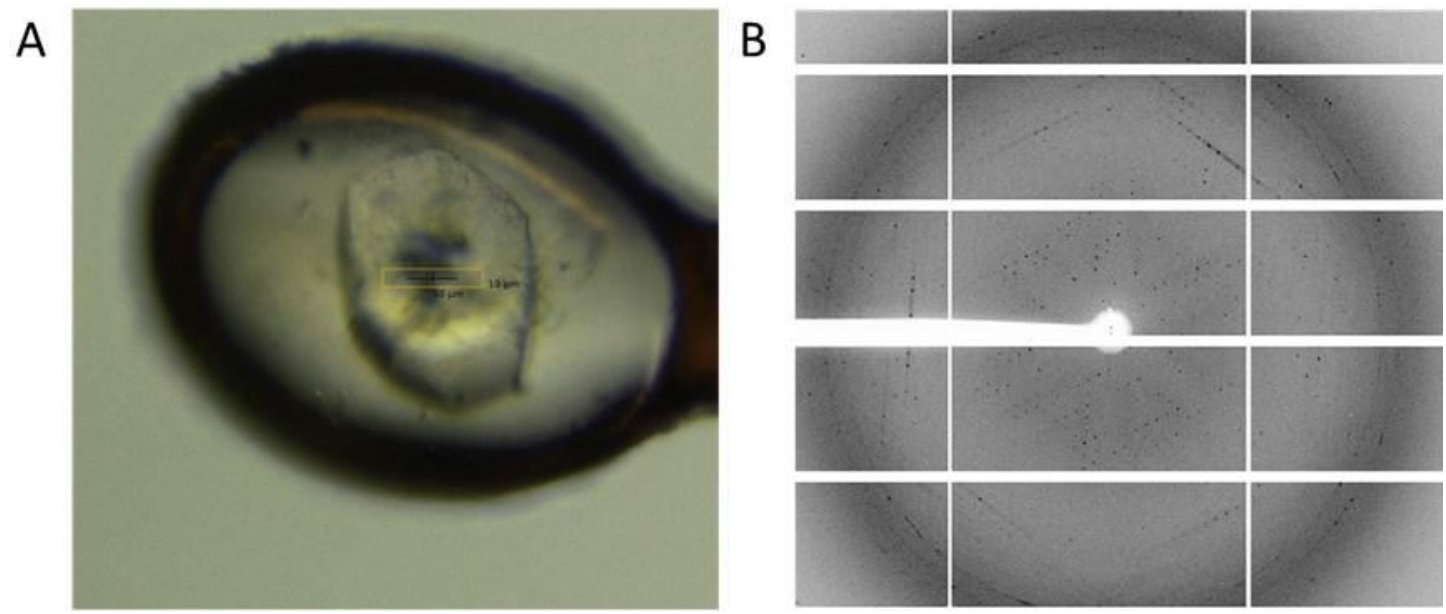

Figure 3.3.15.: SCOC(78-159)-FEZ1(225-295) crystals grown in PEG 20000 conditions at beamline X10SA (Swiss Light Source, Paul Scherrer Institute, Villigen, Switzerland). A: Crystal mounted at the beamline. The yellow square indicates the X-ray beam and its size with $10 \times 50 \mu \mathrm{m}$. B: Diffraction pattern observed from the same crystal. 
Data were processed with input values specific for the PILATUS 6M detector using the XDS software package. Consistent with the hexagonal shape of the crystals and diffraction pattern the crystals are hexagonal primitive. The enantiomorphic space groups $\mathrm{P}_{1} 22$ or $\mathrm{P}_{5} 22$ were indexed with unit cell dimensions of $\mathrm{a}=\mathrm{b}=\sim 104 \AA, \mathrm{c}=\sim 148 \AA, \alpha=\beta=90^{\circ}, \gamma=120^{\circ}$. The hexagonal space group was confirmed by the absences of reflections along the screw axis $(0,0,6 n)$.

Structure determination was attempted using the SAD phasing programs hkl2map and Phenix AutoSol. The structure of the SCOC(78-159)-FEZ1(225-295) complex could not be determined using crystals from PEG 20.000 conditions because the anomalous signal derived from SAD datasets was too weak. Another approach using the structure of a SCOC dimer (PDB code 4BWD) as a model for molecular replacement failed as well.

\subsubsection{Optimization of SCOC(78-159)-FEZ1(225-295) crystals in isopropanol conditions}

Crystals obtained from commercial Protein Complex screen condition number 31 had a sharp triangular shape but were little (Fig. 3.3.6. D). Because crystals from PEG 20000 conditions could not be further improved these were the only crystals found in different conditions, an optimization approach was started for the isopropanol condition. Initial crystallization condition $20 \%$ (v/v) 2-Propanol, 0.1 M Tri-Sodium Citrate Dihydrate pH 5.6, $20 \%$ (w/v) PEG 4000 was reproduced with self-made crystallization solutions in 24-well hanging drop Linbro plates. Crystals started growing after 24 hours in conditions at various $\mathrm{pH}$ and isopropanol concentrations. Initial crystals from 24-well plates had an irregular shape and a very smeary diffraction pattern was observed at the synchrotron (Fig. 3.3.16. A-C).

Optimization included a 96-well Hampton Additive Screen that identified glycerol as a successful additive. The initial conditions were optimized to a final condition containing $20 \%$ (v/v) 2-Propanol, 0.1 M Tri-Sodium Citrate Dihydrate pH 4.6, $5 \%$ (w/v) PEG 4000, $10 \%$ (w/v) glycerol. Optimized crystals had sharp edges and a well-defined triangular shape (Fig. 3.3.26. D, E). Crystals were cryoprotected in reservoir solution supplied with additional $15 \%$ glycerol and flash cooled in liquid nitrogen. Crystals diffracted to a resolution of $2.3 \AA$ with well-defined spots (Fig. 3.3.24. F). 
To analyze the content of the crystals, whether they contained both SCOC and FEZ1 they were fished from the crystallization drops, washed in mother liquor and solubilized in gel filtration buffer before Schägger gel electrophoresis. Comparison with the purified complex revealed that both proteins crystallized in isopropanol conditions (Fig 3.3.27.).

A

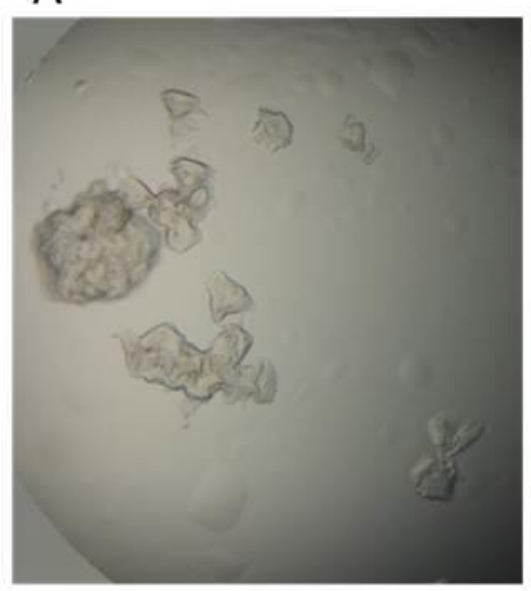

D

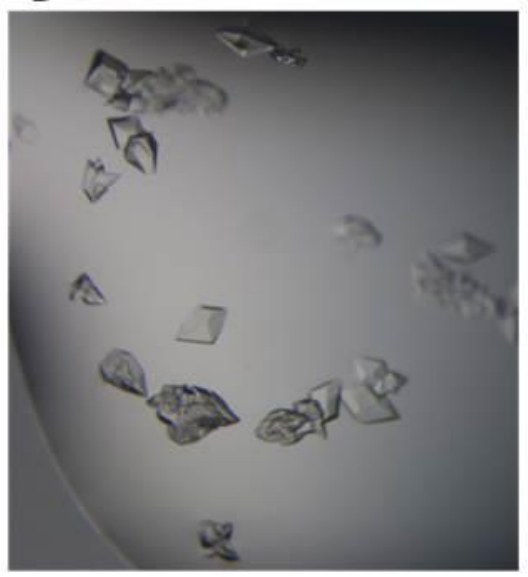

B

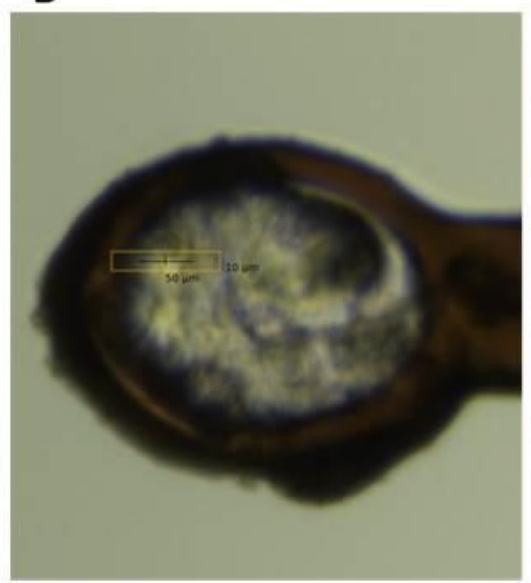

$\mathrm{E}$

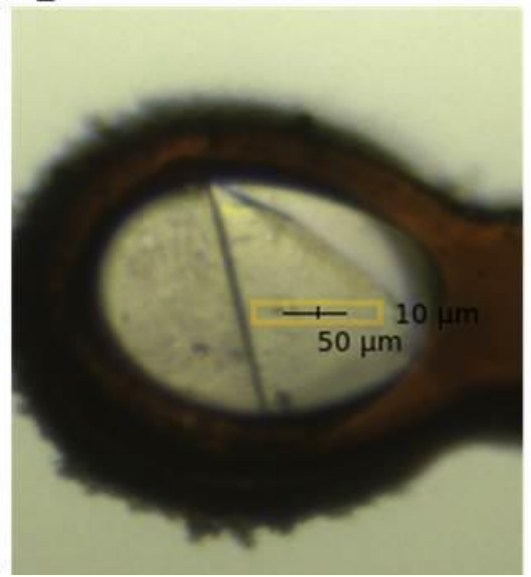

C

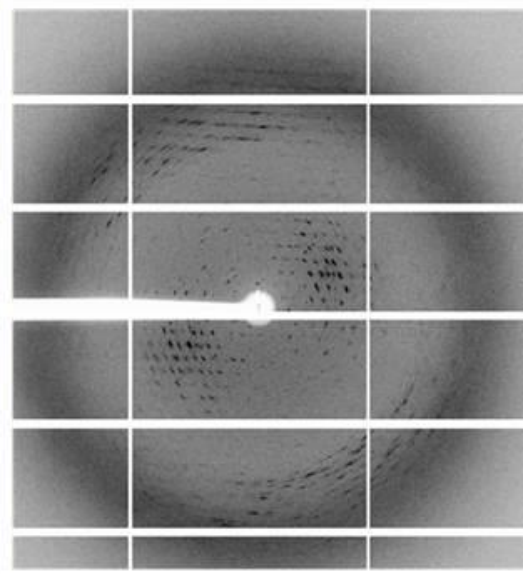

$\mathrm{F}$

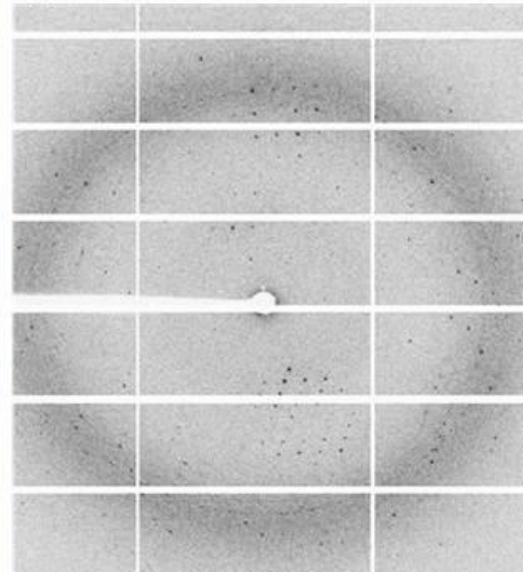

Figure 3.3.16.: SCOC(78-159)-FEZ1(225-295) crystals grown in isopropanol conditions at beamline X10SA (Swiss Light Source, Paul Scherrer Institute, Villigen, Switzerland). A: Initial crystals grown in 24-well Linbro plates, B: Initial crystal mounted at the beamline, C: Diffraction pattern observed from the same crystal (B). D: Optimized crystals grown in 24-well Linbro plates, E: Optimized crystal mounted at the beamline, F: Diffraction pattern observed from the same crystal (E). The yellow square has a size of $10 \times 50 \mu \mathrm{m}(\mathrm{B}, \mathrm{E})$. 


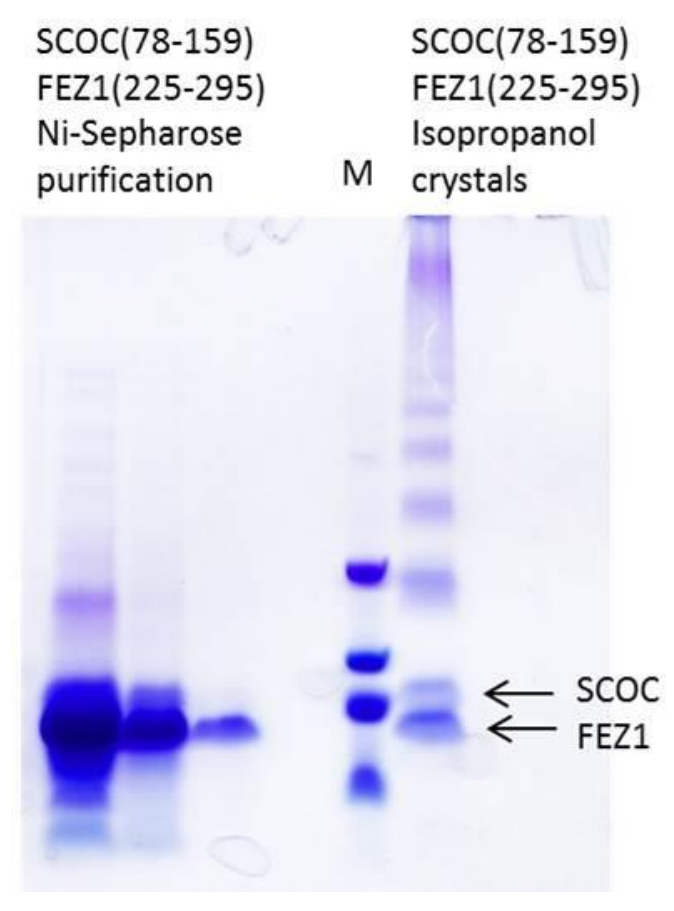

Figure 3.3.17.: Schägger gel electrophoresis of Ni-Sepharose purified SCOC(78-124)-FEZ1(225295) complex and isopropanol crystals. M: Marker. First three line shows the elution fractions of SCOC(78-124)-FEZ1(225-295) from Ni-Sepharose purification. Sixth line show washed and solubilized SCOC(78-124)-FEZ1(225-295) isopropanol crystals.

\subsubsection{X-ray data collection and analysis of SCOC(78-159)-FEZ1(225-295) crystals}

\section{from isopropanol conditions}

Data sets from native or selenomethionine labeled crystals were collected at $100 \mathrm{~K}$ at beamline X10SA (Swiss Light Source, Paul Scherrer Institute, Villigen, Switzerland). For selenomethionine labeled crystals a fluorescence spectrum was recorded to measure the selenium absorption spectrum (Fig. 3.3.18.). The wavelength was tuned to the selenium peak prior to data collection. Calculations by XDS revealed a strong anomalous signal for selenomethionine labeled crystals. Datasets were collected with a $0.1^{\circ}$ oscillation for $360^{\circ}$ at Se peak or $1 \AA$ wavelength with a maximum diffraction of $2.2 \AA$. 


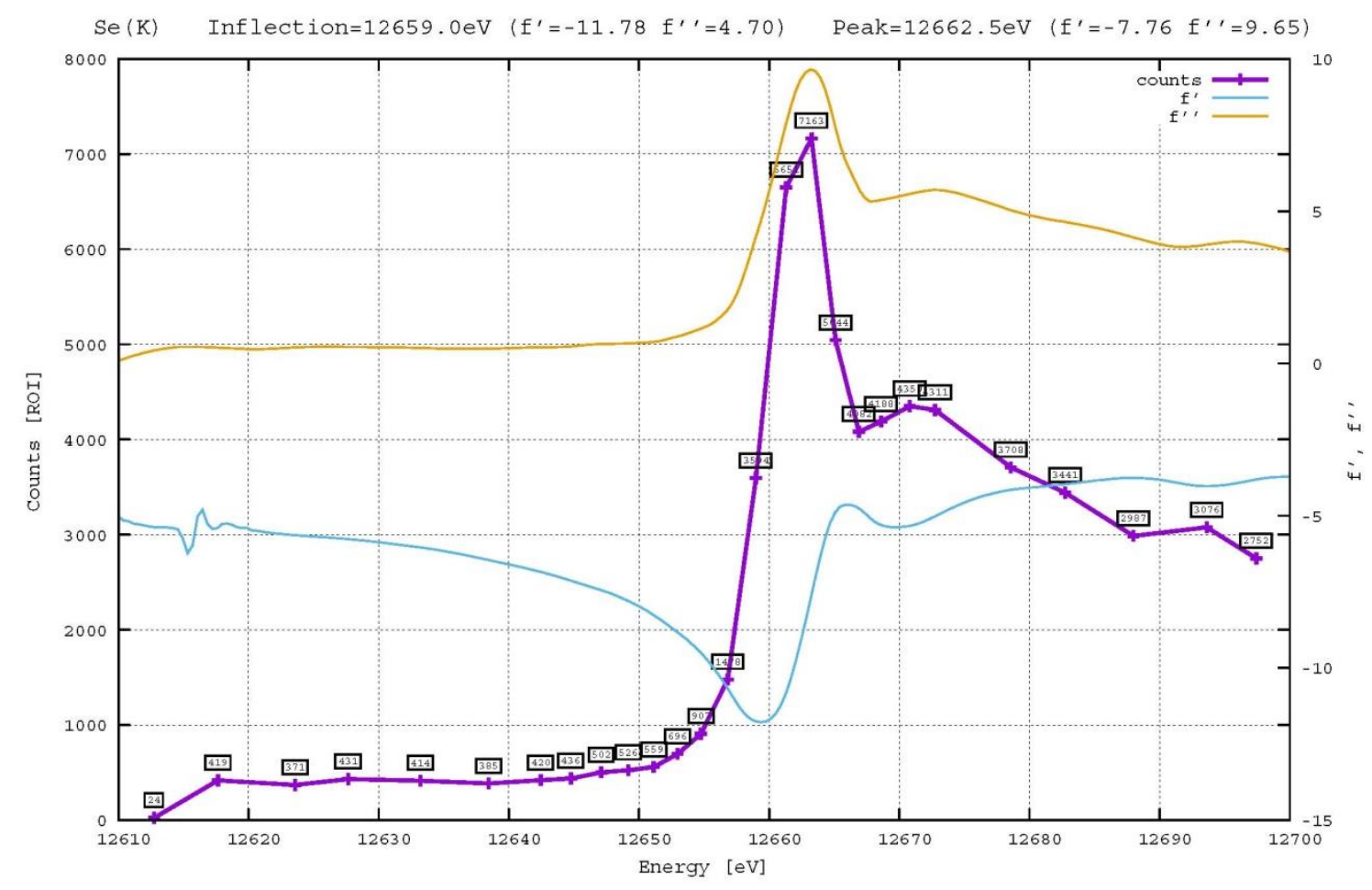

Figure 3.3.18.: Fluorescence spectrum of selenomethionine labeled SCOC(78-159)-FEZ1(225295) isopropanol crystals. Crystals grown in isopropanol conditions. Spectrum measured at beamline X10SA (Swiss Light Source, Paul Scherrer Institute, Villigen, Switzerland). Counts in the region of interest (ROI) are recorded as a function of energy (in eV). Blue curve: Counts (ROI), red curve: fitted $\mathrm{f}^{\prime}$, green curve: fitted: $\mathrm{f}^{\prime \prime}$.

Data were processed with input values specific for the PILATUS 6M detector using the XDS software package. An unambiguous space group could not be determined (Table 3.3.7.).

Table 3.3.7.: Determination of lattice character and bravais lattice from XDS IDXREF for SCOC-FEZ1 isopropanol crystals.

\begin{tabular}{|c|c|c|c|c|c|c|c|c|c|c|c|c|c|c|c|c|c|c|c|c|}
\hline LATTICE- & BRAVAIS- & QUALITY & UNIT CELL & CONST & ANTS $(A$ & ANGSTRO & $E M \& D$ & EGREES) & & REI & IV L & 10 & & RAN & SFOP & MA & IO & & & \\
\hline CHARACTER & LATTICE & OF FIT & a & b & c & alpha & beta & gamma & & & & & & & & & & & & \\
\hline * 44 & $\mathrm{aP}$ & $\theta . \theta$ & 59.4 & 67.2 & 67.2 & 116.5 & 90.0 & 90.0 & -1 & $\theta$ & $\theta$ & $\theta$ & $\theta$ & $\theta$ & -1 & $\theta$ & $\theta$ & -1 & $\theta$ & $\theta$ \\
\hline * 31 & $\mathrm{aP}$ & 0.2 & 59.4 & 67.2 & 67.2 & 63.5 & 90.0 & 90.0 & 1 & $\theta$ & $\theta$ & $\theta$ & $\theta$ & $\theta$ & 1 & $\theta$ & $\theta$ & -1 & $\theta$ & $\theta$ \\
\hline * 25 & $\mathrm{mC}$ & 0.3 & 70.7 & 114.3 & 59.4 & 90.0 & 90.1 & 90.0 & $\theta$ & -1 & -1 & $\theta$ & $\theta$ & -1 & 1 & $\theta$ & -1 & $\theta$ & $\theta$ & $\theta$ \\
\hline * 35 & $\mathrm{mP}$ & 0.6 & 67.2 & 59.4 & 67.2 & 90.0 & 116.5 & 90.0 & $\theta$ & $\theta$ & 1 & $\theta$ & 1 & $\theta$ & $\theta$ & $\theta$ & $\theta$ & 1 & $\theta$ & $\theta$ \\
\hline * 23 & $\mathrm{OC}$ & 0.8 & 70.7 & 114.3 & 59.4 & 90.0 & 90.1 & 90.0 & $\theta$ & -1 & -1 & $\theta$ & $\theta$ & -1 & 1 & $\theta$ & -1 & $\theta$ & $\theta$ & $\theta$ \\
\hline * 20 & $\mathrm{mC}$ & 1.1 & 114.3 & 70.7 & 59.4 & 89.9 & 90.0 & 90.0 & $\theta$ & -1 & 1 & $\theta$ & $\theta$ & 1 & 1 & $\theta$ & -1 & $\theta$ & $\theta$ & $\theta$ \\
\hline 41 & $\mathrm{mC}$ & 34.4 & 120.4 & 67.2 & 59.4 & 90.0 & 90.1 & 86.6 & $\theta$ & 2 & 1 & $\theta$ & $\theta$ & $\theta$ & 1 & $\theta$ & 1 & $\theta$ & $\theta$ & $\theta$ \\
\hline 40 & $O C$ & 34.8 & 67.2 & 120.4 & 59.4 & 89.9 & 90.0 & 93.4 & $\theta$ & $\theta$ & 1 & $\theta$ & $\theta$ & -2 & -1 & $\theta$ & 1 & $\theta$ & $\theta$ & $\theta$ \\
\hline 30 & $\mathrm{mC}$ & 34.9 & 67.2 & 120.4 & 59.4 & 89.9 & 90.0 & 86.6 & $\theta$ & $\theta$ & 1 & $\theta$ & $\theta$ & 2 & 1 & $\theta$ & -1 & $\theta$ & $\theta$ & $\theta$ \\
\hline 22 & $\mathrm{hP}$ & 35.0 & 67.2 & 67.2 & 59.4 & 90.0 & 90.0 & 116.5 & $\theta$ & $\theta$ & -1 & $\theta$ & $\theta$ & -1 & $\theta$ & $\theta$ & -1 & $\theta$ & $\theta$ & $\theta$ \\
\hline 37 & $\mathrm{mC}$ & 249.9 & 146.9 & 59.4 & 67.2 & 90.0 & 114.1 & 66.2 & -1 & -2 & $\theta$ & $\theta$ & -1 & $\theta$ & $\theta$ & $\theta$ & $\theta$ & $\theta$ & -1 & $\theta$ \\
\hline 39 & $\mathrm{mC}$ & 250.1 & 146.9 & 59.4 & 67.2 & 90.0 & 114.1 & 66.2 & -1 & -2 & $\theta$ & $\theta$ & -1 & $\theta$ & $\theta$ & $\theta$ & $\theta$ & $\theta$ & -1 & $\theta$ \\
\hline 42 & oI & 284.1 & 59.4 & 67.2 & 134.2 & 93.1 & 116.2 & 90.0 & 1 & $\theta$ & $\theta$ & $\theta$ & $\theta$ & $\theta$ & 1 & $\theta$ & -1 & -2 & -1 & $\theta$ \\
\hline 33 & $\mathrm{mP}$ & 286.0 & 59.4 & 67.2 & 67.2 & 116.5 & 90.0 & 90.0 & -1 & $\theta$ & $\theta$ & $\theta$ & $\theta$ & $\theta$ & -1 & $\theta$ & $\theta$ & -1 & $\theta$ & $\theta$ \\
\hline
\end{tabular}


Phenix Xtriage analysis revealed that SCOC(78-159)-FEZ1(225-295) crystals from isopropanol conditions are twinned (Fig. 3.3.19. and 3.3.20.). Two pseudo-merohedral twin operators were found. Precise determination of the space group and cell dimensions failed as well as structure determination using the SAD phasing programs hkl2map and Phenix AutoSol.

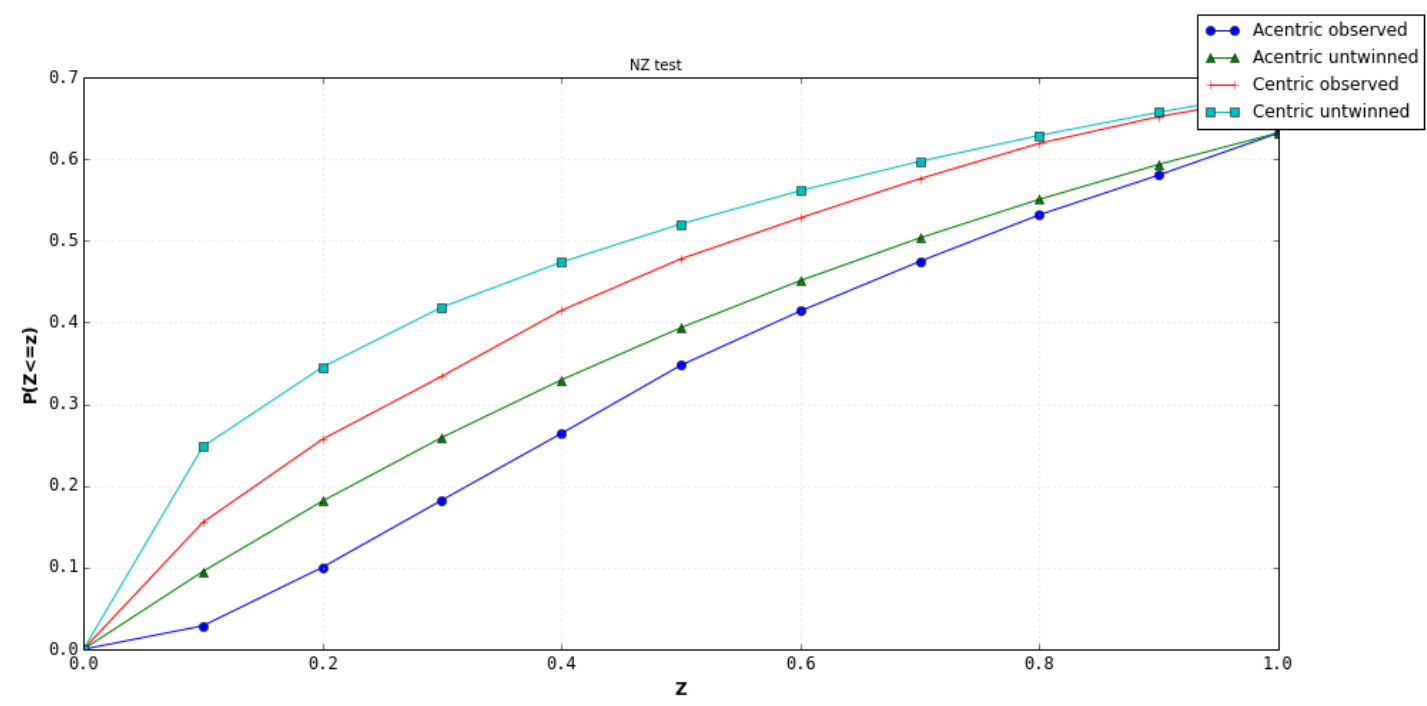

Figure 3.3.19.: Britton analyses from Phenix Xtriage for SCOC-FEZ1 isopropanol crystals

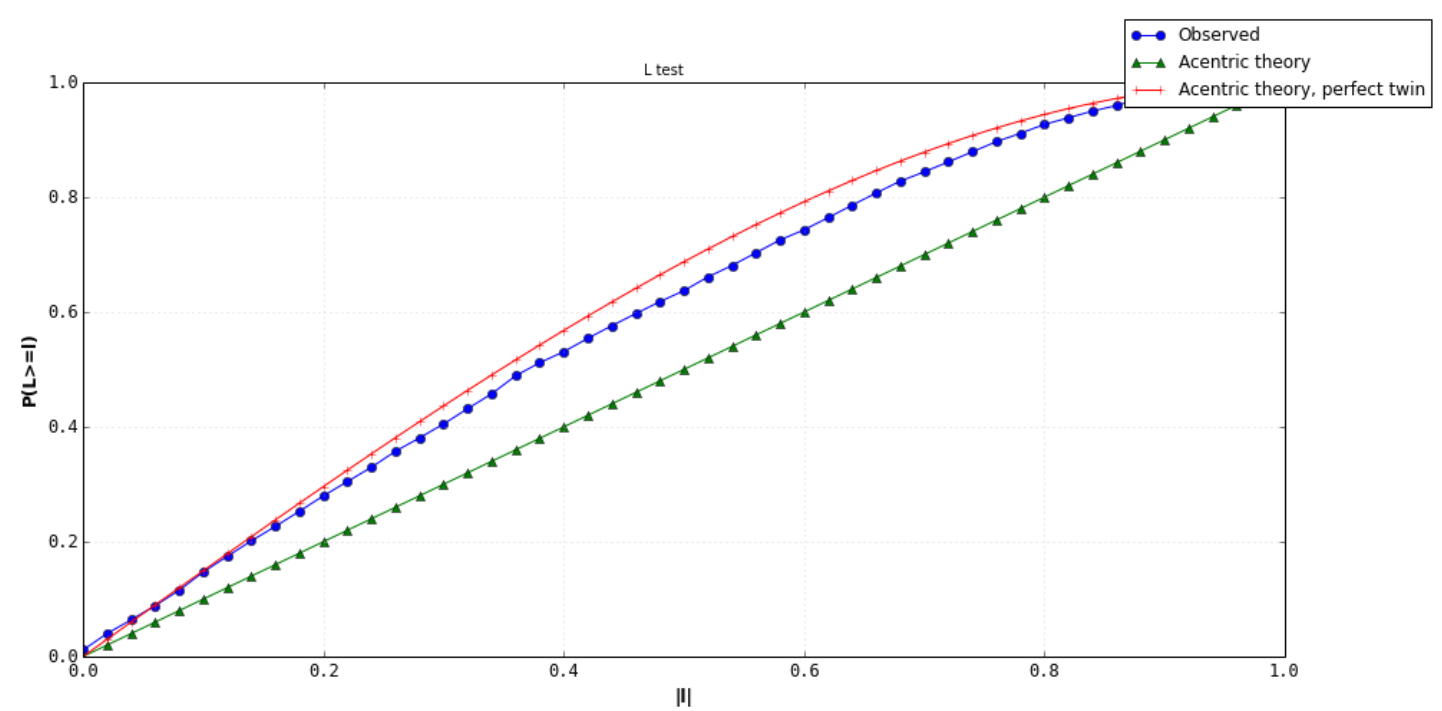

Figure 3.3.20: $\mathrm{L}$ test for acentric data from Phenix Xtriage for SCOC-FEZ1 isopropanol crystals 


\subsubsection{Cross-linking of the SCOC-FEZ1 complex and analysis by mass spectrometry}

In order to gain more insight in the SCOC-FEZ1 complex architecture, chemical cross-linking of the complex and analysis by mass spectrometry were conducted. Therefore the SCOC(78159)-FEZ1(225-295) complex was cross-linked with the amine-reactive bis(sulfosuccinimidyl) suberate (BS3) cross-linker at different concentrations. The amount of cross-links was analyzed by native gel electrophoresis (Fig. 3.3.21.). Without cross-linker only the two bands for SCOC and FEZ1 below $15 \mathrm{kDa}$ were observed. In contrast after addition of BS3 more bands with a higher molecular weight appeared. The number of these bands enriched with increasing cross-linker concentration. Two bands, marked with black arrows (Fig. 3.3.21.) were sent for mass spectrometry analysis. This work was conducted in collaboration with Dr. Momchil Ninov (Department of Neurobiology) and Dr. Chung-Tien Lee (Bioanalytical Mass Spectrometry).

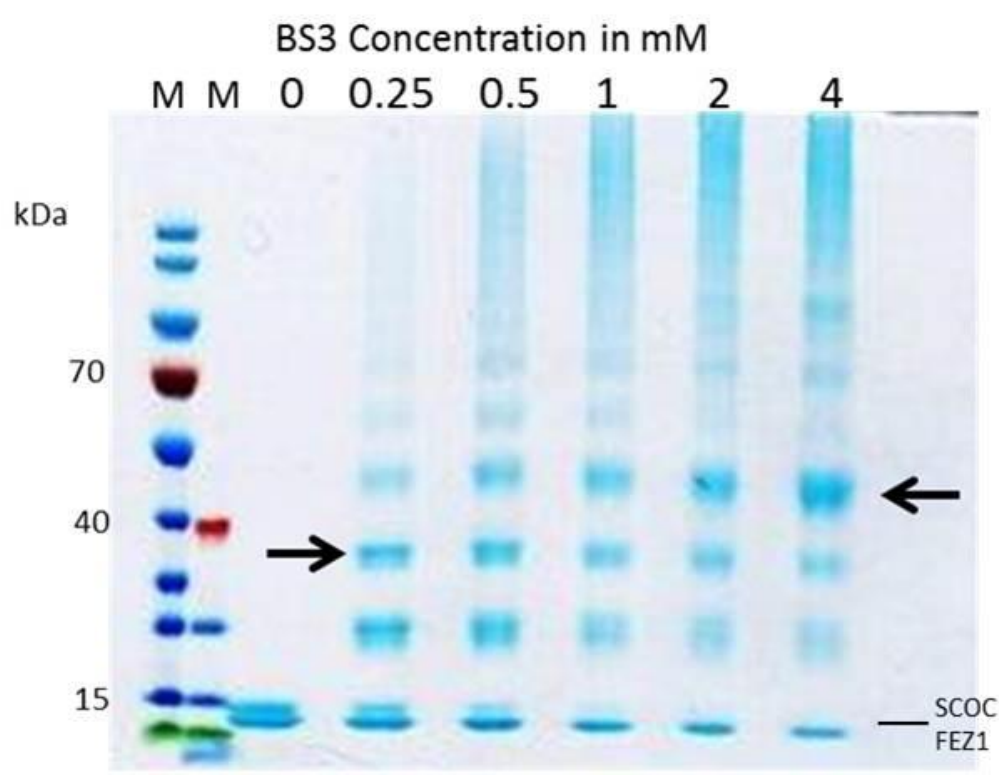

Figure 3.3.21.: Cross-linking of the SCOC(78-159)-FEZ1(225-295) complex. Native gel electrophoresis of the SCOC-FEZ1 complex ( $5 \mathrm{mg} / \mathrm{ml})$, cross-linked with $0,0.25,0.5,1,2$ and $4 \mathrm{mM}$ BS3. Arrows mark bands that were analyzed by mass spectrometry. Left arrow flags band1, right arrow band2. M: Marker.

Bands were cut from the gel, digested with trypsin and sent to LC-MS/MS analysis. Each sample was analyzed in two technical replicates. Raw data were searched against a FASTA database containing the full length sequences of human SCOC and FEZ1 using the targetdecoy strategy of pLink. Results are shown with the count of spectrum and the maximum score for each cross-link (Table 3.3.8.). Inter- and intramolecular cross-links were visualized 
with xiNET [26] (Fig. 3.3.22.). To exclude false positive cross-links the threshold for the maximal score was set to 5.1 .
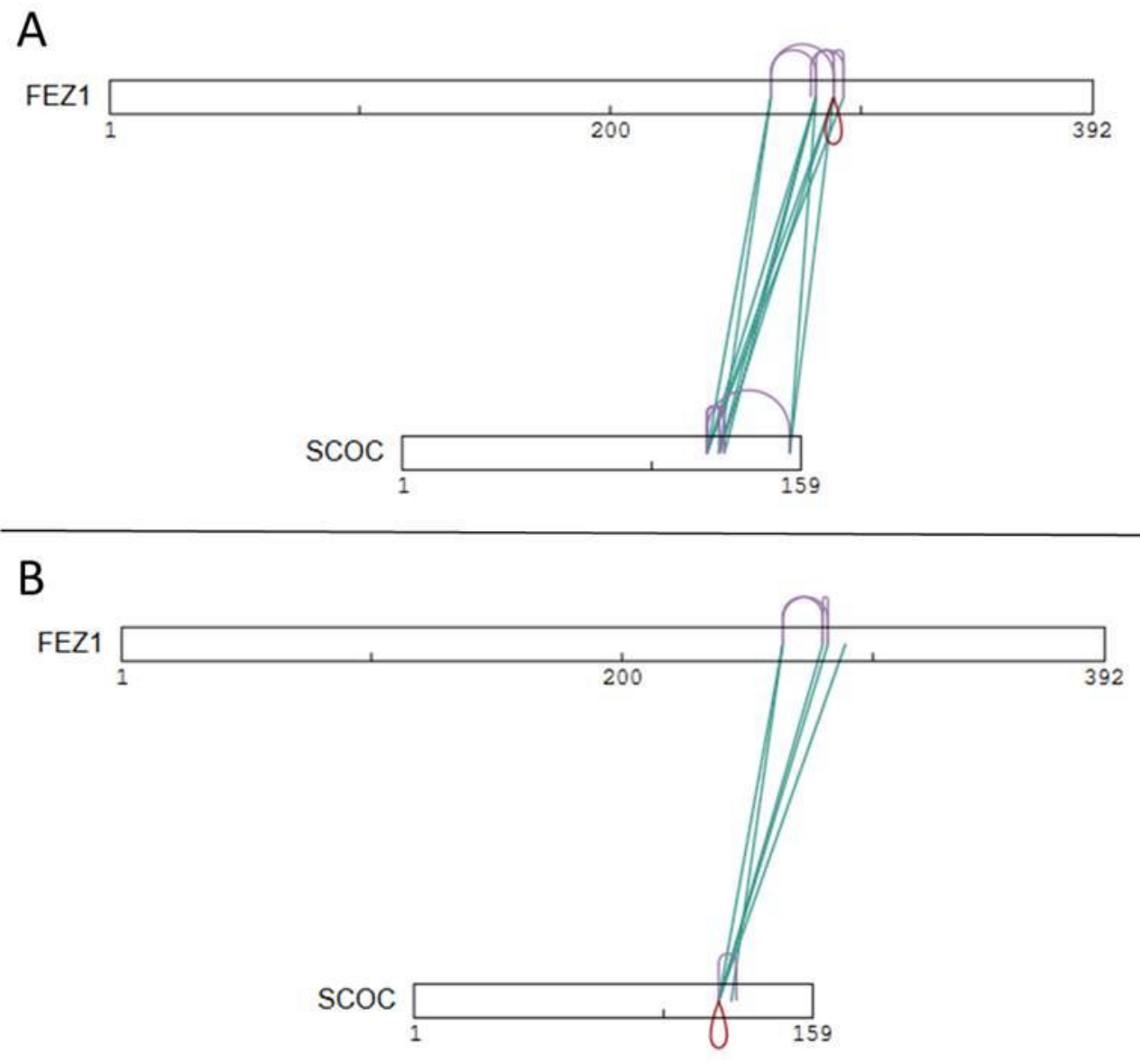

Figure 3.3.22.: Visualization of the mass spectrometry analysis of the cross-linking of the SCOC(78-159)-FEZ1(225-295) complex. A: shows analysis of band1, B: shows analysis of band2 from (Fig 3.3.21.) Samples were run in two technical replicates. Threshold was set to 5.1. Turquoise lines inter cross-links between SCOC and FEZ1. Purple lines inter- or intra molecular cross-links, Red line: Inter-molecular self-link. Figures made with xiNET. 
Table 3.3.8.: Data of the mass spectrometry analysis of the SCOC(78-159)-FEZ1(225-295) complex cross-linking. Samples were run in two technical replicates. Threshold was set to 5.1.

\begin{tabular}{|c|c|c|c|c|c|c|c|}
\hline \multirow[b]{2}{*}{ Protein1 } & \multirow[b]{2}{*}{ Residue1 } & \multirow[b]{2}{*}{ Protein2 } & \multirow[b]{2}{*}{ Residue2 } & \multicolumn{2}{|c|}{ Band1 } & \multicolumn{2}{|c|}{ Band2 } \\
\hline & & & & $\begin{array}{l}\text { Count of } \\
\text { spectrum }\end{array}$ & $\begin{array}{c}\text { Max of } \\
\text { score }\end{array}$ & $\begin{array}{l}\text { Count of } \\
\text { spectrum }\end{array}$ & $\begin{array}{c}\text { Max of } \\
\text { score }\end{array}$ \\
\hline FEZ1 & 264 & SCOC & 122 & 1 & 11.38 & 4 & 14.53 \\
\hline & 264 & & 127 & 10 & 16.74 & 4 & 12.84 \\
\hline & 280 & & 122 & & & 3 & 12.27 \\
\hline & 282 & & 122 & 4 & 9.25 & 5 & 8.83 \\
\hline & 282 & & 127 & 1 & 7.2 & & \\
\hline & 282 & & 129 & 3 & 5.71 & & \\
\hline & 282 & & 155 & 8 & 8.68 & & \\
\hline & 289 & & 122 & 4 & 6.6 & 1 & 8.63 \\
\hline & 289 & & 127 & 4 & 7.33 & & \\
\hline & 289 & & 155 & 6 & 5.6 & & \\
\hline & 293 & & 122 & 1 & 5.38 & & \\
\hline FEZ1 & 264 & $\overline{\text { FEZ1 }}$ & 280 & & & 2 & 10.88 \\
\hline & 264 & & 282 & 2 & 6.71 & 2 & 7.49 \\
\hline & 264 & & 289 & 1 & 5.3 & & \\
\hline & 280 & & 282 & & & 2 & 8.82 \\
\hline & 280 & & 293 & 1 & 5.13 & & \\
\hline & 282 & & 289 & 2 & 8.29 & & \\
\hline & 289 & & 289 & 1 & 5.25 & & \\
\hline & 289 & & 293 & 3 & 5.52 & & \\
\hline SCOC & 122 & SCOC & 155 & 24 & 14.28 & & \\
\hline & 122 & & 127 & 7 & 14.56 & & \\
\hline & 122 & & 129 & 1 & 10.89 & 5 & 10.22 \\
\hline & 122 & & 122 & & & 1 & 8.93 \\
\hline & 127 & & 129 & 1 & 7.52 & & \\
\hline
\end{tabular}

The cross-linked lysine residues were mapped onto the SCOC structure (PDB code: 4BWD) and $\mathrm{C} \alpha-\mathrm{C} \alpha$ distances were measured using PyMol. To evaluate the intramolecular cross-links found for FEZ1 a coiled coil model was prepared using CCBuilder V1.0 [145] for either a parallel or antiparallel coiled coil dimer formed of residues 225-295. Intramolecular crosslinks of SCOC are in good agreement with the known structure of the parallel coiled coil SCOC dimer (Fig. 3.3.20.). This suggests that BS3 cross-links one chain of the dimer to the other subunit. Due to the fact that all lysine residues are in close proximity to each other, only short range cross-links in allowed distance were found. The most prominent cross-link of K122 to K155 is not shown because the SCOC structure includes only residues 86 to 146. 


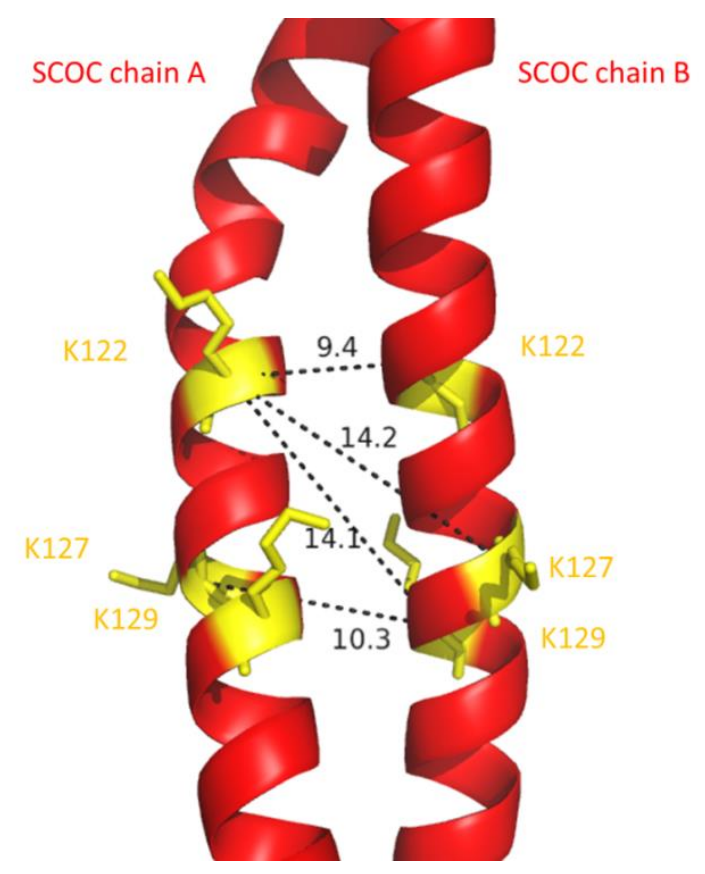

Figure 3.3.23.: Intra molecular cross-links of SCOC. Cartoon representation of SCOC in red (PDB code 4BWD). Lysines are presented as sticks in yellow. $\mathrm{C} \alpha-\mathrm{C} \alpha$ distances $(\AA)$ between intra molecular cross-links are shown as dashed lines.

Intramolecular cross-links for FEZ1 were mapped onto models of either a parallel or antiparallel coiled coil dimer. Intramolecular cross-link distances still lay in a possible range for the parallel coiled coil model (Fig. 3.3.21.). In the antiparallel coiled coil model, cross-link distances are too long and exceeded the distance restrains of the cross-linker (Fig. 3.3.22.). Especially the cross-link between K264 and K289 has a very long distance of $50 \AA$, when mapped between the two chains. However, a short intramolecular cross link on the same chain is possible as well. The cross-link between K282 and K289 is also either short (10 ̊) when mapped on the same chain or very long (77 $\AA$ ) between the two chains (Fig. 3.3.22.) taking into account the cross-linker arm length. Considering an anti-parallel coiled coil a cross-link between K264 and K264 on the other chain is expected but was not found. Intramolecular cross-links of FEZ1 strongly suggest that FEZ1 forms a parallel coiled coil dimer. 
A Fez1 model

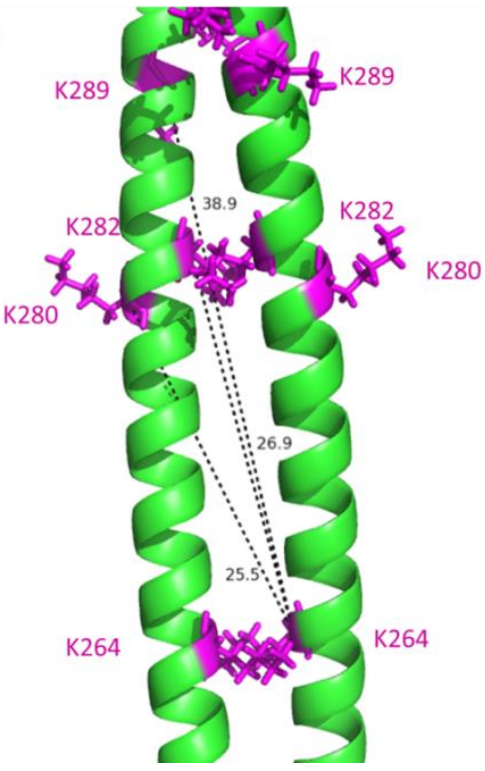

B

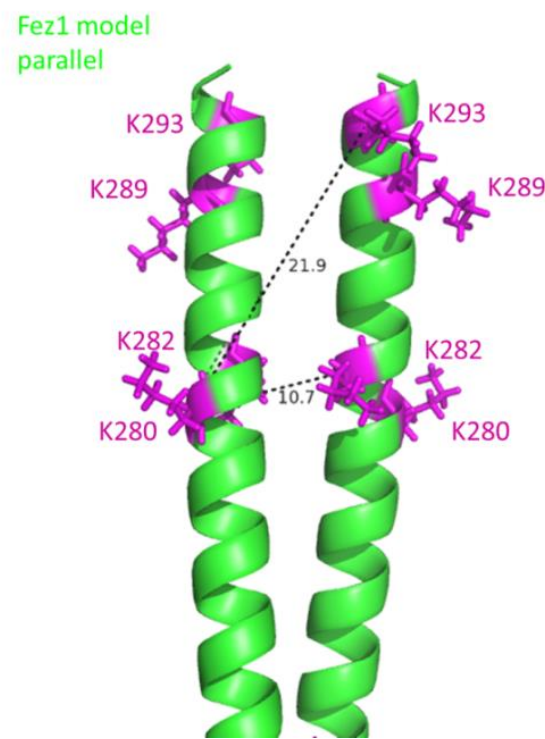

Figure 3.3.24.: Intra molecular cross-links of parallel FEZ1 model. Cartoon representation of parallel FEZ1 coiled coil dimer model (made with CCBuilder) in green. Lysines are presented as sticks in magenta. $\mathrm{C} \alpha-\mathrm{C} \alpha$ distances $(\AA)$ between intra molecular cross-links are shown as dashed lines.

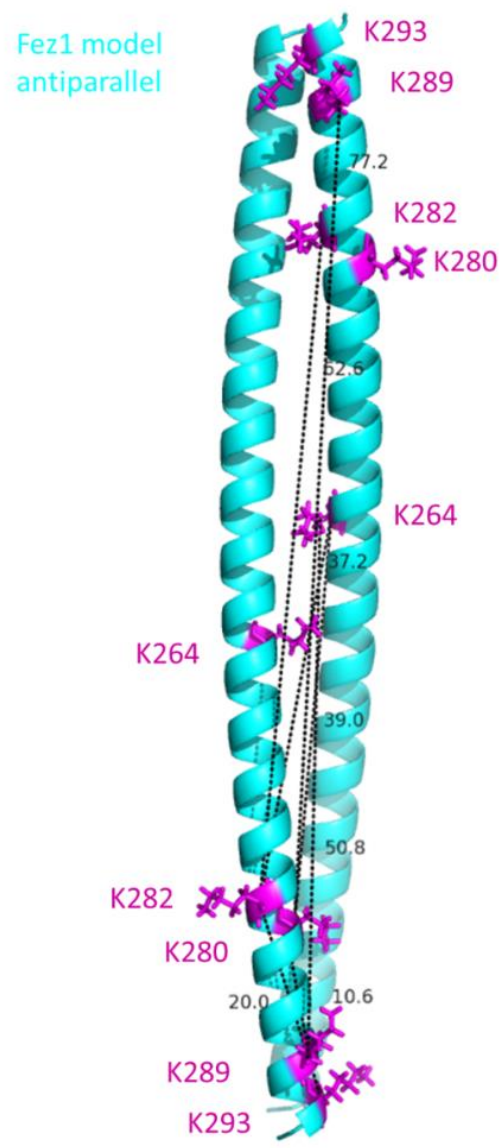

Figure 3.3.25.: Intra molecular cross-links of antiparallel FEZ1 model. Cartoon representation of antiparallel FEZ1 coiled coil dimer model (made with CCBuilder) in turquoise. Lysines are presented as sticks in magenta. $\mathrm{C} \alpha-\mathrm{C} \alpha$ distances $(\AA)$ between intra molecular cross-links are shown as dashed lines. 
The maximum of score for intra molecular cross-links is similar for both SCOC and FEZ1, the maximum score of inter molecular cross-links between SCOC and FEZ1 is higher. Assuming a stable complex formation where lysine residues come in a distance favorable for cross-linking. To analyze the possible architecture of the SCOC-FEZ1 complex two models were visualized assuming that FEZ1 forms a parallel dimer (Fig. 3.3.23.). One model represents antiparallel binding of the two parallel coiled coil dimers (Fig. 3.3.23. A), whereas the other shows parallel binding of the two dimers (Fig. 3.3.23. B). C $\alpha$-C $\alpha$ distances of inter molecular cross-links were mapped but not measured.

Cross-links between FEZ1 residues K280, K282 and K289 and SCOC residues K122, K127 and K129 are close and in a possible range for both models. In contrast, in the antiparallel model inter molecular cross-links with highest scores between SCOC residue K155 to FEZ1 K282 and K289 and between FEZ1 K264 and SCOC K122 and K127 lay in a long distance and intersect with each other (Fig. 3.3.23. A). Cross-links between SCOC K155 and FEZ1 K264 are more likely to be expected for an antiparallel complex model. The same cross-links are seen in a more likely distance for the parallel model (Fig. 3.3.23. B). Therefore, I propose a model of two parallel coiled coil dimers forming a parallel SCOC-FEZ1 complex. 
A

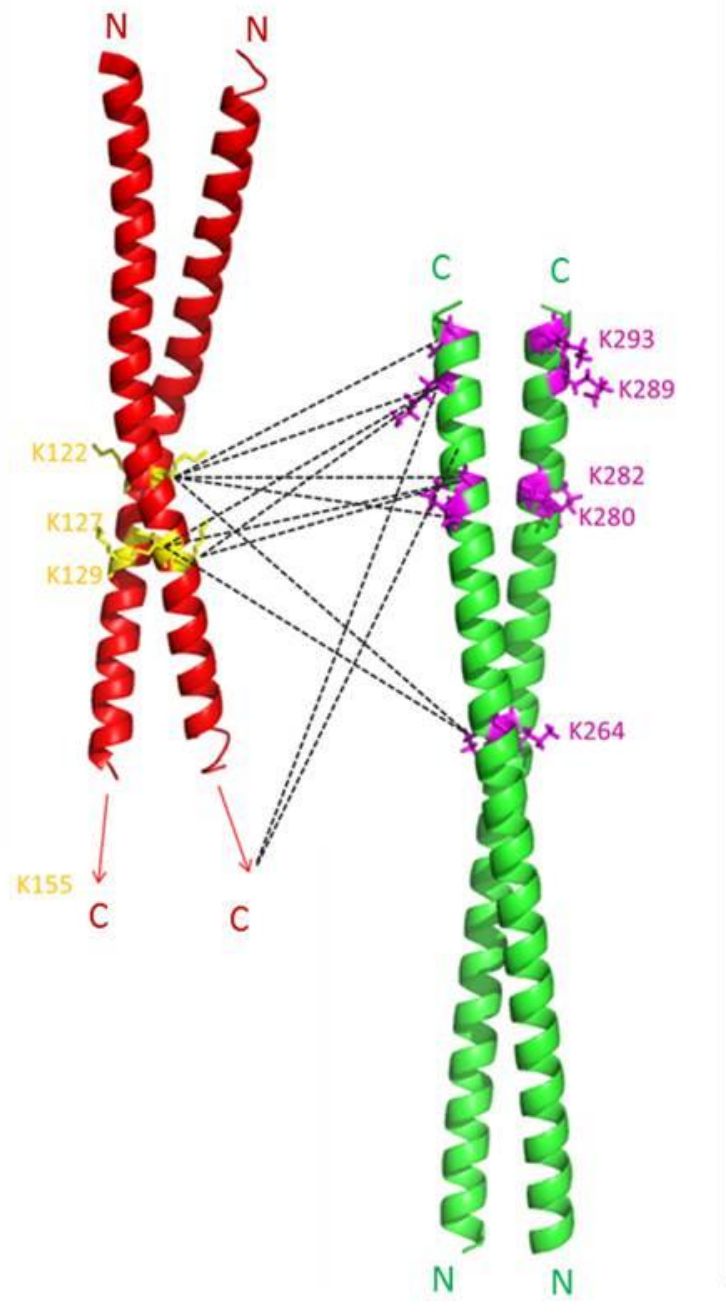

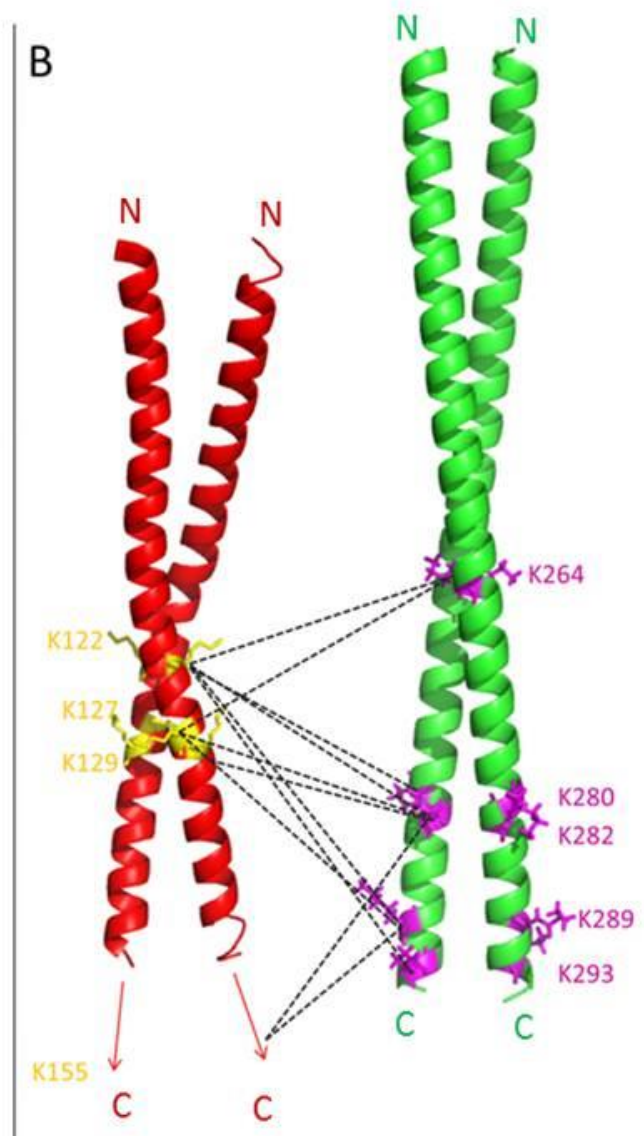

Figure 3.3.26.: Inter molecular cross-links of SCOC and a parallel coiled coil FEZ1 model. Cartoon representation of SCOC in red (PDB code: 4BWD) and parallel coiled coil FEZ1 dimer model (made with CCBuilder) in green. A: Antiparallel binding model. B: Parallel binding model. Lysines are presented as sticks in yellow for SCOC and magenta for FEZ1. C $\alpha$-C $\alpha$ distances ( $\AA$ ) between intra molecular cross-links are shown as dashed lines. 


\section{Discussion}

\subsection{Two Rab33B molecules form a complex with the diverging C-termini of an Atg16L1 dimer}

During autophagy a double layered isolation membrane forms de novo that expands and encloses its cargo. The membrane supply of the growing isolation membrane involves various sources at different stages and is still under discussion [74]. In 2008 Itoh et al. first described the direct interaction between a small Rab GTPase and an Atg protein [54]. They demonstrated complex formation of Rab33B with the coiled coil domain of Atg16L1 in a GTP-dependent manner. In more detail Rab33B forms a complex with the $\operatorname{Atg} 12 \sim \operatorname{Atg} 5 / \operatorname{Atg} 16 \mathrm{~L} 1$ complex via direct interaction with the Atg16L1 coiled coil domain. It was proposed that Rab33B-Atg16L1 interaction may facilitate the recruitment of vesicles originating from the Golgi to the growing isolation membrane [54].

Insights into the molecular details of Rab33-Atg16L1 interaction were still lacking, which would help to understand the function of this complex. In this study I present the X-ray crystal structure of the mRab33B(30-202)Q92L-mAtg16L1(153-210) complex at $3.47 \AA$ resolution. It reveals that two Rab33B molecules form a complex with the diverging C-terminus of an Atg16L1 dimer respectively. A 1:1 stoichiometry of the complex was proposed before by Dr. Amanda Schalk in our laboratory and was verified by the structure. The Rab33B binding site for Atg16L1 is typical for binding of effector proteins and explains the GTP dependency of the interaction.

Protein cross-linking experiments done in solution support the architecture of the Rab33BAtg16L1 complex found in the crystal structure. Lysines that are in proximity in the crystal structure were cross-linked with high scores. Several lysines that formed inter-molecular cross-links lay in a distance of $40 \AA$ between $\mathrm{C} \alpha$ atoms. These values are in the range of values observed in other structures [87]. Cross-linking was done in solution where lysine side chains are often flexible and proteins are dynamic and cross-linking can lock proteins in a closer position than they normally adopt.

In this study I found that the minimal Rab33B binding site of Atg16L1 comprises residues 163-210. Previous studies in our group showed that a truncation to residue 204 of the Atg16L1 coiled coil domain disrupted complex formation, while constructs extending to 210 still formed a complex with Rab33B. The crystal structure revealed that Rab33B-Atg16L1 interaction takes place at the very C-terminal end of the Atg16L1 coiled coil domain within 
residues 191 to 208. This confirms the proposal that the Atg16L1 site of homooligomerization (80-200) and Rab33B binding site (141-265) are spatially separated [54]. Interestingly, the $\mathrm{N}$-terminal part is also important for complex formation because a deletion of residues 163-172 disrupted complex formation even when the construct extended to residue 210. I observed that the mRab33B(30-202)Q92L-mAtg16L1(153-210) complex was much more stable than the complex with the minimal binding site mRab33B(30-202)Q92LmAtg16L1(163-210), which dissociated during purification.

Bachelor student Johannes Groffmann analyzed the Rab33B-Atg16 interactions with NiSepharose pulldown experiments under my supervision. Co-expressed mRab33B(30202)Q92L-Atg16L1 samples were purified with Ni-Sepharose beads. Eluted samples were then analyzed with Schägger gels and western blots and showed complex formation for mRab33B(30-202)Q92L with mAtg16L1 (125-234), (153-210) and (163-210). No complex formation was observed for mRab33B(30-202)Q92L with mAtg16L1 (172-210) and (153200) (Fig. 4.1.).

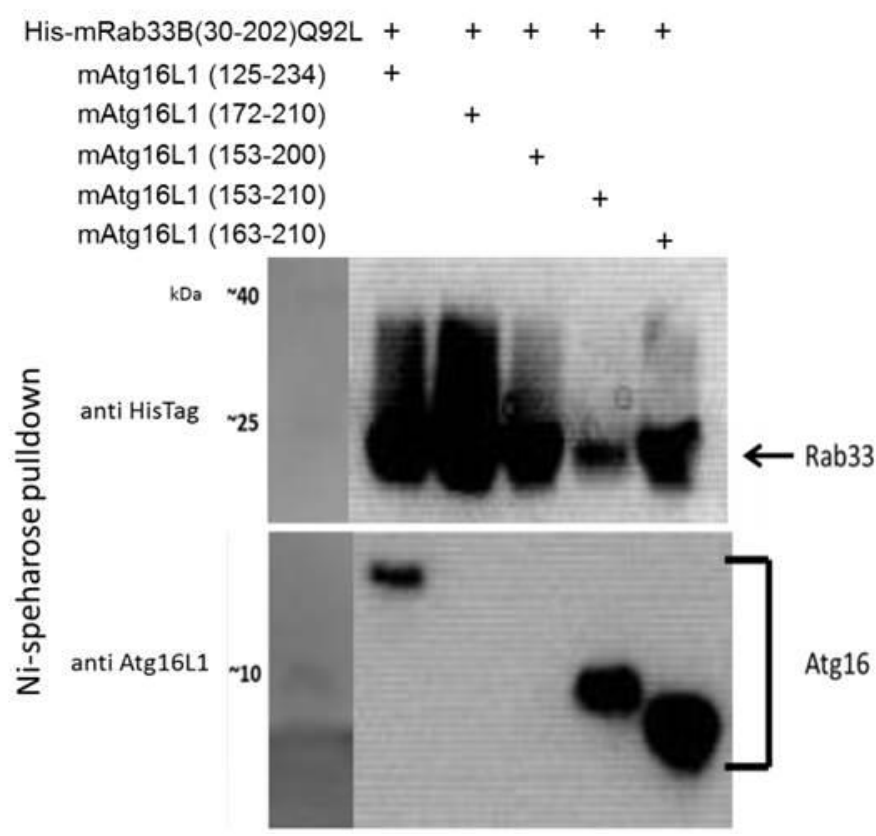

Figure 4.1.: Ni-Sepharose pull down of His-Rab33B(30-202)Q92L co-expressed with different Atg16L1 constructs. Experiment was done by bachelor student Johannes Groffmann.

The crystal structure shows that the N-terminal part of Atg16L1 mediates dimerization of the complex and a truncation at the N-terminal domain beyond residue 153 might destabilize the coiled coil formation. In contrast, at the C-terminus of the Atg16L1 coiled coil domain helices diverge from residue 189 onwards, which is the site (191-208) of complex formation with 
Rab33B. Studies on human Atg16L1 that shares $94 \%$ identity with murine Atg16L1 confirmed a stable coiled coil domain for residues 126-207 [107].

No structure is known for the mammalian Atg16 coiled coil alone, so we do not know whether complex formation with Rab33B affects the conformation of the Atg16L1 coiled coil C-termini. However, divergence of the helices at the $\mathrm{C}$-terminal end of the coiled coil domain is favored by the mainly charged residues at the a/d heptad position in this region (E201, R204 and E208). Coiled coil domains are stabilized by hydrophobic core packing with the typical repeating pattern "hxxhcxc". Polar residues at heptad position a or $\mathrm{d}$ have a destabilizing effect on the coiled coil packing [50]. Until now this feature of the Atg16L1 dimer was not described before.

The physiological role of the diverging C-termini remains unassigned. All mammalian homologs of Atg16L1 share a WD40 repeat domain at the C-terminus, other than yeast Atg16. The WD40 repeat domain is predicted to fold as a seven bladed $\beta$-propeller most probably providing a platform for protein complex assembly as reported for other WD40 repeat proteins [126]. Until now the function of the Atg16L1 WD40 repeat is unknown. The diverging C-termini may affect the orientation of the two WD40 repeats and thus may also control potential interactions with either membranes or other not yet identified interaction partners. The WD40 domain could help to localize Atg16L1 to membranes but Atg16L1 alone is not able to associate with membranes, expecting the requirement of other proteins [90]. To validate this hypothesis further investigations are required, like identification of mammalian autophagy specific proteins as interaction partners for the Atg16L1 WD repeat.

Atg16L1 binds to switch I and II of Rab33B. These regions are important for nucleotide binding and their conformations depend on whether GTP or GDP is bound, supporting the finding that complex formation is GTP-dependent [54]. I further investigated nucleotide dependent Rab33B binding of Atg16L1 in vitro and in vivo. The dominant negative mutant Rab33B(30-202)T47N that binds GDP did not bind Atg16L1(153-210) in in vitro pull down assays. Moreover, also in vivo co-immunoprecipitation experiments showed that Atg16L1 bound Rab33B T47N in significant lower amounts compared to GTP-bound Rab33B Q92L. Interestingly wild type Rab33B bound Atg16L1 in same amounts as Rab33B Q92L both in vitro and in vivo indicating that Rab33B WT is mainly present in the GTP bound state.

Since the structure was determined at low resolution (3.47 $\AA$ ) mutagenesis studies were done to verify the Rab33B-Atg16L1 interactions observed in the crystal structure. The following single site point mutants were prepared: F70A, F70E and W87A for Rab33B Q92L and 
K198A, A202W, N206K for Atg16L1. The in vitro pulldown assays showed that all point mutations abolished complex formation thus verifying the Rab33B-Atg16L1 crystal structure. When these mutations were introduced in the full-length proteins and co-overexpressed in HEK cells, for Rab33B Q92L mutants F70A and W87A and Atg16L1 K198A, A202W and N206K complex formation was nearly entirely abolished. For Rab33B Q92L/F70E a significantly reduced binding to Atg16L1 was observed. While the results of the in vitro and in vivo experiments are overall in good agreement, the small differences might be due to additional interaction partners being present in cells and variations in protein and salt concentrations. The conserved residues Rab33B F70 and W87, which I showed to be essential for complex formation with $\operatorname{Atg} 16 \mathrm{~L} 1$, are also important for complex formation of Rab9A with its effector RUTBC2 [148] and Rab5 mutants F57A and W74A disrupted complex formation with Rabaptin5 [151].

Fluorescence microscopy of cells co-overexpressing Rab33B and Atg16L1 were done to study the localization of the proteins and the complex. In cells overexpressing Atg16L1 and either Rab33B Q92L or wild-type Rab33B both proteins co-localized in punctate structures in the cytoplasm. Co-expression of GTP-bound Rab33B Q92L with Atg16L1 leads to enlarged punctate structures. In contrast, these punctate structures were dramatically decreased for the Rab33B T47N mutant and the single-site Rab33B Q92L and Atg16L1 mutants, which also abolished complex formation in vitro. Furthermore the Rab33B T47N mutant localized mostly to the perinuclear area, while Rab33B and Atg16L1 are evenly distributed in the cytosol upon inhibition of complex formation. The results of co-localization studies are in agreement with in vitro and in vivo co-immunoprecipitations.

Atg16L1 positive punctate structures were shown to co-localize with the pre-autophagosomal marker protein LC3 [111]. Rab33B may be essential for Atg16L1 recruitment to the preautophagosomal membrane. Since co-localization was studied under nutrient-rich conditions further experiments under starvation conditions and staining with LC3 or a Golgi specific marker need to be done to proof the identity of the punctuate structures. Furthermore GTPbound Rab33B Q92L increased LC3-II levels in co-immunoprecipitation experiments and the lipidation assay and enlarged punctuate structures were observed for cells overexpressing Atg16L1 with Rab33B Q92L. Results propose an effect of Rab33B Q92L on basal autophagy in nutrient rich conditions independent of complex formation with Atg16L1. Increased LC3-II levels regardless of nutrient conditions were observed before by Itoh et al. when Rab33B Q92L was overexpressed [54]. Rab33B may promote LC3 lipidation to recruit the Atg5 Atg12/Atg16 complex at specific sites amongst others. To further understand the role of 
Rab33B on LC3 lipidation experiments will be repeated with cells transfected with either Rab33B or Atg16L1 mutants alone.

To fully understand the physiological role of the Rab33B-Atg16L1 complex the role of regulatory factors of Rab33B like GAPs and GEFs needs to be considered. The Rab-GAP OATL1 was identified to inactivate Rab33B [55]. OATL1 is localized to autophagosomal membranes by direct interactions with LC3, GABARAP and GATE16. Overexpression of OATL1 delays autophagosome maturation and fusion with the lysosome. Furthermore Rab33B and its regulator OATL1 are involved in the delivery of nano particles to autophagyrelated membranes [104]. Overexpression of Rab33B increases LC3-II levels [55]. Similar to overexpression of OATL1 increased LC3-II levels slow down autophagosome maturation and fusion with the lysosome [54]. Taken together, OATL1 could hypothetically regulate Atg12 Atg5/Atg16L1 recruitment to the pre-autophagosomal membrane by inactivating Rab33B when enough LC3 is lipidated and thereby favor maturation of the autophagosome.

For some mammalian Rab GTPases involved in autophagy orthologues have been found in yeast but no Rab33 counterpart was detected in the S. cerevisiae genome [150]. However, mammalian WIPI2B directly interacts with Atg16L1 [28]. WIPI2B is an orthologue of the yeast core autophagy protein Atg18 [108]. Very similar to yeast Atg21, it binds to PI(3)P at the phagophore, scaffolds the Atg5 Atg12/Atg16L1 complex and brings LC3 close to the membrane promoting LC3 lipidation. Atg21-Atg16 and WIPI2B-Atg16L1 binding sites are not conserved. The WIPI2B binding site of Atg16L1 includes residues 207 to 230 and is located at the extended C-terminus that is not conserved in yeast. The WIPI2B binding site of Atg16L1 is very close to but not identical with the Rab33B binding site (191-208). Preliminary co-immunoprecipitation experiments revealed that WIPI2 co-precipitates with Atg16L1 as well, as shown with a WIPI2 specific antibody (Fig. 4.2.) but findings must be verified. Still, results suspect that Rab33B and WIPI2B can simultaneously bind to Atg16. The Atg12-Atg5/Atg16L1 complex is localized at the mammalian isolation membrane and mediates LC3 lipidation. Atg16L1 may additionally recruit vesicles originating from the Golgi by simultaneously interacting with Rab33B and thus bringing these vesicles in close proximity to the isolation membrane so that they can tether and then fuse with the help of additional proteins. 

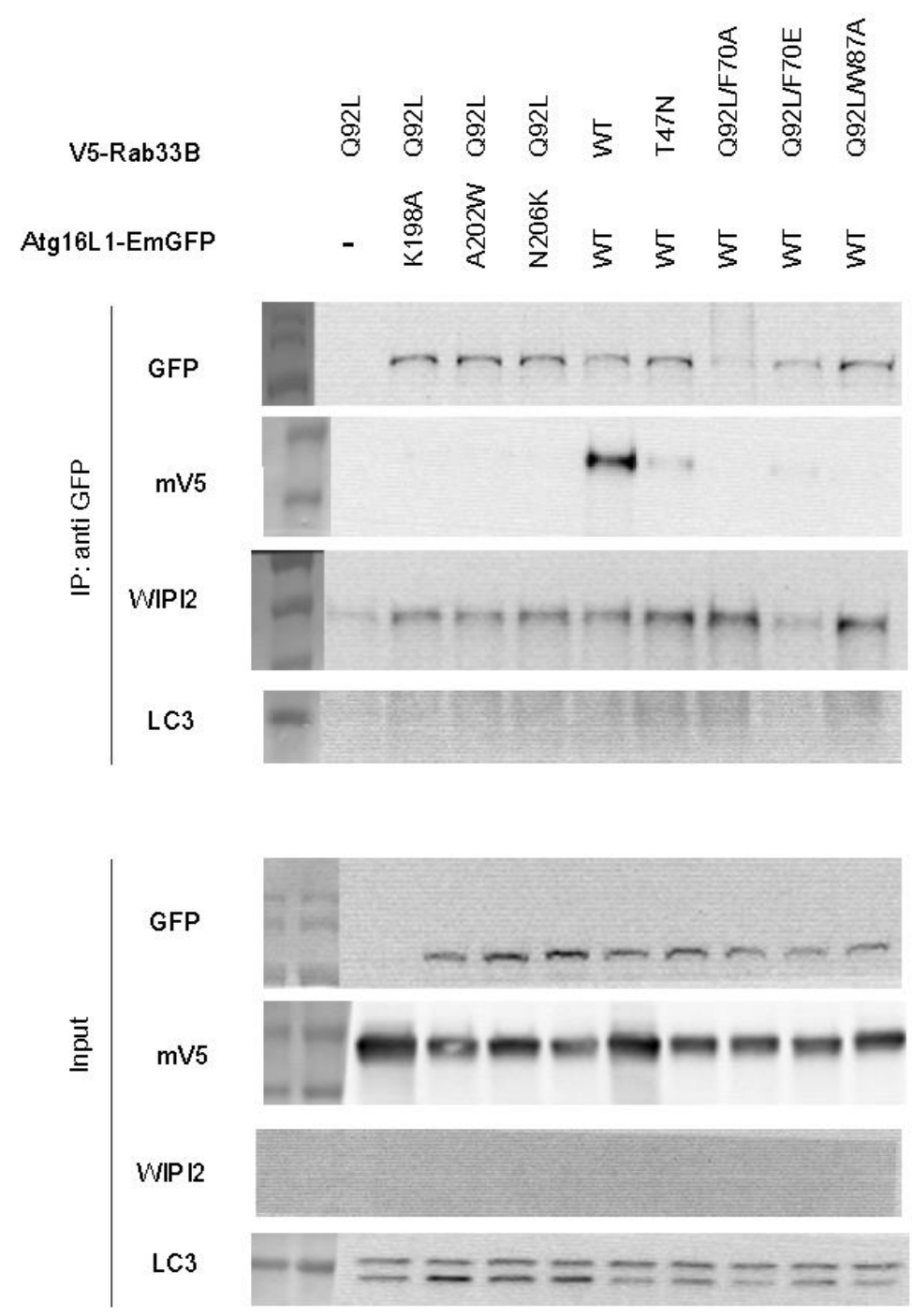

Figure 4.2.: GFP co-immunoprecipitation of Atg16L1-EmGFP and V5-Rab33B variants. Overexpression was done in HEK cells. Western blots were probed with either anti-GFP, anti-V5 antibodies or antibody specific for WIPI2B.

Rab33B is not the only factor that affects Atg16L1. Autophagy is a precisely orchestrated process that involves multiple localization factors. Recent studies propose a direct link between Atg16L1 and FIP200, a member of the autophagy initiation Atg1 complex [41]. 


\subsection{The Atg16 dimer forms the center of the Atg21-Atg16 complex}

The Atg12 Atg5/Atg16 complex is part of the core autophagy machinery. The complex acts as an E3-like ligase for Atg8 lipidation. Atg21 functions in selective subtypes of autophagy and was recently discovered to recruit both the Atg12 Atg5/Atg16 complex and Atg8 via $\mathrm{PI}(3) \mathrm{P}$ binding to the phagophore. In this way Atg12 Atg5/Atg16 and Atg3 are able to lipidate Atg8. Structural characterization of the Atg21-Atg16 complex gives more insights into the coordination of Atg8 lipidation.

In this study the minimal binding site of AgAtg16 for Atg21 interaction was identified and compromises residues 70 to 124 . The structure of the AgAtg16 coiled coil domain alone was determined at $3.4 \AA$ resolution and revealed a left-handed parallel homodimeric coiled coil similar to the previously reported ScAtg16 coiled coil domain. The AgAtg16 coiled coil dimer is mainly negatively charged at the surface and hydrophobic at the dimer interface.

SEC-MALLS measurements of the KlAtg21-AgAtg16(70-124) complex gave a molar weight of $67 \mathrm{kDa}$. The measured value for the AgAtg16 dimer is $15.4 \mathrm{kDa}$ and for KlAtg21 43.1 $\mathrm{kDa}$. This suggests that one Atg21 molecule binds one Atg16 dimer. However, this does not fit to the observations from the crystal structure where two K1Atg21 molecules and one Atg16 dimer form a complex. High protein concentration during crystallization probably favored formation of the 2:2 complex. It is very likely that the complex of two $\operatorname{Atg} 21$ molecules and one Atg16 dimer also forms in vivo because of the high local concentration of the membrane bound PROPPIN.

In this study, the structure of the KlAtg21-AgAtg16 complex was determined at $4.0 \AA$ resolution and the Atg16 binding site of Atg21 was identified. K1Atg21 forms a seven bladed $\beta$-propeller like KlHsv2, KmHsv2 and PaAtg18. The structure was determined by molecular replacement using the structures of PaAtg18 and KmHsv2 as a search model. K1Atg21AgAtg16 crystallized upon in situ proteolysis with clostripain that most probably digested all flexible loops, therefore flexible loops of the search model were truncated as well. At this low resolution most side chains cannot be built and because no SeMet crystals were obtained no sequence assignment could be done. Still the complex structure was determined with great confidence because both search models gave solutions where the propellers adopted the same orientation.

Furthermore, the known PROPPIN structures are highly conserved, only the C-terminal $\beta$ strand seven differs. In blades one to three and five to six even the connecting loops are conserved. Especially, the phosphoinositide binding sites with the FRRG motif are conserved. 
PaAtg18 was used as a model for KlAtg21 because of the stronger conservation and its better molecular replacement values. Two KlAtg21 molecules bound to the C-terminus of an AgAtg16 coiled coil dimer. The AgAtg16 binding site is located between KlAtg21 blade two and three, similar to Atg2 binding to Atg18 loops 2AB and 2BC [143]. Binding of Atg8 to Atg21 was reported opposite of the Atg16 binding site [58].

Several molecular replacement calculations were done to place the Atg16 structure using the dimer structures of ScAtg16 (PDB code 3A7O or 3A7P) and AgAtg16 with or without side chains as search models. Due to the low resolution only a few side chains of the Atg16 dimer are visible and the sequence cannot be built in with confidence for this reason. Therefore, the vertical localization of the coiled coil dimer is unknown. Coiled coil proteins are built of a repeating heptad pattern and it is possible that the coiled coil is shifted seven positions amino acid up or down. The majority of calculations placed the Atg16 dimer in a way that residues ScAtg16 90 to 108 or corresponding residues AgAtg16 67 to 85 are in close contact to Atg21. The final model was built of two KlAtg21 molecules based on the structure of PaAtg18 with one AgAtg16 dimer based on the truncated structure of AgAtg16 placed in the most frequently observed orientation. In this model a close contact for KlAtg21 R103 and AgAtg16 D78 was observed. Structural characterization of the ScAtg16 coiled coil domain revealed the significance of residues E102 and D101 which corresponds to AgAtg16 D78. Mutations of these residues to alanines affected autophagy and the Cvt pathway [37]. Another recent study showed that these residues directly interact with Atg21 [58]. I introduced charge reversing mutations K1Atg21 R103E and AgAtg16 (70-124) D78R to test complex formation by analytic gel filtration in vitro. Complex formation was disrupted for KlAtg21 WT with AgAtg16 (70-124) D78R and KlAtg21 R103E with AgAtg16 WT. But complex formation was not restored for Atg16 (70-124) D78R with KlAtg21 R103E in vitro. The interruption of complex formation for both point mutations with the corresponding wild type partner supports the importance of residues K1Atg21 R103 and AgAtg16 D78 for complex formation.

The overall structure of the KlAtg21-AgAtg16 complex shows that the bottoms of the two Atg21 propellers are facing to one side of the Atg16 dimer respectively in a reversed V shape. In contrast, the Atg21-Atg8 interaction site is on the top site of the propeller [58]. The FRRG motif with the two PIP binding sites is located opposite to the Atg16 binding site at blade five of the $\beta$-propeller. For both KlHsv2 and AgAtg18 a perpendicular or slightly tilted orientation toward the membrane was proposed $[19,69]$. 
Taking these findings together membrane binding of the Atg21-Atg16 complex suggests a Ushaped membrane around the complex. This finding is in contrast to in vivo observations that $\operatorname{Atg} 12 \sim \operatorname{Atg} 5 / \operatorname{Atg} 16$ is restricted to the outer side of the phagophore that is bent the other way round $[63,93,133]$. On the other hand Atg21 binds to PI(3)P that is evenly distributed at the inner and outer side of the membrane [100]. The two ubiquitin-like systems are tightly coupled. To favor Atg8 lipidation at the early stage of phagophore elongation Atg21 possibly localizes the Atg12 Atg5/Atg16 complex to the inner side of the cup-shaped growing isolation membrane. The Atg21-Atg16 complex is most probably formed before binding to the membrane as observed for the Atg18-Atg2 complex [101]. Furthermore, Atg16 homodimerization is independent of Atg5 and Atg12 [91]. This would suggest a mechanism where at the first step homo-oligomerized Atg21-Atg16 complex binds to the membrane and recruits the Atg5 Atg12 conjugate. Atg21 binds Atg8 and thereby brings it close to the membrane, where it is positioned for lipidation. $\operatorname{Atg} 8-\operatorname{Atg} 21$ binding is independent on the Atg5 Atg12/Atg16 complex but stabilized by Atg16 [58]. Both Atg8 and Atg5 Atg12/Atg16 are recruited to the membrane last [133]. Atg21 and the Atg12 Atg5/Atg16 probably bind to both sides of the phagophore. The distribution of the Atg21-Atg16 complex at autophagosomal membranes need to be further investigated for example by more precise microscopy techniques.

Of notice this study gives structural evidence to the model proposed earlier by Michael Thumm and Roswitha Krick (Fig. 4.3.) [72], where Atg21 binds via PI(3)P to the phagophore and recruits the Atg12 Atg5/Atg16 complex and Atg8 to the membrane in a distinct position. In this way Atg12 Atg5/Atg16 and Atg3 are localized to the lipidation site of Atg8 and act as an E3-like enzyme. Different to the model proposed Atg21 binds to negative curved membranes as found at the edge or inner part of the bend phagophore. 


\section{A}

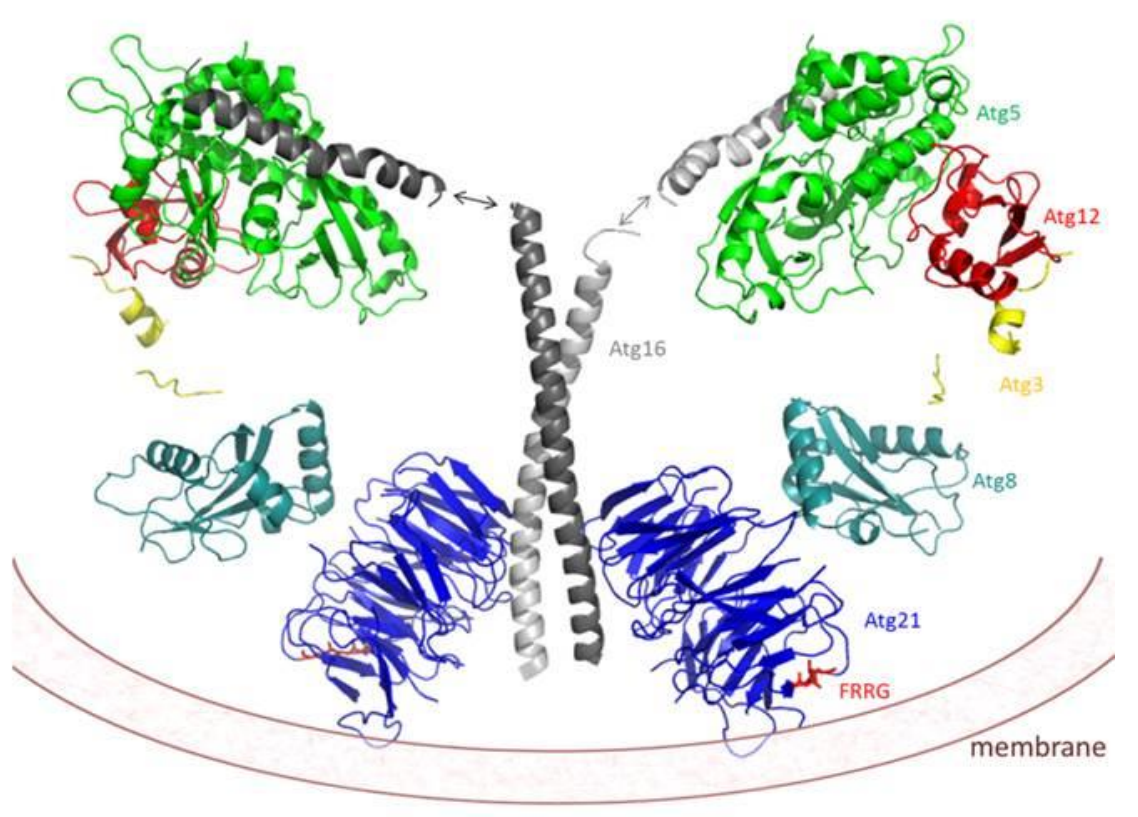

B

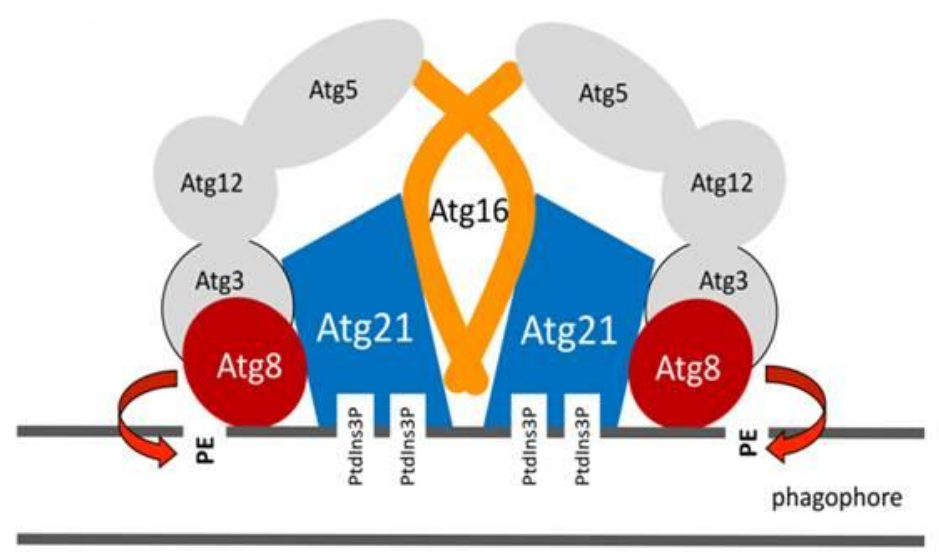

Figure 4.3.: Hypothetical model of the assembly of the autophagy ubiquitin-like conjugation system at the membrane. A: Model by superimposition of the PROPPIN-AgAtg16 model and structures from the PDB. B: Model proposed by Thumm \& Krick [72] 


\subsection{Insights into SCOC-FEZ1 complex formation}

The human SCOC-FEZ1 complex plays a role in neuronal transport processes and is involved in the induction and regulation of autophagy [86]. SCOC-FEZ1 complex formation is conserved. Mutation or deletion of the SCOC and FEZ1 orthologues in C. elegans led to a phenotype with defects in axonal outgrowth and presynaptic organization [132]. Complex formation is mediated through the highly conserved coiled coil domains of both proteins. The minimal complex SCOC(78-159)-FEZ1(227-290) was determined before [11].

In this study, I showed that the positively charged amino acids at the C-terminal part of the FEZ1 coiled coil domain stabilized complex formation with SCOC. An optimized purification protocol for the complex was developed that removes SCOC and FEZ1 that did not interact. CD measurements showed that the SCOC(78-159)-FEZ(225-295) complex adopts an overall $\alpha$-helical secondary structure as expected for coiled coil proteins that is stable with a melting temperature of $48{ }^{\circ} \mathrm{C}$. The molecular weight of the complex (44 kDa) was determined with SEC-MALLS measurements, which corresponds to a complex composed of one SCOC and one FEZ1 dimer. This result confirms the findings of McKnight et al. [86] who reported a 2:2 stoichiometric complex of about $300 \mathrm{kDa}$ complex for full length FEZ1-GFP in complex with full length FLAG-SCOC and the observation that SCOC dimerization is crucial for complex formation with FEZ1 [11].

My aim was to solve the structure of the SCOC-FEZ1 complex and while this was not achieved I still gained important new insights into the complex architecture. Although native and selenomethionine labeled crystals were obtained, which diffracted up to $2.2 \AA$ resolution all attempts to solve the structure from these crystals were not successful due to a twinning problem. However, cross-linking of the complex and analysis by mass spectroscopy gave new insights into SCOC-FEZ1 complex formation. Results strongly suggest that the FEZ1 dimer has a parallel orientation and that it interacts with the parallel coiled coil SCOC in a parallel orientation. In this model the C-terminal part of the FEZ1 coiled coil domain (264-293) interacts with the C-terminus of SCOC (122-155). To prove this model mutagenesis studies could be done but that was not possible here due to time limitations.

The importance of the SCOC C-terminus for complex formation could be studied by binding experiments with a C-terminal truncated SCOC construct. Although an interaction of SCOC with the FEZ1 homolog FEZ2 was found in a yeast-two-hybrid screen [3], no direct SCOCFEZ2 complex formation has been demonstrated so far. FEZ1 and FEZ2 share a conserved coiled coil domain but only little is known about FEZ2 until now [3]. Autophagy is also 
regulated by the interaction of the SCOC-FEZ1 complex with ULK1 and UVRAG, components of the Beclin-1 complex [86]. Further investigations on the trimeric SCOCFEZ1-UVRAG complex and interaction on SCOC-FEZ1 with ULK1 would help to understand how autophagy initiation is regulated. SCOC and FEZ1 also interact with a diverse range of proteins. Almost all FEZ1 interaction partners share a coiled coil domain as common binding motif [6]. Structural characterization of the FEZ1 coiled coil domain alone or in complex with another interaction partner would be of great interest because it might help to understand how FEZ1 can be involved in diverse interactions and fulfill various functional roles. 


\section{Bibliography}

1. Adams, P.D., Afonine, P.V., Bunkoczi, G., Chen, V.B., Davis, I.W., Echols, N., Headd, J.J., Hung, L.W., Kapral, G.J., Grosse-Kunstleve, R.W., McCoy, A.J., Moriarty, N.W., Oeffner, R., Read, R.J., Richardson, D.C., Richardson, J.S., Terwilliger, T.C., and Zwart, P.H., PHENIX: a comprehensive Python-based system for macromolecular structure solution. Acta Crystallogr D Biol Crystallogr, 2010. 66(Pt 2): p. 213-21.

2. Afonine, P.V., Grosse-Kunstleve, R.W., Echols, N., Headd, J.J., Moriarty, N.W., Mustyakimov, M., Terwilliger, T.C., Urzhumtsev, A., Zwart, P.H., and Adams, P.D., Towards automated crystallographic structure refinement with phenix.refine. Acta Crystallographica Section D, 2012. 68(4): p. 352-367.

3. Alborghetti, M.R., Furlan, A.S., and Kobarg, J., FEZ2 has acquired additional protein interaction partners relative to FEZ1: functional and evolutionary implications. PLoS One, 2011. 6(3): p. e17426.

4. Ao, X., Zou, L., and Wu, Y., Regulation of autophagy by the Rab GTPase network. Cell Death Differ, 2014. 21(3): p. 348-58.

5. Armougom, F., Moretti, S., Poirot, O., Audic, S., Dumas, P., Schaeli, B., Keduas, V., and Notredame, C., Expresso: automatic incorporation of structural information in multiple sequence alignments using 3D-Coffee. Nucleic Acids Res, 2006. 34(Web Server issue): p. W604-8.

6. Assmann, E.M., Alborghetti, M.R., Camargo, M.E., and Kobarg, J., FEZ1 dimerization and interaction with transcription regulatory proteins involves its coiled-coil region. J Biol Chem, 2006. 281(15): p. 9869-81.

7. Baba, M., Takeshige, K., Baba, N., and Ohsumi, Y., Ultrastructural analysis of the autophagic process in yeast: detection of autophagosomes and their characterization. J Cell Biol, 1994. 124(6): p. 903-13.

8. Balla, T., Phosphoinositides: tiny lipids with giant impact on cell regulation. Physiol Rev, 2013. 93(3): p. 1019-137.

9. Baskaran, S., Ragusa, M.J., Boura, E., and Hurley, J.H., Two-site recognition of phosphatidylinositol 3-phosphate by PROPPINs in autophagy. Mol Cell, 2012. 47(3): p. 33948.

10. Beck, T., Krasauskas, A., Gruene, T., and Sheldrick, G.M., A magic triangle for experimental phasing of macromolecules. Acta Crystallographica Section D, 2008. 64(11): p. 1179-1182.

11. Behrens, C., Binotti, B., Schmidt, C., Robinson, C.V., Chua, J.J., and Kühnel, K., Crystal structure of the human short coiled coil protein and insights into SCOC-FEZ1 complex formation. PLoS One, 2013. 8(10): p. e76355.

12. Behrens, C.A.J. Crystal Structure and Characterization of the SCOC Coiled Coil Domain. Doctoral thesis 2014; Available from: http://hdl.handle.net/11858/00-1735-0000-0022-5F44F.

13. Bento, C.F., Renna, M., Ghislat, G., Puri, C., Ashkenazi, A., Vicinanza, M., Menzies, F.M., and Rubinsztein, D.C., Mammalian Autophagy: How Does It Work? Annu Rev Biochem, 2016. 85: p. 685-713.

14. Binotti, B., Pavlos, N.J., Riedel, D., Wenzel, D., Vorbruggen, G., Schalk, A.M., Kühnel, K., Boyken, J., Erck, C., Martens, H., Chua, J.J., and Jahn, R., The GTPase Rab26 links synaptic vesicles to the autophagy pathway. Elife, 2015. 4: p. e05597.

15. Bloom, L. and Horvitz, H.R., The Caenorhabditis elegans gene unc-76 and its human homologs define a new gene family involved in axonal outgrowth and fasciculation. Proc Natl Acad Sci U S A, 1997. 94(7): p. 3414-9.

16. Bowler, M.W., Mueller, U., Weiss, M.S., Sanchez-Weatherby, J., Sorensen, T.L., Thunnissen, M.M., Ursby, T., Gobbo, A., Russi, S., and Bowler, M.G., Automation and experience of controlled crystal dehydration: Results from the european synchrotron hc1 collaboration. Crystal Growth \& Design, 2015. 15(3): p. 1043-1054.

17. Bradford, M.M., A rapid and sensitive method for the quantitation of microgram quantities of protein utilizing the principle of protein-dye binding. Anal Biochem, 1976. 72: p. 248-54. 
18. Burman, C. and Ktistakis, N.T., Regulation of autophagy by phosphatidylinositol 3-phosphate. FEBS Lett, 2010. 584(7): p. 1302-12.

19. Busse, R.A., Scacioc, A., Krick, R., Pérez-Lara, Á., Thumm, M., and Kühnel, K., Characterization of PROPPIN-Phosphoinositide Binding and Role of Loop 6CD in PROPPINMembrane Binding. Biophys J, 2015. 108(9): p. 2223-34.

20. Butkevich, E., Hartig, W., Nikolov, M., Erck, C., Grosche, J., Urlaub, H., Schmidt, C.F., Klopfenstein, D.R., and Chua, J.J., Phosphorylation of FEZ1 by Microtubule Affinity Regulating Kinases regulates its function in presynaptic protein trafficking. Sci Rep, 2016. 6: p. 26965.

21. Chandra, M., Saran, R., and Datta, S., Deciphering the role of Atg5 in nucleotide dependent interaction of Rab33B with the dimeric complex, Atg5-Atg16L1. Biochemical and biophysical research communications, 2016. 473(1): p. 8-16.

22. Chari, A., Haselbach, D., Kirves, J.-M., Ohmer, J., Paknia, E., Fischer, N., Ganichkin, O., Moller, V., Frye, J.J., Petzold, G., Jarvis, M., Tietzel, M., Grimm, C., Peters, J.-M., Schulman, B.A., Tittmann, K., Markl, J., Fischer, U., and Stark, H., ProteoPlex: stability optimization of macromolecular complexes by sparse-matrix screening of chemical space. Nat Meth, 2015. 12(9): p. 859-865.

23. Chari, A., Haselbach, D., Kirves, J.M., Ohmer, J., Paknia, E., Fischer, N., Ganichkin, O., Moller, V., Frye, J.J., Petzold, G., Jarvis, M., Tietzel, M., Grimm, C., Peters, J.M., Schulman, B.A., Tittmann, K., Markl, J., Fischer, U., and Stark, H., ProteoPlex: stability optimization of macromolecular complexes by sparse-matrix screening of chemical space. Nat Methods, 2015. 12(9): p. 859-65.

24. Chua, C.E., Gan, B.Q., and Tang, B.L., Involvement of members of the Rab family and related small GTPases in autophagosome formation and maturation. Cell Mol Life Sci, 2011. 68(20): p. 3349-58.

25. Chua, J.J., Butkevich, E., Worseck, J.M., Kittelmann, M., Gronborg, M., Behrmann, E., Stelzl, U., Pavlos, N.J., Lalowski, M.M., Eimer, S., Wanker, E.E., Klopfenstein, D.R., and Jahn, R., Phosphorylation-regulated axonal dependent transport of syntaxin 1 is mediated by a Kinesin1 adapter. Proc Natl Acad Sci U S A, 2012. 109(15): p. 5862-7.

26. Combe, C.W., Fischer, L., and Rappsilber, J., xiNET: cross-link network maps with residue resolution. Mol Cell Proteomics, 2015. 14(4): p. 1137-47.

27. Delano, W.L. Pymol: An open-source molecular graphics tool. 2002; Available from: http://www.pymol.org.

28. Dooley, H.C., Razi, M., Polson, H.E., Girardin, S.E., Wilson, M.I., and Tooze, S.A., WIPI2 links LC3 conjugation with PI3P, autophagosome formation, and pathogen clearance by recruiting Atg12-5-16L1. Molecular cell, 2014. 55(2): p. 238-252.

29. Dove, S.K., Piper, R.C., McEwen, R.K., Yu, J.W., King, M.C., Hughes, D.C., Thuring, J., Holmes, A.B., Cooke, F.T., Michell, R.H., Parker, P.J., and Lemmon, M.A., Svp1p defines a family of phosphatidylinositol 3,5-bisphosphate effectors. EMBO J, 2004. 23(9): p. 1922-33.

30. Döring, T. and Prange, R., Rab33B and its autophagic Atg5/12/16L1 effector assist in hepatitis B virus naked capsid formation and release. Cellular microbiology, 2015. 17(5): p. 747-764.

31. Eathiraj, S., Pan, X., Ritacco, C., and Lambright, D.G., Structural basis of family-wide Rab GTPase recognition by rabenosyn-5. Nature, 2005. 436(7049): p. 415-9.

32. Efe, J.A., Botelho, R.J., and Emr, S.D., Atg18 regulates organelle morphology and Fab1 kinase activity independent of its membrane recruitment by phosphatidylinositol 3, 5bisphosphate. Molecular biology of the cell, 2007. 18(11): p. 4232-4244.

33. Emsley, P. and Cowtan, K., Coot: model-building tools for molecular graphics. Acta Crystallogr D Biol Crystallogr, 2004. 60(Pt 12 Pt 1): p. 2126-32.

34. Ericsson, U.B., Hallberg, B.M., Detitta, G.T., Dekker, N., and Nordlund, P., Thermofluorbased high-throughput stability optimization of proteins for structural studies. Anal Biochem, 2006. 357(2): p. 289-98.

35. Farre, J.C., Krick, R., Subramani, S., and Thumm, M., Turnover of organelles by autophagy in yeast. Curr Opin Cell Biol, 2009. 21(4): p. 522-30. 
36. Fujioka, Y., Noda, N.N., Fujii, K., Yoshimoto, K., Ohsumi, Y., and Inagaki, F., In vitro reconstitution of plant Atg8 and Atg12 conjugation systems essential for autophagy. J Biol Chem, 2008. 283(4): p. 1921-8.

37. Fujioka, Y., Noda, N.N., Nakatogawa, H., Ohsumi, Y., and Inagaki, F., Dimeric coiled-coil structure of Saccharomyces cerevisiae Atg16 and its functional significance in autophagy. Journal of Biological Chemistry, 2010. 285(2): p. 1508-1515.

38. Fujita, N. and Yoshimori, T., Ubiquitination-mediated autophagy against invading bacteria. Current opinion in cell biology, 2011. 23(4): p. 492-497.

39. Fujita, T., Ikuta, J., Hamada, J., Okajima, T., Tatematsu, K., Tanizawa, K., and Kuroda, S., Identification of a tissue-non-specific homologue of axonal fasciculation and elongation protein zeta-1. Biochem Biophys Res Commun, 2004. 313(3): p. 738-44.

40. Fukuda, M. and Itoh, T., Direct link between Atg protein and small GTPase Rab: Atg16L functions as a potential Rab33 effector in mammals. Autophagy, 2008. 4(6): p. 824-826.

41. Gammoh, N., Florey, O., Overholtzer, M., and Jiang, X., Interaction between FIP200 and ATG16L1 distinguishes ULK1 complex-dependent and -independent autophagy. Nat Struct Mol Biol, 2013. 20(2): p. 144-9.

42. Geng, J. and Klionsky, D.J., The Atg8 and Atg12 ubiquitin-like conjugation systems in macroautophagy. EMBO reports, 2008. 9(9): p. 859-864.

43. Geng, J. and Klionsky, D.J., The Golgi as a potential membrane source for autophagy. Autophagy, 2010. 6(7): p. 950-1.

44. Glaser, F., Pupko, T., Paz, I., Bell, R.E., Bechor-Shental, D., Martz, E., and Ben-Tal, N., ConSurf: identification of functional regions in proteins by surface-mapping of phylogenetic information. Bioinformatics, 2003. 19(1): p. 163-164.

45. Goitre, L., Trapani, E., Trabalzini, L., and Retta, S.F., The Ras superfamily of small GTPases: the unlocked secrets. Methods Mol Biol, 2014. 1120: p. 1-18.

46. Grigoryan, G. and Keating, A.E., Structural specificity in coiled-coil interactions. Curr Opin Struct Biol, 2008. 18(4): p. 477-83.

47. Hailey, D.W., Rambold, A.S., Satpute-Krishnan, P., Mitra, K., Sougrat, R., Kim, P.K., and Lippincott-Schwartz, J., Mitochondria supply membranes for autophagosome biogenesis during starvation. Cell, 2010. 141(4): p. 656-67.

48. Hain, A.U., Weltzer, R.R., Hammond, H., Jayabalasingham, B., Dinglasan, R.R., Graham, D.R., Colquhoun, D.R., Coppens, I., and Bosch, J., Structural characterization and inhibition of the Plasmodium Atg8-Atg3 interaction. J Struct Biol, 2012. 180(3): p. 551-62.

49. Hanada, T., Noda, N.N., Satomi, Y., Ichimura, Y., Fujioka, Y., Takao, T., Inagaki, F., and Ohsumi, Y., The Atg12-Atg5 conjugate has a novel E3-like activity for protein lipidation in autophagy. J Biol Chem, 2007. 282(52): p. 37298-302.

50. Harbury, P.B., Zhang, T., Kim, P.S., and Alber, T., A Switch Between Two-, Three-, and Four-Stranded Coiled Coils in GCN4 Leucine Zipper Mutants. Science, 1993. 262: p. 26.

51. Harding, T.M., Morano, K.A., Scott, S.V., and Klionsky, D.J., Isolation and characterization of yeast mutants in the cytoplasm to vacuole protein targeting pathway. The Journal of cell biology, 1995. 131(3): p. 591-602.

52. Heras, B. and Martin, J.L., Post-crystallization treatments for improving diffraction quality of protein crystals. Acta Crystallographica Section D, 2005. 61(9): p. 1173-1180.

53. Ishibashi, K., Fujita, N., Kanno, E., Omori, H., Yoshimori, T., Itoh, T., and Fukuda, M., Atg16L2, a novel isoform of mammalian Atg16 that is not essential for canonical autophagy despite forming an Atg12-5-16L2 complex. Autophagy, 2011. 7(12): p. 1500-1513.

54. Itoh, T., Fujita, N., Kanno, E., Yamamoto, A., Yoshimori, T., and Fukuda, M., Golgi-resident small GTPase Rab33B interacts with Atg16L and modulates autophagosome formation. Mol Biol Cell, 2008. 19(7): p. 2916-25.

55. Itoh, T., Kanno, E., Uemura, T., Waguri, S., and Fukuda, M., OATL1, a novel autophagosome-resident Rab33B-GAP, regulates autophagosomal maturation. J Cell Biol, 2011. 192(5): p. 839-53.

56. Jenny, R.J., Mann, K.G., and Lundblad, R.L., A critical review of the methods for cleavage of fusion proteins with thrombin and factor Xa. Protein Expr Purif, 2003. 31(1): p. 1-11.

57. Joachim, J., Wirth, M., McKnight, N.C., and Tooze, S.A., Coiling up with SCOC and WAC: two new regulators of starvation-induced autophagy. Autophagy, 2012. 8(9): p. 1397-400. 
58. Juris, L., Montino, M., Rube, P., Schlotterhose, P., Thumm, M., and Krick, R., PI3P binding by Atg21 organises Atg8 lipidation. The EMBO journal, 2015. 34(7): p. 955-973.

59. Kabsch, W., Integration, scaling, space-group assignment and post-refinement. Acta Crystallogr D Biol Crystallogr, 2010. 66(Pt 2): p. 133-44.

60. Kamada, Y., Yoshino, K., Kondo, C., Kawamata, T., Oshiro, N., Yonezawa, K., and Ohsumi, Y., Tor directly controls the Atg1 kinase complex to regulate autophagy. Mol Cell Biol, 2010. 30(4): p. 1049-58.

61. Kaufmann, A., Beier, V., Franquelim, H.G., and Wollert, T., Molecular mechanism of autophagic membrane-scaffold assembly and disassembly. Cell, 2014. 156(3): p. 469-81.

62. Kaushik, S. and Cuervo, A.M., Chaperone-mediated autophagy: a unique way to enter the lysosome world. Trends Cell Biol, 2012. 22(8): p. 407-17.

63. Kirisako, T., Ichimura, Y., Okada, H., Kabeya, Y., Mizushima, N., Yoshimori, T., Ohsumi, M., Takao, T., Noda, T., and Ohsumi, Y., The reversible modification regulates the membrane-binding state of Apg8/Aut7 essential for autophagy and the cytoplasm to vacuole targeting pathway. J Cell Biol, 2000. 151(2): p. 263-76.

64. Kirkin, V., McEwan, D.G., Novak, I., and Dikic, I., A role for ubiquitin in selective autophagy. Molecular cell, 2009. 34(3): p. 259-269.

65. Klionsky, D.J. and Emr, S.D., Autophagy as a regulated pathway of cellular degradation. Science, 2000. 290(5497): p. 1717-1721.

66. Knodler, L.A. and Celli, J., Eating the strangers within: host control of intracellular bacteria via xenophagy. Cell Microbiol, 2011. 13(9): p. 1319-27.

67. Kondo-Okamoto, N., Noda, N.N., Suzuki, S.W., Nakatogawa, H., Takahashi, I., Matsunami, M., Hashimoto, A., Inagaki, F., Ohsumi, Y., and Okamoto, K., Autophagy-related protein 32 acts as autophagic degron and directly initiates mitophagy. J Biol Chem, 2012. 287(13): p. 10631-8.

68. Kraft, C. and Martens, S., Mechanisms and regulation of autophagosome formation. Curr Opin Cell Biol, 2012. 24(4): p. 496-501.

69. Krick, R., Busse, R.A., Scacioc, A., Stephan, M., Janshoff, A., Thumm, M., and Kühnel, K., Structural and functional characterization of the two phosphoinositide binding sites of PROPPINs, a beta-propeller protein family. Proc Natl Acad Sci U S A, 2012. 109(30): p. E2042-9.

70. Krick, R., Henke, S., Tolstrup, J., and Thumm, M., Dissecting the localization and function of Atg18, Atg21 and Ygr223c. Autophagy, 2008. 4(7): p. 896-910.

71. Krick, R., Muehe, Y., Prick, T., Bremer, S., Schlotterhose, P., Eskelinen, E.L., Millen, J., Goldfarb, D.S., and Thumm, M., Piecemeal microautophagy of the nucleus requires the core macroautophagy genes. Mol Biol Cell, 2008. 19(10): p. 4492-505.

72. Krick, R. and Thumm, M., Atg8 lipidation is coordinated in a PtdIns3P-dependent manner by the PROPPIN Atg21. Autophagy, 2016. 12(11): p. 2260-2261.

73. Krick, R., Tolstrup, J., Appelles, A., Henke, S., and Thumm, M., The relevance of the phosphatidylinositolphosphat-binding motif FRRGT of Atg18 and Atg21 for the Cvt pathway and autophagy. FEBS Lett, 2006. 580(19): p. 4632-8.

74. Ktistakis, N.T. and Tooze, S.A., Digesting the Expanding Mechanisms of Autophagy. Trends Cell Biol, 2016. 26(8): p. 624-35.

75. Kuma, A., Mizushima, N., Ishihara, N., and Ohsumi, Y., Formation of the approximately 350$\mathrm{kDa}$ Apg12-Apg5.Apg16 multimeric complex, mediated by Apg16 oligomerization, is essential for autophagy in yeast. J Biol Chem, 2002. 277(21): p. 18619-25.

76. Lanza, D.C., Silva, J.C., Assmann, E.M., Quaresma, A.J., Bressan, G.C., Torriani, I.L., and Kobarg, J., Human FEZ1 has characteristics of a natively unfolded protein and dimerizes in solution. Proteins, 2009. 74(1): p. 104-21.

77. Li, W.W., Li, J., and Bao, J.K., Microautophagy: lesser-known self-eating. Cell Mol Life Sci, 2012. 69(7): p. 1125-36.

78. Liu, X. and Klionsky, D.J., The Atg17-Atg31-Atg29 complex and Atg11 regulate autophagosome-vacuole fusion. Autophagy, 2016. 12(5): p. 894-5.

79. Liu, X., Mao, K., Yu, A.Y., Omairi-Nasser, A., Austin, J., 2nd, Glick, B.S., Yip, C.K., and Klionsky, D.J., The Atg17-Atg31-Atg29 Complex Coordinates with Atg11 to Recruit the 
Vam7 SNARE and Mediate Autophagosome-Vacuole Fusion. Curr Biol, 2016. 26(2): p. 15060 .

80. Lynch-Day, M.A. and Klionsky, D.J., The Cvt pathway as a model for selective autophagy. FEBS letters, 2010. 584(7): p. 1359-1366.

81. Mao, K., Chew, L.H., Inoue-Aono, Y., Cheong, H., Nair, U., Popelka, H., Yip, C.K., and Klionsky, D.J., Atg29 phosphorylation regulates coordination of the Atg17-Atg31-Atg29 complex with the Atg11 scaffold during autophagy initiation. Proc Natl Acad Sci U S A, 2013. 110(31): p. E2875-84.

82. Mari, M. and Reggiori, F., Atg9 reservoirs, a new organelle of the yeast endomembrane system? Autophagy, 2010. 6(8): p. 1221-3.

83. Matsushita, M., Suzuki, N.N., Obara, K., Fujioka, Y., Ohsumi, Y., and Inagaki, F., Structure of Atg5. Atg16, a complex essential for autophagy. Journal of Biological Chemistry, 2007. 282(9): p. 6763-6772.

84. Matthews, B.W., Solvent content of protein crystals. Journal of molecular biology, 1968. 33(2): p. 491-497.

85. McCoy, A.J., Grosse-Kunstleve, R.W., Adams, P.D., Winn, M.D., Storoni, L.C., and Read, R.J., Phaser crystallographic software. Journal of Applied Crystallography, 2007. 40(4): p. 658-674.

86. McKnight, N.C., Jefferies, H.B., Alemu, E.A., Saunders, R.E., Howell, M., Johansen, T., and Tooze, S.A., Genome-wide siRNA screen reveals amino acid starvation-induced autophagy requires SCOC and WAC. EMBO J, 2012. 31(8): p. 1931-46.

87. Merkley, E.D., Rysavy, S., Kahraman, A., Hafen, R.P., Daggett, V., and Adkins, J.N., Distance restraints from crosslinking mass spectrometry: mining a molecular dynamics simulation database to evaluate lysine-lysine distances. Protein Science, 2014. 23(6): p. 747759.

88. Milburn, M.V., Tong, L., deVos, A.M., Brunger, A., Yamaizumi, Z., Nishimura, S., and Kim, S.H., Molecular switch for signal transduction: structural differences between active and inactive forms of protooncogenic ras proteins. Science, 1990. 247(4945): p. 939-45.

89. Mizushima, N. and Komatsu, M., Autophagy: renovation of cells and tissues. Cell, 2011. 147(4): p. 728-41.

90. Mizushima, N., Kuma, A., Kobayashi, Y., Yamamoto, A., Matsubae, M., Takao, T., Natsume, T., Ohsumi, Y., and Yoshimori, T., Mouse Apg 16L, a novel WD-repeat protein, targets to the autophagic isolation membrane with the Apg12-Apg5 conjugate. J Cell Sci, 2003. 116(Pt 9): p. $1679-88$.

91. Mizushima, N., Noda, T., and Ohsumi, Y., Apg16p is required for the function of the Apg12pApg5p conjugate in the yeast autophagy pathway. EMBO J, 1999. 18(14): p. 3888-96.

92. Mizushima, N., Yoshimori, T., and Levine, B., Methods in mammalian autophagy research. Cell, 2010. 140(3): p. 313-26.

93. Mizushima, N., Yoshimori, T., and Ohsumi, Y., The role of Atg proteins in autophagosome formation. Annual review of cell and developmental biology, 2011. 27: p. 107-132.

94. Murshudov, G.N., Skubak, P., Lebedev, A.A., Pannu, N.S., Steiner, R.A., Nicholls, R.A., Winn, M.D., Long, F., and Vagin, A.A., REFMAC5 for the refinement of macromolecular crystal structures. Acta Crystallogr D Biol Crystallogr, 2011. 67(Pt 4): p. 355-67.

95. Nair, U., Yen, W.L., Mari, M., Cao, Y., Xie, Z., Baba, M., Reggiori, F., and Klionsky, D.J., A role for Atg8-PE deconjugation in autophagosome biogenesis. Autophagy, 2012. 8(5): p. 78093.

96. Nakatogawa, H., Ichimura, Y., and Ohsumi, Y., Atg8, a ubiquitin-like protein required for autophagosome formation, mediates membrane tethering and hemifusion. Cell, 2007. 130(1): p. 165-78.

97. Nakatogawa, H., Suzuki, K., Kamada, Y., and Ohsumi, Y., Dynamics and diversity in autophagy mechanisms: lessons from yeast. Nat Rev Mol Cell Biol, 2009. 10(7): p. 458-67.

98. Nishida, Y., Arakawa, S., Fujitani, K., Yamaguchi, H., Mizuta, T., Kanaseki, T., Komatsu, M., Otsu, K., Tsujimoto, Y., and Shimizu, S., Discovery of Atg5/Atg7-independent alternative macroautophagy. Nature, 2009. 461(7264): p. 654-658. 
99. Noda, N.N., Fujioka, Y., Hanada, T., Ohsumi, Y., and Inagaki, F., Structure of the Atg12Atg5 conjugate reveals a platform for stimulating Atg8-PE conjugation. EMBO Rep, 2013. 14(2): p. 206-11.

100. Obara, K. and Ohsumi, Y., Dynamics and function of PtdIns(3)P in autophagy. Autophagy, 2008. 4(7): p. 952-4.

101. Obara, K., Sekito, T., Niimi, K., and Ohsumi, Y., The Atg18-Atg2 complex is recruited to autophagic membranes via phosphatidylinositol 3-phosphate and exerts an essential function. $\mathbf{J}$ Biol Chem, 2008. 283(35): p. 23972-80.

102. Otomo, C., Metlagel, Z., Takaesu, G., and Otomo, T., Structure of the human ATG12 ATG5 conjugate required for LC3 lipidation in autophagy. Nat Struct Mol Biol, 2013. 20(1): p. 5966.

103. Pan, X., Eathiraj, S., Munson, M., and Lambright, D.G., TBC-domain GAPs for Rab GTPases accelerate GTP hydrolysis by a dual-finger mechanism. Nature, 2006. 442(7100): p. 303-6.

104. Panarella, A., Bexiga, M.G., Galea, G., ED, O.N., Salvati, A., Dawson, K.A., and Simpson, J.C., A systematic High-Content Screening microscopy approach reveals key roles for Rab33b, OATL1 and Myo6 in nanoparticle trafficking in HeLa cells. Sci Rep, 2016. 6: p. 28865.

105. Panic, B., Whyte, J.R., and Munro, S., The ARF-like GTPases Arl1p and Arl3p act in a pathway that interacts with vesicle-tethering factors at the Golgi apparatus. Curr Biol, 2003. 13(5): p. 405-10.

106. Papinski, D., Schuschnig, M., Reiter, W., Wilhelm, L., Barnes, C.A., Maiolica, A., Hansmann, I., Pfaffenwimmer, T., Kijanska, M., Stoffel, I., Lee, S.S., Brezovich, A., Lou, J.H., Turk, B.E., Aebersold, R., Ammerer, G., Peter, M., and Kraft, C., Early steps in autophagy depend on direct phosphorylation of Atg9 by the Atg1 kinase. Mol Cell, 2014. 53(3): p. 471-83.

107. Parkhouse, R., Ebong, I.-O., Robinson, C.V., and Monie, T.P., The N-terminal region of the human autophagy protein ATG16L1 contains a domain that folds into a helical structure consistent with formation of a coiled-coil. PloS one, 2013. 8(9): p. e76237.

108. Polson, H.E., de Lartigue, J., Rigden, D.J., Reedijk, M., Urbé, S., Clague, M.J., and Tooze, S.A., Mammalian Atg18 (WIPI2) localizes to omegasome-anchored phagophores and positively regulates LC3 lipidation. Autophagy, 2010. 6(4): p. 506-522.

109. Proikas-Cezanne, T. and Robenek, H., Freeze-fracture replica immunolabelling reveals human WIPI-1 and WIPI-2 as membrane proteins of autophagosomes. J Cell Mol Med, 2011. 15(9): p. 2007-10.

110. Proikas-Cezanne, T., Ruckerbauer, S., Stierhof, Y.-D., Berg, C., and Nordheim, A., Human WIPI-1 puncta-formation: a novel assay to assess mammalian autophagy. FEBS letters, 2007. 581(18): p. 3396-3404.

111. Ravikumar, B., Moreau, K., Jahreiss, L., Puri, C., and Rubinsztein, D.C., Plasma membrane contributes to the formation of pre-autophagosomal structures. Nat Cell Biol, 2010. 12(8): p. 747-57.

112. Reggiori, F., 1. Membrane origin for autophagy. Curr Top Dev Biol, 2006. 74: p. 1-30.

113. Reggiori, F. and Klionsky, D.J., Autophagic processes in yeast: mechanism, machinery and regulation. Genetics, 2013. 194(2): p. 341-361.

114. Reggiori, F. and Tooze, S.A., Autophagy regulation through Atg9 traffic. J Cell Biol, 2012. 198(2): p. 151-3.

115. Reggiori, F., Tucker, K.A., Stromhaug, P.E., and Klionsky, D.J., The Atg1-Atg13 complex regulates Atg9 and Atg23 retrieval transport from the pre-autophagosomal structure. Developmental cell, 2004. 6(1): p. 79-90.

116. Robert, X. and Gouet, P., Deciphering key features in protein structures with the new ENDscript server. Nucleic Acids Research, 2014. 42(W1): p. W320-W324.

117. Roberts, P., Moshitch-Moshkovitz, S., Kvam, E., O'Toole, E., Winey, M., and Goldfarb, D.S., Piecemeal microautophagy of nucleus in Saccharomyces cerevisiae. Molecular biology of the cell, 2003. 14(1): p. 129-141.

118. Saraste, M., Sibbald, P.R., and Wittinghofer, A., The P-loop-a common motif in ATP-and GTP-binding proteins. Trends in biochemical sciences, 1990. 15(11): p. 430-434. 
119. Scacioc, A. Structural, biochemical and computational studies on PROPPINs, proteins important in autophagy. Doctoral thesis 2015; Available from: http://hdl.handle.net/11858/001735-0000-0028-8675-3.

120. Schagger, H. and von Jagow, G., Tricine-sodium dodecyl sulfate-polyacrylamide gel electrophoresis for the separation of proteins in the range from 1 to $100 \mathrm{kDa}$. Anal Biochem, 1987. 166(2): p. 368-79.

121. Schalk, A.M. Structural and functional characterization of the autophagy proteins Atg5 and Atg16L1 and their interaction partners. Doctoral Thesis 2011; Available from: http://hdl.handle.net/11858/00-1735-0000-0006-AE0A-0.

122. Schlichting, I., Almo, S., Rapp, G., Wilson, K., Petratos, K., Lentfer, A., Wittinghofer, A., Kabsch, W., Pai, E., and Petsko, G., Time resolved X-ray structural studies on H-ras p21: Identification of the conformational change induced by GTP hydrolysis. Nature, 1990. 345: p. 309-315.

123. Schu, P.V., Takegawa, K., Fry, M.J., Stack, J.H., Waterfield, M.D., and Emr, S.D., Phosphatidylinositol 3-kinase encoded by yeast VPS34 gene essential for protein sorting. Science, 1993. 260(5104): p. 88-91.

124. Schwartz, S.L., Cao, C., Pylypenko, O., Rak, A., and Wandinger-Ness, A., Rab GTPases at a glance. J Cell Sci, 2007. 120(Pt 22): p. 3905-10.

125. Shen, H.M. and Mizushima, N., At the end of the autophagic road: an emerging understanding of lysosomal functions in autophagy. Trends Biochem Sci, 2014. 39(2): p. 61-71.

126. Smith, T.F., Diversity of WD-repeat proteins. Subcell Biochem, 2008. 48: p. 20-30.

127. Starr, T., Sun, Y., Wilkins, N., and Storrie, B., Rab33b and Rab6 are functionally overlapping regulators of Golgi homeostasis and trafficking. Traffic, 2010. 11(5): p. 626-636.

128. Stein, M.P., Cao, C., Tessema, M., Feng, Y., Romero, E., Welford, A., and Wandinger-Ness, A., Interaction and Functional Analyses of Human VPS34/p150 Phosphatidylinositol 3-Kinase Complex with Rab7. Methods in enzymology, 2005. 403: p. 628-649.

129. Stenmark, H. and Olkkonen, V.M., The Rab GTPase family. Genome Biol, 2001. 2(5): p. REVIEWS3007.

130. Strelkov, S.V. and Burkhard, P., Analysis of alpha-helical coiled coils with the program TWISTER reveals a structural mechanism for stutter compensation. J Struct Biol, 2002. 137(1-2): p. 54-64.

131. Stromhaug, P.E., Reggiori, F., Guan, J., Wang, C.W., and Klionsky, D.J., Atg21 is a phosphoinositide binding protein required for efficient lipidation and localization of Atg8 during uptake of aminopeptidase I by selective autophagy. Mol Biol Cell, 2004. 15(8): p. 3553-66.

132. Su, C.W., Tharin, S., Jin, Y., Wightman, B., Spector, M., Meili, D., Tsung, N., Rhiner, C., Bourikas, D., Stoeckli, E., Garriga, G., Horvitz, H.R., and Hengartner, M.O., The short coiledcoil domain-containing protein UNC-69 cooperates with UNC-76 to regulate axonal outgrowth and normal presynaptic organization in Caenorhabditis elegans. J Biol, 2006. 5(4): p. 9.

133. Suzuki, K., Akioka, M., Kondo-Kakuta, C., Yamamoto, H., and Ohsumi, Y., Fine mapping of autophagy-related proteins during autophagosome formation in Saccharomyces cerevisiae. J Cell Sci, 2013. 126(Pt 11): p. 2534-44.

134. Suzuki, N.N., Yoshimoto, K., Fujioka, Y., Ohsumi, Y., and Inagaki, F., The crystal structure of plant ATG12 and its biological implication in autophagy. Autophagy, 2005. 1(2): p. 119126.

135. Takeshige, K., Baba, M., Tsuboi, S., Noda, T., and Ohsumi, Y., Autophagy in yeast demonstrated with proteinase-deficient mutants and conditions for its induction. J Cell Biol, 1992. 119(2): p. 301-11.

136. Tamura, N., Oku, M., and Sakai, Y., Atg21 regulates pexophagy via its PI (3) P-binding activity in Pichia pastoris. FEMS yeast research, 2014. 14(3): p. 435-444.

137. Thumm, M., Egner, R., Koch, B., Schlumpberger, M., Straub, M., Veenhuis, M., and Wolf, D.H., Isolation of autophagocytosis mutants of Saccharomyces cerevisiae. FEBS Lett, 1994. 349(2): p. 275-80.

138. Tsukada, M. and Ohsumi, Y., Isolation and characterization of autophagy-defective mutants of Saccharomyces cerevisiae. FEBS Lett, 1993. 333(1-2): p. 169-74. 
139. Walczak, M. and Martens, S., Dissecting the role of the Atg12-Atg5-Atg16 complex during autophagosome formation. Autophagy, 2013. 9(3): p. 424-5.

140. Valsdottir, R., Hashimoto, H., Ashman, K., Koda, T., Storrie, B., and Nilsson, T., Identification of rabaptin-5, rabex-5, and GM130 as putative effectors of rab33b, a regulator of retrograde traffic between the Golgi apparatus and ER. FEBS letters, 2001. 508(2): p. 201209.

141. Van Valkenburgh, H., Shern, J.F., Sharer, J.D., Zhu, X., and Kahn, R.A., ADP-ribosylation factors (ARFs) and ARF-like 1 (ARL1) have both specific and shared effectors: characterizing ARL1-binding proteins. J Biol Chem, 2001. 276(25): p. 22826-37.

142. Wang, Y., Li, L., Hou, C., Lai, Y., Long, J., Liu, J., Zhong, Q., and Diao, J., SNAREmediated membrane fusion in autophagy. Semin Cell Dev Biol, 2016. 60: p. 97-104.

143. Watanabe, Y., Kobayashi, T., Yamamoto, H., Hoshida, H., Akada, R., Inagaki, F., Ohsumi, Y., and Noda, N.N., Structure-based analyses reveal distinct binding sites for Atg2 and phosphoinositides in Atg18. J Biol Chem, 2012. 287(38): p. 31681-90.

144. Wennerberg, K., Rossman, K.L., and Der, C.J., The Ras superfamily at a glance. J Cell Sci, 2005. 118(Pt 5): p. 843-6.

145. Wood, C.W., Bruning, M., Ibarra, A.A., Bartlett, G.J., Thomson, A.R., Sessions, R.B., Brady, R.L., and Woolfson, D.N., CCBuilder: an interactive web-based tool for building, designing and assessing coiled-coil protein assemblies. Bioinformatics, 2014. 30(21): p. 3029-35.

146. Yang, Z. and Klionsky, D.J., An overview of the molecular mechanism of autophagy, in Autophagy in infection and immunity. 2009, Springer. p. 1-32.

147. Yuan, H.X., Russell, R.C., and Guan, K.L., Regulation of PIK3C3/VPS34 complexes by MTOR in nutrient stress-induced autophagy. Autophagy, 2013. 9(12): p. 1983-95.

148. Zhang, Z., Wang, S., Shen, T., Chen, J., and Ding, J., Crystal structure of the Rab9ARUTBC2 RBD complex reveals the molecular basis for the binding specificity of Rab9A with RUTBC2. Structure, 2014. 22(10): p. 1408-1420.

149. Zheng, J.Y., Koda, T., Arimura, Y., Kishi, M., and Kakinuma, M., Structure and expression of the mouse S10 gene. Biochim Biophys Acta, 1997. 1351(1-2): p. 47-50.

150. Zheng, J.Y., Koda, T., Fujiwara, T., Kishi, M., Ikehara, Y., and Kakinuma, M., A novel Rab GTPase, Rab33B, is ubiquitously expressed and localized to the medial Golgi cisternae. J Cell Sci, 1998. 111 ( Pt 8): p. 1061-9.

151. Zhu, G., Zhai, P., Liu, J., Terzyan, S., Li, G., and Zhang, X.C., Structural basis of Rab5Rabaptin5 interaction in endocytosis. Nature structural \& molecular biology, 2004. 11(10): p. 975-983. 


\section{Acknowledgments}

First of all, I would like to thank Dr. Karin Kühnel for the opportunity to work in her group. I am truly grateful for the great supervision and constant support. Thank you for opening the world of protein crystallization and structure determination to me.

I would like to thank Prof. Dr. Reinhard Jahn for generous financial support, a bench place and the infrastructure in his department.

Furthermore, I would like to thank Prof. Dr. Michael Thumm, who I first met as a member of my thesis committee, who became a collaborator and who then welcomed me in his group for the last months of my $\mathrm{PhD}$ thesis. I am very grateful to my thesis committee member, Prof. Dr. Heike Krebber, for advice and feedback.

Special thanks go to Dr. Beyenech Binnotti, who did a great work on the in vivo experiments and who was always open for discussions. I am very thankful to Dr. Ángel Pérez-Lara for ITC measurements, ideas and discussion; Dr. Momchil Ninov for help with design and discussion of the cross-linking experiments; Brigitte Barg-Kues for technical assistance and Dr. Hans Dieter Schmitt for general support. I also thank bachelor student Johannes Groffmann. My thanks go to the staff of the crystallization facility, particularly Jürgen Wawrzinek and Ulrich Steuerwald for constant support. I am thankful to Dr. Sergei Trakhanov and especially to Dr. Piotr Neumann for sharing their countless knowledge about protein crystallography and who were always willing to help me. I would also like to express my gratitude towards the beamline staff at the SLS, Switzerland, at the DESY, Hamburg and BESSY, Berlin for excellent technical support. I would like to direct my acknowledgements to all those who have helped me with several methods; to Dr. Achim Dickmanns and Johannes Arens for the SECMALLS measurements; to Dr. David Haselbach for ProteoPlex measurements; to Dr. ChungTien Lee, and Monika Raabe from the department of Prof Dr. Henning Urlaub for mass spectrometry analysis.

I am thankful to the entire Neurobiology department at the MPI for the wonderful and friendly environment.

Last but not least I want to thank my family, especially Dr. Thorben Sprink for supporting and encouraging me any time and for a lot of fruitful discussions. 